\title{
WestVirginiaUniversity
}

THE RESEARCH REPOSITORY @ WVU

Graduate Theses, Dissertations, and Problem Reports

2001

\section{Processing and characterization of contacts on MBE-grown gallium nitride}

Carlo Requiao da Cunha

West Virginia University

Follow this and additional works at: https://researchrepository.wvu.edu/etd

\section{Recommended Citation}

da Cunha, Carlo Requiao, "Processing and characterization of contacts on MBE-grown gallium nitride" (2001). Graduate Theses, Dissertations, and Problem Reports. 1110.

https://researchrepository.wvu.edu/etd/1110

This Thesis is protected by copyright and/or related rights. It has been brought to you by the The Research Repository @ WVU with permission from the rights-holder(s). You are free to use this Thesis in any way that is permitted by the copyright and related rights legislation that applies to your use. For other uses you must obtain permission from the rights-holder(s) directly, unless additional rights are indicated by a Creative Commons license in the record and/ or on the work itself. This Thesis has been accepted for inclusion in WVU Graduate Theses, Dissertations, and Problem Reports collection by an authorized administrator of The Research Repository @ WVU. For more information, please contact researchrepository@mail.wvu.edu. 


\author{
Carlo Requiao da Cunha
}

Thesis submitted to the College of Engineering and Mineral Resources at West Virginia University in partial fulfillment of the requirements for the degree of

\author{
Master of Science \\ in \\ Electrical Engineering
}

Larry Hornak, Ph.D., Chair

Thomas Myers, Ph.D.

Kathleen Meehan, Ph.D.

Mark Jerabek, Ph.D.

Department of Computer Science and Electrical

Engineering

\title{
Morgantown, West Virginia \\ 2001
}

Keywords: gallium nitride, contacts, capacitance, electrical model, C-V, I-V

Copyright 2001 Carlo Requiao da Cunha 


\section{ABSTRACT \\ Processing and Characterization of Contacts on MBE-Grown Gallium Nitride}

\section{Carlo Requiao da Cunha}

Gallium nitride, a III-V compound semiconductor, is a good candidate for applications such as blue laser diodes and power devices due to its wide bandgap. One of the main challenges related to this compound is the growth of high quality crystals. If crystals with high level of purity are grown, the carrier concentration is therefore low, and the resulting high resistivity of the bulk imposes some difficulties for capacitancerelated measurements. This thesis deals with the problem of processing and characterization of contacts on highly resistive materials such as unintentionally doped ntype gallium nitride. The ideal electrical model of a depletion layer is analyzed, and modifications are proposed in order to set up a framework for measurements on low carrier concentration materials. A silicon wafer was processed to emulate the behavior of unintentionally doped gallium nitride and validate the developed characterization techniques. Measurements obtained from two samples of gallium nitride are included to illustrate how these studied methodologies can be used to obtain semiconductor parameters such as carrier concentration and built-in voltage. 


\section{Acknowledgments}

The author would like to acknowledge and thank his adviser Dr.Hornak for his support and dedication, Dr.Meehan for her immense help, and his parents, brother, and friends who helped throughout the development of this thesis. In addition the author would like to acknowledge Dr.Myers and his group for the Hall measurements and for providing the GaN samples of this study. 


\section{Table of Contents}

Abstract.

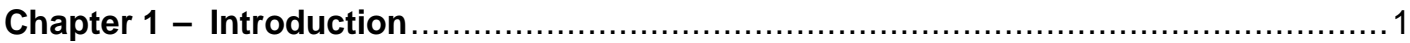

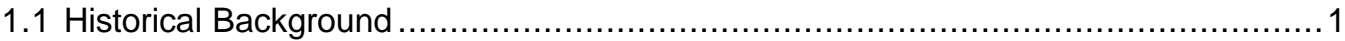

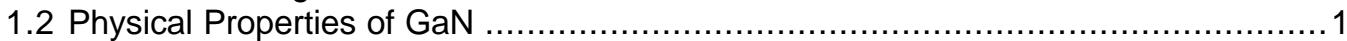

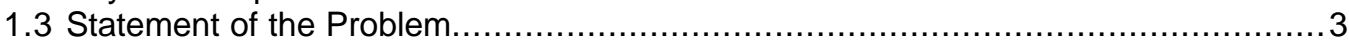

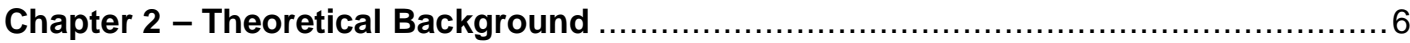

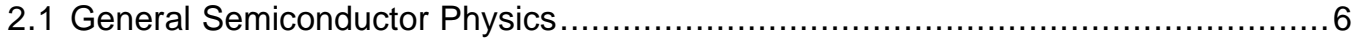

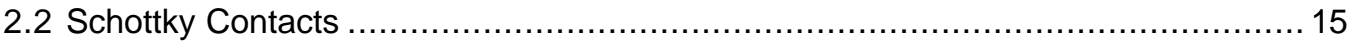

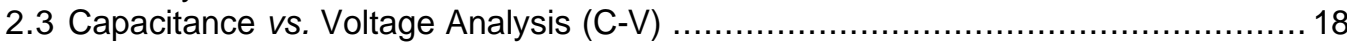

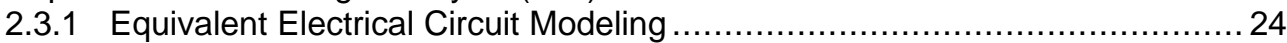

2.3.1.1 Interface Trapped Charges .................................................... 28

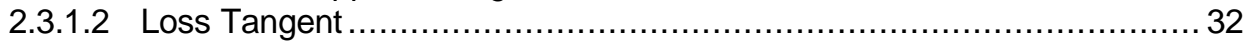

2.3.1.3 Diffusion Capacitance............................................................. 34

2.4 Current vs. Voltage Analysis (I-V) On Planar Circular Structures ......................... 39

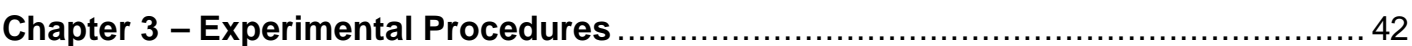

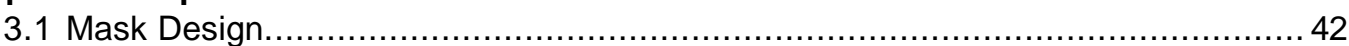

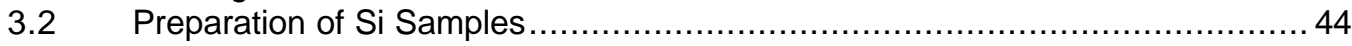

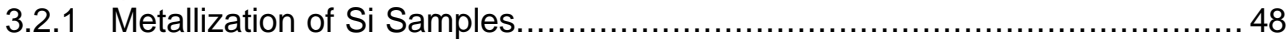

3.3 Preparation of GaN Samples ........................................................... 49

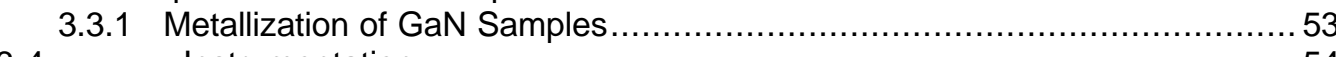

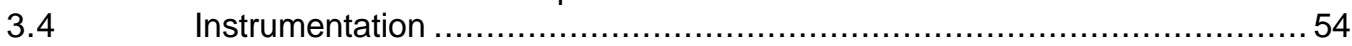

3.4.1 Instrumentation for C-V Measurements .......................................... 54

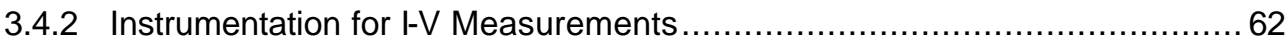

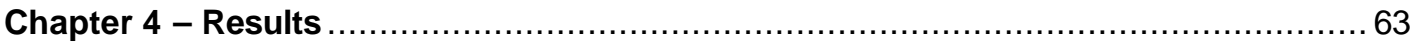

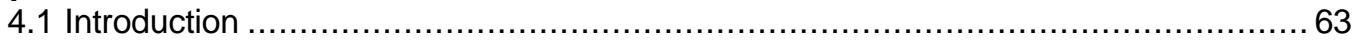

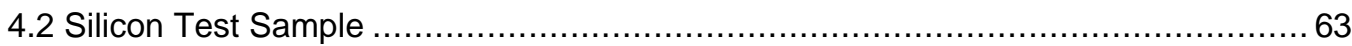

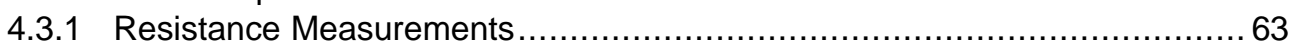

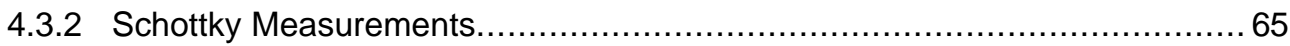

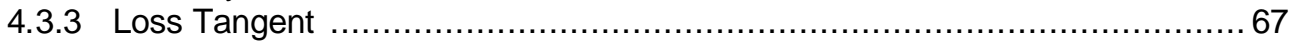

4.3.4 Depletion Capacitance at No Bias ..................................................... 68

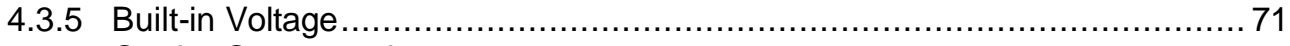

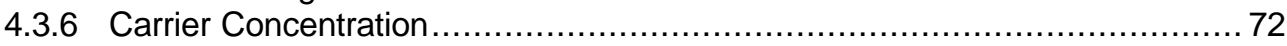

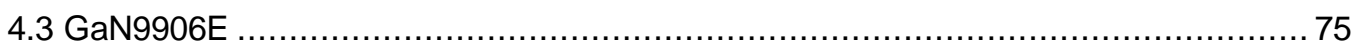

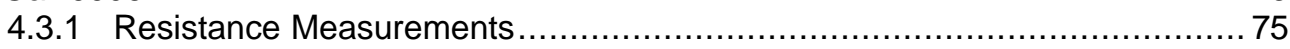

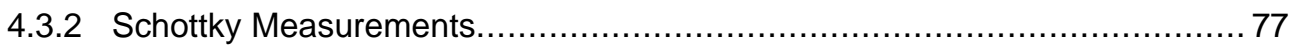

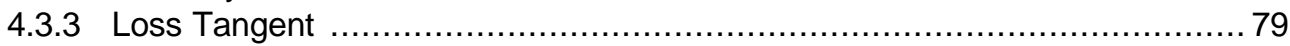

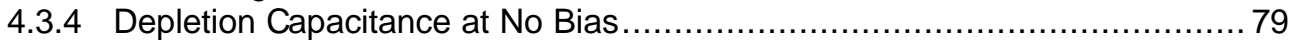

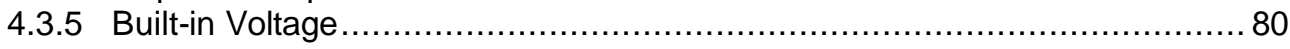

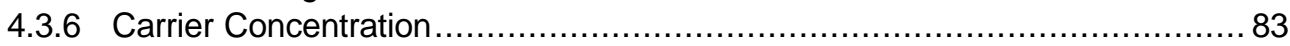




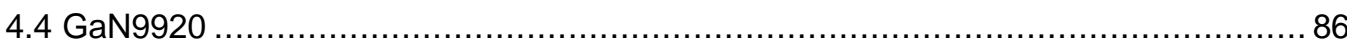

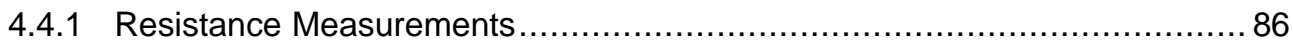

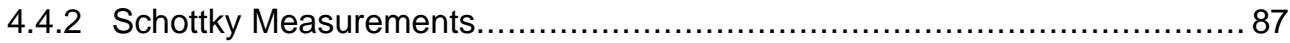

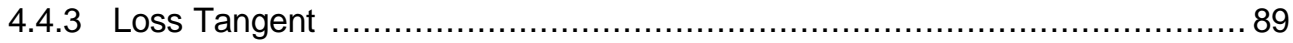

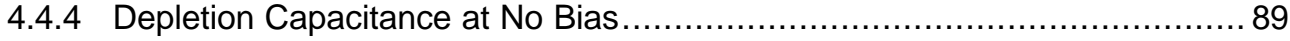

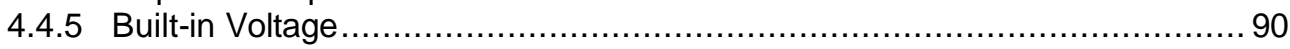

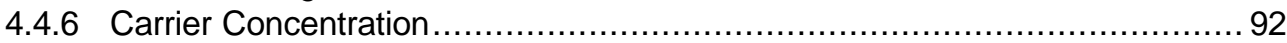

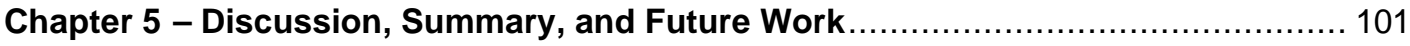

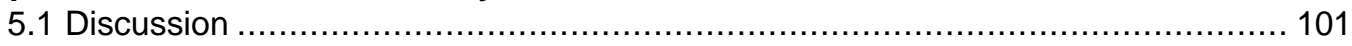

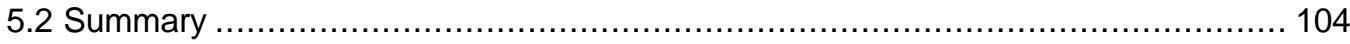

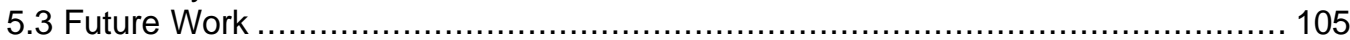

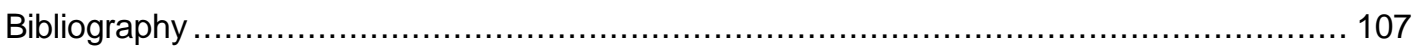

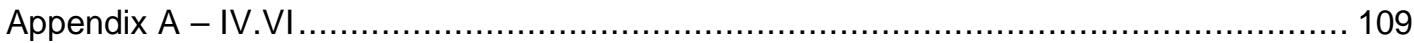

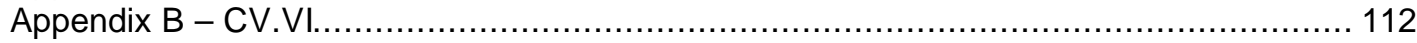

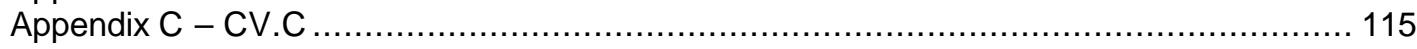

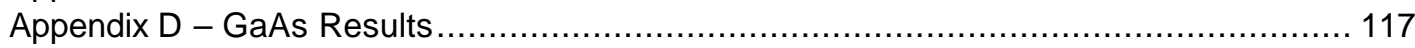

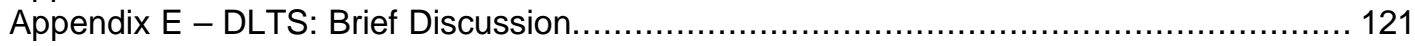

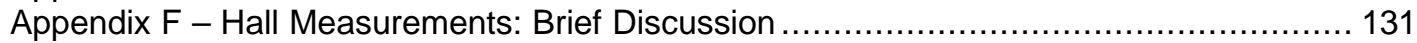


Figure 1.1: (a) Zincblende, and (b) Wurtzite Gallium Nitride structures ............................2

Figure 1.2: Typical Carrier Concentration Versus 1000 / Temperature for a silicon

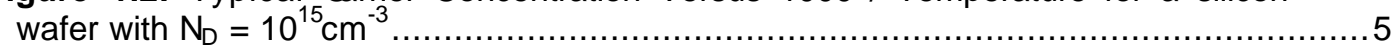

Figure 2.1: P-N junction and its charge density in the depletion region .............................. 6

Figure 2.2: (a) Depletion field, (b) Built-in potential, and (c) Resulting band diagram.............7

Figure 2.3: I-V characteristic of a pn junction......................................................... 9

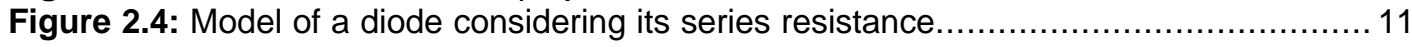

Figure 2.5: Work function of a metal................................................................. 15

Figure 2.6: Conduction, Valence, Fermi levels, and Electron affinity of a typical n-type

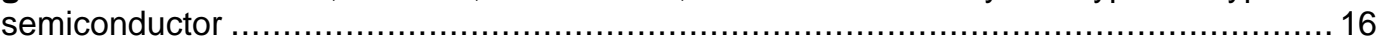

Figure 2.7: Band bending when a metal is placed in contact with a semiconductor for

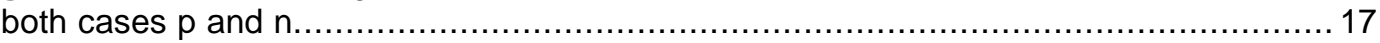

Figure 2.8: Parallel plate capacitor model of a pn junction........................................ 19

Figure 2.9: Depletion layer width and its ExK diagram at different polarizations ..................20

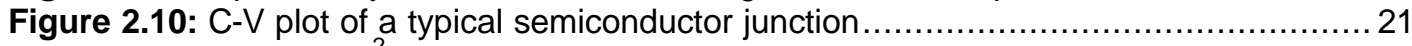

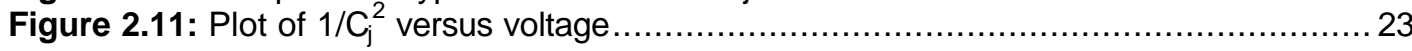

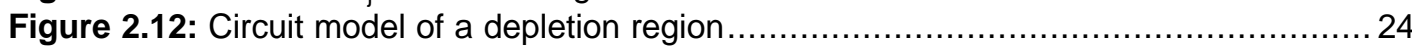

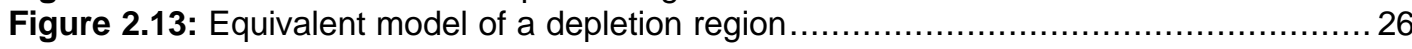

Figure 2.14: Typical plot of capacitance versus frequency of a junction..........................26

Figure 2.15: Typical plot of carrier concentration versus frequency of a junction................ 29

Figure 2.16: Circuit model of a depletion region including surface states, and neglecting the series resistance and the junction conductance.....................................2. 29

Figure 2.17: Stretch-out in the C-V curve due to interface-trapped charges ....................... 31

Figure 2.18: Phase diagram of an ideal depletion layer ............................................... 32

Figure 2.19: Typical plot of loss tangent versus frequency of a junction......................... 34

Figure 2.20: Electrical model of a depletion layer including a diffusion capacitance and

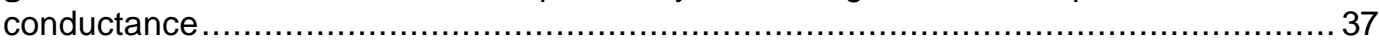

Figure 2.21: Diffusion capacitance and conductance as functions of frequency ................. 38

Figure 2.22: Total electrical circuit model of a depletion region ..................................... 38

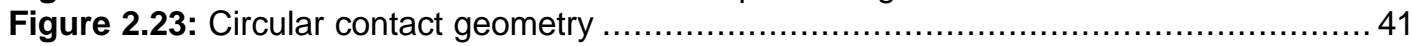

Figure 2.24: Voltage drop between contacts as a function of their separation ................... 41

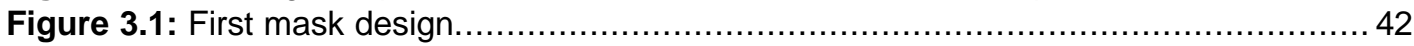

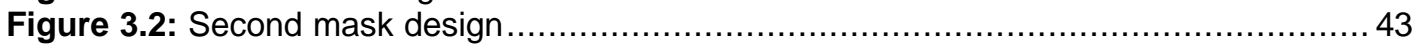

Figure 3.3: Flow diagram of preparation of silicon samples .................................... 44

Figure 3.4: Flow diagram of preparation of gallium nitride samples ............................ 50

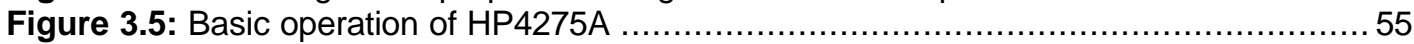

Figure 3.6: General Connection among the two HPs and the probing station for C-V

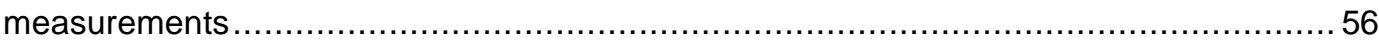

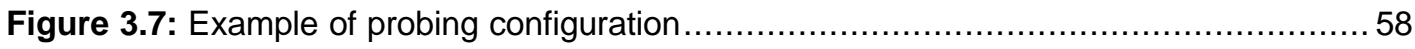

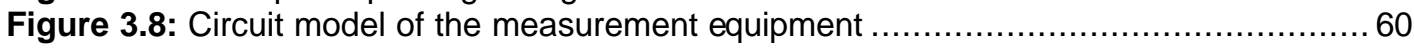

Figure 3.9: General connection between HP4140A and the probing station......................61

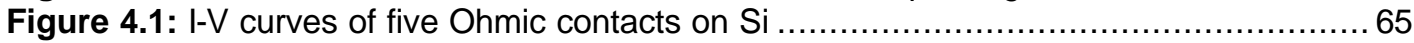

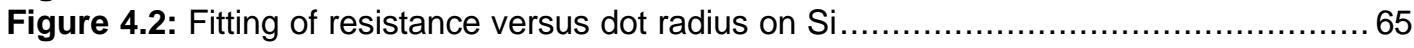

Figure 4.3: Log I-V plot of a $250 \mu \mathrm{m}$ dot with $20 \mu \mathrm{m}$ of separation on Si............................. 66

Figure 4.4: Saturation current of a $250 \mu \mathrm{m}$ dot with $20 \mu \mathrm{m}$ of separation on Si...................6 67

Figure 4.5: Loss tangent versus frequency of a $250 \mu \mathrm{m}$ dot with $20 \mu \mathrm{m}$ of separation on

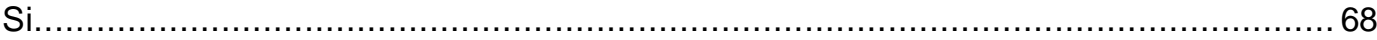

Figure 4.6: Junction capacitance measured on a $250 \mu \mathrm{m}$ dot with $20 \mu \mathrm{m}$ of separation

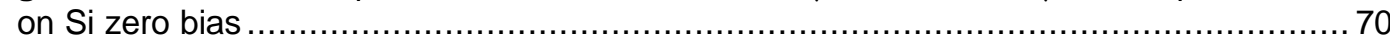


Figure 4.7: Junction conductance measured on a $250 \mu \mathrm{m}$ dot with $20 \mu \mathrm{m}$ of separation on $\mathrm{Si}$ at zero bias

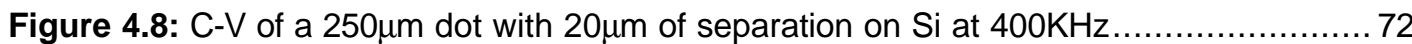

Figure 4.9: $\mathrm{C}^{-2}-\mathrm{V}$ of a $250 \mu \mathrm{m}$ dot with $20 \mu \mathrm{m}$ of separation on $\mathrm{Si}$ at $400 \mathrm{KHz} \ldots \ldots \ldots \ldots \ldots \ldots . . . \ldots \ldots$

Figure 4.10: $\mathrm{C}-\mathrm{V}$ curve of a contact on $\mathrm{Si}$ at different test frequencies........................... 74

Figure 4.11: Plot of carrier concentration of $\mathrm{Si}$ at different frequencies .......................... 75

Figure 4.12: I-V curves of four Ohmic contacts on GaN9906E ................................. 78

Figure 4.13: Fitting of resistance versus dot radius on GaN9906E ........................... 78

Figure 4.14: $\mathrm{Log} \mathrm{I} \mathrm{V}$ plot of a $250 \mu \mathrm{m}$ dot with $20 \mu \mathrm{m}$ of separation on GaN9906E .............. 79

Figure 4.15: Saturation current of a $250 \mu \mathrm{m}$ dot with $20 \mu \mathrm{m}$ of separation on GaN9906E...... 80

Figure 4.16: Loss tangent versus frequency of a $250 \mu \mathrm{m}$ dot with $20 \mu \mathrm{m}$ of separation

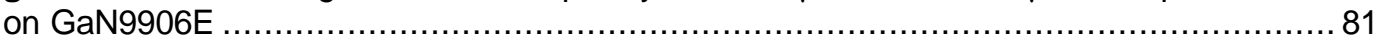

Figure 4.17: Junction capacitance of a $250 \mu \mathrm{m}$ dot with $20 \mu \mathrm{m}$ of separation at zero bias

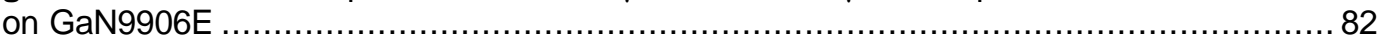

Figure 4.18: Junction conductance of a $250 \mu \mathrm{m}$ with $20 \mu \mathrm{m}$ of separation at zero bias on GaN9906E

Figure 4.19: $\mathrm{C}-\mathrm{V}$ plot of a $250 \mu \mathrm{m}$ dot with $20 \mu \mathrm{m}$ of separation at $20 \mathrm{KHz}$ on GaN9906E .....84

Figure 4.20: $\mathrm{C}^{-2}-\mathrm{V}$ of a $250 \mu \mathrm{m}$ dot with $20 \mu \mathrm{m}$ of separation at $20 \mathrm{KHz}$ on GaN9906E ...........8 84

Figure 4.21: C-V curve of a contact on GaN9906E at different test frequencies .................8 86

Figure 4.22: Plot of carrier concentration of GaN9906E at different frequencies ................ 87

Figure 4.23: I-V curves of five Ohmic contacts on GaN9920 .................................... 90

Figure 4.24: Fitting of resistance versus dot radius on GaN9920 ................................ 91

Figure 4.25: $\mathrm{Log} \mathrm{I} V \mathrm{~V}$ plot of a $250 \mu \mathrm{m}$ dot with $20 \mu \mathrm{m}$ of separation on GaN9920 ................ 92

Figure 4.26: Saturation current of a $250 \mu \mathrm{m}$ dot with $20 \mu \mathrm{m}$ of separation on GaN9920........ 92

Figure 4.27: Loss tangent versus frequency of a $250 \mu \mathrm{m}$ dot with $20 \mu \mathrm{m}$ of separation on GaN9920

Figure 4.28: Junction capacitance of a $250 \mu \mathrm{m}$ dot with $20 \mu \mathrm{m}$ of separation at zero bias on GaN9906E

Figure 4.29: Junction conductance of a $250 \mu \mathrm{m}$ dot with $20 \mu \mathrm{m}$ of separation at zero

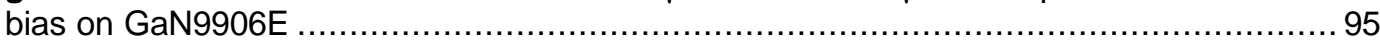

Figure 4.30: $\mathrm{C}-\mathrm{V}$ of a $250 \mu \mathrm{m}$ dot with $20 \mu \mathrm{m}$ of separation at $400 \mathrm{KHz}$ on GaN9920 ........... 97

Figure 4.31: $\mathrm{C}^{-2}-\mathrm{V}$ of a $250 \mu \mathrm{m}$ dot with $20 \mu \mathrm{m}$ of separation at $400 \mathrm{KHz}$ on GaN9920..........97

Figure 4.32: C-V curve of a contact on GaN9920 at different frequencies........................98

Figure 4.33: Plot of carrier concentration of GaN9920 at different frequencies .................. 99

Figure 5.1: Carrier concentration as a function of the dot radius ............................... 102

Figure 5.2: Variation of the depletion width with respect to the applied voltage as a

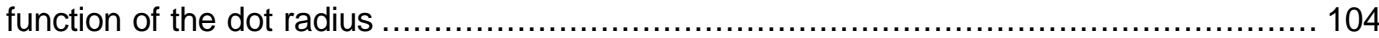

Figure 6.1: Sequence of occupation of majority-carrier traps .................................... 118

Figure 6.2: Capacitance transient of a junction due to an applied pulse........................ 119

Figure 6.3: DLTS Transients at different temperatures with a fixed time window .............. 120

Figure 6.4: Typical plot of the normalized DLTS signal versus temperature. .................... 121

Figure 6.5: Typical log plot of the thermal emission rate versus 1000/T. ..................... 122

Figure 6.6: General connection among instruments for DLTS measurements................. 122

Figure 6.7: Typical plot of standard deviation versus temperature for a DLTS

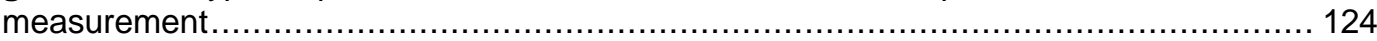

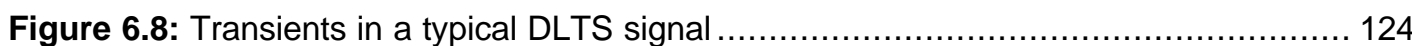

Figure 6.9: Typical relative capacitances versus temperature in a DLTS measurement .... 125

Figure 6.10: Typical sample with contacts on its corners, and connections diagram ......... 131 


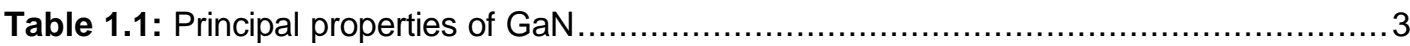

Table 2.1: Points to be observed during measurements related to a depletion region ..........39

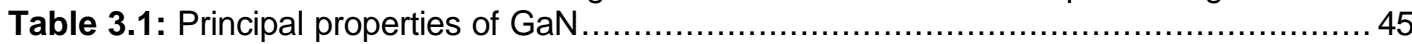

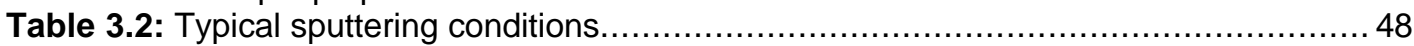

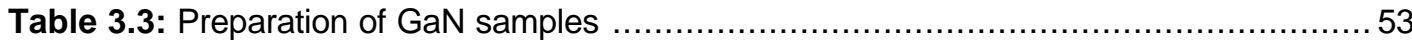

Table 4.1: Total resistance versus radius of five Ohmic contacts on silicon......................64

Table 4.2: Points of lowest losses for the measured contacts on Si............................... 70

Table 4.3: Slope and intercept of $\mathrm{C}^{-2}-\mathrm{V}$ curves of four contacts on Si........................... 71

Table 4.4: Calculated built-in voltages for the measured contacts on Si.......................... 72

Table 4.5: Carrier concentration of $\mathrm{Si}$ at different frequencies .................................... 75

Table 4.6: Total resistance versus radius of five Ohmic contacts on GaN9906E ................77

Table 4.7: Points of lowest losses for the measured contacts on GaN9906E ..................... 81

Table 4.8: Slope and intercept of $C^{-2}-V$ curves of four contacts on GaN9906E ................. 83

Table 4.9: Calculated built-in voltages for the measured contacts on GaN9906E ............... 83

Table 4.10: Corrected built-in voltages for contacts on GaN9906E ............................... 85

Table 4.11: Carrier concentration of GaN9906E at different frequencies............................ 87

Table 4.12: Adjusted values of the carrier concentration for GaN9906E ........................ 88

Table 4.13: Total resistance versus radius of five Ohmic contacts on GaN9920 ................ 89

Table 4.14: Points of lowest losses for the measured contacts on GaN9920 ..................... 94

Table 4.15: Slope and intercept of $C^{-2}-V$ curves of four contacts on GaN9920 .................. 95

Table 4.16: Calculated built-in voltages for the measured contacts on GaN9920 ................96

Table 4.17: Corrected built-in voltages for the measured contacts on GaN9920 ................ 96

Table 4.18: Carrier concentration of GaN9920 at different frequencies ............................. 98

Table 4.19: Adjusted values of the carrier concentration for GaN9920 .......................... 100

Table 5.1: Measured values of carrier concentration for different dot sizes....................... 101

Table 6.1: Measurement between a dot and a ring on GaAs .................................. 117

Table 6.2: Measurement between a dot and an Ohmic contact on backside of GaAs........ 117

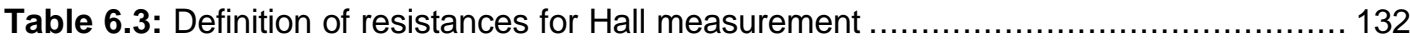

Table 6.4: Definition of characteristic resistances .................................................. 132

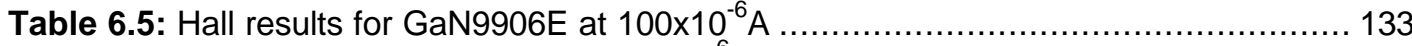

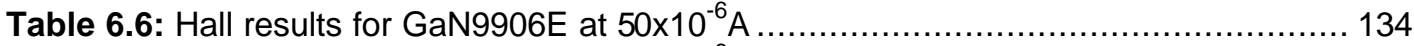

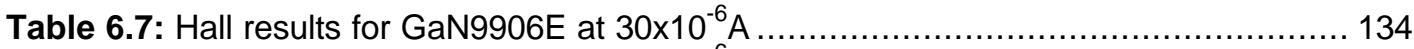

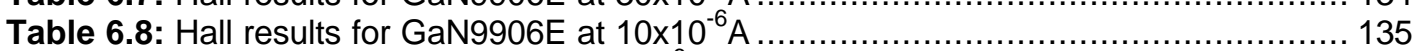

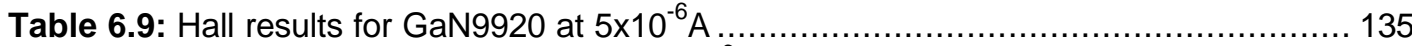

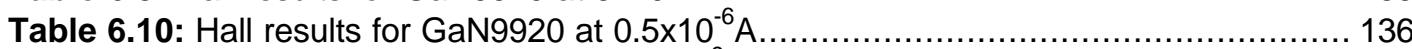

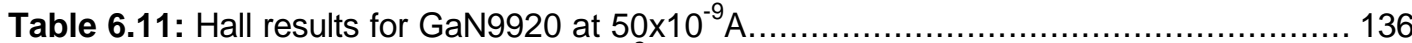

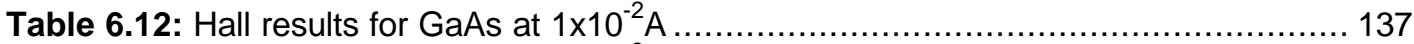

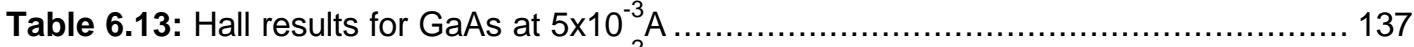

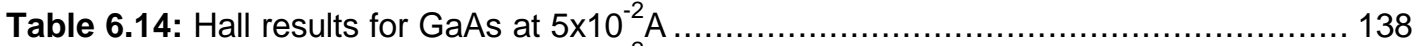

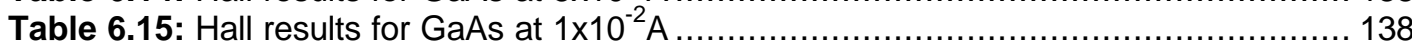

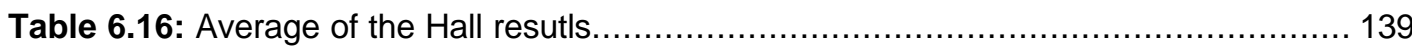




\section{Chapter 1-Introduction}

\subsection{HISTORICAL BACKGROUND}

For several years researchers have been trying to devise a material capable of emitting light in the blue region of the electromagnetic spectrum. The first researcher to successfully accomplish this task was J.Nakamura. Motivated by the idea of creating a high brightness light emitting diode (LED) that could emit blue light; this researcher started to investigate gallium nitride materials in 1983. Not much later, Nakamura devised the first gallium nitride based blue LED [1].

Due to its bandgap, GaN can also be used in space-based applications, since it has a high immunity to noise. Another typical application of wide bandgap materials is as power devices. Since the energy correspondent to the bandgap is usually bigger than $3 \mathrm{eV}$, these devices are a good solution for high operating current requirements.

Other than its electrical characteristics, $\mathrm{GaN}$ has also some other important features. Whereas typical III-V compounds are made of poisonous substances such as phosphate and arsenate (GaAs, InP), GaN is an entirely non-toxic semiconductor composed of safe elements that make the material environmental friendly.

\subsection{PHYSICAL PROPERTIES OF G aN}

Gallium nitride is composed of gallium, an element from the third column of the periodic table, and nitrogen, an element from the fifth column. This material is found in two crystal structures: zincblende and wurtzite [1].

This is particularly important for the growth of gallium nitride that is usually made on a sapphire substrate, which in turn has the same underlying hexagonal Bravais lattice of the wurtzite structure. Thus, this work will be restricted to the wurtzite grown gallium nitride. This 
is also due to the fact that all the samples used in this research were grown in this structure. Figure 1.1 shows the zincblende and the wurtzite gallium nitride structures.

In the case of the wurtzite gallium nitride, there are two lattice constants. Figure 1.1 shows the arrangement of the lattice constants. The constant 'c' indicates the height of the primitive cell, whereas the constant ' $\mathrm{a}$ ' indicates the separation among the atoms at the bottom plane of the primitive cell.

Typical theoretical values for 'a' and 'c' are $3.174 \AA$ and $5.169 \AA$ respectively [2], whereas typical experimental values are $3.189 \AA$ and $5.185 \AA$ [3]. For (1000) wurtzite gallium nitride grown on a (0001) sapphire substrate, a typical mismatch value is $16.1 \%$ with respect to the lattice constant ' $a$ ' [1].

In wurtzite gallium nitride, the bandgap at $0 \mathrm{~K}$ is approximately $3.5 \mathrm{eV}$ [3]. For the zincblende gallium nitride, the bandgap is usually a bit smaller. In either case, the bandgap is direct, as also found in other III-V semiconductors such as GaAs [1]. However, the bandgap of $\mathrm{GaN}$ is divided into three different energies de to splitting of degeneracy of its valence band [1]. These three transitions are usually labeled A, B, and C excitons. Table 1.1 shows the values of the main characteristics found in gallium nitride materials.

(a) Zincblende

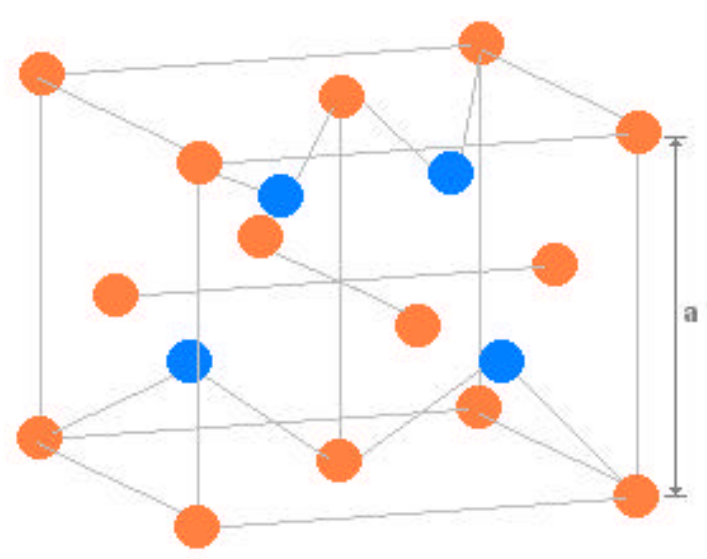

(b) Wurtzite

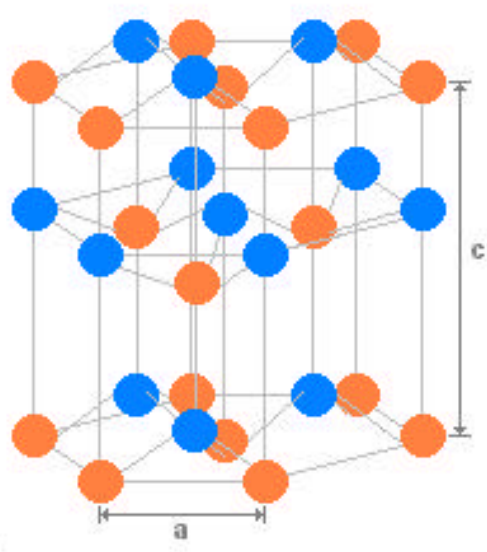

Ga or II

II or Ga

Figure 1.1: (a) Zincblende, and (b) Wurtzite Gallium Nitride structures. 
Table 1.1: Principal properties of GaN [1].

\begin{tabular}{|c|c|c|c|}
\hline $\mathrm{E}_{\mathrm{g}}(0 \mathrm{~K}),[\mathrm{eV}]$ & 3.5 & $\mathrm{~m}_{\mathrm{e}}^{*} / \mathrm{m}_{\mathrm{o}}$ & 0.2 \\
\hline $\mathrm{E}_{\mathrm{g}}(300 \mathrm{~K}),[\mathrm{eV}]$ & 3.4 & $m_{h} * / m_{0}$ & 0.8 \\
\hline$\Delta \mathrm{E}_{\mathrm{ab},}[\mathrm{eV}]$ & $6 \times 10^{-3}$ & Refractive index $n$ & 2.4 \\
\hline$\Delta \mathrm{E}_{\mathrm{bc},}[\mathrm{eV}]$ & $37 \times 10^{-3}$ & Static dielectric constant $\in$ & 12 \\
\hline Lowest cond.-band minimum & $0000-\operatorname{dir}$ & Lattice constant $\boldsymbol{a}, \boldsymbol{c}[\AA] \AA$ & $3.18,5.16$ \\
\hline$\left(\mathrm{dE}_{\mathrm{g}} / \mathrm{dT}\right) \times 10^{4} @ 300 \mathrm{~K},[\mathrm{eV} / \mathrm{K}]$ & -4.8 & $\mu_{\mathrm{e}}\left[\mathrm{cm}^{2} / \mathrm{V}_{\mathrm{xsec}}\right]$ & 300 \\
\hline$(\mathrm{dE} / \mathrm{dP}) \times 10^{6},[\mathrm{eV} / \mathrm{bar}]$ & 4.2 & $\mu_{\mathrm{h}}\left[\mathrm{cm}^{2} / \mathrm{V}_{\mathrm{xSec}}\right]$ & NA \\
\hline
\end{tabular}

\subsection{STATEMENT OF THE PRO BLEM}

Gallium nitride, as addressed before, is a very important material for the areas of optoelectronics and power-electronics due to its wide bandgap. However, the growth of this material needs to be better understood. The basic growth of $\mathrm{GaN}$ results in an unintentionally $\mathrm{n}$ type doping, usually in the order of $10^{16}-10^{17} \mathrm{~cm}^{-3}$. A better growth has to be devised so that purer materials can be achieved. Also, the growth of good quality ptype gallium nitride has to be capable to compensate the undesired donors, and inject more acceptors in order to achieve acceptable doping concentrations.

Molecular Beam Epitaxy (MBE) is a technique that provides precise control of the growth conditions, and is hence a good methodology to use to produce pure study samples. Metallorganic Chemical Vapor Deposition (MOCVD) is another growth technique used to grow gallium nitride. Its main advantage over MBE is its faster growth time. Even if MOCVD growth is preferred for commercial scale production, firstly the growth of $\mathrm{GaN}$ has to be well understood and MBE is employed to produce the study samples.

The problem presented in this thesis is the basic understanding and development of tools that can provide feedback to the growth of high-quality gallium nitride samples. When designing devices, one is usually interested in having the carrier concentration constant over a wide range of temperatures so that the resulting circuit has the same response despite the change in temperature. In general, solid state devices have three distinct regions in their carrier concentration $v s$. temperature curve. 
The best way of analyzing these three regions is by considering an extrinsic semiconductor, i.e. a material where the impurities contribute significantly to the total number of conduction carriers. In this case, the saturation range is the region of interest for device design, since the carrier concentration remains stable over a wide range of temperatures. The second region, at high temperatures, is the intrinsic range where the density of electrons is approximately the same as the density of holes, and both are much bigger than the density of impurities $\left(\mathrm{n} \approx \mathrm{p}>\mathrm{N}_{\mathrm{D}}\right.$ ). The third one, at very low temperatures, is the freeze-out range where most of impurities are frozen out. Figure 1.2 illustrates these three regions for the case of a typical silicon wafer.

When growing intrinsic gallium nitride, one is then interested in obtaining the smallest number possible of impurities on its bulk. On the other hand one is interested in controlling the doping when growing intentional n-type or p-type gallium nitride samples. This control is performed so that the resulting device has its saturation range at appropriate temperatures.

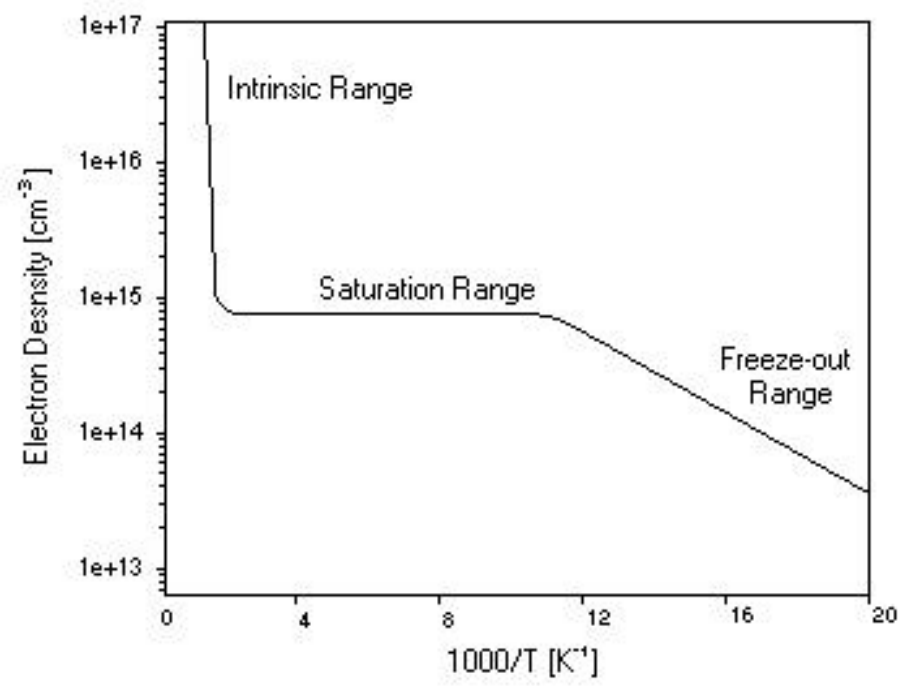

Figure 1.2: Typical Carrier Concentration versus 1000 / Temperature for a silicon wafer with $\mathrm{N}_{D}$ $=10^{15} \mathrm{~cm}^{-3}$. 
Measuring the carrier concentration is then of great importance in providing feedback for the growth of samples. Two main methods can be used to measure the carrier concentration. One method is Hall measurement (Appendix E), and the second method is by measuring the capacitance vs. voltage curve. This thesis focuses in the second method and will discuss it further in the following chapters.

Some problems arise when one tries to create and characterize a depletion region in highly resistive materials. The low doping and therefore the high resistivity of these materials include a set of nonidealities in the standard model of a depletion region. These nonidealities have to be properly modeled in order to perform an accurate measurement.

This thesis deals with the development of experimental and theoretical foundations for the measurement of capacitance, built-in voltage, and carrier concentration using capacitancevoltage measurements. The theoretical background necessary to understand the electrical circuits used to model the depletion region is presented in chapter 2. Chapter 3 deals with the experimental setup necessary to performed accurate measurements based on the theoretical background presented. Measurements performed on silicon to emulate the behavior of unintentionally doped n-type gallium nitride, and measurements performed in two samples of $\mathrm{GaN}$ are presented in chapter 4 . Concluding this thesis, chapter 5 contains final discussions, a summary of the work developed, and suggestions for future work. 


\section{Chapter 2-Theoretical Background}

\subsection{GENERAL SEMICONDUCTOR PHYSICS}

Schottky diodes can be understood as $\mathrm{p} n$ junctions in which one of the semiconductors is replaced by a metal. This metal in turn, behaves as a heavily doped material playing the role of one side of the junction. For example, in the case of a p-type wafer of silicon it is possible to create a $\mathrm{p} n$ junction by ion implanting an ntype region on it. A similar result can be achieved if instead of this implantation, a metal with appropriate work function is deposited onto the surface of this wafer.

To make the understanding of contact processing easy, the ideal $\mathrm{pn}$ junction is analyzed first. When both $\mathrm{p}$ and $\mathrm{n}$ materials are placed in contact, diffusion current is expected to appear in the junction due to the strong difference in charge concentrations. Therefore p-type carriers (holes) diffuse into the n-type side of junction and n-type carriers (electrons) migrate to the $\mathrm{p}$ type side of the junction creating a depletion region at the interface of the junction. Equilibrium is reached when an electric field is created by the accumulation of charges in the interface of the junction. This electric field generates a drift current in the opposite direction of the diffusion current in order to maintain the net current equal to zero in the junction. Figure 2.1 shows a condition when a p-type semiconductor is put in contact with an n-type semiconductor. In the graph, it is assumed that the number of acceptors $\left(\mathrm{N}_{\mathrm{A}}\right)$ in the material is much higher than the number of donors $\left(\mathrm{N}_{\mathrm{D}}\right)$, and therefore the depletion region is moved towards the $\mathrm{n}$-side of the junction.

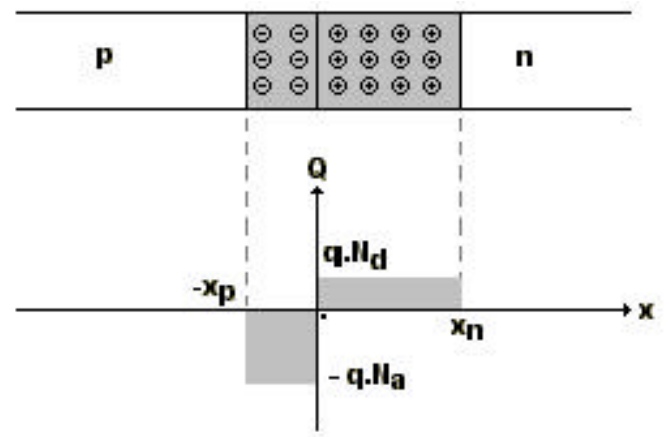

Figure 2.1: $\mathrm{P}-\mathrm{N}$ junction and its charge density in the depletion region. 
It is assumed that the charge density is $-\mathrm{qN}_{\mathrm{A}}$ in the negative side of the depletion region, $\mathrm{qN}_{\mathrm{D}}$ in the positive side, and it drops abruptly to zero outside the junction. The charge of an electron is denoted by $\mathrm{q}$ in the graph.

The electric field created in the depletion region (E) is directly proportional to the ntegral of the charge distribution along the x-axis. Considering the abrupt approximation, the electric field has a triangular shape. The electric potential, in turn, is the integral of the electric field along the $\mathrm{x}$-axis, as shown in equation 2.1.

$$
V=-\int \mathbf{E} \cdot d x
$$

Given any potential, the energy related to it is expressed by the potential divided by the electronic charge q. This is expressed by equation 2.2 .

$$
E=-\frac{V}{q}
$$

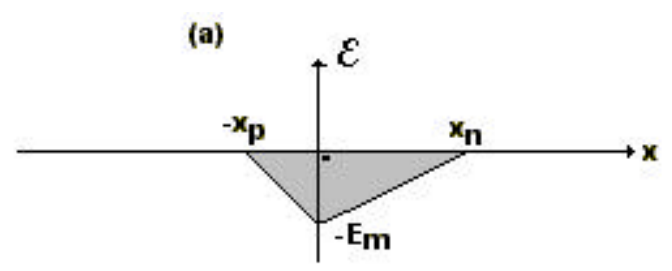

(b)

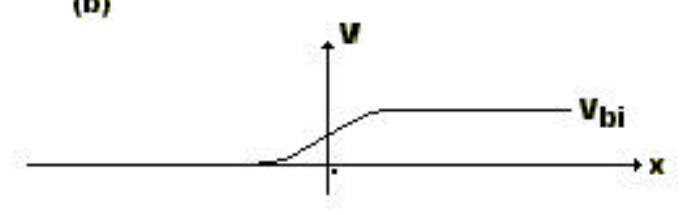

(c)

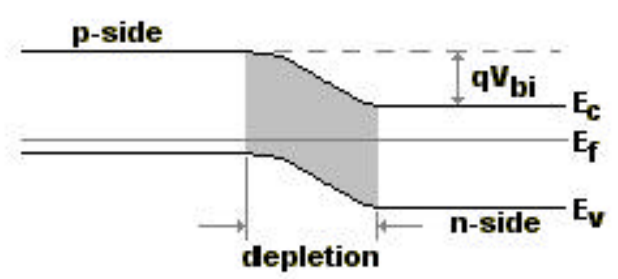

Figure 2.2: (a) Depletion field, (b) Built-in potential, and (c) Resulting band diagram. 
Hence, the band diagram resulting from a $\mathrm{p}$ material placed in contact with an $\mathrm{n}$ material has a smooth curve as shown in figure 2.2. In this figure, E is the energy at the bottom of the conduction band, $\mathrm{E}_{\mathrm{F}}$ is the energy at the top of the valence band, $\mathrm{F}_{\mathrm{f}}$ is the Fermi level, and $\mathrm{V}_{\mathrm{bi}}$ is the built-in voltage resulting from the distribution of bound charges in the junction. From this diagram it is easy to see how the carriers flow in the junction in a presence of an applied external field. When reverse biasing the junction, making its 1 side positive with respect to the $p$ side, the right side of the band is lowered and the potential that electrons have to overcome to flow to the $\mathrm{p}$-side of the junction is increased. Thus, the electric current, that has the opposite direction of the flow of electrons, is energetically unfavorable. On the other hand, if the pside of the junction is made positive in respect to the n-side, the junction is forward biased, the barrier potential is lowered, and flow of electrons from the p-side to the n-side is favored. If this applied potential happens to be near the barrier potential, the electrons in the n-type of the region have enough energy to flow towards the p-type. Correspondingly, an electric current exists from the ptype to the n-type side of the junction.

A good way to visualize the electronic behavior of a p-n junction is by taking a current vs. voltage (I-V) plot of the $\mathrm{pn}$ junction. In order to take his measurement, a voltage source is connected to the pside of the junction and the $n$ side is connected to ground. Varying the voltage from a negative value to a positive value one can visualize the influence of the junction potential. For negative voltages, the p-side has its energy level raised and the transport of electrons from the anode towards the cathode is ideally blocked. However, when the voltage is made positive and approaches the barrier potential, the energy level of the cathode is lowered to a level at which electrons can freely travel from the $\mathrm{n}$ to the $\mathrm{p}$ side of the junction and then current occurs. This behavior is illustrated in figure 2.3.

Two main points are shown in figure 2.3. The first is the threshold voltage $\left(\mathrm{V}_{\mathrm{th}}\right)$. The threshold voltage is the voltage at which significant conduction from the $\mathrm{p}$-side to the $\mathrm{n}$-side of the junction starts to occur. At any voltage above the threshold voltage, the ideal voltage drop across the diode is zero. In practice the diode behaves as a short circuit and the current that flows through it is limited, among other things, by its series resistance. 


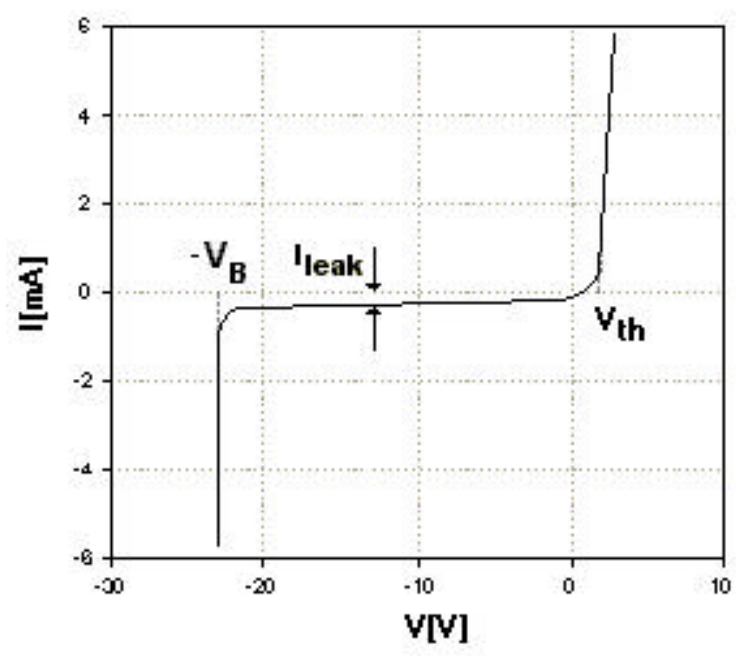

Figure 2.3: I-V characteristic of a p-n junction.

A typical value for the threshold voltage, in silicon for example, is $0.7 \mathrm{~V}$. Experimentally this value is given by analyzing the I-V curve. The threshold voltage is the point of the extrapolated forward part of the I-V curve that intersects the voltage axis. This intersection provides the threshold voltage. It is also important to note that the forward part of the curve has a slope. This is due to the fact that the real $\mathrm{pn}$ diode has a series resistance associated to it. This resistance is generally associated with the bulk resistance of the materials used.

Another important point indicated on figure 2.3 is the leakage current. An analysis of the leakage current can be carried out the following manner. A reverse biased junction still has a current flowing through it due to minority carriers. Although electrons in the n-side of the junction see a barrier when approaching the p-side, electrons located in the p-side see a huge drop of energy when moving to the rside of the junction. These electrons located in the $p$ side, are minority carriers, but nonetheless, contribute to a current from the n-side to the $\mathrm{p}$-side at a reverse applied bias in the junction. For example, when a negative voltage is applied to the pside, its energy is raised and this creates an energy barrier for electrons flowing from the $n$ towards the $\mathrm{p}$ region of the junction. However, the $\mathrm{p}$-side also has minority electrons that can energetically fall to the $\mathrm{n}$-side. This current is called leakage current and is found for negative values of the $\mathrm{I}-\mathrm{V}$ curve. 
The leakage current $\left(\mathrm{I}_{0}\right)$ and the forward currents are related by the Shockley equations [5]. Solving the Shockley equations for a diode one finds that the total net current found in the junction is given by equation 2.3 .

$$
I=I_{o} \cdot\left[\exp \left(\frac{q \cdot V_{a}}{k_{B} \cdot T}\right)-1\right]
$$

In equation $2.3, \mathrm{~b}$ is the leakage current, $\mathrm{q}$ is the electron charge $\left(1.6 \times 10^{-19} \mathrm{C}\right), \mathrm{V}_{\mathrm{a}}$ is the applied voltage, $\mathrm{k}_{\mathrm{B}}$ is the Boltzmann constant, and $\mathrm{T}$ is the temperature of the junction. This equation assumes an ideal $\mathrm{p}-\mathrm{n}$ junction and says that the transition from the reverse to the forward condition occurs in an exponential smooth manner. Assuming now a junction with a series resistance, as shown in figure 2.4 , one finds that the total resistance found in this diode is given by equation 2.4 .

$$
R_{t}=\frac{V a}{I}=\frac{V a}{I o \cdot\left[\exp \left(\frac{q \cdot V a}{k_{B} \cdot T}\right)-1\right]}+R_{S}
$$

Hence, the forward region of the I-V curve is divided into two parts. The first part is dominated by the exponential term of equation 2.4 , whereas the second part is dominated by the series resistance term. Therefore, for large values of the applied voltage, the dominant term in equation 2.4 is the series resistance.

If the junction is reverse biased and the applied voltage is sufficiently high, the minority carriers that participate in the leakage current may gain so much energy that they scatter atoms of the material and create new hole-electron pairs. This leads to a very large and multiplicative current. Due to this multiplicative behavior, the current after the breakdown voltage is called avalanche current. 


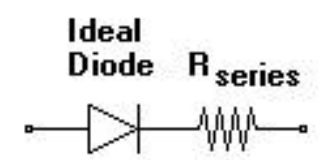

Figure 2.4: Model of a diode considering its series resistance.

The calculation of the breakdown voltage involves a quantum mechanical treatment that goes beyond the scope of this work. Nonetheless, an approximation is sometimes given by equation 2.5 [6], where $\mathrm{E}_{\mathrm{g}}$ is the bandgap of the material, and $\mathrm{N}$ is its carrier concentration.

$$
V_{B} \approx 60 \cdot\left(\frac{E_{g}}{1.1}\right)^{\frac{3}{2}}\left(\frac{N}{10^{16}}\right)^{-\frac{3}{4}}
$$

Another important characteristic of $\mathrm{p}-\mathrm{n}$ junctions is their depletion layer width. In order to find a mathematical expression for it, first the band diagram of the junction has to be analyzed further. Recalling figure 2.1, the Poisson equation has to be solved for that model with the purpose of finding the boundaries of the depletion charges region. Assuming that the charges are confined to the region indicated in figure 2.1, the Poisson equation leads to equation 2.6. In this equation $\mathrm{V}$ is the electric potential.

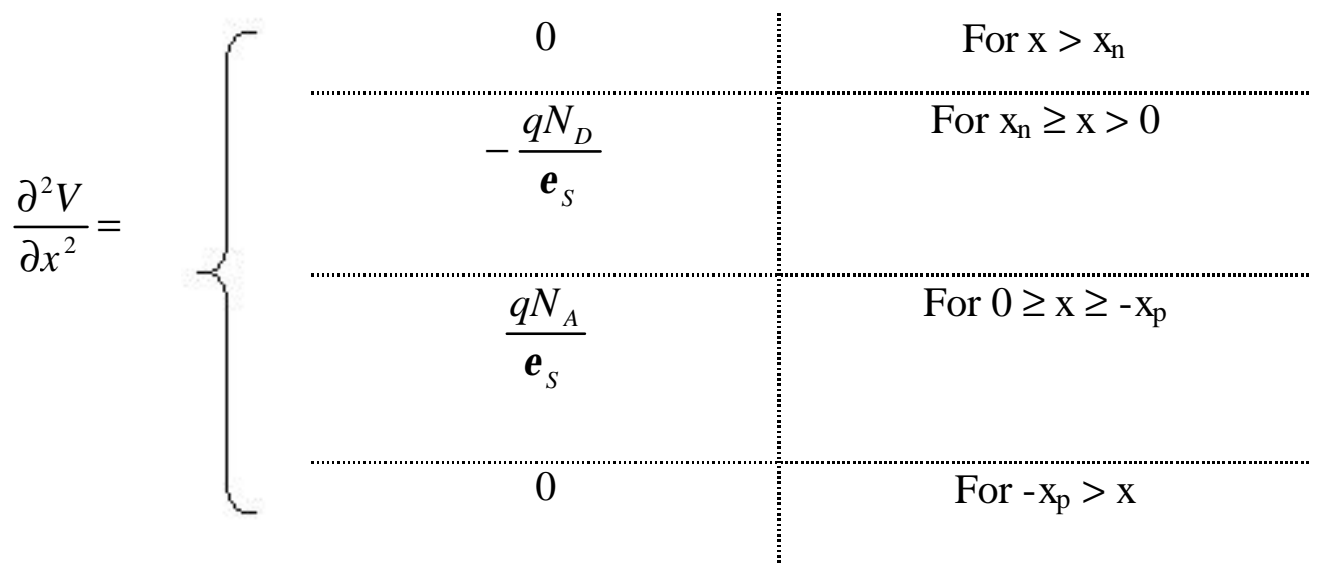

Equation 2.7 is then found by integrating equation 2.6 in respect to $\mathrm{x}$. In equation 2.7 the electric field is denoted by $\mathbf{E}$. 


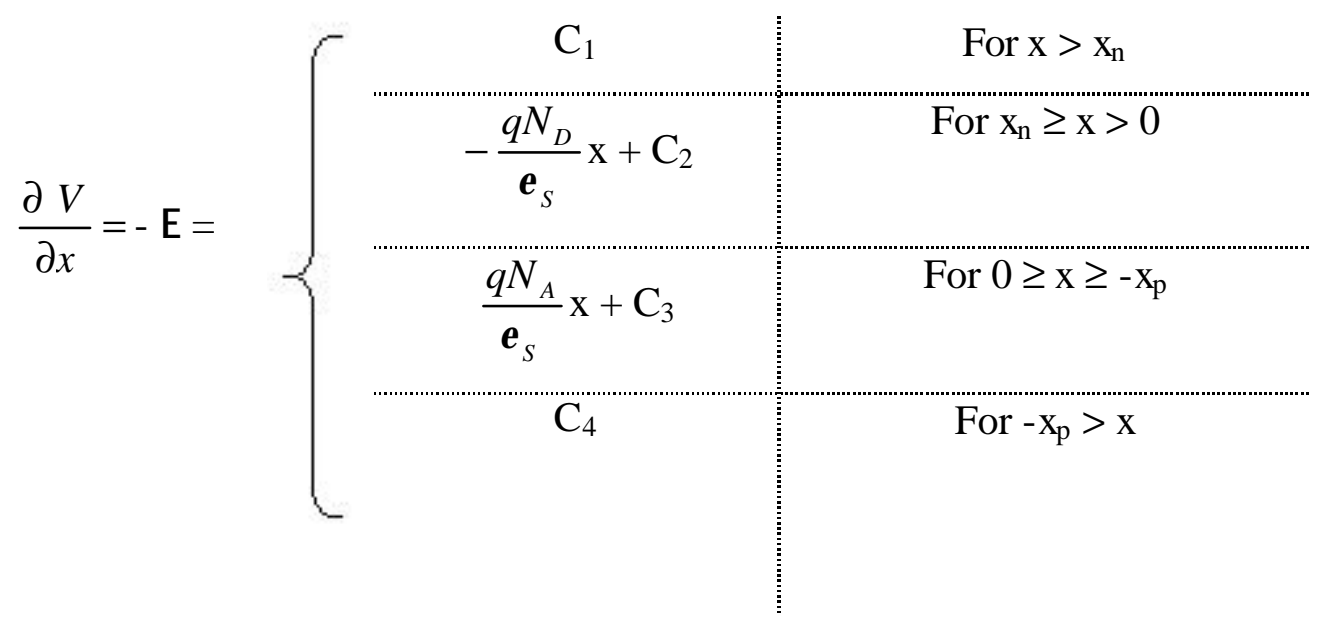

Remembering the model shown in figure 2.1, the electric field outside the depletion region has to be zero. Otherwise, there would be free carriers outside this region that would contribute to the existence of a net current. Since the junction has to be in equilibrium this current cannot exist and therefore the electric field outside the depletion region has to be zero. Thus, the constants $\mathrm{C}_{1}$ and $\mathrm{C}_{4}$ have to be zero. Also the electric field that exists inside the depletion region has to reach zero at the boundaries of the region. This condition leads to equations $2.8 \mathrm{a}$, and $2.8 \mathrm{~b}$.

$$
\begin{array}{lll}
\frac{q N_{D}}{\varepsilon_{S}} x_{n}+C_{2}=0 & \therefore & C_{2}=-\frac{q N_{D}}{\varepsilon_{S}} x_{n} \\
\frac{q N_{A}}{\varepsilon_{S}} x_{p}+C_{3}=0 & \therefore & C 3=-\frac{q N_{A}}{\varepsilon_{S}} x_{p}
\end{array}
$$

Including constants $\mathrm{C}_{1}, \mathrm{C}_{2}, \mathrm{C}_{3}$, and $\mathrm{C}_{4}$ into equation 2.7 , the electric field is then given by equation 2.9 . 


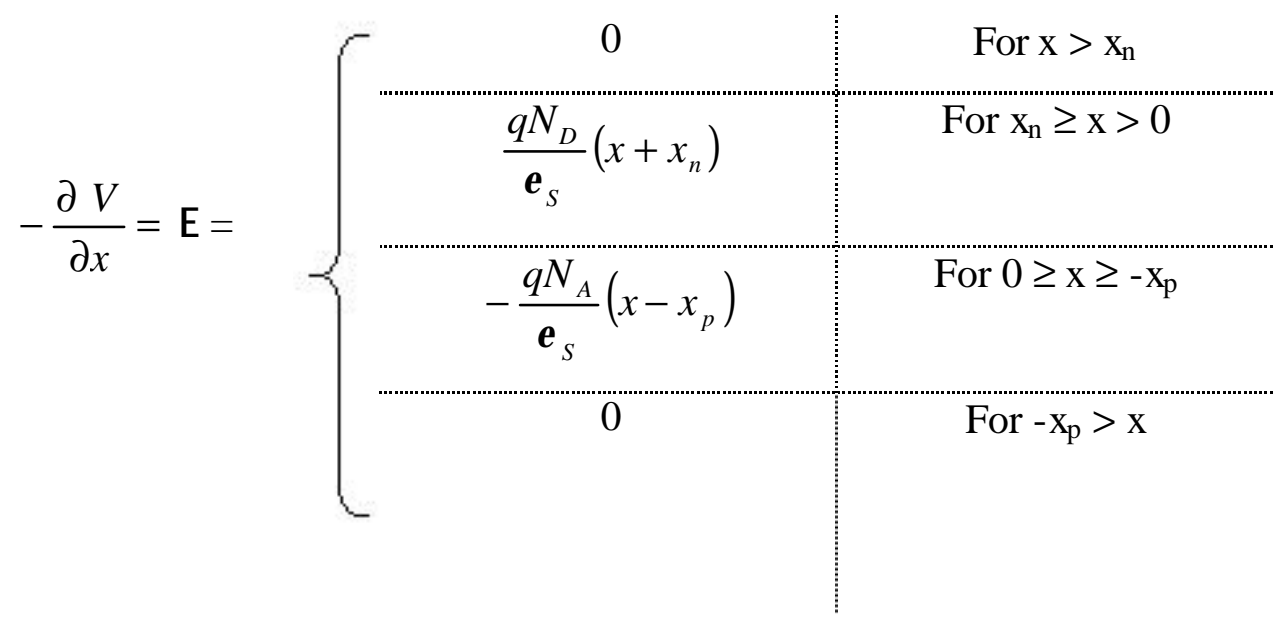

One important relation that can be extracted from equation 2.9 is the following. If one computes the electric field at the interface of the junction $(x=0)$, then equation $2.9 \mathrm{~b}$ relates the total charge per unit area in each side of the junction.

$$
N_{A} \cdot x_{p}=N_{D} \cdot x_{n}
$$

The next step towards finding an expression for the depletion region width is finding the potential distribution across the junction. Integrating equation 2.9 in respect to $\mathrm{x}$, one finds the potential profile given by equation 2.10 .

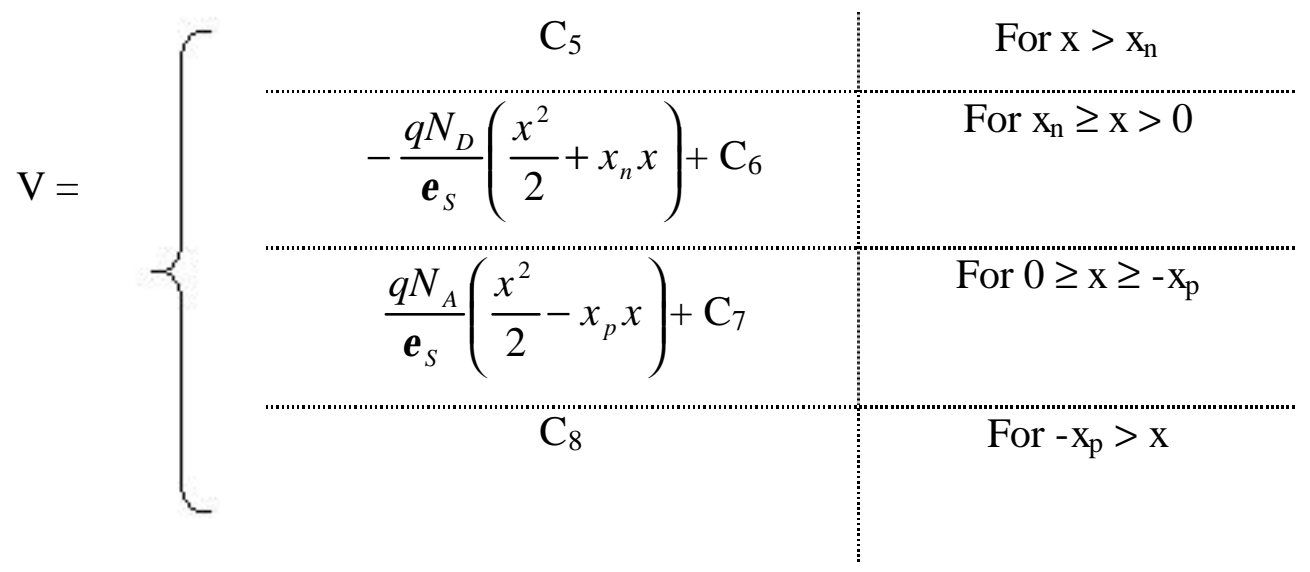

Recalling figure 2.1 and assuming that the p-side of the junction is the ground reference, this implies that $\mathrm{C}_{8}$ has to be zero. Also a built-in voltage across the junction has to appear because of the distribution of the electric field along its $\mathrm{x}$-axis. Therefore, $\mathrm{C}_{5}$ has to be the 
resulting built-in voltage $\mathrm{V}_{\mathrm{bi}}$ across the junction. Constants $\mathrm{C}_{6}$ and $\mathrm{C}_{7}$ can be easily found by applying the boundary conditions to potential equation. These conditions can be visualized by equations 2.11a, and 2.11b.

$$
\begin{array}{llc}
\frac{q N_{D}}{\varepsilon_{S}} \frac{x_{n}{ }^{2}}{2}+C_{6}=V_{b i} & \therefore & C_{6}=V_{b i}-\frac{q N_{D}}{\varepsilon_{S}} \frac{x_{n}{ }^{2}}{2} \\
-\frac{q N_{A}}{\varepsilon_{S}} \frac{x_{p}{ }^{2}}{2}+C_{7}=0 & \therefore & C 7=\frac{q N_{A}}{\varepsilon_{S}} \frac{x_{p}{ }^{2}}{2}
\end{array}
$$

Applying equations 2.11a, and 2.11b into equation 2.10, one finds the final expression for the electric potential across the junction. This expression is given by equation 2.12 .

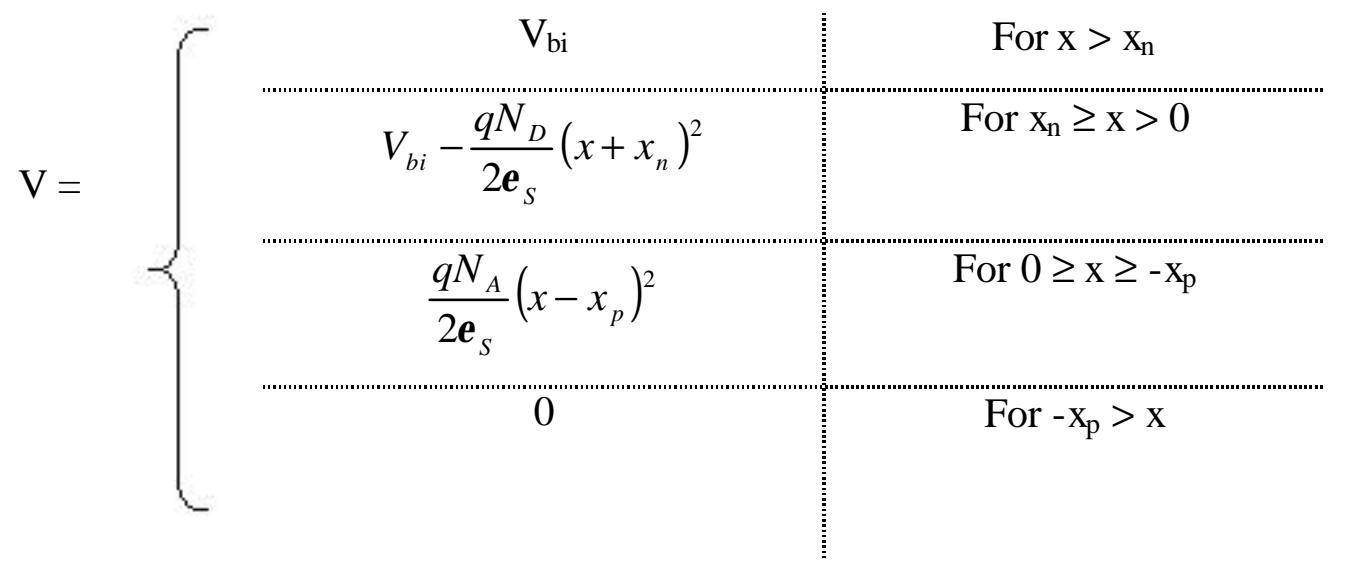

The depletion region width can then be found in the following way. The electric potential at the interface of the junction is given by equation 2.13 .

$$
V(0)=V_{b i}-\frac{q N_{D}}{2 \varepsilon_{S}} x_{n}{ }^{2}=\frac{q N_{A}}{2 \varepsilon_{S}} x_{p}{ }^{2}
$$

Analyzing equations 2.13 and $2.9 \mathrm{~b}$, and recalling figure 2.1 , it is easy to understand that if $\mathrm{N}_{\mathrm{A}}$ is much larger than $\mathrm{N}_{\mathrm{D}}$, the penetration of the depletion region into the $\mathrm{n}$-side of the junction is much larger than the penetration of the depletion region into the $\mathrm{p}$-side of the junction. In this situation, equation 2.13 can be rewritten as equation $2.14 \mathrm{a}$. 


$$
V_{b i}-\frac{q N_{D}}{2 \varepsilon_{S}} x_{n}^{2}=0
$$

Solving this last equation for the depletion width (W), one finds its expression that is given by equation $2.14 \mathrm{~b}$.

$$
W=x_{n}=\sqrt{\frac{2 \varepsilon_{S} \cdot V_{b i}}{q N_{D}}}
$$

\section{$2.2 \quad$ SCHOTTKY CONTACTS}

Another situation that may occur is when a metal replaces one side of a p-n junction. Depending on the carrier concentration of the semiconductor, work function of the metal, and other parameters, it is possible to have a situation where the metal plays the role of an extremely highly doped side of the junction.

The work function of a metal is defined as the energy necessary to remove an electron from the surface of this metal to free space. This energy level also specifies the upper-most energy state that is occupied by an electron in the metal. This definition can be visualized in figure 2.5 .

In the semiconductor side, for an ntype case, the conduction band is a region of energy where electrons have the possibility of occupation. The valence band is the next band below the conduction band where all states are already occupied by electrons. The Fermi level is defined as the energy of the topmost occupied level by an electron in the ground state of a semiconductor. The electron affinity of the semiconductor is defined as the energy difference between the topmost occupied state in the conduction band to the vacuum level. These definitions can be visualized in figure 2.6. 


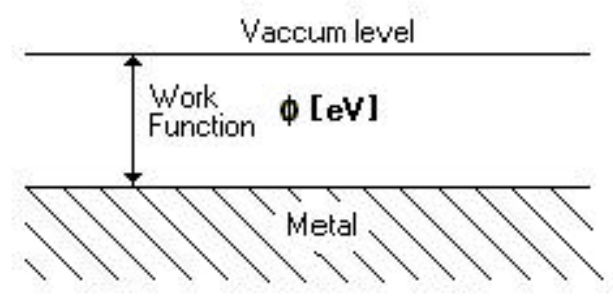

Figure 2.5: Work function of a metal.

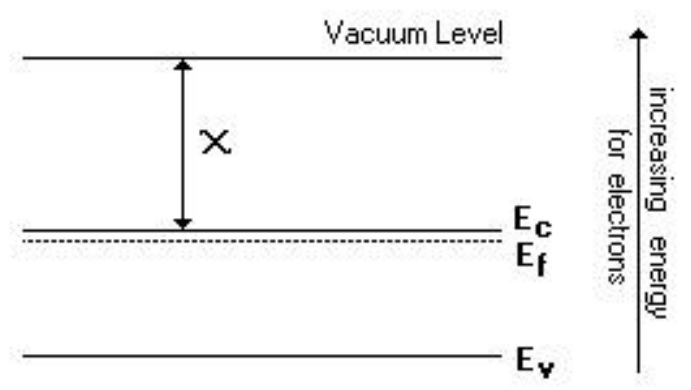

Figure 2.6: Conduction $\left(\mathrm{E}_{\mathrm{C}}\right)$, Valence $\left(\mathrm{E}_{\mathrm{V}}\right)$, Fermi $\left(\mathrm{E}_{\mathrm{F}}\right)$ levels, and Electron affinity $(\chi)$ of a typical n-semiconductor.

When the metal is put into contact with the semiconductor, some electrons of the semiconductor jump to the metal to restore energy equilibrium and therefore the Fermi level moves down from the conduction band in the semiconductor. This situation is known as band bending and is illustrated in figure 2.7. The conduction and valence bands maintain their values at the interface of the two materials. Consequently the barrier height created from the metal to the semiconductor has to be the difference between the work function of the metal and the electron affinity of the semiconductor. A similar analysis can be carried out for p-type semiconductors to find that the barrier height is given by the barrier height of the n-type case subtracted from the band gap of the semiconductor. The barrier heights of n-type and p-type semiconductors in contact with a metal are given by equations $2.15 \mathrm{a}$, and $2.15 \mathrm{~b}$.

$$
\begin{gathered}
\phi_{\text {bn }}=\phi-\chi \\
\phi_{\text {bp }}=\mathrm{E}_{\mathrm{g}}-(\phi-\chi)=\mathrm{E}_{\mathrm{g}}-\phi_{\mathrm{bn}}
\end{gathered}
$$




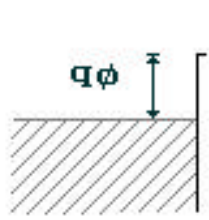

Hetal

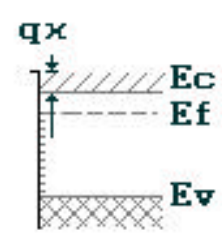

Semiconductor
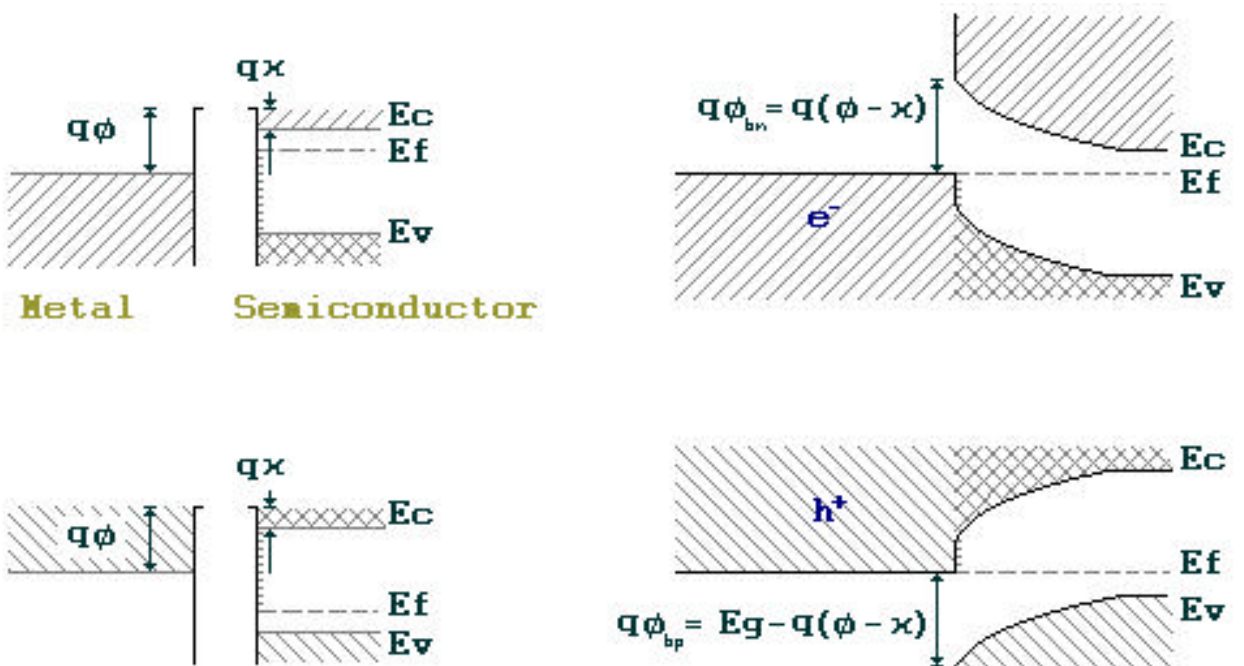

Hetal Semiconductor
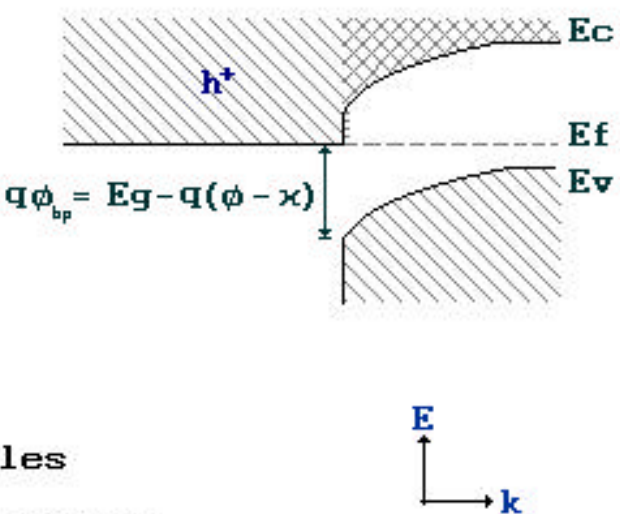

Available states for holes

Available states for electrons

80 Unavailable states

Figure 2.7: Band bending when a metal is placed in contact with a semiconductor for both cases $\mathrm{p}$ and $\mathrm{n}$.

The FV characteristic of a Schottky contact should be the same as of a $\mathrm{p}-\mathrm{n}$ junction, since there is a barrier potential from one to the other side of the junction. The only difference is that, in the case of Schottky contacts, the bands of the semiconductor are bent and the transition from one side to the other of the junction is more abrupt than in the case of $p-n$ junctions.

It is also important to note that there will always be a separation $(\delta)$ between the metal contact and the semiconductor bulk. In this case, for an n-type semiconductor bulk, equation $2.15 \mathrm{a}$ has to be replaced by equation $2.16 \mathrm{a}$.

$$
\begin{aligned}
& \phi_{B n}=c_{1} \cdot W+c_{2} \\
& c_{1}=\frac{\varepsilon_{0}}{\varepsilon_{0}+q^{2} \delta D_{S}}
\end{aligned}
$$




$$
c_{2}=\left(1-c_{1}\right) \cdot\left(\frac{E_{g}}{q}-\phi_{0}\right)
$$

In this last set of equations $E_{g}$ is the bandgap of the semiconductor, $D_{3}$ is the density of acceptor surface states, and $\phi_{0}$ is the energy level at the surface. The most valuable information provided by equation 2.16 is that in order to have the barrier height close to the ideal case (Eq. 2.15a) the separation between the metal and the surface of the semiconductor has to be small.

\subsection{CAPACITANCE VS. VOLTAGE ANALYSIS (C-V)}

As stated before, in a $\mathrm{pn}$ junction, due to the redistribution of charges, there is a region where charges are depleted. Considering a simple reverse biased $p-n$ junction, i.e. applying a positive voltage to its $\mathrm{n}$ side with respect to its $\mathrm{p}$-side, there will be a region close to the interface where electrons and holes are removed, leaving immobile ions in the junction. These ionized atoms carry a significant space charge so that there is a resulting high electric field at the interface of the junction. This region is called the depletion region due to its physical behavior. Since there is a redistribution of charges in this region and a voltage-dependent charge associated with the depletion region, there is also a capacitance associated with this junction.

A model that describes the simplest form of a depletion region of a junction is given by figure 2.8. This model is similar and has the same geometry of a parallel plate capacitor, where each side of the junction behaves as a plate of the capacitor. Applying the Gauss equation to this model one finds a dependence of the total charge (Q) in respect to the electric field (E) given by equation $2.17 \mathrm{a}$.

$$
\oint D \cdot d A=Q \quad \therefore \quad E=\frac{Q}{\varepsilon_{S} A}
$$

Then calculating the line integral for the resulting electric field, one finds the total voltage across the junction due to the space charge. This voltage is expressed in equation $2.17 \mathrm{~b}$. 


$$
\int^{W} E \cdot d l=V \quad \therefore \quad V=\frac{Q W}{\varepsilon_{S} A}
$$

Solving equation $2.17 \mathrm{~b}$ for the total charge $\mathrm{Q}$ and taking its derivative in respect to the total voltage $\mathrm{V}$, one finds an expression for the junction capacitance at zero bias. Combining this last expression with equation $2.14 \mathrm{~b}$ one finds the final expression for junction capacitance at zero bias. This result is given by equation $2.17 \mathrm{c}$.

$$
C_{j_{0}}=\frac{\partial Q}{\partial V}=\frac{\varepsilon_{S} A}{W}=A \sqrt{\frac{q \varepsilon_{S} N}{2 V_{b i}}}
$$

This derivation assumes that the edges of the depletion region are sharply defined as shown in figure 2.1, and that the voltage varies linearly across the junction. A more realistic view of the charge density assumes a smoother transition between the depletion region and the bulk of the materials. The error due to the difference between these two cases is given by two times the thermal voltage $\left(\mathrm{k}_{\mathrm{B}} \mathrm{T} / \mathrm{q}\right)[4]$.

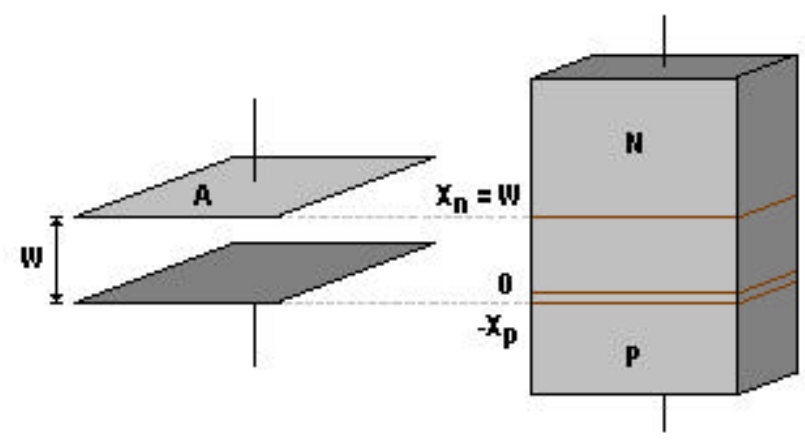

Figure 2.8: Parallel plate capacitor model of a p-n junction. 
Two cases shall be analyzed. The first case is when the junction is subjected to a forward bias, and the second case is when the junction is subjected to a reverse bias. In the first case the $\mathrm{p}$-side of the junction is polarized positively in respect to the r side. As the absolute value of the voltage starts to go up, holes migrate from the p-side of the junction to the depletion region. In the same way electrons migrate from the n-side of the junction to the depletion region. As a consequence of this injection, ions in the depletion region are neutralized and its width is decreased. Since capacitance is inversely proportional to the width of the depletion layer, as shown in equation $2.17 \mathrm{c}$, the capacitance of the junction is increased.

In the second case, the $\mathrm{n}$ side of the junction is made positive with respect to the $\mathrm{p}$-side. As the absolute value of the voltage starts to go up, holes are extracted from the depletion region and migrate to the p-region of the junction. In the same way electrons are extracted from the depletion region towards the n-region of the junction. Therefore, more atoms are ionized in the depletion region and its width is increased. Again, since capacitance is inversely proportional to the thickness, the capacitance of the junction is decreased. This situation where the width of the depletion region varies with the applied voltage is visualized in figure 2.9 with a metal playing the role of a heavily doped p-type semiconductor. 

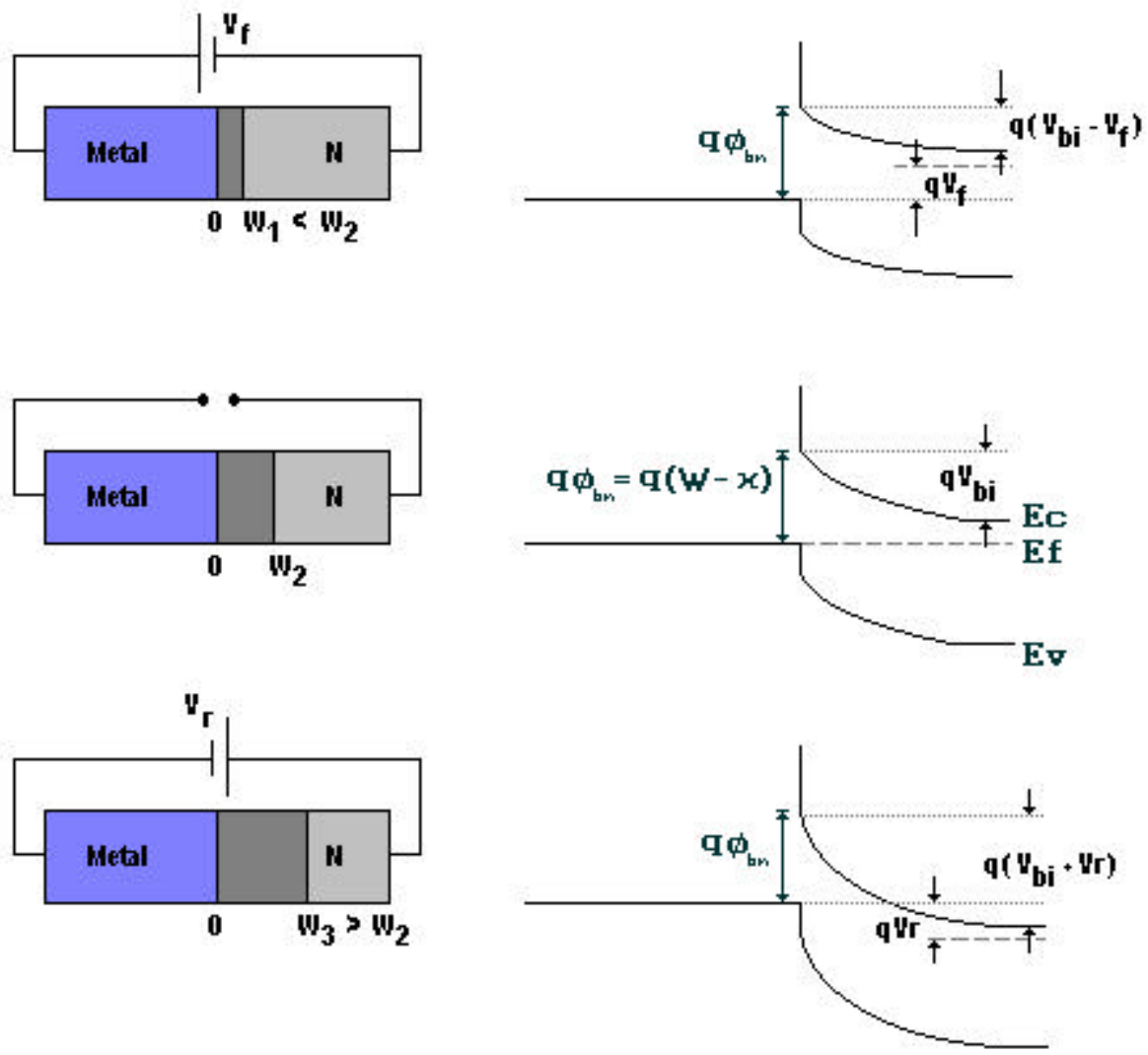

Figure 2.9: Depletion layer width and its ExK diagram at different polarizations.

Considering the graphical analysis found in figure 2.9 and the additional term brought from the error in the charge distribution, the expression for the junction capacitance at an applied voltage is given by equation $2.18 \mathrm{a}$. Combining this last equation with equation $2.17 \mathrm{c}$, the junction capacitance can also be expressed by equation $2.18 \mathrm{~b}$.

$$
\begin{gathered}
C_{j}=A \sqrt{\frac{q \varepsilon_{S} N}{2\left(V_{b i}-V-\frac{k_{B} T}{q}\right)}} \\
C_{j}=C_{j_{0}} \cdot \sqrt{\frac{1}{1-\frac{\left(V+\frac{k_{B} T}{q}\right)}{V_{b i}}}}
\end{gathered}
$$


A plot of equation $2.18 \mathrm{~b}$ is given by figure 2.10. It is observed in the figure that the capacitance tends to infinity for values of the voltage close to the built-in voltage. However, when approaches this limit, a high forward bias current flows through the junction, and the parallel plates capacitor model of the depletion is no longer valid. In figure 2.10 the dotted line shows the ideal behavior of equation $2.18 \mathrm{~b}$, whereas the solid line shows a more realistic behavior found in typical $\mathrm{C}-\mathrm{V}$ measurements. Acceptable values of capacitance are found for values of voltage up to half of the built-in voltage [7].

A valuable piece of information that can be extracted from $\mathrm{C}-\mathrm{V}$ curves is the carrier concentration of the analyzed material. It has been stated earlier in this work that when a heavily doped material is put into contact with a low-doped material, the carrier concentration $(\mathrm{N})$ found in previous derivations is that of the low-doped material. This is exactly the case when a metal is put into contact with a semiconductor, as discussed previously in session 2.2. From equation 2.18a it can be seen that the carrier concentration $(\mathrm{N})$ of the semiconductor can be easily found by some mathematical manipulations. First, a plot of $1 / C_{j}^{2}$ has to be taken. The region to be analyzed is the part of the curve situated between the breakdown voltage and half of the threshold voltage, where the small-signal model for the depletion region is valid. This plot is shown in figure 2.11 .

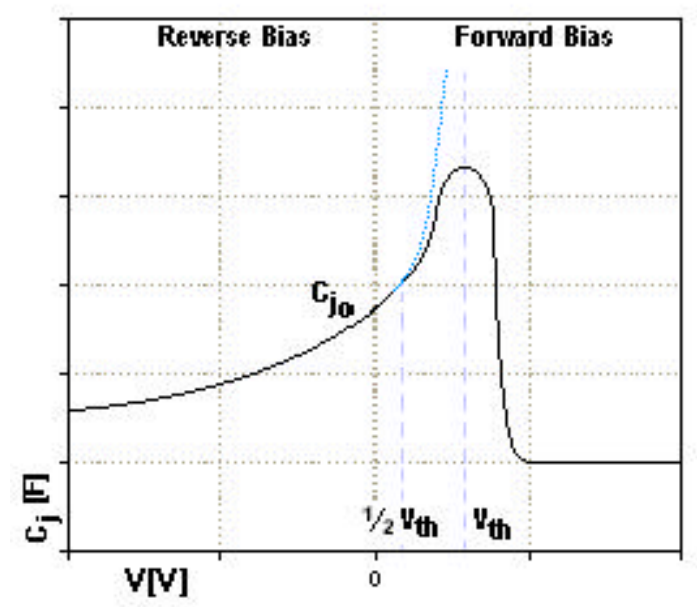

Figure 2.10: C-V plot of a typical semiconductor junction. 
The slope of the curve can be extracted from the $1 / \mathrm{C}_{\mathrm{j}}^{2}$ plot, this slope can be understood as the derivative of $1 / \mathrm{C}_{\mathrm{j}}^{2}$ in respect to the applied voltage. Equation 2.19 shows how the carrier concentration can be extracted using this analysis.

$$
\begin{gathered}
\frac{1}{C_{j}{ }^{2}}=\frac{2\left(V_{b i}-V-\frac{k_{B} T}{q}\right)}{A^{2} q \varepsilon_{S} N} \\
N=\frac{-2}{A^{2} q \varepsilon_{S}[\text { slope }]} \\
{[\text { slope }]=\frac{\partial\left(\frac{1}{C_{j}^{2}}\right)}{\partial V}}
\end{gathered}
$$

Another piece of information that can be extracted from this analysis is the built-in voltage. One can find an expression for the built-in voltage by manipulating equation $2.18 \mathrm{a}$ at a zero bias. This result is given by equation 2.20a.

$$
V_{b i}=\frac{A^{2} q \varepsilon_{S} N}{2 C_{j_{0}}{ }^{2}}+\frac{k_{B} T}{2 q}
$$

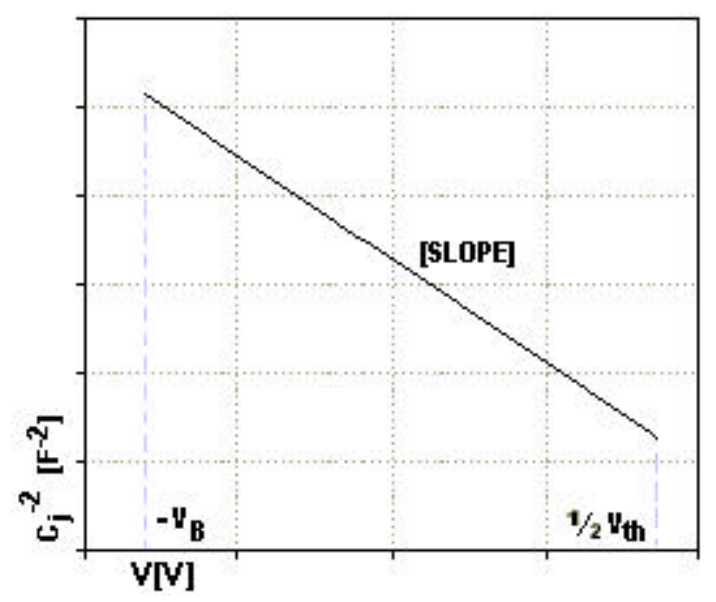

Figure 2.11: Plot of $1 / \mathrm{C}_{\mathrm{j}}^{2}$ versus voltage. 
Applying the result of equation $2.19 \mathrm{~b}$ into equation $2.20 \mathrm{a}$ one finds a more convenient form of expressing the built-in voltage. Equation 2.20b implies that it can be found in a simple

manner. First the ratio of the intercept over the slope is found directly from the plot of $\left(1 / \mathrm{C}_{\mathrm{j}}{ }^{2}\right)$ and then this value is subtracted from half of the thermal voltage.

$$
V_{b i}=\frac{k_{B} T}{2 q}-\frac{\left(\frac{1}{C_{j_{0}}^{2}}\right)}{[\text { slope }]}
$$

All the analysis made up to this point consider a set of ideal conditions in the junction. The first assumption made was that the geometry of the depletion layer is of a parallel plate capacitor, and the voltage drop varies linearly across it. If the shape of the depletion layer is changed, or if the voltage drop varies in a different way, the Poisson equation and the Gauss equation have to be recalculated in order to find more appropriate expressions. Another assumption was that the model was entirely derived assuming that the capacitance and the resistance of the junction are frequency-independent. Also, the model derived assumes that the junction is free of interface-trapped charges, and the resistance of the contact is negligible compared to the resistance of the junction.

An extension of $\mathrm{C}-\mathrm{V}$ analysis is the deep level transient spectroscopy, or DLTS. A brief discussion of this technique is presented in Appendix E.

\subsubsection{EQUIV ALENT ELECTRICAL CIRCUIT MODELING}

Up to this point, the models used were ideal. In this section, some additions are presented in order to find a more realistic model of a depletion layer in a junction. The first addition is the presence of the resistance of the contact. A model that describes the depletion region in a semiconductor taking into account the presence of this series resistance is shown in figure 2.12 [8]. In this model, (r) is the series resistance, which is found in series with the parallel of the junction capacitance $(\mathrm{C})$ and the conductance of the junction $(\mathrm{G})[8]$. 


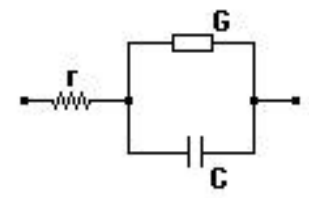

Figure 2.12: Circuit model of a depletion region.

The input impedance of this model is given by equation 2.21a. Manipulating this equation, one finds another form for the input impedance given by equation $2.21 \mathrm{~b}$.

$$
\begin{gathered}
Z_{\text {in }}=\frac{1}{G+j \omega C}+r \\
Z_{\text {in }}=\frac{1}{\frac{G(1+r G)+\omega^{2} C^{2} r}{(1+r G)^{2}+(\omega C r)^{2}}+\frac{j \omega C}{(1+r G)^{2}+(\omega C r)^{2}}}
\end{gathered}
$$

The importance of expressing the input impedance as in equation $2.21 \mathrm{~b}$ is that it represents a new circuit with an equivalent conductance in parallel with an equivalent capacitance. This model is shown in figure 2.13. In this new model, the equivalent conductance $\left(\mathrm{G}^{\prime}\right)$ and the equivalent capacitance are given by equations $2.21 \mathrm{c}$ and $2.21 \mathrm{~d}$ respectively.

$$
\begin{gathered}
G^{\prime}=\frac{G(1+r G)+\left(\omega^{2} C^{2} r\right)}{(1+r G)^{2}+(\omega C r)^{2}} \\
C^{\prime}=\frac{C}{(1+r G)^{2}+(\omega C r)^{2}}
\end{gathered}
$$

The purpose of having an equivalent model as shown in figure 2.13 is that most of the commercial $\mathrm{C}-\mathrm{V}$ analyzers can only measure a conductance in parallel with a capacitance. Therefore, when measuring a junction where the series resistance is significant, what one really measures is the circuit modeled by equations $2.21 \mathrm{c}$ and $2.21 \mathrm{~d}$. In these expressions $C^{\prime}$ and $G^{\prime}$ are the capacitance and conductance measured by equipment, whereas $\mathrm{C}$ and $\mathrm{G}$ are the real 
capacitance and conductance of the junction. A plot of the measured capacitance against the test frequency is shown on figure 2.14 .

The importance of figure 2.14 is that a measurement of the real junction capacitance should be performed at low frequencies. Also, analyzing equation $2.21 \mathrm{~d}$, the product $\mathrm{rG}$ should be smaller than the unity. Otherwise, equation $2.21 \mathrm{~d}$ has to be solved to find the junction capacitance at a specific frequency. This resulting expression is given by equation 2.21e.

$$
C=\frac{1-\sqrt{1-4 C^{\prime 2}(\omega r)^{2}(1+r G)^{2}}}{2 C^{\prime}(\omega r)^{2}}
$$

This correction is only necessary when the series resistance has a very high value. In the cases where the product $\mathrm{rG}$ is smaller than the unity, and the frequency is small, equations $2.21 \mathrm{~d}$ and $2.21 \mathrm{e}$ can be reduced to approximately $\mathrm{C}^{\prime}=\mathrm{C}$.

Equation 2.21d also shows that C-V measurements are strongly affected by frequency. Solving equation $2.19 \mathrm{c}$ assuming the measured capacitance given by equation 2.21 , one finds the expression given by equation $2.22 \mathrm{a}$.

$$
\frac{\partial C^{\prime-2}}{\partial V}=-\frac{2 \cdot(1+r G)^{4}}{A^{2} q \varepsilon_{S} N}+\frac{A^{2} q \varepsilon_{S} N(\omega r)^{4}}{2\left(V_{b i}-V-V_{T}\right)^{2}}
$$

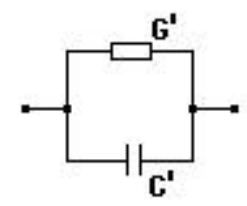

Figure 2.13: Equivalent model of a depletion region. 


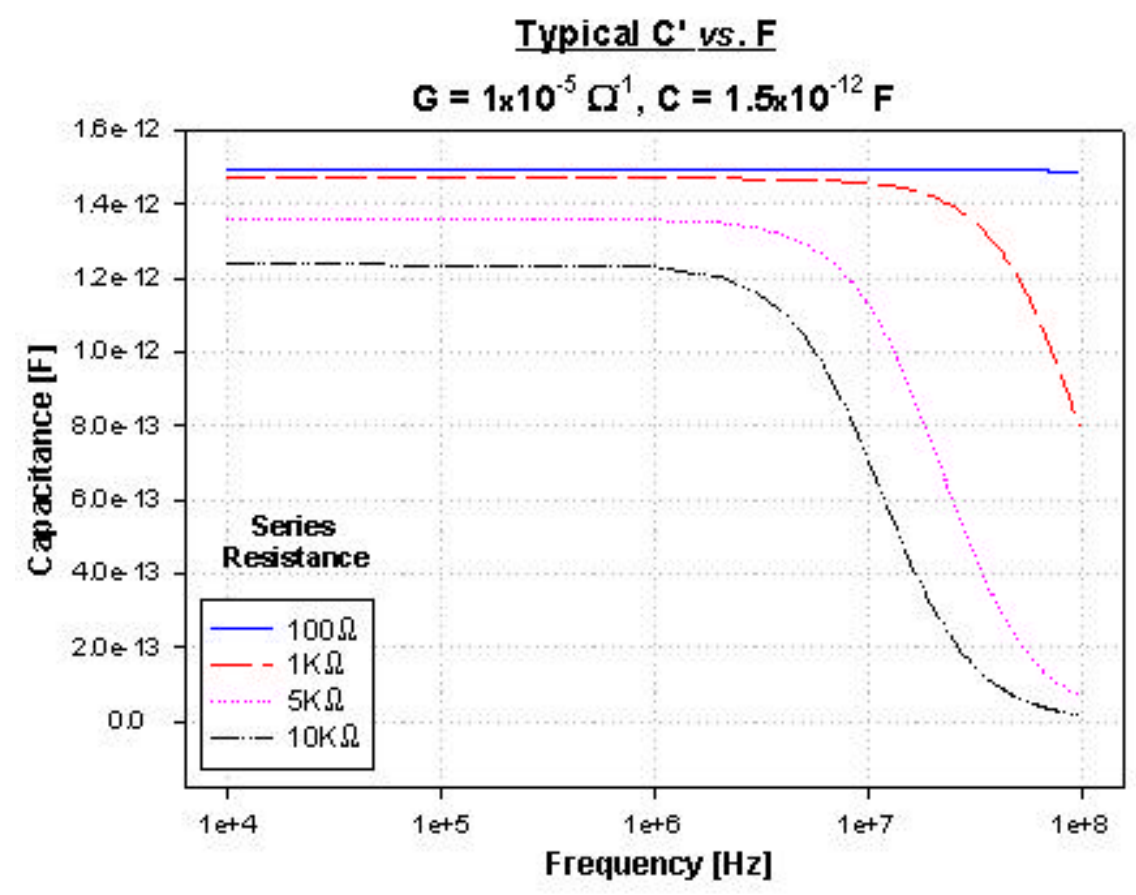

Figure 2.14: Typical plot of capacitance at a specific reverse bias versus frequency of a junction.

Combining equation 2.22a with equation $2.19 \mathrm{~b}$, one finds an expression for the carrier concentration. This last expression is given by equation $2.22 \mathrm{~b}$.

$$
N^{\prime}=-\frac{2}{A^{2} q \varepsilon_{S}} \cdot \frac{1}{-\frac{2(1+r G)^{4}}{A^{2} q \varepsilon_{S} N}+\frac{A^{2} q \varepsilon_{S} N(\omega r)^{4}}{2\left(V_{b i}-V-V_{T}\right)}}
$$

Some important pieces of information can be extracted from equation $2.22 \mathrm{~b}$. The first is that the effective measured carrier concentration decreases as the test frequency is increased. Also, the real carrier concentration can be extracted from equation $2.22 \mathrm{~b}$ when the test frequency is made small. The resulting expression is given by equation $2.22 \mathrm{c}$.

$$
N^{\prime}=\frac{N}{(1+r G)^{4}}
$$


If the product of the series resistance and the junction AC conductivity is made less than the unity, equation $2.22 \mathrm{c}$ reduces to the simple case where the measured carrier concentration tends to the real carrier concentration. A plot of the carrier concentration versus frequency is shown on figure 2.15 .

Based on equations $2.22 \mathrm{~b}$ and $2.22 \mathrm{c}$, in order to measure the carrier concentration of a material, one should take the measurement at low frequencies and have a product $\mathrm{rG}$ much smaller than the unity. Otherwise, equation $2.22 \mathrm{~b}$ has to be solved for the real carrier concentration at a specific frequency.

Another consequence of the presence of a series resistance is the frequency dependence of the built-in voltage. Combining equations 2.20a and 2.22b, one finds an expression for the measured built-in voltage. This expression is given by equation $2.23 \mathrm{a}$.

$$
V_{b i}{ }^{\prime}=\frac{-A^{2} q \varepsilon_{S} N^{2}\left(V_{b i}-V_{T}\right) C_{j 0}{ }^{-1}}{-4\left(V_{b i}-V_{T}\right)(1+r G)^{4}+A^{2} q^{2} \varepsilon_{S}{ }^{2} N^{2}(\omega r)^{4}}+V_{T}
$$

Making the test frequency tend to very small values, equation $2.23 \mathrm{a}$ reduces to equation 2.23b.

$$
V_{b i}^{\prime}=\frac{V_{b i}}{(1+r G)^{4}}
$$

If once again the product $\mathrm{rG}$ is made smaller than the unity, equation $2.23 \mathrm{~b}$ reduces to the case where the measured built-in voltage tends to the real built-in voltage of the junction.

\subsubsection{INTERFACE TRAPPED CHARGES}

Another addition to the depletion region model is the inclusion of interface-trapped charges (surface states). Shockley, among others, studied the existence of surface states occupying the forbidden band up to the Fermi level in semiconductors. In this study, Shockley shows that this occupancy of states in the forbidden band is due to an interruption in the 
periodicity of the lattice of the semiconductor due to these surface states [9]. On clean surfaces of silicon, charge concentrations as high as the density of the surface atoms were found [9]. However, these charges could be completely neutralized by hydrogen annealing at $450^{\circ} \mathrm{C}$ for a short period of time. The occupancy of these surface states is free to move up and down within the forbidden band as the applied voltage is changed. The Fermi level, on the other hand, remains unchanged. At a specific applied voltage the occupancy of the surface states can be able to cross the Fermi level, causing a change in charge in the interface trap [6]. This variation of charges at an applied voltage causes an equivalent capacitance to appear.

A more accurate model for a depletion region can be constructed taking this behavior into consideration. Assuming, in the previous model (Fig. 2.12), that the conductance tends to zero, and the series resistance is negligible, the interface-trapped charges can be incorporated to the model as shown in figure 2.16 .

\section{Typical Carrier Conc. vs. F}

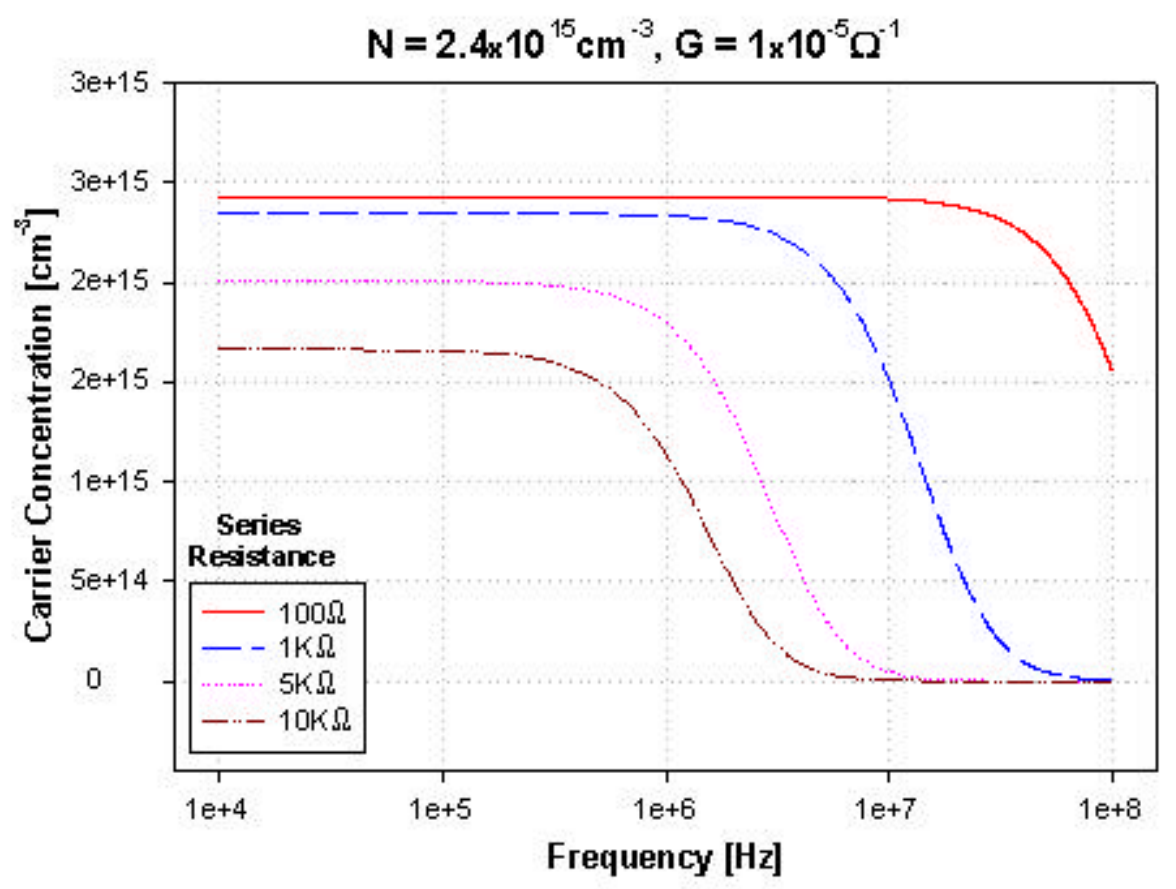

Figure 2.15: Typical plot of carrier concentration versus frequency of a junction. 


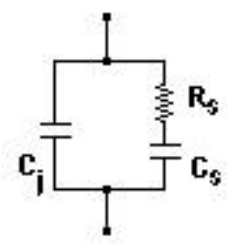

Figure 2.16: Circuit model of a depletion region including surface states, and neglecting the series resistance and the junction conductance.

In this model, $\mathrm{R}_{\mathrm{S}}$ and $\mathrm{C}_{S}$ are the resistance and capacitance associated with the interfacetrapped charges, such that their product $\mathrm{R}_{S} \mathrm{C}_{S}$ is defined as the interface-trap lifetime $(\tau)$. The input impedance of this model is given by equation $2.24 \mathrm{a}$, which can be rearranged in a simpler form given by equation $2.24 \mathrm{~b}$.

$$
\begin{gathered}
Z_{i n}=\frac{1}{j \omega C_{j}+\frac{1}{\frac{1}{j \omega C_{S}}+R_{S}}} \\
Z_{i n}=\frac{1}{\frac{\omega^{2} \tau C_{S}}{1+\omega^{2} \tau^{2}}+j \omega\left(C_{j}+\frac{C_{S}}{1+\omega^{2} \tau^{2}}\right)}
\end{gathered}
$$

This last equation is the parallel of an equivalent conductance with an equivalent capacitance. Their expressions are given by equations $2.25 \mathrm{c}$ and $2.25 \mathrm{~d}$ respectively.

$$
\begin{gathered}
G^{\prime}=\frac{\omega^{2} \tau C_{S}}{1+\omega^{2} \tau^{2}} \\
C^{\prime}=\left(C_{j}+\frac{C_{S}}{1+\omega^{2} \tau^{2}}\right)
\end{gathered}
$$

As explained in the previous session, commercial capacitance meters measure the capacitance shown in equation $2.25 \mathrm{~d}$. This equation has to be solved for $\mathrm{G}$ in order to find the value of the real junction capacitance. 
This model implies that, at the presence of surface states, a high frequency measurement has to be performed in order to eliminate the influence of these states in the computed capacitance. At sufficiently high frequencies, equation $2.21 \mathrm{~d}$ reduces to approximately the junction capacitance, whereas at low frequencies, the $\mathrm{CV}$ curve is shifted due to the presence of surface states. Physically, this implies that at high frequencies, the interface-trapped charges are unable to follow the $\mathrm{AC}$ signal, and therefore do not contribute significantly to the total measured capacitance. This behavior can be visualized in figure 2.17. Consequently, verifying a shift of the capacitance $v s$. voltage curve at several frequencies is a possible test to identify the presence of interface-trapped charges.

As seen on figure 2.17 the effect of interface-trapped charges on the built-in voltage is strong, since more charges are required to generate a specific surface potential. Therefore a high frequency $\mathrm{C}-\mathrm{V}$ measurement is favored when one is concerned about the built-in voltage.

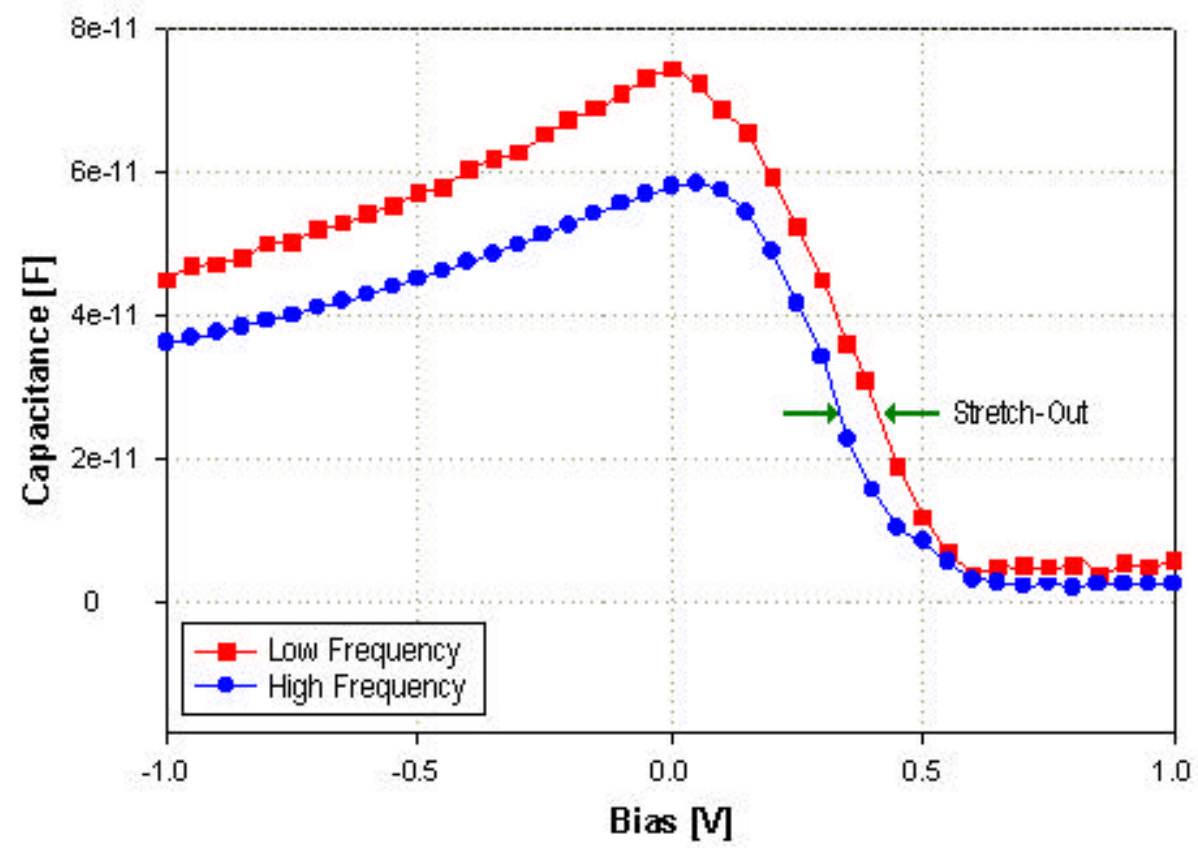

Figure 2.17: Stretch-out in the C-V curve due to interface-trapped charges. 
In summary, interface-trapped charges impose a lower limit in the frequency at which the measurement is performed, whereas the effect of a series resistance, as shown in the previous section, imposes an upper limit to this frequency. Correction factors can be used to adjust the measured values, as shown by equation 2.21e. Also, these nonideallities can be minimized if:

i) The product of the series resistance and the junction conductance is much smaller than the unity, and the measurement of the capacitance is taken at very low frequencies; this way, the effects of the series resistance are minimized;

ii) The measurement of the capacitance is taken at a sufficient high frequency so that the effects of the interface-trapped charges are minimized.

In conclusion, the measurement of the capacitance of a junction presents a trade-off with respect to the probing frequency and experimental curves have to be measured in order to find the optimal measuring frequency.

\subsubsection{LOSS TANGENT}

One last figure of merit that has to be introduced is the loss tangent or dissipation factor. Making a phase analysis of the model described by junction conductance in parallel with a junction capacitance, one finds the diagram shown in figure 2.18. In this figure, $\mathrm{b}$ is the current that flows through the junction, $G$ is the conductance of the junction, ? is the measuring frequency, $\mathrm{C}$ is the capacitance of the junction, $\mathrm{X}$ is the total impedance of the junction, and $\theta$ is the angle between the imaginary and the real parts of the junction impedance.

The loss tangent is defined as the tangent of the angle $\theta$. This tangent is also equal to the imaginary over the real portion of the junction impedance. This relation is given by equation 2.26 . 


$$
D=\tan \theta=\frac{\frac{1}{\omega c}}{\frac{1}{G}}=\frac{G}{\omega C}
$$

The dissipation factor also says how good the junction capacitance is. In other words, in an ideal case, one would expect to have all the current $I_{0}$ to flow through the junction capacitance so that most of the previous formulations would be closer to the ideal case. However, the presence of a parallel junction conductance drains part of the current and makes the junction capacitance show a leaky behavior. This leaky behavior, in turn, can be found by the dissipation factor that says how big the reactance of the junction is compared to its conductance. Good values of the dissipation factor are usually smaller than 0.1 and are responsible for changes in accuracy of commercial capacitance meters.

Another point to be evaluated is how the dissipation factor varies when the measured conductance and capacitance are frequency-dependent. This can be analyzed by combining equations $2.21 \mathrm{c}, 2.21 \mathrm{~d}$, and 2.26. The resulting expression is given by equation 2.27.

$$
D=\frac{G(1+r G)+\left(\omega^{2} C^{2} r\right)}{\omega C}
$$

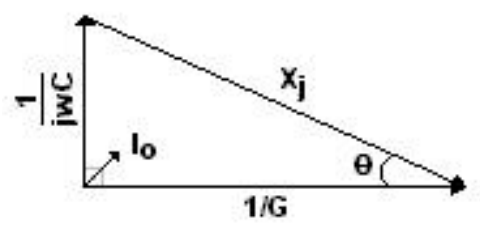

Figure 2.18: Phase diagram of an ideal depletion layer. 
A typical plot of equation 2.27 is given by figure 2.19. This plot shows that as frequency increases, the dissipation factor decreases until a certain point of minimum. After this point, the loss starts to increase back again. Any measurement of the capacitance, therefore, should take this characteristic into consideration. To avoid any misreading in the measurement of capacitance, points of minimum of the dissipation factor should be chosen to guarantee the lowest losses possible.

\subsubsection{DIFFUSION CAPACITANCE}

The diffusion capacitance is an additional capacitance that appears in a depletion region when a small AC signal is superposed onto a DC signal. In order to find he expression for this capacitance, one can model this superposed signal as shown in equations 2.28a, and 2.28b.

$$
\begin{aligned}
& V=V_{0}+V_{1} e^{j \omega t} \\
& I=I_{0}+I_{1} e^{j \omega t}
\end{aligned}
$$

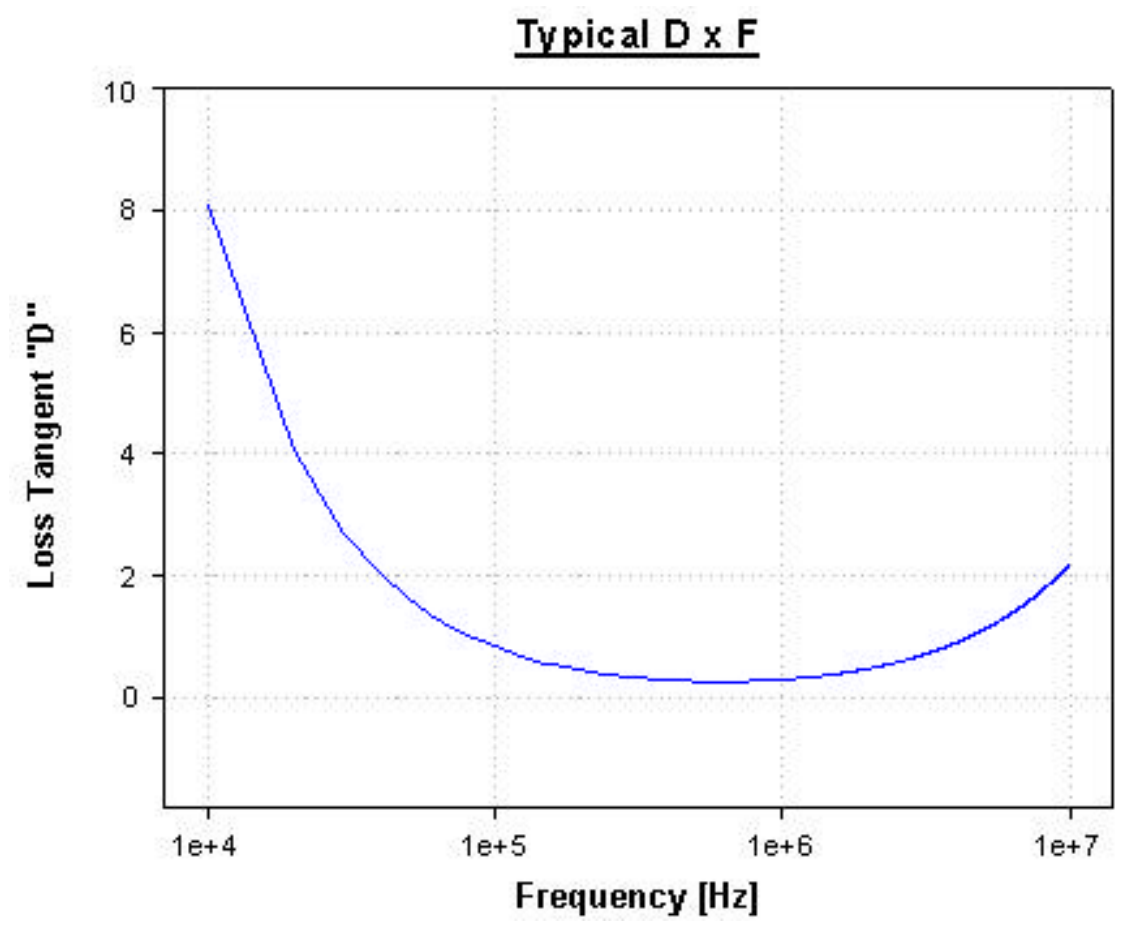

Figure 2.19: Typical plot of loss tangent versus frequency of a junction. 
In these expressions, $\mathrm{V}_{0}$ and $\mathrm{I}_{0}$ are the DC components of the applied voltage and current, whereas $V_{1}$ and $I_{1}$ are the $A C$ components of the applied signal. In a depletion layer, the density of $\mathrm{p}$-type carriers is given by equation 2.29. In this expression, $\mathrm{p}_{\mathrm{t}}$ is the density of $\mathrm{p}$-type carriers in the $n$-side of the junction, $p_{n o}$ is the density of ptype carriers in the 1 side of the junction at equilibrium, and $\mathrm{V}$ is the applied voltage.

$$
p_{n}=p_{n 0} \cdot \exp \left(\frac{q V}{k_{B} T}\right)
$$

Combining equation 2.28a and equation 2.29, one finds an expression for the density of p-type carriers that takes into account a test AC signal. This expression is given by equation 2.30.

$$
p_{n}=p_{n o} \cdot \exp \left(\frac{q V_{0}}{k_{B} T}\right)+\frac{p_{n o} q V_{1}}{k_{B} T} \cdot \exp \left(\frac{q V_{0}}{k_{B} T}\right) \cdot e^{j \omega t}
$$

The same process can be carried out to find an expression for the density of n-type carriers in the pregion. From this equation it is clear that there is a term that depends only on the DC signal and a term that depends on the AC part of the signal. Substituting equation 2.30 in the continuity equation given by expression 2.31a, one finds a more complete expression for the density of carriers. This last formula is given by equation $2.31 \mathrm{~b}$.

$$
\begin{gathered}
\frac{\partial p}{\partial t}=-U_{n}+\frac{1}{q} \nabla \cdot J_{p} \\
\frac{\partial^{2} p_{n}}{\partial x^{2}}-\frac{p_{n}}{\frac{D_{p} \tau_{p}}{1+j \omega \tau_{p}}}=0
\end{gathered}
$$

In these last two equations, $\mathrm{U}_{\mathrm{h}}$ is the electron recombination rate in the p-region, $\mathrm{J}_{\mathrm{p}}$ is the hole current density, $D_{p}$ is the p-type carriers diffusion constant, and $t_{p}$ is the hole lifetime. 
Equation 2.31b assumes a solution if the carrier lifetime can be expressed as shown in equation 2.32 .

$$
\tau_{p}{ }^{\prime}=\frac{\tau_{p}}{1+j \omega \tau_{p}}
$$

Substituting these expressions into the Shockley equation given by equation 2.33a, one finds an expression for the current density that flows through the junction. This formula is given by equation $2.33 b$.

$$
\begin{gathered}
J=\left(\frac{q D_{p} p_{n o}}{L_{p}}+\frac{q D_{n} n_{p o}}{L_{n}}\right) \cdot\left(\exp \left(\frac{q V}{k_{B} T}\right)-1\right) \\
J=\frac{q V_{1}}{k_{B} T}\left(\frac{q D_{p} p_{n o}}{\frac{L_{p}}{\sqrt{1+j \omega \tau_{p}}}+\frac{q D_{n} n_{p o}}{L_{n}}}\right) \cdot \exp \left(\frac{q V_{0}}{k_{B} T}\right)
\end{gathered}
$$

In these last two expressions, $\mathrm{L}$ is the diffusion length, defined as the square root of the diffusion constant times the lifetime of the carrier. Equation $2.33 \mathrm{~b}$ can be manipulated to find an equivalent $\mathrm{AC}$ admittance. This expression is shown in equation 2.34.

$$
Y=\frac{J_{1}}{V_{1}}=G_{d}+j \omega C_{d}
$$

This last equation represents a conductance in parallel with a capacitive reactance. At low frequencies, the diffusion conductance and capacitance are given by equations $2.35 \mathrm{a}$, and $2.35 \mathrm{~b}$.

$$
G_{d 0}=\frac{q}{k_{B} T}\left(\frac{q D_{p} p_{n o}}{L_{p}}+\frac{q D_{n} n_{p o}}{L_{n}}\right) \cdot \exp \left(\frac{q V_{0}}{k_{B} T}\right)\left[\frac{\Omega^{-1}}{c m^{2}}\right]
$$




$$
C_{d 0}=\frac{q}{k_{B} T}\left(\frac{q L_{p} p_{n 0}}{2}+\frac{q L_{n} n_{p 0}}{2}\right) \cdot \exp \left(\frac{q V_{0}}{k_{B} T}\right)\left[\frac{F}{c m^{2}}\right]
$$

Equations $2.35 \mathrm{a}$, and $2.35 \mathrm{~b}$ are also often found in the literature as the equations of diffusion conductance and capacitance [6]. One important thing that is noticed from these equations is that the capacitance and the admittance increase exponentially as the DC voltage is increased. Furthermore, these two elements are found in parallel with the standard conduction and capacitance of the depletion layer, as shown in figure 2.20. Therefore, the total capacitance is the depletion capacitance added to the diffusion capacitance, and the total conductance is the depletion conductance added to the diffusion conductance.

This may lead to an inappropriate reading of the capacitance for DC values where the current that flows through the diode starts to be dictated by the Shockley equation. Also, the admittance starts to increase exponentially. This causes the previous models to have high losses, and the dissipation factor limits any reading of the junction capacitance.

Another point that has to be observed is how the AC models of the diffusion conductance and capacitance vary with frequency. The expressions that govern their frequency-dependent model are somewhat difficult to derive, and a plot is usually the best way to visualize their behaviors. This plot of the diffusion capacitance and conductance versus frequency is shown on figure $2.21[6]$.

In conclusion, the mathematical model used to calculate the expression of a junction capacitance consists of several nonideallities. Most of them can be modeled using an electrical circuit analysis, and in many cases can be minimized if certain conditions are obeyed. A model that incorporates all the imperfections seen in this chapter is shown on figure 2.22. Some of the conditions were already stated in session 2.3.1.1 such as having a small series resistance and using a relative high frequency to avoid the effects of interface-trapped charges. 


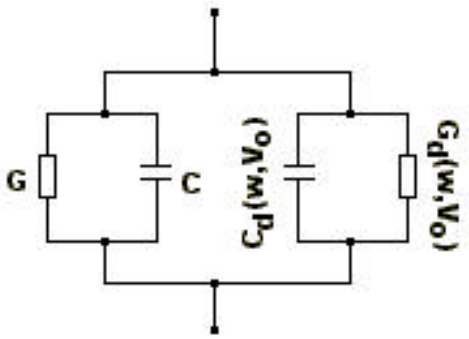

Figure 2.20: Electrical circuit model of a depletion layer including a diffusion capacitance and conductance.

Analyzing the model shown on figure 2.22, the diffusion capacitance and conductance should be small compared to the depletion capacitance and conductance. The last point to be analyzed is the dissipation factor that, as stated in the previous session, should be the minimum achievable and preferably smaller than 0.1 . Table 2.1 gives a chart illustrating the points that have to be observed in any measurement related to the depletion region.

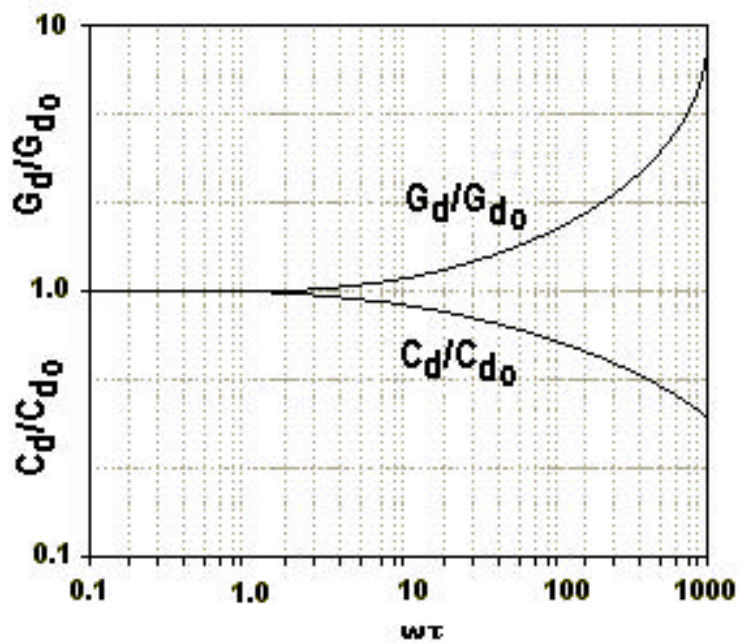

Figure 2.21: Diffusion capacitance and conductance as functions of frequency. 


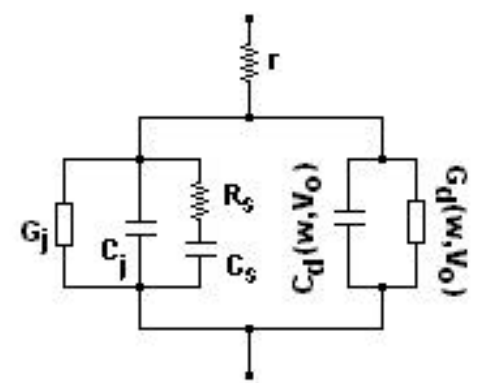

Figure 2.22: Total electrical circuit model of a depletion region.

Table 2.1: Points to be observed during measurements related to a depletion region.

\begin{tabular}{|l|l|}
\hline \multicolumn{2}{|c|}{ POINTS TO OBSERVE } \\
\hline Series Resistance: & $\begin{array}{l}\text { Try to have product rG much smaller than the unity; } \\
\text { Choose low frequencies and a point in the flat region of the } \\
\text { frequency curve to avoid the frequency effects of the series } \\
\text { resistance model; } \\
\text { Minimum of dissipation factor gives the point of lowest losses. }\end{array}$ \\
\hline Interface States: & $\begin{array}{l}\text { Choose high frequency so that the states cannot follow the test } \\
\text { signal. }\end{array}$ \\
\hline & $\begin{array}{l}\text { High frequency and large negative bias to avoid its additional } \\
\text { influence. }\end{array}$ \\
\hline Diffusion Cap.:
\end{tabular}

\subsection{CURRENT VS. VOLTAGE ANALYSIS (I-V) ON PLANAR CIRCULAR STRUCTURES}

As shown before, in order to perform $\mathrm{C}-\mathrm{V}$ measurements, some other important characteristics such as the breakdown voltage, threshold voltage, and the series resistance have to be known beforehand. This information can be extracted by plotting a current versus voltage (IV) curve of the device under analysis. Some of these points were already discussed, however, the sheet resistance and the specific contact resistance require further attention.

When FV measurements are performed on contact structures, the geometry of the contact has to be taken into account. Basically two kinds of geometries can be used: rectangular and circular. The former has the advantage of simplicity, however it has the disadvantage of leakage 
currents flowing at the edges of the contact. In order to eliminate these spurious currents, one can either create a mesa structure to confine the current within the contacts, or use a circular geometry.

A typical circular geometry for contacts is shown in figure 2.23. If this geometry is used, and a current flows through it, then the voltage drop between its contacts (dot and ring) is given by equation 2.36 [11].

$$
\Delta V=\frac{i_{0} R_{S}}{2 \pi}\left[\ln \left(\frac{r_{1}}{r_{0}}\right)+\frac{L_{T}}{r_{0}} \frac{I_{0}\left(\frac{r_{0}}{L_{T}}\right)}{I_{1}\left(\frac{r_{0}}{L_{T}}\right)}+\frac{L_{T}}{r_{1}} \frac{K_{0}\left(\frac{r_{1}}{L_{T}}\right)}{K_{1}\left(\frac{r_{1}}{L_{T}}\right)}\right]
$$

In equation 2.36, $\mathrm{i}_{0}$ is the current that flows through the contacts, $\mathrm{R}_{\mathrm{S}}$ is the sheet resistance of the conducting layer of the semiconductor, $r_{0}$ and $r_{1}$ are the radii of the dot and the ring as shown in figure $2.23, \mathrm{~b}, \mathrm{I}_{1}$ and $\mathrm{K}_{0}, \mathrm{~K}_{1}$ are the modified Bessel functions of zero and first order, and $\mathrm{L}_{\mathrm{T}}$ is a constant defined as in equation 2.37. Typical values for $\mathrm{L}_{\mathrm{T}}$ are between a few microns and hundreds of microns.

$$
L_{T}=\sqrt{\frac{R_{C}}{R_{S}}}
$$

In this last equation, $R_{C}$ is the specific contact resistance. If the radii $r_{0}$ and $r_{1}$ are at least four times bigger than the constant $\mathrm{L}_{\mathrm{T}}$, the Bessel functions can be approximated by unity [12]. Considering these approximations, equation 2.36 can be rewritten as:

$$
\Delta V=\frac{i_{0} R_{S}}{2 \pi}\left[\ln \left(\frac{r_{1}}{r_{1}-d}\right)+L_{T}\left(\frac{1}{r_{1}}+\frac{1}{r_{1}-d}\right)\right]
$$

A plot of equation 2.38 as a function of the separation between the dot and the ring is shown in figure 2.24 . 


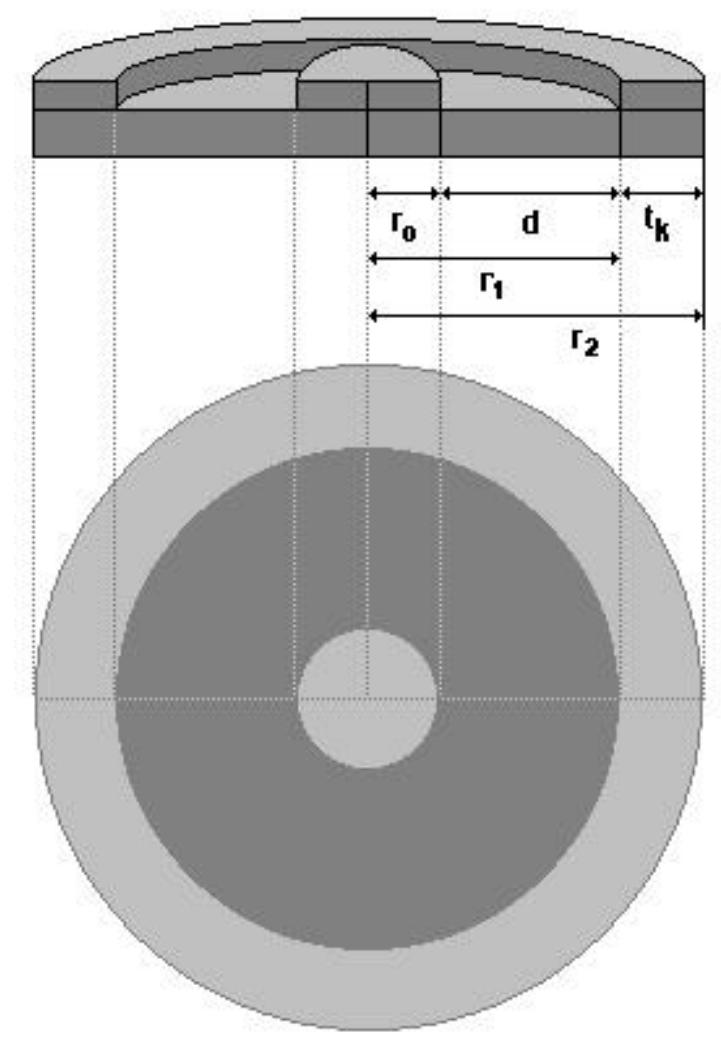

Figure 2.23: Circular contact geometry.

Based on equation 2.38 one can use a least square fitting routine to obtain the sheet resistance and the constant $\mathrm{L}_{\mathrm{T}}$. Having a value for the constant $\mathrm{L}_{\mathrm{T}}$ and the sheet resistance one can easily find the contact resistance by using equation 2.37 .

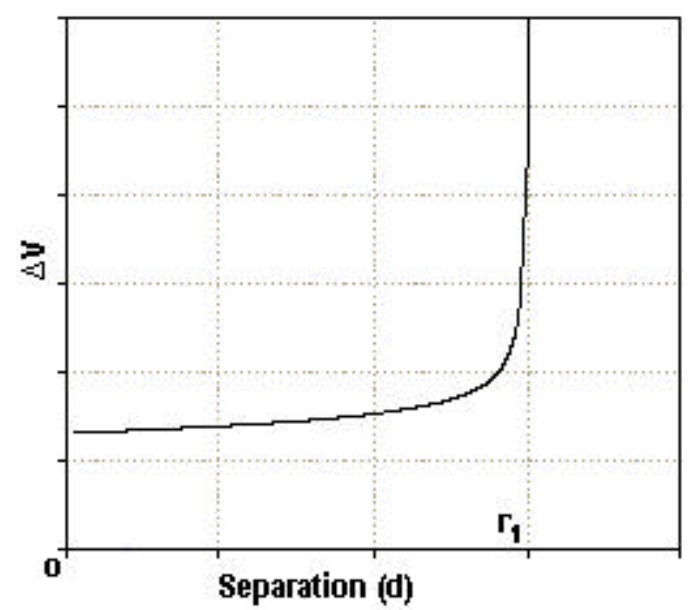

Figure 2.24: The voltage drop between contacts as a function of their separation. 


\section{Chapter 3 - Experimental Procedures}

\subsection{MASKS DESIG N}

The first step in the creation of contacts to a specific material is the design of the mask that contains the structures that compose the contacts. The mask works as an image of the structures that are transferred to a photoresist and later delineated on a metal. For the current study the masks used consisted of two layers, one for Ohmic contacts, and a second for Schottky contacts. Therefore, beyond the contact structures, the masks also contained alignment structures. Furthermore, since the gallium nitride samples in this study did not have a conductive backside, circular coplanar structures were chosen to create the contacts.

The first mask design was based on the circular geometry proposed by several authors $[11][12][13][18][19]$. This mask was composed of two layers. One layer contained dots of several radii, ranging from $140 \mu \mathrm{m}$ to $210 \mu \mathrm{m}$ [17][19]. Also on the first layer there were dots with the same radii surrounded by rings of $250 \mu \mathrm{m}$ of radius, four sets of concentric rings with radii of 100, 120, 180, 250 and 330 $\mathrm{m}$, and four Van der Pauw structures. Layer two only contains rings with $250 \mu \mathrm{m}$ of radius and $100 \mu \mathrm{m}$ of thickness. The second layer can be used for a second metal and thus to create Ohmic and Schottky contacts. This mask layout is shown on figure 3.1 .

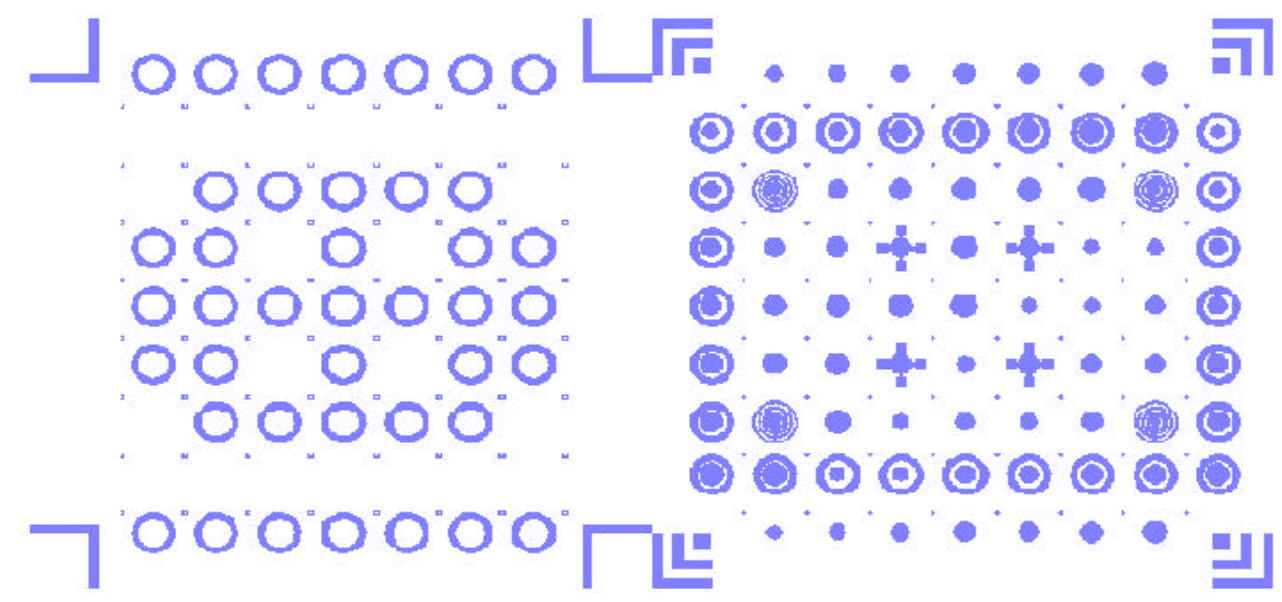

Figure 3.1: First mask design. 
The first mask layout presented a problem. Although there were appropriate structures to create Ohmic and Schottky contacts on it, if one used the dots as Schottky contacts, then the ring-dot structures that were supposed to be Ohmic also become Schottky. Also, Schottky contacts with different areas showed a strange behavior in $\mathrm{C}-\mathrm{V}$ measurements. This phenomenon will be discussed further in the next chapters.

In order to compensate for the problems of the first mask and have more structures to test the phenomenon of the area of the Schottky contact, a second mask was devised. This second mask also consisted of two layers. The first layer contained rings with different sizes with the same thickness $(100 \mu \mathrm{m})$. Also, on the first layer there were two sets of dot-ring structures to create Ohmic contacts. In these dot-ring structures, all the rings were $100 \mu \mathrm{m}$ thick and separated from the dot by $40 \mu \mathrm{m}$. The inner dots in these dot-ring structures had radii of 50, 100, 150, 200, and $250 \mu \mathrm{m}$.

The second layer only contained dots of different radii that could be use to create Schottky contacts. When both layers are combined, a pattern is created. From top to bottom, the separations between the ring and the dot are 20,50,60, 80, and $100 \mu \mathrm{m}$ respectively. Also, from left to right, the radii of the dots are $250,200,150,100$, and $50 \mu \mathrm{m}$ respectively. This mask design is shown on figure 3.2. There are in both masks identifier texts to show the radii of the structures, as well as alignment marks for coarse and fine alignments.

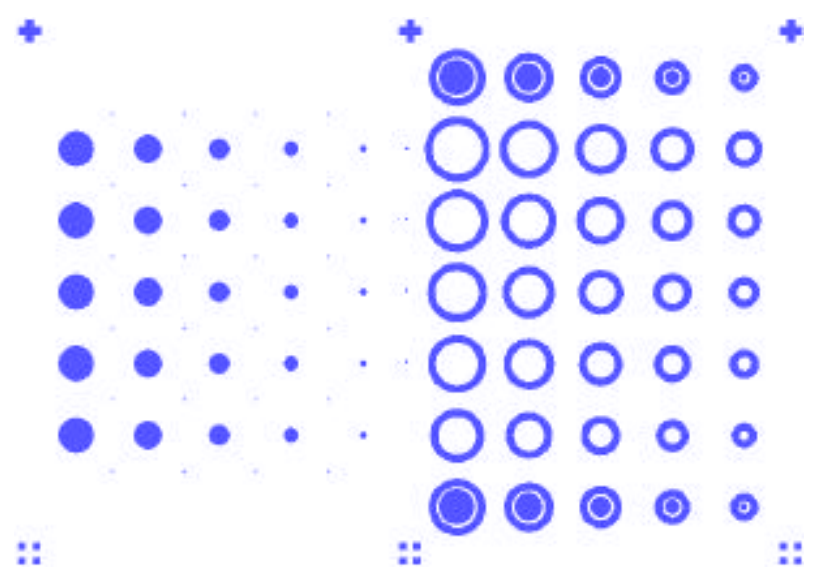

Figure 3.2: Second mask layout. 


\subsection{PREPARATION OF SI SAM PLES}

With the masks designed, the image of the contact structures needs to be transferred to the material under study. This is done following a certain procedure that consists of a set of steps necessary to clean its surface and create the appropriate contacts on it. A flow diagram of these steps is shown in figure 3.3. As seen on this diagram, the sequence starts with a basic cleaning followed by a photoresist patterning and metallization. A lift-off step is necessary to delineate the desired structures on the deposited metal, and a solvent cleaning is performed to prepare the sample for a second metallization. If a double metallization is necessary, the steps following photoresist patterning are repeated.
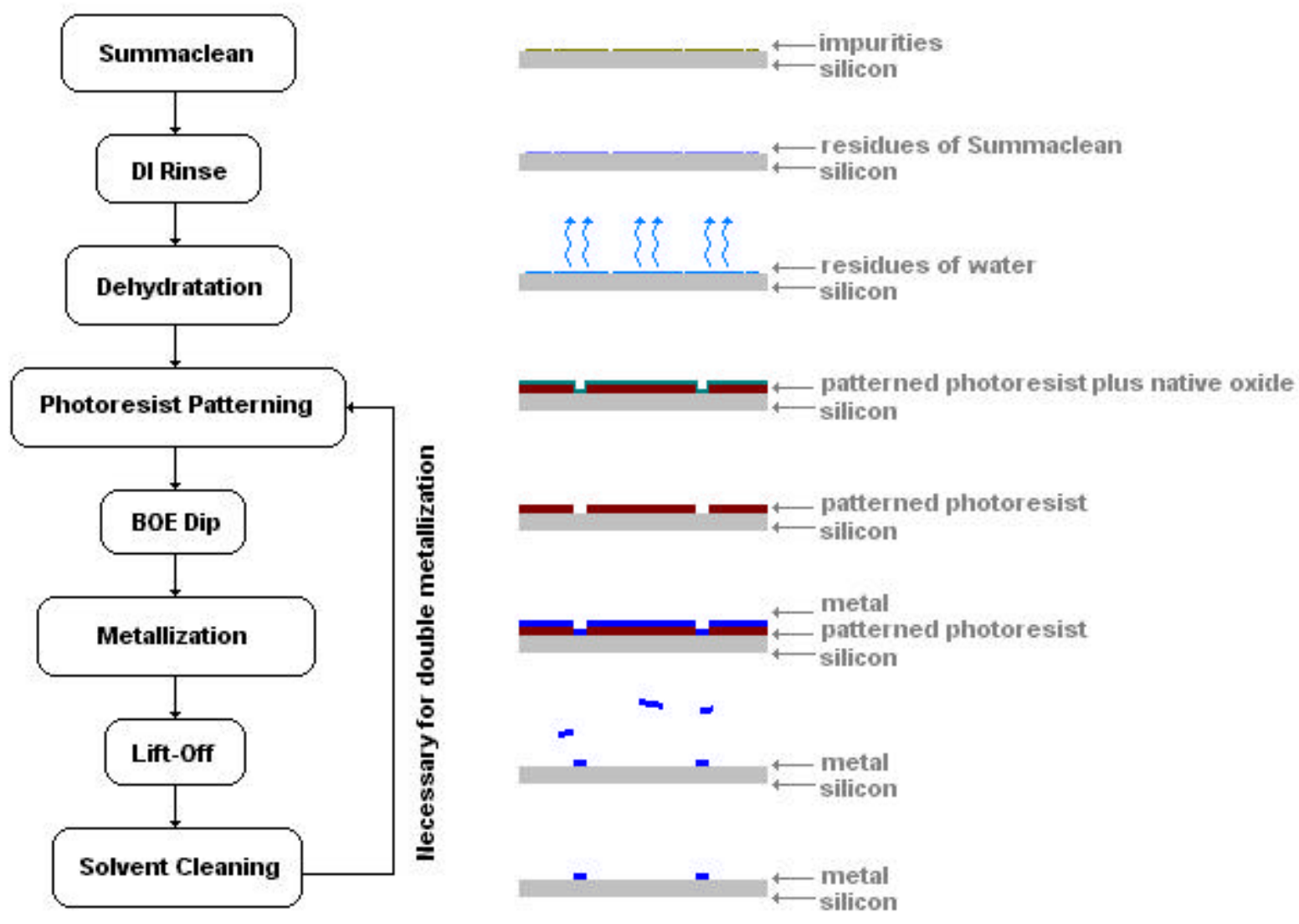

Figure 3.3: Flow diagram of preparation of silicon samples. 
The first step in processing a piece of silicon is to clean it. The standard chemical used to clean silicon is Summaclean [20]. In order to clean the wafer of silicon, a beaker is filled with summaclean and heated in a hot plate up to a temperature between $50^{\circ} \mathrm{C}$ and $60^{\circ} \mathrm{C}$. This is a Corning explosion proof PC-35 hot plate located in the acid hood. The temperature of the solution is measured by stirring the solution with a thermometer, dipping the thermometer down to the middle of the beaker, and waiting for a few seconds until the thermometer reaches thermal equilibrium with the solution. The thermometer never touches the bottom of the beaker to avoid misreading. When the temperature reaches the specified range, the sample is immersed for 30 minutes into the solution using a pyrex holder.

After the piece of silicon is cleaned with Summaclean it is necessary to do a cleaning with de-ionized (DI) water to remove the previous chemical from the surface of the silicon piece. The DI water should have a resistivity higher than $18 \mathrm{M} \Omega-\mathrm{cm}$ and the rinsing is performed in a new beaker with flowing DI water for 5 minutes.

In order to remove the water from the surface of the silicon piece, first it is blown dry with a nitrogen gun. Then, the sample is placed on a hotplate previously heated at $110^{\circ} \mathrm{C}$ for one minute. This is a Thermolyne 560G explosion proof hot plate located in the solvent hood. This step is performed to make sure that the surface of the sample is free of water.

A summary of the cleaning used for silicon samples is shown on table 3.1.

Table 3.1: Preparation process of silicon samples.

\begin{tabular}{|c|c|c|c|}
\hline Step & Chemical & Condition & Duration \\
\hline I & Summaclean & Between $50^{\circ}$ and $60^{\circ} \mathrm{C}$ & $5 \mathrm{~min}$ \\
\hline II & DI Rinse & Rinse & $5 \mathrm{~min}$ \\
\hline III & Dehydratation & Hot Plate at $110^{\circ} \mathrm{C}$ & $1 \mathrm{~min}$ \\
\hline IV & \multicolumn{3}{|c|}{ Photoresist Patterning } \\
\hline V & Diluted BOE & Room temperature & $30 \mathrm{sec}$ \\
\hline
\end{tabular}


Once the sample is dry, it has to be coated with photoresist. First, the wafer is placed on the spinner and vacuum is generated to hold the wafer to the spinner. Then, the photoresist AZ5214 is loaded into a new pipette and it is dropped on the center of the wafer until two thirds of its surface is coated. A Solitec Controller is used to spin the wafer.

The spread time is set to 2 seconds, the spinning time is set to 30 seconds, the ramp settings are 038 and 132, and the spindle speed is $3.95 \mathrm{Krpm}$ (dial shows 473). With these values set on the controller, the wafer spins to create a 1-micron thick film of photoresist on the surface of the sample.

After the film of photoresist coats the silicon pieces, a pre-baking is necessary to promote the adhesion of the photoresist to the pieces. Also, it removes gases from the film and prevents outgassing during the sputtering or evaporation in a high-vacuum chamber. This pre-baking is performed on a previously heated hotplate at $100^{\circ} \mathrm{C}$ for one minute. This is a Thermolyne 560G explosion proof hot plate located in the photoresist hood.

After the pre-baking, the patterns are transferred to the sample. This is done in an M1 Infrared Mask Aligner using an Optical Associates Inc. Intensity Control System Model 783. The lamp used in the aligner is a deep UV mercury-xenon lamp with a wavelength filter of $365 \mathrm{~nm}$ that produces power density of $12 \mathrm{~mW} / \mathrm{cm}^{2}$.

The mask, which is chrome on soda lime, is aligned with the sample and the pressure is increased until the display indicates $3 \mathrm{Lb} / \mathrm{in}^{2}$ between the sample and the mask. Then, the sample is exposed to ultraviolet light. In the case of the 5214 [23] photoresist and using a soda lime chrome mask, the optimal exposure time found was 8 seconds. This first exposure transfers the normal positive image to the photoresist.

The next step for this photoresist is the post-baking. This step is necessary to change the photoresist into the image reversal configuration. The post-baking lasts 45 seconds on a hotplate previously heated to $100^{\circ} \mathrm{C}$. This is the Thermolyne hot plate located in the acid hood. 
A flood exposure is then performed. The flood exposure is a maskless exposure of the sample coated with photoresist to UV. This exposure increases the contrast of the images on the photoresist. This last exposure was determined to be optimal at 45 seconds for the 5214 photoresist on the same aligner.

Once the sample has been flood exposed, it can be developed. The recommended solution for development of this photoresist is 1.2 units of water for 1 unit of AZ300K developer. The development is performed holding the wafer with tweezers and stirring it in the solution until the clear field is completely exposed. This usually takes 30 seconds. However, the development duration is determined by inspecting the clear field of the sample being developed. After the clear fields are completely visible, the wafer is stirred in DI water for one minute to remove the developer from its surface.

After the patterns are developed, the wafer is blown dry with a nitrogen gun. Then, the wafer is dipped into a solution of hydrofluoric acid (100:1 DI water:HF) for 30 seconds to remove the native oxide from its surface. Then the sample is rinsed with flowing DI water for 5 minutes. The sample is again blown dry with a nitrogen gun and is immediately loaded into a high vacuum chamber for metallization.

The sputtering station used in the process is a CVC610. Sputtering conditions are different for different applications. Typical sputtering settings used for the contact metallization in this work are shown in shown in table 3.2.

Once the metal is sputtered on the surface of the sample, the excess metal has to be liftedoff. This step is performed in a Branson ultrasonic bath model 1210. The wafer is placed in a beaker full of acetone. Note hat the quantity of acetone has to be enough to avoid saturation of the solution. Then the wafer is ultrasonically agitated in the bath until complete removal of the excess metal. The duration of the lift-off is determined by inspection of the sample. However, the lift-off process is never intermitted to avoid re-deposition of the metal. A typical lift-off time is approximately one minute. 
Table 3.2: Typical sputtering conditions.

\begin{tabular}{|c|c|}
\hline Chamber pressure & $<9 \times 10^{-7}$ Torr \\
\hline Argon pressure & $\sim 6 \mathrm{mTorr}$ \\
\hline Argon flow & $\sim 88 \mathrm{sccm}$ \\
\hline Power (set) & $0.1 \mathrm{KW}$ \\
\hline Voltage (measured) & $\sim 460 \mathrm{~V}$ for Pt \\
& $\sim 515 \mathrm{~V}$ for $\mathrm{Al}$ \\
& $\sim 430 \mathrm{~V}$ for $\mathrm{Cr}$ \\
\hline Deposition time & 2 minutes cleaning (4 minutes if the target \\
& has been changed) and 5 minutes \\
& deposition. \\
\hline
\end{tabular}

A degreasing step is performed to prepare the sample for a new metallization. The sample after lift-off is not attached to the wafer any longer, and is placed in a beaker full of acetone. This beaker stays heated at a temperature within the range of $40-50^{\circ} \mathrm{C}$ for five minutes. This is done on an explosion proof hot plate in a metal (fireproof) hood. The sample is blown dry with a nitrogen gun and then placed in another beaker full of methanol. This beaker stays heated at a temperature within the range of $40-50^{\circ} \mathrm{C}$ for 5 minutes and then the sample is blown dry again. The temperature should be maintained constant within the specified range in order to avoid boiling of the substances and further problems.

\subsubsection{METALLIZATION OF SI SAM PLES}

An appropriate choice of metals has to be made in order to create Ohmic and Schottky contacts to silicon. All the silicon wafers used in this work were ptype. For silicon the bandgap is $1.12 \mathrm{eV}$, and the electron affinity is $4.05 \mathrm{eV}$. Applying these values to equation $2.15 \mathrm{~b}$ and solving it for the work function, one finds it to be $5.17 \mathrm{eV}$. A metal having a work function close to this value would produce a good Ohmic contact to silicon. Aluminum was the metal chosen to create the Ohmic contacts. Once the metal was deposited and patterned, it was annealed at $450^{\circ} \mathrm{C}$ for 5 minutes. Specifically in the case of p-type silicon, the annealing step is responsible for creating a linear graded junction between silicon and aluminum, allowing a smooth transition between them and a good Ohmic contact. 
Contrary to Ohmic contacts, when creating Schottky contacts one is interested in creating a reasonable barrier heigth. Solving again equation $2.15 \mathrm{~b}$ for the work function, assuming a barrier height bigger than zero, one finds the work function to be smaller than $5.17 \mathrm{eV}$. The metal chosen was chromium, since its work function is $4.6 \mathrm{eV}$ and it is a common material. The barrier height is then found to be $0.57 \mathrm{eV}$ for this metal.

\subsection{PREPARATION OF G aN SAM PLES}

The preparation of gallium nitride samples, similarly to the preparation of silicon samples, consists of a set of steps necessary to clean its surface and create the appropriate contacts to it. A flow diagram of these steps is shown in figure 3.4. As seen on this diagram, the sequence starts with a basic solvent cleaning followed by an acid dip, a photoresist patterning, and metallization. A lift-off step is necessary to delineate the desired structures on the deposited metal, and a solvent cleaning is performed to prepare the sample for a second metallization. If a double metallization is necessary, the steps following photoresist patterning are repeated.

The first step in processing a sample of gallium nitride is to clean it to remove surface impurities and contaminations that may compromise the quality of the future structures. A solvent cleaning is usually the first step in processing semiconductors. The sample is first dipped in a warm solution of a solvent for a few minutes, and then ultrasonically agitated in another solution of the same solvent for a few more minutes [13][14]. This process is then repeated for each of the solvents in the process. In each step of the solvent cleaning process, the tweezers and holders are cleaned with the solvent of the corresponding step and then blown dry. 


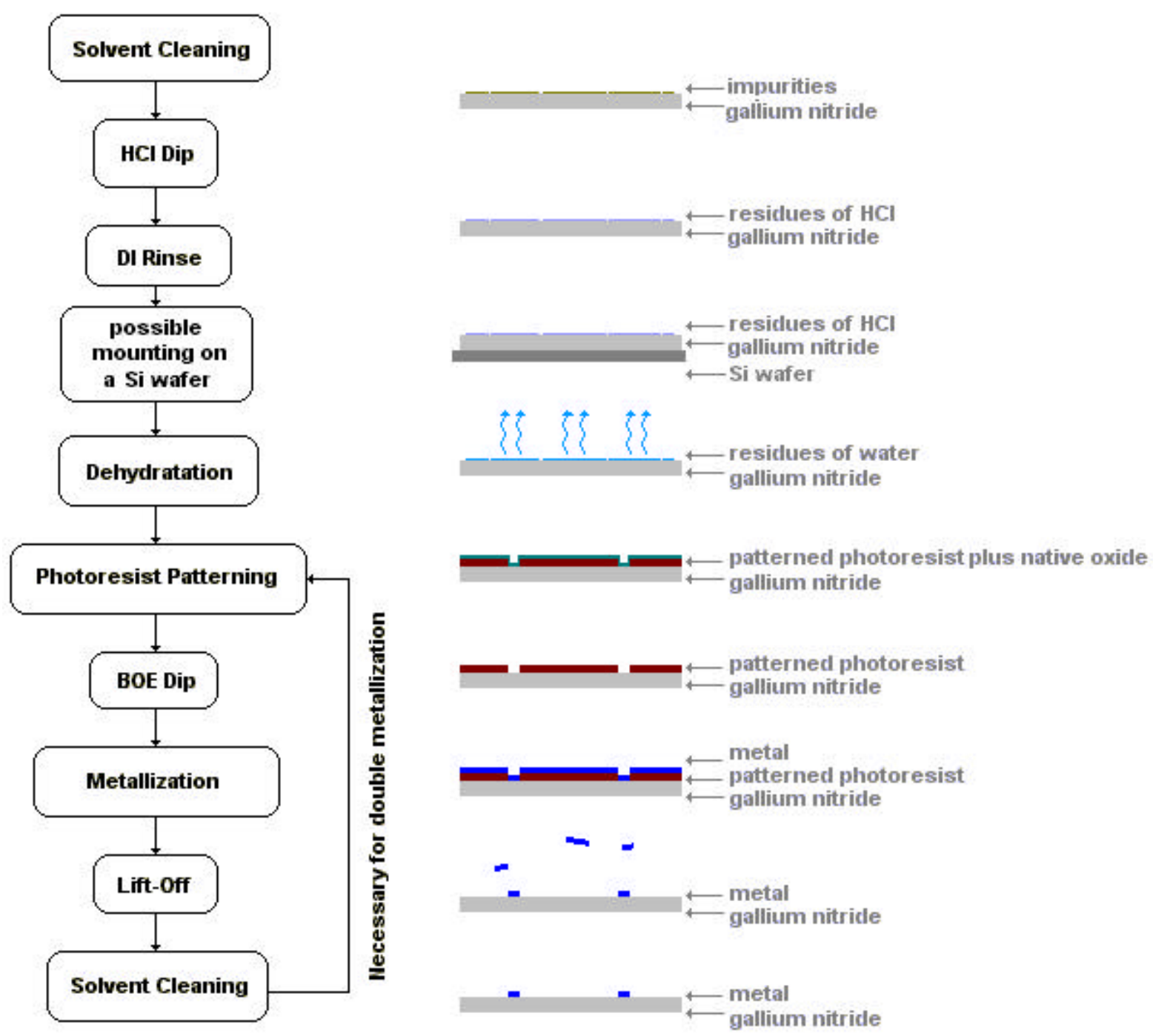

Figure 3.4: Flow diagram of preparation of gallium nitride samples.

The first cleaning step is made with trichloroethylene (TCE) [13][14][16]: A beaker is filled with TCE and heated at a temperature between $40^{\circ} \mathrm{C}$ and $50^{\circ} \mathrm{C}$. The temperature of the solution is measured by dipping the thermometer down to the middle of the beaker and waiting for a few seconds until equilibrium of temperature is reached between the thermometer and the solution. The thermometer never touches the bottom of the beaker to avoid any misreading. The sample stays in the solution for five minutes after the solution reaches the desired range of temperature. The sample is then blown dry and another beaker is filled with new TCE. The pyrex holder used to handle the sample is cleaned with de-ionized water (DI) for one minute, blown 
dry with a nitrogen gun, and then stays in a furnace at $130^{\circ} \mathrm{C}$ for one minute for a complete removal of the water.

The sample is loaded back in the holder and goes to the TCE solution. The beaker is then placed in an ultrasonic bath and is agitated for 5 minutes. As a final step the sample is removed from the holder with clean tweezers and blown dry with nitrogen.

Once the sample is cleaned with TCE it is cleaned with acetone [13][14][16]. This step is virtually the same as the TCE clean, except the solvent used is acetone in every stage. Following the cleaning with acetone, the sample is cleaned with methanol with the same procedure used for acetone above [13][14][16].

An $\mathrm{HCl}$ (hydrochloric acid) dipping is included in this cleaning process with the aim of striping the native oxide from the surface of $\mathrm{GaN}$ that is not usually removed with standard solvents [13][14][16]. The sample is dipped into a beaker full of $\mathrm{HCl}$ for five minutes at room temperature.

After the sample is cleaned with $\mathrm{HCl}$ it is necessary to do a cleaning with $\mathrm{DI}$ water to remove the acid from its surface and to avoid contamination of chlorine [13][14][15][16][17]. This rinse is performed in a dedicated beaker with flowing DI water for 5 minutes.

In order to remove the water from its surface, the piece is rotated in a spinner for one minute. This is preferable performed while blowing it dry with a nitrogen gun in order to perform it more quickly and more uniformly. Whenever the spinner cannot accommodate small pieces, they are mounted on a wafer of silicon. The wafer is placed on a hotplate previously heated at $100^{\circ} \mathrm{C}$. Next, wax (Quick Stick 135, composed of Ethylene Glycol and Phtalic Anhydride [21]) is melted on its top surface. The sample piece is then placed just above the region that contains the melted wax on the wafer. The sample is just dropped and is not pressed to avoid any damage of the structures or contamination of the surface. Finally, the wafer is removed from the hotplate and after one minute the wax solidifies and glues the sample to the wafer. 
Once the sample is cleaned and dry, it is coated with photoresist. This step is similar to that performed for silicon wafers. The only difference is the way the photoresist is deposited on the sample. First, the wafer is placed on the spinner and vacuum is generated to hold the wafer to the spinner. Then, the photoresist is loaded into a pipette and it is dropped on the center of the sample until it flows out the sample towards the wafer. This assures that the small samples are completely coated and the film thickness is uniform. The remaining steps of the photoresist patterning are exactly the same as that performed for silicon wafers.

After the patterns are developed, the sample is blown dry with a nitrogen gun. The sample is then dipped into a solution of hydrofluoric acid (1:100 HF:DI water) for 30 seconds to remove the native oxide from its surface [15][16][17]. Next the sample is rinsed with flowing DI water for 5 minutes. The sample is again blown dry with a nitrogen gun, and it is immediately loaded into a high vacuum chamber. The metallization is then performed exactly as for the silicon wafers.

The lift-off of the excess metal is the same performed for silicon wafers. However, if the sample was glued to a wafer with wax, then it is separated from the wafer, since the wax in use is soluble in acetone. The sample is detached from the silicon wafer by heating it on a hotplate and pulling the sample with clean tweezers.

As for silicon wafers, the GaN samples have to be degreased prior to a new metallization. The sample placed in a beaker full of acetone. This beaker stays heated at a temperature in the range of $40-50^{\circ} \mathrm{C}$ for five minutes. The sample is then blown dry with a nitrogen gun and then placed in a beaker full of methanol. The beaker once again stays heated at a temperature in the range of $40-50^{\circ} \mathrm{C}$ for 5 minutes and then blown dry again. A summary of the cleaning used for gallium nitride samples is shown on table 3.3. 
Table 3.3: Preparation process of GaN samples.

\begin{tabular}{|c|c|c|c|}
\hline Step & Chemical & Condition & Duration \\
\hline I & TCE & Warm & $5 \mathrm{~min}$ \\
\hline II & TCE & Bath & $5 \mathrm{~min}$ \\
\hline III & Acetone & Warm & $5 \mathrm{~min}$ \\
\hline IV & Acetone & Bath & $5 \mathrm{~min}$ \\
\hline V & Methanol & Warm & $5 \mathrm{~min}$ \\
\hline VI & Methanol & Bath & $5 \mathrm{~min}$ \\
\hline VII & HCl & Dip-RT & $5 \mathrm{~min}$ \\
\hline
\end{tabular}

\subsubsection{METALLIZATION OF G aN SAM PLES}

An appropriate choice of metals has to be made in order to create Ohmic and Schottky contacts to gallium nitride. All the GaN samples used in this work were unintentionally doped $n$ type. According to table 1.1, the bandgap of $\mathrm{GaN}$ is $3.4 \mathrm{eV}$. The electron affinity of $\mathrm{GaN}$ is $4.1 \mathrm{eV}$ [17]. Applying these values to equation 2.15a and solving it for the work function, one finds the root of the equation to be $4.1 \mathrm{eV}$. A metal having a work function close to this value would produce a good Ohmic contact to this semiconductor. The metal chosen was aluminum [13] [17] that has a work function of $4.5 \mathrm{eV}$. While not the best metallization alternative to achieve Ohmic contacts on n-type $\mathrm{GaN}, \mathrm{Al}$ was chosen due to its process simplicity that only involves a deposition. The resulting barrier height is $0.4 \mathrm{eV}$. This small barrier most of the time does not compromise the quality of the Ohmic contact. In cases when a Schottky behavior is observed, a rapid thermal annealing (RTA) can be performed at $900^{\circ} \mathrm{C}$ for 5 minutes [13][14]. This step was not needed or used in the current study.

As stated before in the case of silicon, when creating Schottky contacts one is interested in obtaining a reasonable barrier heigth. Solving again equation 2.15a for the work function, assuming a barrier height bigger than zero, one finds the work function to be bigger than $4.1 \mathrm{eV}$. The metal chosen was platinum [16][17]. The work function of platinum is $5.65 \mathrm{eV}$ [16], and is one of the highest in the periodic table. The resulting barrier height is calculated to be $1.55 \mathrm{eV}$ for this metal. 


\subsection{INTRUMENTATION}

Once the contacts are created on the surface of the semiconductor, they need to be measured to ensure their quality is good, and extract information such as FV (current vs. voltage) and $\mathrm{C}-\mathrm{V}$ (capacitance vs. voltage) from them. In order to measure $\mathrm{F}$ V and $\mathrm{C}-\mathrm{V}$ characteristics of semiconductors, two main pieces of equipment are used. These instruments are interfaced to a computer via GPIB using LabView. One instrument is a Multi - Frequency LCR Meter (HP4275A), and the other is a pA Meter / DC Voltage source (HP4140). The former is used to measure the capacitance of the device under test (DUT), and the latter is used as a bias source for C-V measurements, and as a system to take the I-V characteristic of the DUT.

\subsubsection{INSTRUMENTATION FOR C-V MEASUREMENTS}

The LCR meter has four probes, High Potential $\left(\mathrm{H}_{\mathrm{POT}}\right)$, Low Potential (LPOT), High Current $\left(\mathrm{H}_{\mathrm{CUR}}\right)$, and Low Current ( $\left.\mathrm{L}_{\mathrm{CUR}}\right)$. Every probe is shielded to ground. During its operation, the HP4275A applies an AC signal (OSC) to the sample through $\mathrm{H}_{\mathrm{CUR}}$ and ground. Added to this signal there is a bias voltage provided by the HP4140. Then probes Hот and LPOT measure the voltage drop along the DUT, whereas the current through DUT is measured by probe $\mathrm{L}_{\mathrm{CUR}}$ and ground. This mechanism is shown in figure 3.5.

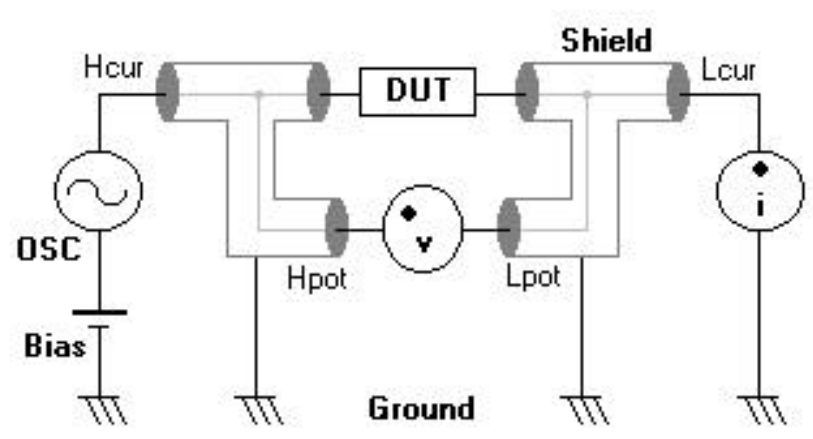

Figure 3.5: Basic operation of HP4275A. 
In the front panel of the HP4275A, one-meter long coaxial cables are connected to the four connectors. These four cables are then connected to a probe station where the DUT is placed. In the probe station housing and from about 10 centimeters from the DUT, $\mathrm{H}_{\mathrm{CUR}}$ and $\mathrm{H}_{\text {POT }}$ are shorted together, and a mini coax cable labeled "High" is connected between this short and the DUT. The same way, $\mathrm{I}_{\mathrm{CUR}}$ and $\mathrm{L}_{\text {POT }}$ are shorted together and a mini coax cable labeled "Low" is connected between this short and the DUT. Also, both shields and the substrate holder are connected to the ground. This configuration is also the setup recommended by the manufacturer of the equipment for high impedance measurements. Figure 3.6 shows the general connections among the HP4275A, the HP4140A, and the probing station. This figure as shows the specific grounding locations and where the cables are shorted inside the housing.

The test signal applied by HP4275 can be changed over a broad frequency range and voltage. The minimum and maximum $\mathrm{AC}$ voltages are $1 \mathrm{mV}$ and $1 \mathrm{~V}$ RMS respectively, and the minimum and maximum frequencies are $10 \mathrm{KHz}$ and $10 \mathrm{MHz}$ respectively. The typical value for the test voltage (OSC) is $100 \mathrm{mV}$, and the test frequency is changed according to the nature of measurement (see chapter 2). 


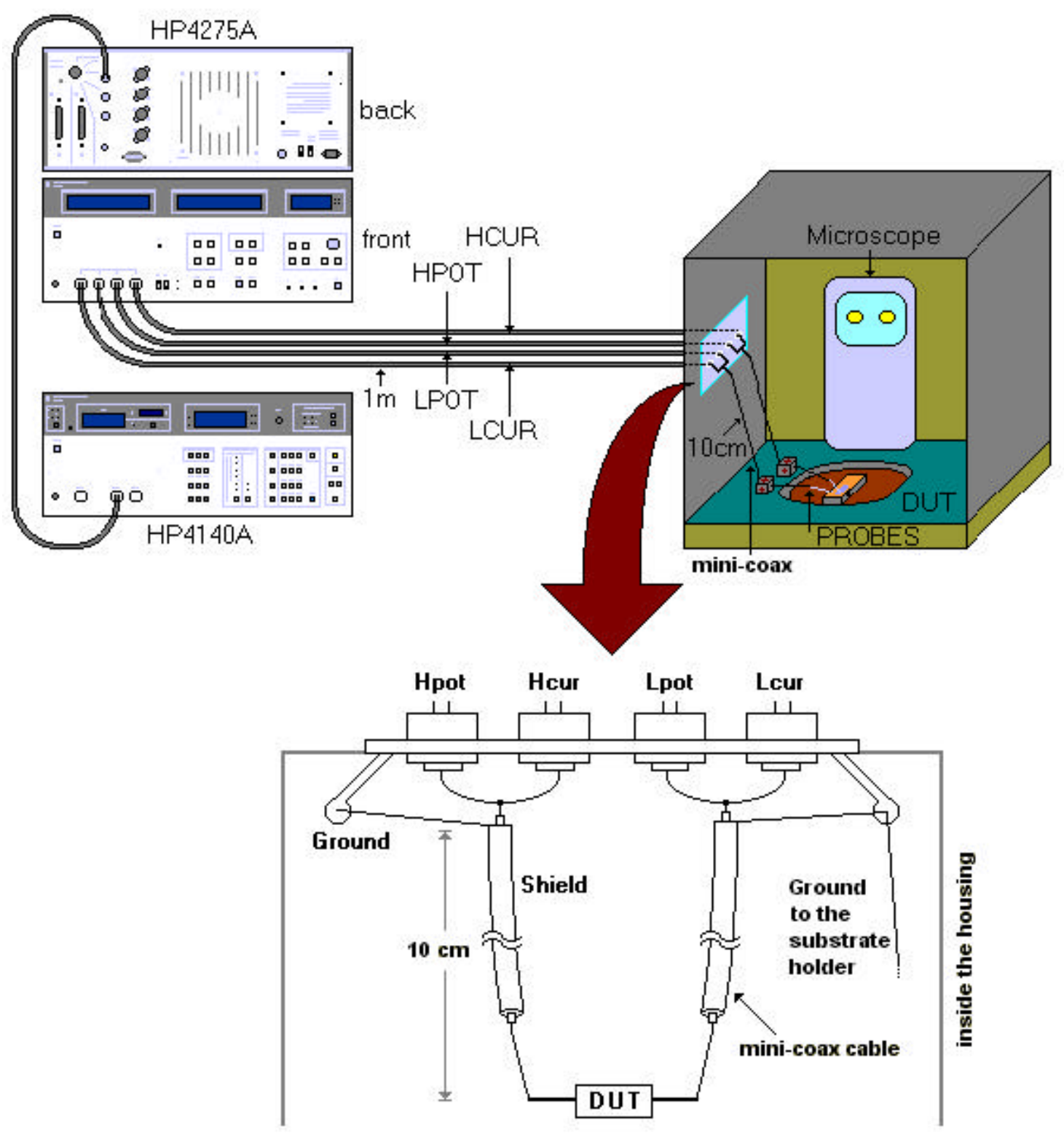

Figure 3.6: General connection among the two HPs and the probing station for C-V measurements. 
When cables are connected to the instruments, parasitic capacitances, impedances and inductances are introduced in the measurement and their effect must be canceled. The HP4275a provides a procedure to cancel these parasitic components. First the bias voltage has to be turned off (back of the equipment), and the test voltage level has to be turned all the way clockwise to the highest test voltage. Then the ends of the two cables (High and Low) have to be in the approximate position in which they will be during the measurement but not touching each other. When the cables are in this open-circuit condition, "zero open" is pressed (front of equipment). The HP4275 then performs tests over all working frequencies and the equipment is ready for the second calibration. Now with the two ends shorted in a position close to their measurement position, "zero short" is pressed (front of equipment). Again the HP4275 performs tests over all working frequencies. With the completion of this procedure, the equipment nulls the parasitic contribution of the cables, and is ready to take measurements. This must be done each time the cables or probes are moved or samples changed.

For measuring metal contact characteristics the first thing to be verified is the kind of contact under measurement. For Ohmic contacts there is no specific contact configuration, and the probes can be placed in any order on the contacts. For Schottky contacts on the other hand, one needs to know which metal is the anode (positive) contact and which is the cathode (negative) contact. A simple rule is to verify what type the bulk is and which metal behaves as the Ohmic contact.

The Ohmic contact assumes the type of the bulk because ideally there is no voltage drop in an Ohmic contact. The Schottky contact, on the other hand, assumes the inverse polarity of the bulk because of the redistribution of charges as discussed in the previous chapter. For instance, if a particular bulk is n-type, aluminum forms an Ohmic contact, and platinum forms an Schottky contact to it, then aluminum behaves as the $\mathrm{r}$ side of the junction, and platinum behaves as the $\mathrm{p}$ side. Figure 3.7 illustrates one example of configuration of the probes on the device under test.

Once the polarities of the contacts have been determined, the probes have to be placed accordingly. The "high" probes have to touch the positive side of the junction, and the "low" probes have to touch the negative side of junction. 
The dissipation factor is an important piece of information that provides the reactive part of the circuit divided by the impedance part of the circuit. As described in Chapter 2, the dissipation factor indicates how well the parallel circuit model applies to the device under test. Prior of taking $\mathrm{C}-\mathrm{V}$ curves and extracting information from them, one should take a measurement of the dissipation factor $v s$. frequency. This curve says how the loss tangent changes with frequency and therefore at what frequencies the equipment produces a reading that best represents the physical properties of DUT. It is recommended by the manufacturer of the HP4275 that the dissipation factor should be less than 0.1 for an accuracy of $\pm 0.1 \%$. The accuracy of the $\mathrm{C}-\mathrm{V}$ measurement is proportional to $1+\mathrm{D}^{2}$ for values of the dissipation factor bigger than 0.1 . Therefore, the minimum of the curve of dissipation factor $v s$. frequency can be found, and measurements of capacitance at this frequency should produce results with the highest accuracy. This is especially important when measuring the built-in voltage, since the value of the capacitance at zero bias is necessary for this measurement.

When taking $\mathrm{C}-\mathrm{V}$ measurements, one is interested in the region between the breakdown voltage and the threshold voltage of the device as discussed in session 2.3. Therefore, the probes should be configured as discussed, and the applied voltage should stay between these two values.

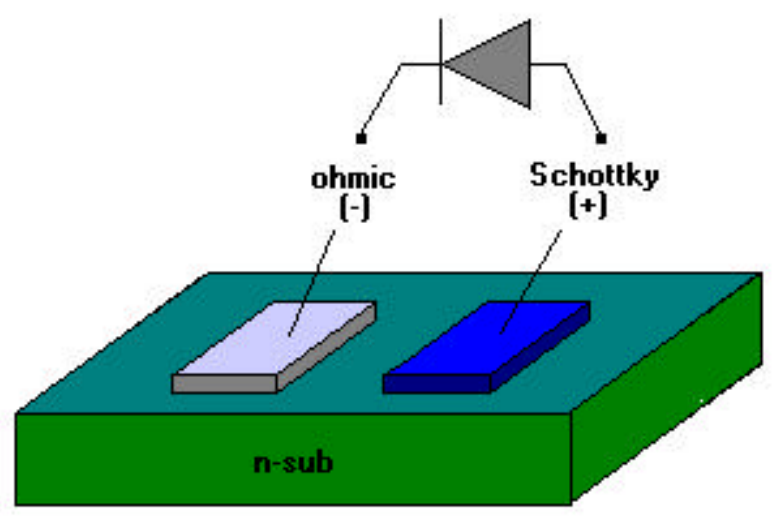

Figure 3.7: Example of probing configuration. 
A program written in LabView executes the control of the measurement. This program is listed in appendix 6.2. In this program, one enters the initial value of the applied voltage, the final value, and the voltage step. Also, the user has the flexibility of setting the frequency of the test voltage (OSC) and the delay between two capacitance readings. The default delay used in the program is 500 milliseconds. Power is then pressed on, the program is run, and the on/off key on the LabView program is switched on. The program returns the value of capacitance for every applied bias as well as a plot of capacitance $v s$. voltage and a plot of $1 / \mathrm{C}^{2} v s$. voltage.

Once the program finishes taking a measurement, the user has the option to save the measurement in a dialog box. This file contains one column for the applied voltage, one column for the capacitance corresponding to that bias voltage, and a column for the dissipation factor (D).

This file is imported into a graphics manipulator program such as Sigma Plot. A capacitance vs. voltage is plotted in order to determine whether the measurement produced a valid result. Then, $1 / \mathrm{C}^{2}$ is plotted against the applied voltage. This plot should produce a straight line as shown by equation 2.19a. From this plot, two pieces of information are extracted: the slope of the curve, and the intercept point of the curve to the capacitance axis.

These two data are then used in a program written in $\mathrm{C}$ language (Appendix C) to determine the carrier concentration, built-in voltage, and depletion width. The program first asks for the radius of the dot that was measured. With this information it calculates the area of the dot. The program then asks for the substrate. According to this information the program uses the appropriate dielectric constant. The next two pieces of information needed are the slope and the intercept of the $1 / \mathrm{C}^{2}$ plot.

The program applies equation $2.19 \mathrm{~b}$ to calculate the carrier concentration, $2.20 \mathrm{~b}$ to calculate the built-in voltage, and equation $2.14 \mathrm{~b}$ to calculate the depletion layer width. 


\subsubsection{INSTRUMENTATION FOR I-V MEASUREMENTS}

The HP4140A has three connectors. There are two power supplies $\mathrm{V}_{\mathrm{A}}$ and $\mathrm{V}_{\mathrm{B}}$, and a current meter I. For $\mathrm{C}-\mathrm{V}$ measurements it is only necessary to connect $\mathrm{V}_{\mathrm{A}}$ to the bias input of HP4275, whereas for I-V measurements the configuration is quite different.

When taking I-V measurements, one is interested in applying a voltage to the DUT and measures the resulting current. Therefore, a standard configuration for the HP4140A is to

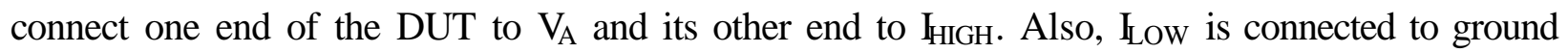
to close the circuit. This configuration can be seen in figure 3.8. The connection between the HP4140A and the probing station is shown in figure 3.9.

For IV measurements, only the HP4140A is necessary. $\mathrm{V}_{\mathrm{A}}$ connector is a coax, whereas the current cable is a triax as shown in figure 3.9. The shields are grounded and shorted to Low at a microprobing station. Attention is taken to warm up times. As recommended by the manufacturer, the equipment should warm up for at least one hour prior to any measurement.

The configuration of the probes is as specified for $\mathrm{C}-\mathrm{V}$ measurements. The potential probe $\mathrm{V}_{\mathrm{A}}$ is connected to the positive side of the junction, whereas the $\mathrm{I}_{\mathrm{HIGH}}$ terminal is connected to the negative side of the junction. The measurement is taken at any range of voltages as desired. Attention should be taken to the corresponding current in order to maintain it within an acceptable range. A typical current ranges from $-0.01 \mathrm{~A}$ to $0.01 \mathrm{~A}$.

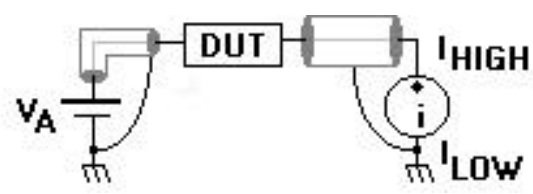

Figure 3.8: Circuit model of the measurement equipment. 

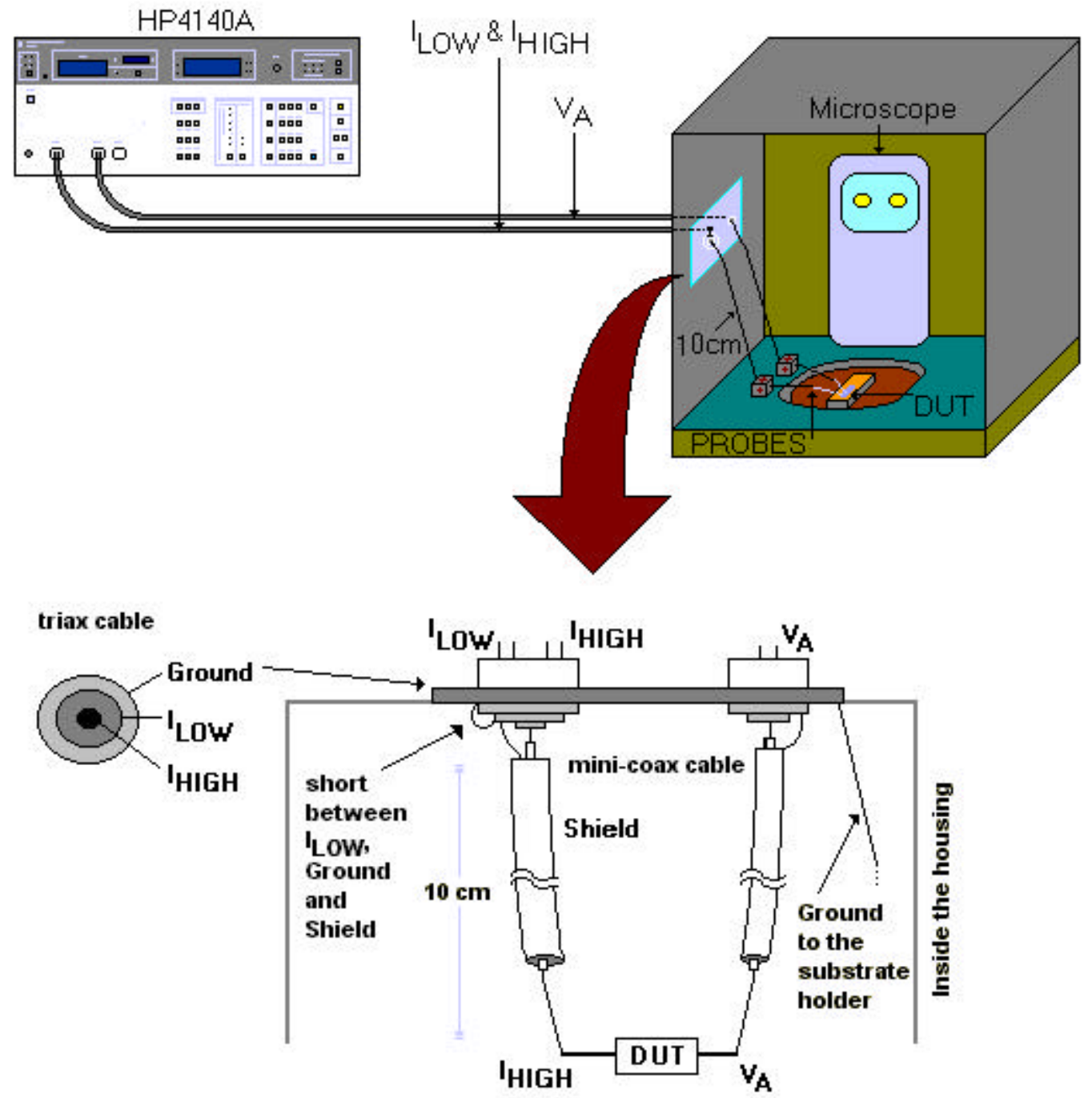

Figure 3.9: General connection between HP4140A and the probing station.

A program written in LabView executes the control of the measurement. This program is listed in appendix 6.1. In this program, one enters the initial value of the applied voltage, the final value, and the voltage step. Power is switched on, the program is set to run and the on/off key is switched. The HP4140A measures a corresponding current at each voltage point, and a plot of current vs. voltage is shown on the screen of the computer. Also, a logarithmic plot of the current $v s$. voltage is displayed so that the user can visualize more detailed differences in the low current portions of the plot. At the end of its execution, the program opens a dialog window that allows the user to save the I-V characteristic of the device under test. 
After the $\mathrm{FV}$ file is saved, a graphics manipulator program is used to analyze the data. As shown by equation 2.4, the series resistance can be extracted directly from the FV plot. Also, it is possible to estimate the built-in and the breakdown voltages as stated in chapter 2 . In the case when Ohmic circular planar structures are being measured, equation 2.35 is used to calculate the specific contact resistance and the sheet resistance of the material. The resistance of a set of dots are measured from their FV curves and then are fit with their separations between the their dots and rings into equation 2.35. 


\section{Chapter 4 - Results}

\section{$4.1 \quad$ INTRODUCTION}

A theoretical framework was detailed in Chapter 2 for a set of measurements such as sheet resistance, specific contact resistance, capacitance, built-in voltage, and carrier concentration. The experimental procedures and instrumentation setups necessary to perform these measurements were discussed thoroughly in the previous chapter. Using the framework developed in Chapter 2 in combination with the instrumentation techniques discussed in Chapter 3, some samples were measured to compare the theoretical predictions with real measured values. Prior the processing of highly resistive samples, a wafer of gallium arsenide with high carrier concentration and low bulk resistivity was processed to check a situation close to the ideal case where the series resistance is small. The results found for gallium arsenide are presented on Appendix D.

\subsection{SILICON TEST SAM PLE}

In order emulate the behavior of highly resistive materials such as unintentionally doped n-type gallium nitride, a sample of silicon with low carrier concentration was processed. This sample was a 3-inch diameter, (100) orientation silicon wafer, boron doped, nominal carrier concentration between $6.3 \times 10^{14} \mathrm{~cm}^{-3}$ and $10^{15} \mathrm{~cm}^{-3}$, and nominal resistivity between $14 \Omega-\mathrm{cm}$ and $22 \Omega-\mathrm{cm}$. The sample was cleaned and prepared as described in section 3.2. The Ohmic contact was alloyed aluminum, and the Schottky contact was chromium. The second mask design (fig. 3.2) was used to pattern the contacts.

\subsubsection{RESISTANCE MEASUREMENTS}

The first measurement performed on this sample was an I-V analysis of five Ohmic contacts to extract the sheet resistance, and the specific contact resistance of the material. The

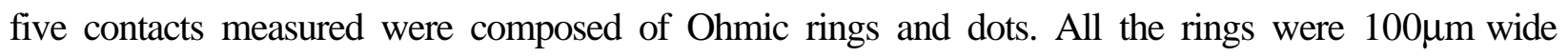
separated by $40 \mu \mathrm{m}$ from the dots. The radii of the dots were $250 \mu \mathrm{m}, 200 \mu \mathrm{m}, 150 \mu \mathrm{m}, 100 \mu \mathrm{m}$, 
and $50 \mu \mathrm{m}$. FV curves were taken for each of these structures, and the total resistances were then extracted directly from these curves by a fitting routine over the linear range of the curve.

Figure 4.1 shows the measured FV curves, and the total measured resistances are shown in table 4.1. These curves saturate above 0.01A. This happens because this is the maximum current the HP4140A can handle.

Table 4.1: Total resistance versus radius of five Ohmic contacts on silicon.

\begin{tabular}{|c|c|}
\hline Radius $[\mu \mathrm{m}]$ & Total Resistance $[\Omega]$ \\
\hline 250 & 128.22 \\
\hline 200 & 172.12 \\
\hline 150 & 217.98 \\
\hline 100 & 360.50 \\
\hline 50 & 795.73 \\
\hline
\end{tabular}

The values of resistance from table 4.1 were then fit with equation 2.38 to find the sheet resistance and the specific contact resistance. Figure 4.2 shows a plot of this fit. The values found for the sheet resistance and specific contact resistance were $2.8 \mathrm{~K} \Omega$ and $2.25 \times 10^{-2} \Omega-\mathrm{cm}^{-2}$ respectively. The constant $\mathrm{L}_{\mathrm{T}}$ in this case was $28.3 \mu \mathrm{m}$. Since it is approximately 9 times smaller than the dot radius $(250 \mu \mathrm{m})$, the use of equation 2.38 is justified. 
Si NewMask2

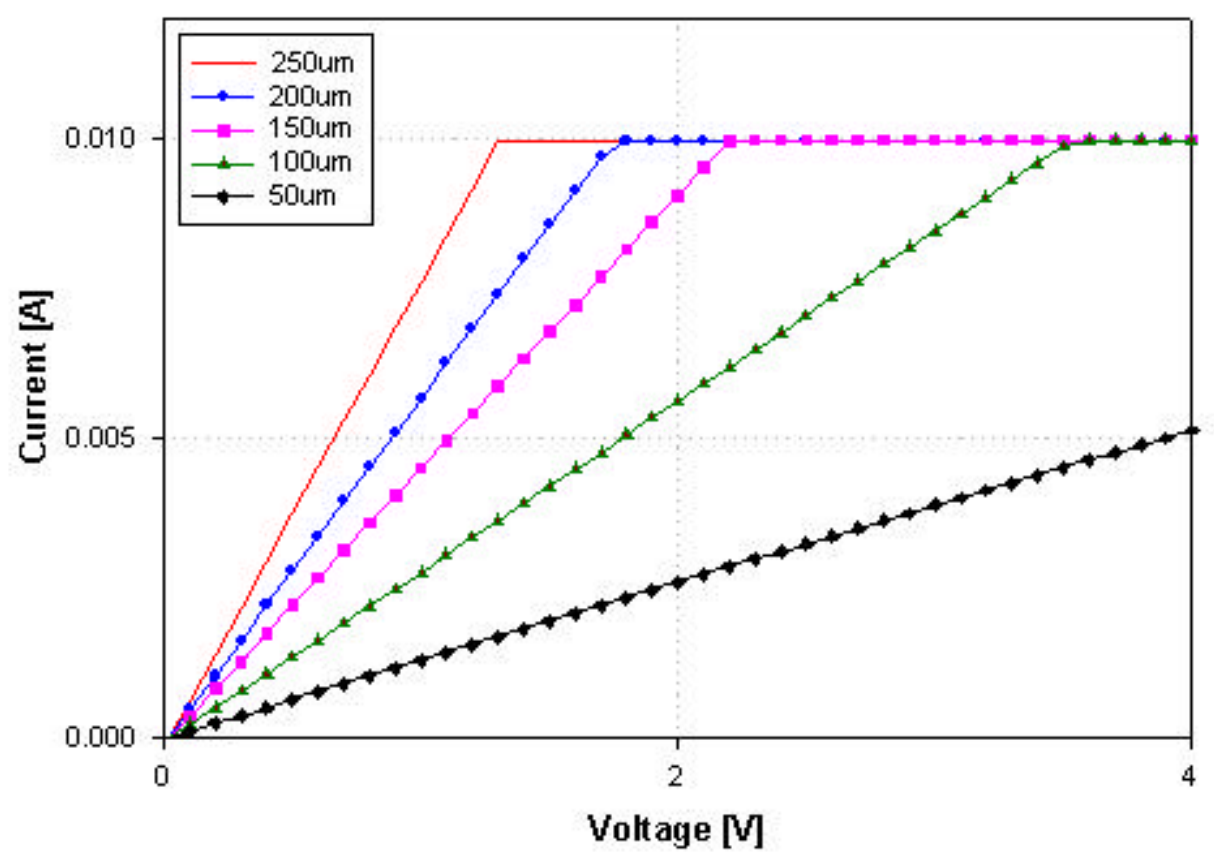

Figure 4.1: I-V curves of five Ohmic contacts on Si.

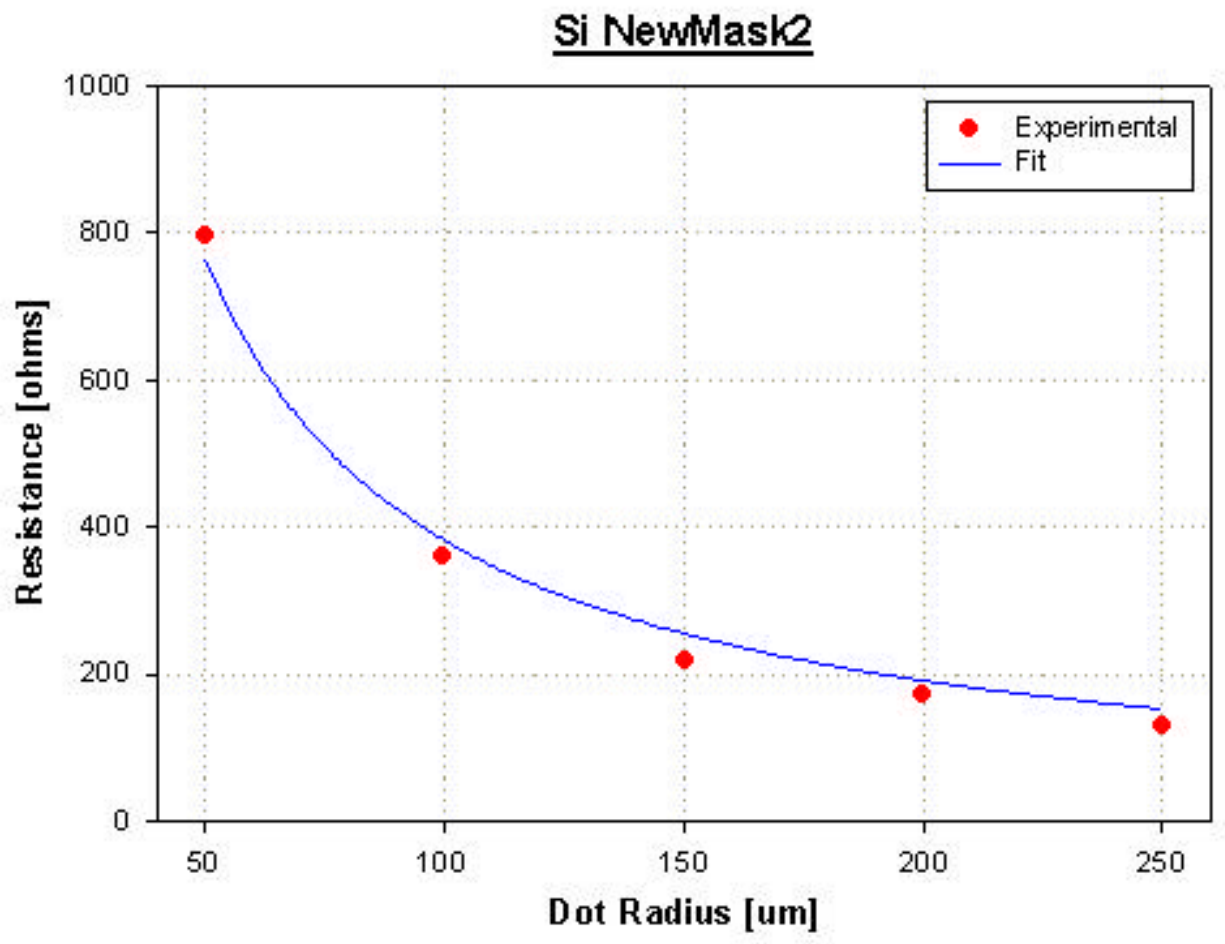

Figure 4.2: Fitting of resistance versus dot radius on $\mathrm{Si}$. 


\subsubsection{SCHOTTKY MEASUREMENTS}

Following the I-V measurements on Ohmic contacts, a second set of I-V measurements was taken on Schottky contacts to verify their proper behavior. Four contacts were measured. All of them had a $100 \mu \mathrm{m}$ wide Ohmic ring, a Schottky dot with $250 \mu \mathrm{m}$ or radius, and separations of $20 \mu \mathrm{m}, 50 \mu \mathrm{m}, 60 \mu \mathrm{m}$, and $100 \mu \mathrm{m}$ between the dot and the ring. The plots of the log $\mathrm{FV}$ and linear

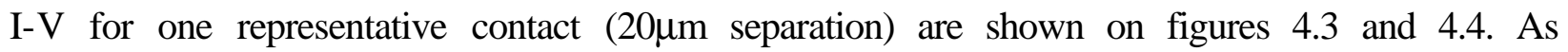
expected, the saturation current was very small. In this case it was smaller than $10^{-7} \mathrm{~A}$.

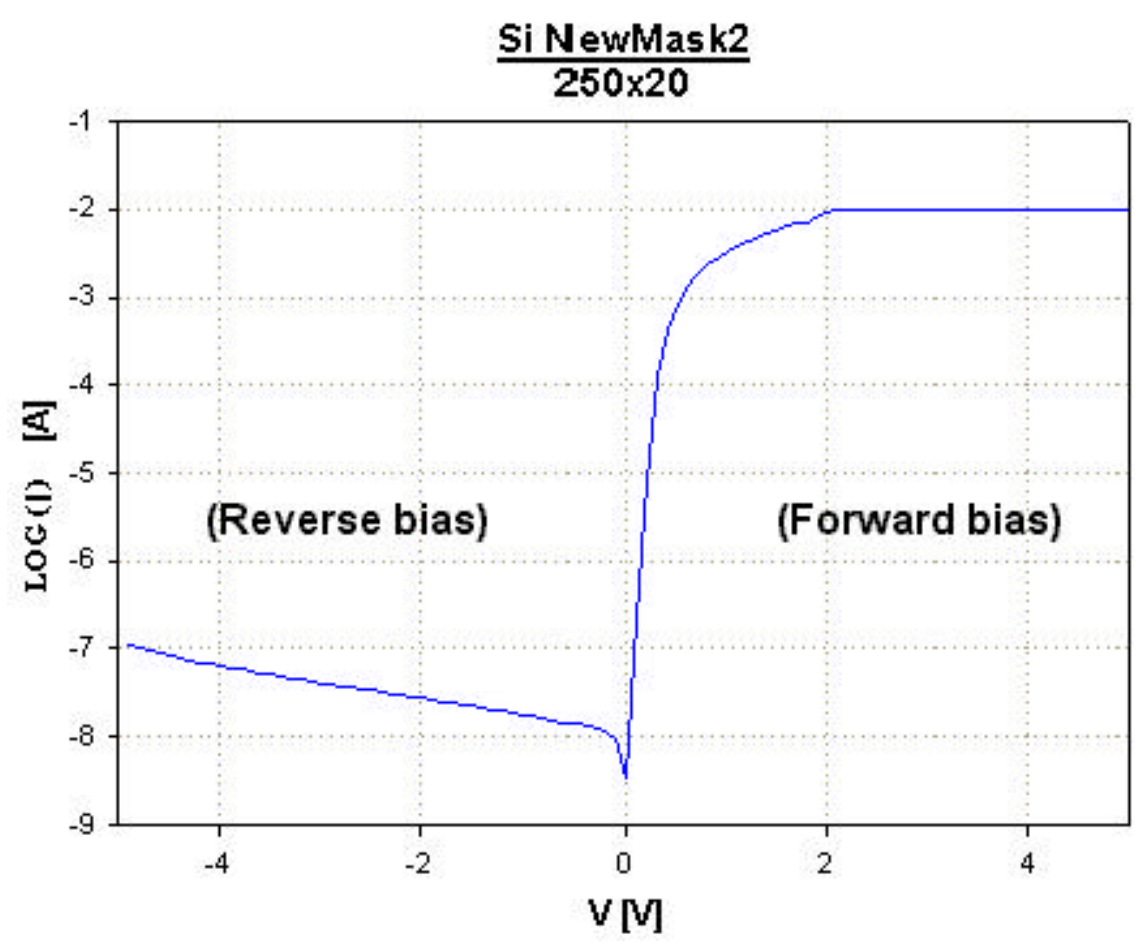

Figure 4.3: $\mathrm{Log} \mathrm{I}-\mathrm{V}$ plot of a $250 \mu \mathrm{m}$ dot with $20 \mu \mathrm{m}$ of separation on Si. 


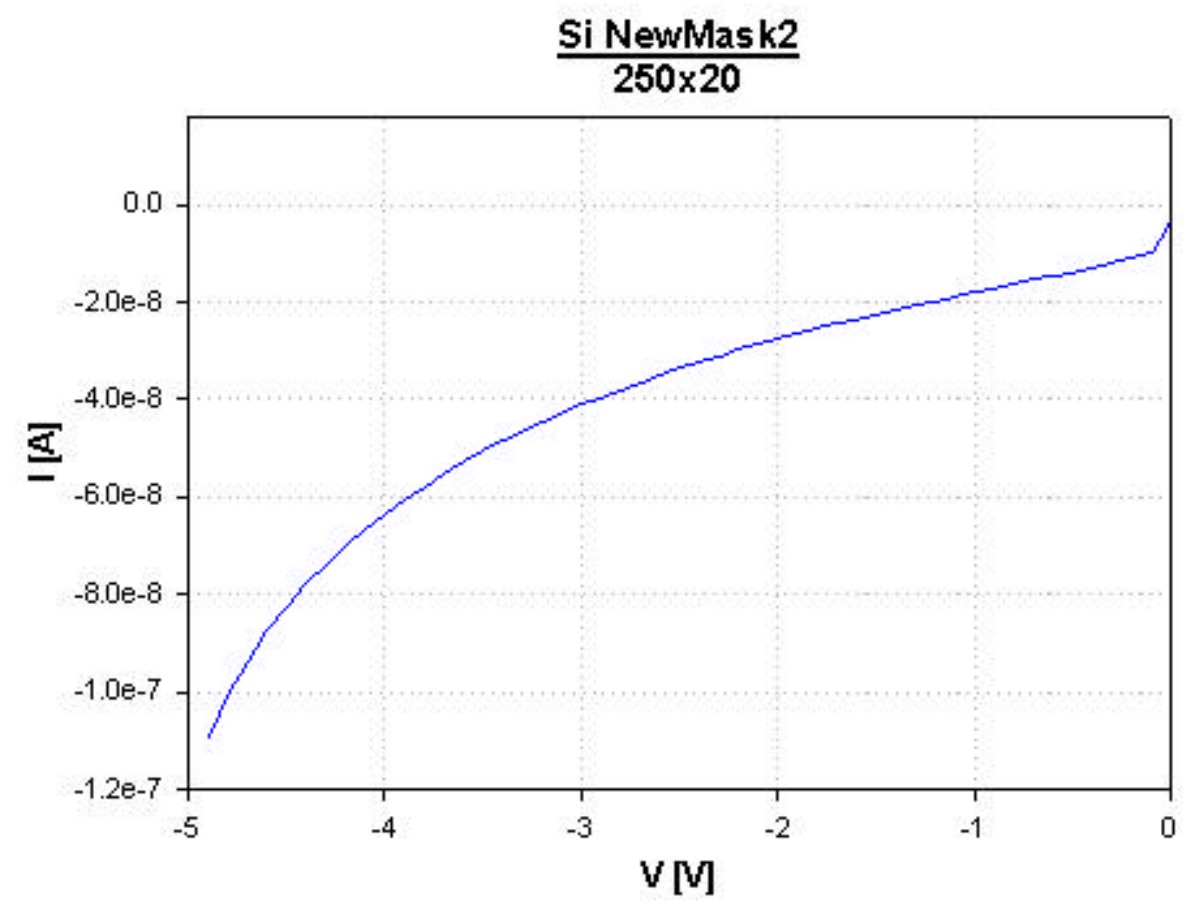

Figure 4.4: Saturation current of a $250 \mu \mathrm{m}$ dot with $20 \mu \mathrm{m}$ of separation on $\mathrm{Si}$.

\subsubsection{LOSS TANGENT}

One of the most important pieces of information that can be extracted from a Shottky contact is the junction capacitance. With the junction capacitance, as demonstrated in Chapter 2, it is possible to determine some important parameters such as the built-in voltage of the junction, and the carrier concentration of the material. However, before any measurement of capacitance takes place, one should first analyze how the loss tangent of the DUT changes with frequency. Also, as stated in table 2.1 , the frequency should be reasonably small, and the capacitance has to be measured in the flat region of the capacitance versus frequency curve.

The first point to be analyzed, the loss tangent, is the ratio of the capacitive reactance to the parallel resistance of the junction. The loss tangent says how leaky the capacitance is, and how accurate the measurement can be. Remembering equation 2.27 from Chapter 2, the expected shape of the loss tangent versus frequency should have a distinct minimum of frequency where the measurements produce a more accurate capacitance reading. The dissipation factor was then measured against frequency at no applied bias. A representative plot, obtained from the same 
structure $(250 \mu \mathrm{m}$ dot with $20 \mu \mathrm{m}$ of separation between the dot and the ring), is shown in picture 4.5 .

It can be observed from the graph that the loss tangent changes with frequency as predicted in Chapter 2. At low frequencies, the $1 / \omega$ term in equation 2.27 is dominant and the corresponding shape is a hyperbola. As the frequency starts to increase, the capacitance decreases, the conductance increases, the quadratic term in equation 2.27 therefore increases, and as a consequence the curve of dissipation factor reaches a minimum and starts to bend back to bigger values. The frequency at which the loss tangent reaches its minimum is the frequency that has to be used in further measurements of the capacitance in order to obtain reliable and accurate capacitance readings. Another option is to measure the dissipation factor at each applied bias and then use equation 2.21e to find the value of the real depletion capacitance at a specific frequency. This last alternative, however, does not take into account the accuracy of the measurement.

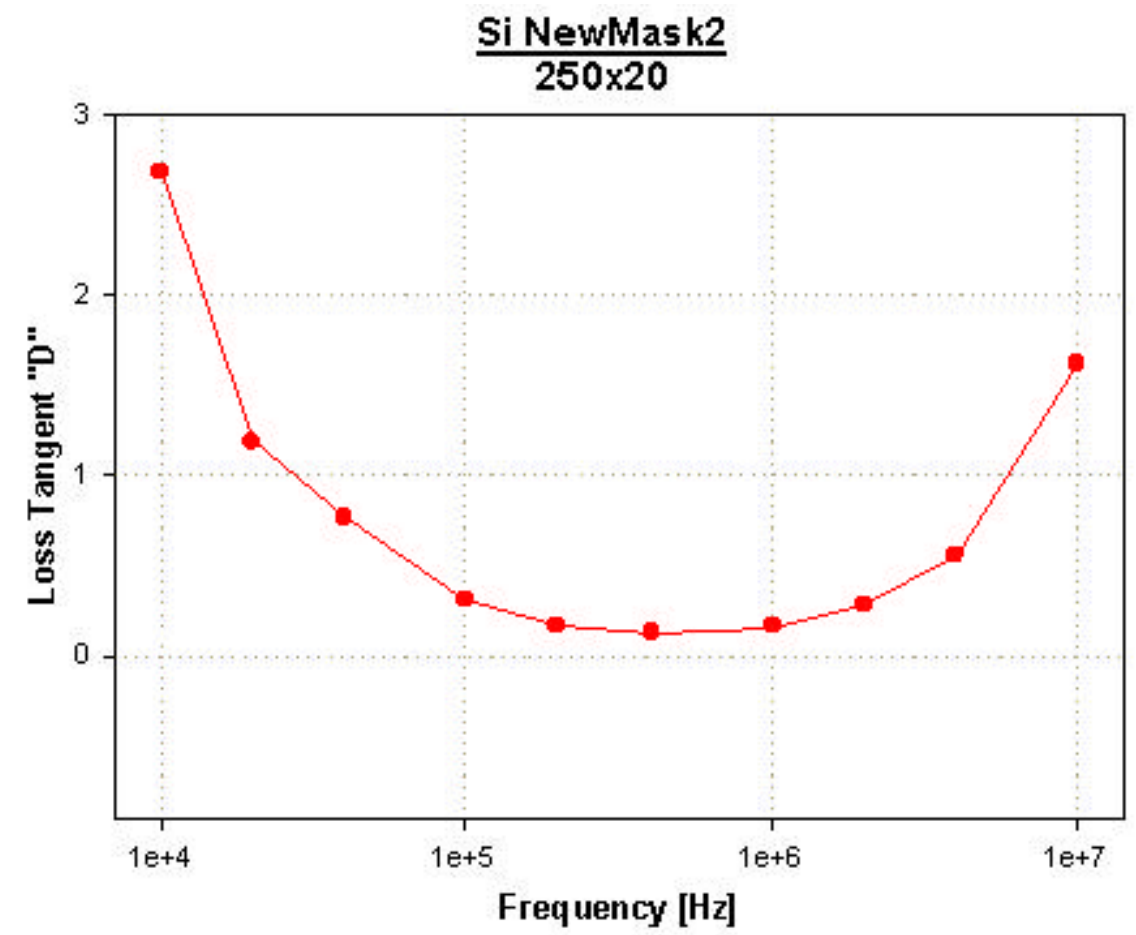

Figure 4.5: Loss tangent versus frequency of a $250 \mu \mathrm{m}$ dot with $20 \mu \mathrm{m}$ of separation on Si. 


\subsubsection{DEPLETION CAPACITANCE AT ZERO BIAS}

Following the criteria necessary to perform a reliable measurement of capacitance, the depletion capacitance has to be analyzed in he case where there is no bias applied. This situation is particularly important for the case where one is interested in calculating the built-in voltage of

a junction. In order to perform such measurement, the depletion capacitance at zero bias is an essential piece of information.

As discussed in section 2.3.1 and shown on figure 2.22, the measured junction capacitance is approximately flat at low frequencies, but has a decay shape as the frequency is increased. Also, equation 2.21c and figure 2.29 show that the total junction conductance increases as the frequency increases, and this in turn contributes to the increase of the loss tangent. Therefore the measurement of the capacitance has to be taken in a flat region of these two plots so that the frequency-dependence of the capacitance and conductance do not dominate the measurement. The plots of the measured capacitance and conductance as functions of the frequency of a representative contact $(250 \mu \mathrm{m}$ and $20 \mu \mathrm{m}$ of separation) are shown on figures 4.6 and 4.7. It can be seen for this sample that points between $10 \mathrm{KHz}$ and $1 \mathrm{MHz}$ should produce the most reliable measurement. Also, the product $\mathrm{rG}$ can be calculated for the worst case when $\mathrm{r}=$ $128.22 \Omega$ as given on table 4.1 , and $\mathrm{G}=5 \times 10^{-4} \Omega^{-1}$. The product $\mathrm{rG}$ calculated in this case was $0.064(<<1)$ and therefore the measurements should not be affected by the series resistance. Applying these values of the series resistance and parallel conductance to equation 2.21 and using a frequency of $1 \mathrm{MHz}$, one finds that the measured capacitance is approximately only $12 \%$ higher than expected.

\subsubsection{BUILT-IN VOLTAGE}

In order to find the built-in voltage associated with a Shottky contact, one first needs to find the depletion capacitance at zero applied bias. Analyzing figures 4.5 and 4.6 one finds the point at which the loss tangent is the minimum and the capacitance is in a flat portion of the curve. Four contacts were measured. All contacts had a dot with $250 \mu \mathrm{m}$ of radius, but different separations between their dots and rings. The separations were $20 \mu \mathrm{m}, 50 \mu \mathrm{m}, 60 \mu \mathrm{m}$, and $100 \mu \mathrm{m}$. 


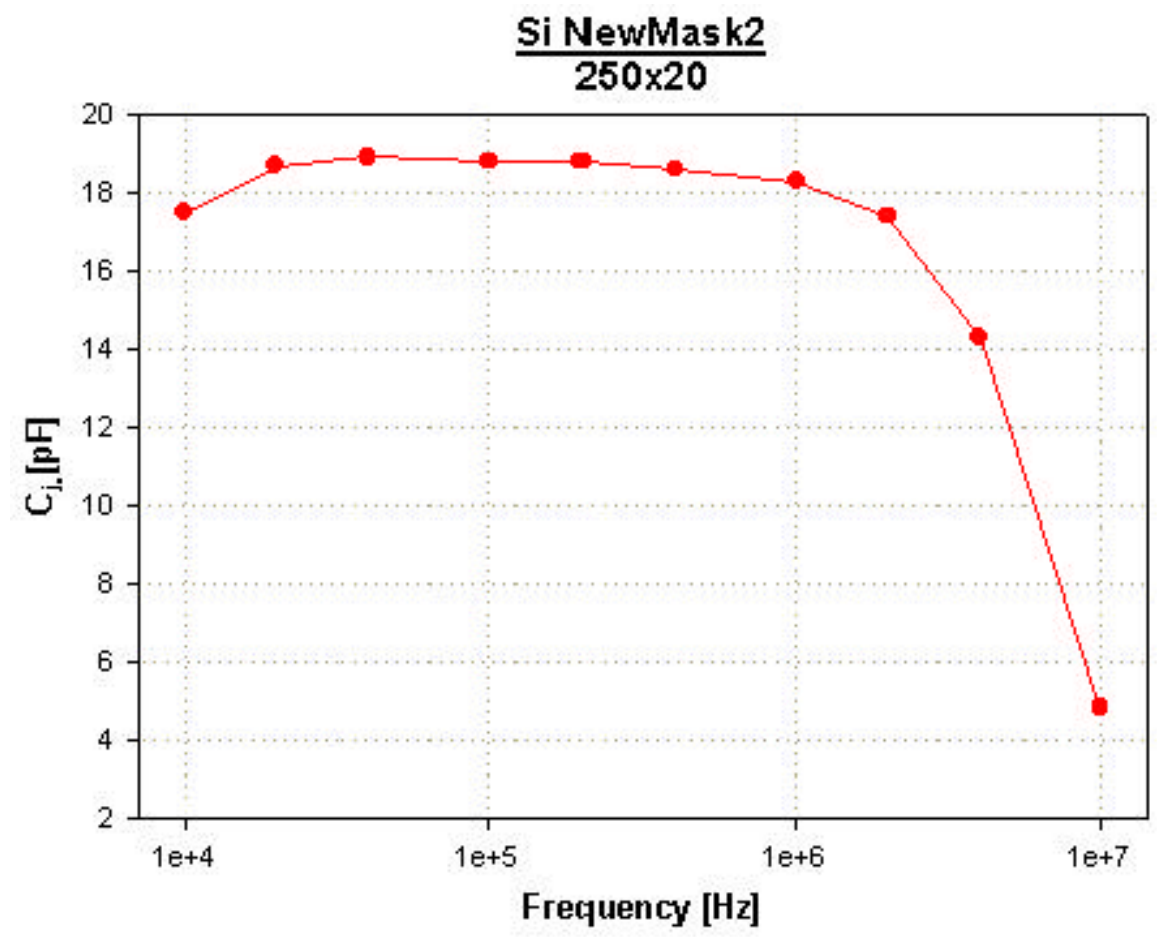

Figure 4.6: Junction capacitance measured on a $250 \mu \mathrm{m}$ dot with $20 \mu \mathrm{m}$ of separation on $\mathrm{Si}$ at zero bias.

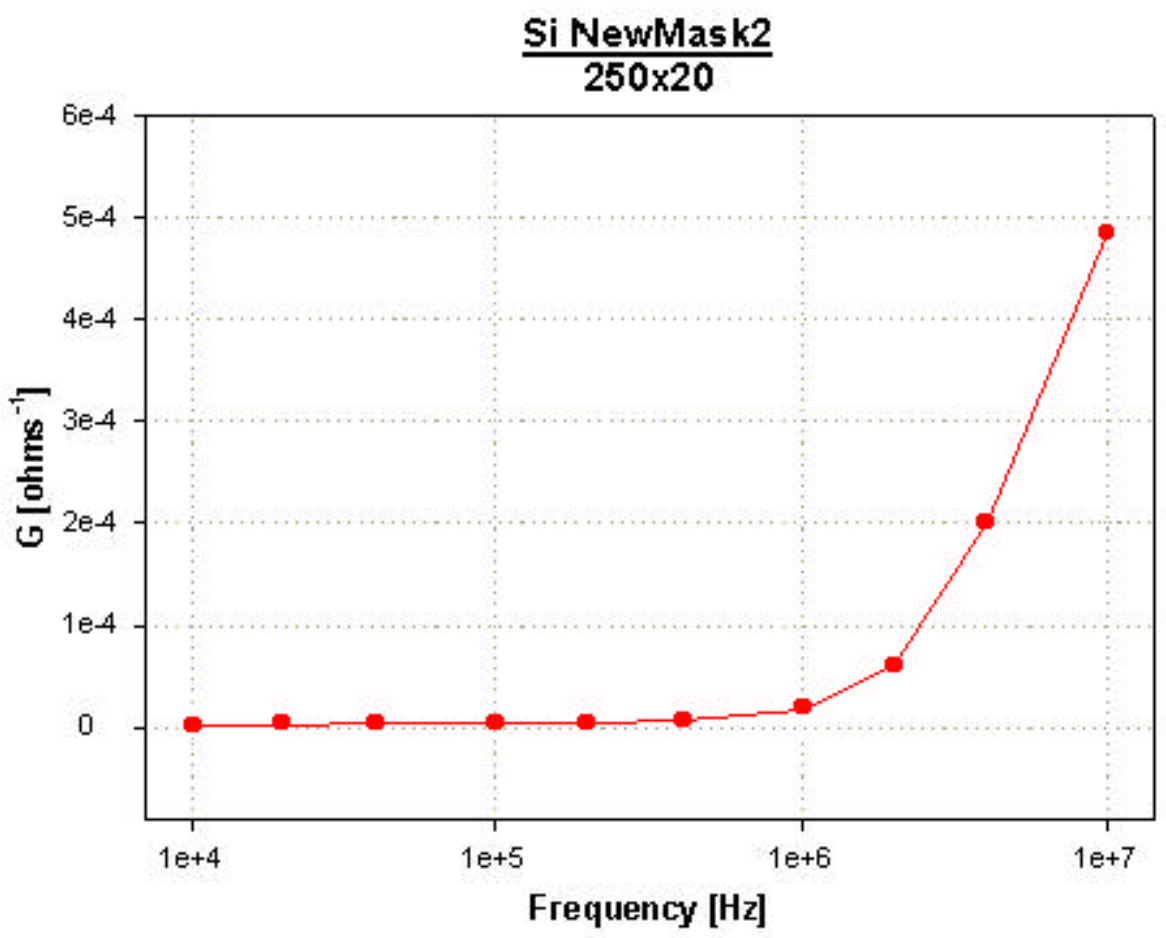

Figure 4.7: Junction conductance measured on a $250 \mu \mathrm{m}$ dot with $20 \mu \mathrm{m}$ of separation at zero bias. 
The frequencies of the lowest losses determined for the built-in voltage measurement for each of these contacts are shown on table 4.2.

Table 4.2: Points of lowest losses for the measured contacts on Si.

\begin{tabular}{|c|c|c|c|}
\hline Separation $[\mu \mathrm{m}]$ & Frequency $[\mathbf{H z}]$ & Loss tangent 'D" & Measured $\mathbf{C}_{\mathbf{j o}}[\mathbf{p F}]$ \\
\hline 20 & $400 \mathrm{~K}$ & 0.13 & 18.6 \\
\hline 50 & $1 \mathrm{M}$ & 0.17 & 18.5 \\
\hline 60 & $400 \mathrm{~K}$ & 0.27 & 18.3 \\
\hline 100 & $1 \mathrm{M}$ & 0.19 & 18.5 \\
\hline
\end{tabular}

The calculated mean and standard deviation of the loss tangent were 0.19 and 0.05 respectively. The calculated mean and standard deviation for the measured capacitance at no bias were $18.47 \mathrm{pF}$ and $0.11 \mathrm{pF}$ respectively. Based on the results from table $4.2, \mathrm{C}-\mathrm{V}$ curves were taken at those specific frequencies. The $\mathrm{C}-\mathrm{V}$ curve of a representative contact $(250 \mu \mathrm{m}$ dot with $20 \mu \mathrm{m}$ of separation) is shown on figure 4.8. Having the $\mathrm{C}-\mathrm{V}$ curve and remembering equation $2.20 \mathrm{~b}$, one also needs to plot a curve of $\mathrm{C}^{2} v s$. V to find the slope and intercept with the voltage axis. The plot of $\mathrm{C}^{2} v s$. $\mathrm{V}$ obtained from the same contact is shown on figure 4.9. At this point it is necessary to consider a possible case when the $\mathrm{C}^{2} v s$. V curve is bent. Remembering equation $2.32 \mathrm{~b}$, if the saturation current is high enough, the diffusion capacitance contributes significantly. Also, it has an exponential dependence with the bias voltage that in turn bends the C-V and the $\mathrm{C}^{-2}$ vs. $\mathrm{V}$ curve. That was not the case on the measured contacts. The values obtained for the slope and intercept for each of the $\mathrm{C}^{2}$ vs. $\mathrm{V}$ curves are shown on table 4.3. Using the values of table 4.3, a program (appendix C) applies equation $2.20 \mathrm{~b}$ to calculate the built-in voltage. These results are found on table 4.4 .

Table 4.3: Slope and intercept of $\mathrm{C}^{-2} v s$. V curves of four contacts on Si.

\begin{tabular}{|c|c|c|}
\hline Separation $[\mu \mathrm{m}]$ & Slope $x 10^{21}\left[F^{-2} V^{-1}\right]$ & Intercept $x 10^{21}\left[\mathrm{~F}^{-2}\right]$ \\
\hline 20 & -3.66 & 3.44 \\
\hline 50 & -3.64 & 3.46 \\
\hline 60 & -3.63 & 3.46 \\
\hline 100 & -3.65 & 3.46 \\
\hline
\end{tabular}


Table 4.4: Calculated built-in voltages for the measured contacts on Si.

\begin{tabular}{|c|c|}
\hline Separation $[\mu \mathbf{m}]$ & Built-in Voltage [V] \\
\hline 20 & 0.97 \\
\hline 50 & 0.98 \\
\hline 60 & 0.98 \\
\hline 100 & 0.97 \\
\hline
\end{tabular}

The calculated mean and standard deviation of the built-in voltages were $0.97 \mathrm{~V}$ and $0.01 \mathrm{~V}$ respectively.

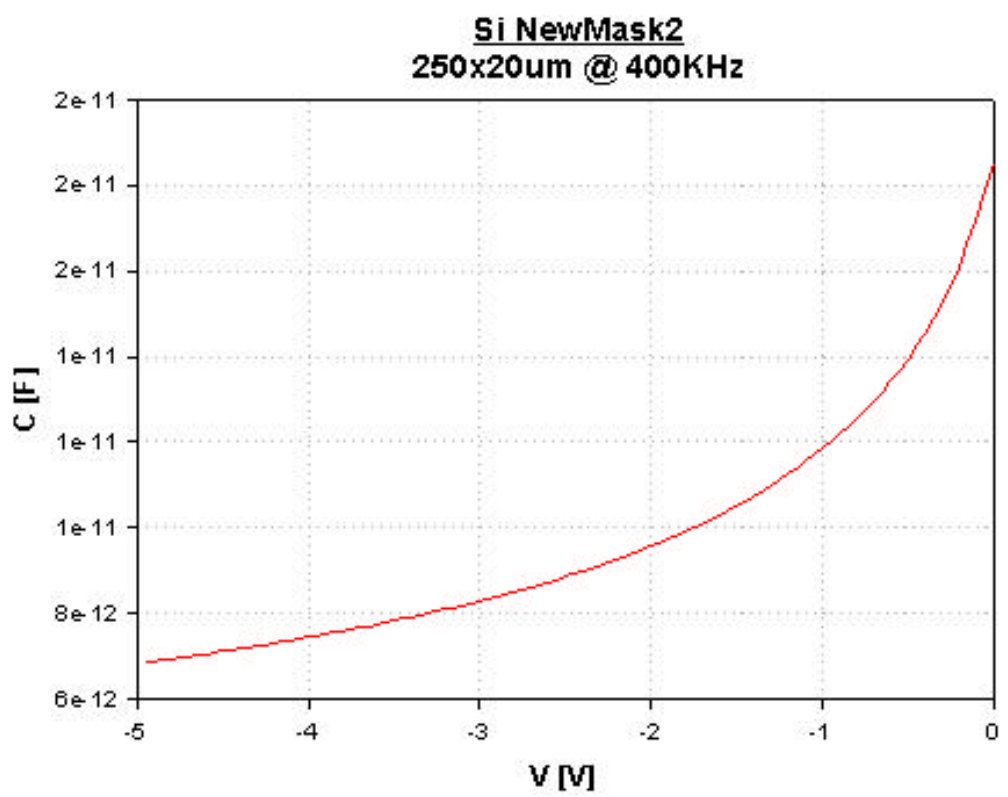

Figure 4.8: C-V of a $250 \mu \mathrm{m}$ dot with a $20 \mu \mathrm{m}$ of separation on $\mathrm{Si}$ at $400 \mathrm{KHz}$. 


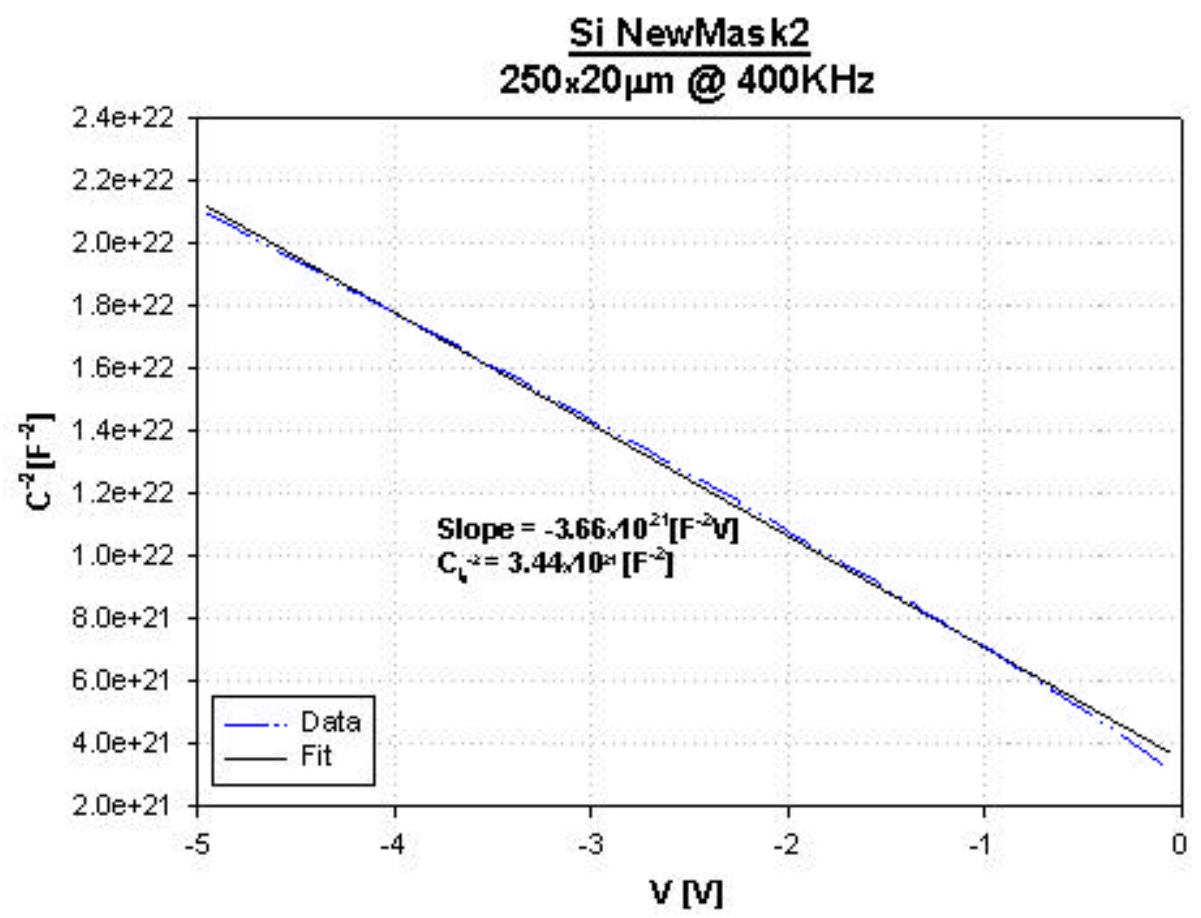

Figure 4.9: $\mathrm{C}^{-2} v s . \mathrm{V}$ of a $250 \mu \mathrm{m}$ dot with a $20 \mu \mathrm{m}$ of separation on $\mathrm{Si}$ at $400 \mathrm{KHz}$.

\subsubsection{CARRIER CONCENTRATION}

The first step in calculating the carrier concentration of a material is to assess whether or not there are interface-trapped charges present on it. As described on section 2.3.1.1 the way of doing this is by plotting the $\mathrm{C}-\mathrm{V}$ curve of the junction at different frequencies and look for a shift in the roll-off of the capacitance. A plot of $\mathrm{C}-\mathrm{V}$ at different frequencies is shown on figure 4.10. It is clearly seen on figure 4.10 that there is a shift in the roll-off of capacitance and this is a good indication that interface-trapped states are present in this sample. The plot only includes the minimum frequency that the equipment can use, and the maximum frequency at which the shift is not noticeable any longer. As described in Chapter 2, the way of getting around this problem is by taking the measurement at high frequencies so that these states cannot follow the test signal swing and not interfere in the capacitance reading. As it can be seen on figure 4.10 the surface states contribute for an additional potential to the built-in voltage, and this can be calculated by subtracting the theoretical $\left(0.57 \mathrm{~V}\right.$ - section 3.2) value from the experimental value of $\mathrm{V}_{\mathrm{bi}}$. In this case, this additional potential was calculated to be $0.42 \mathrm{~V}$. 


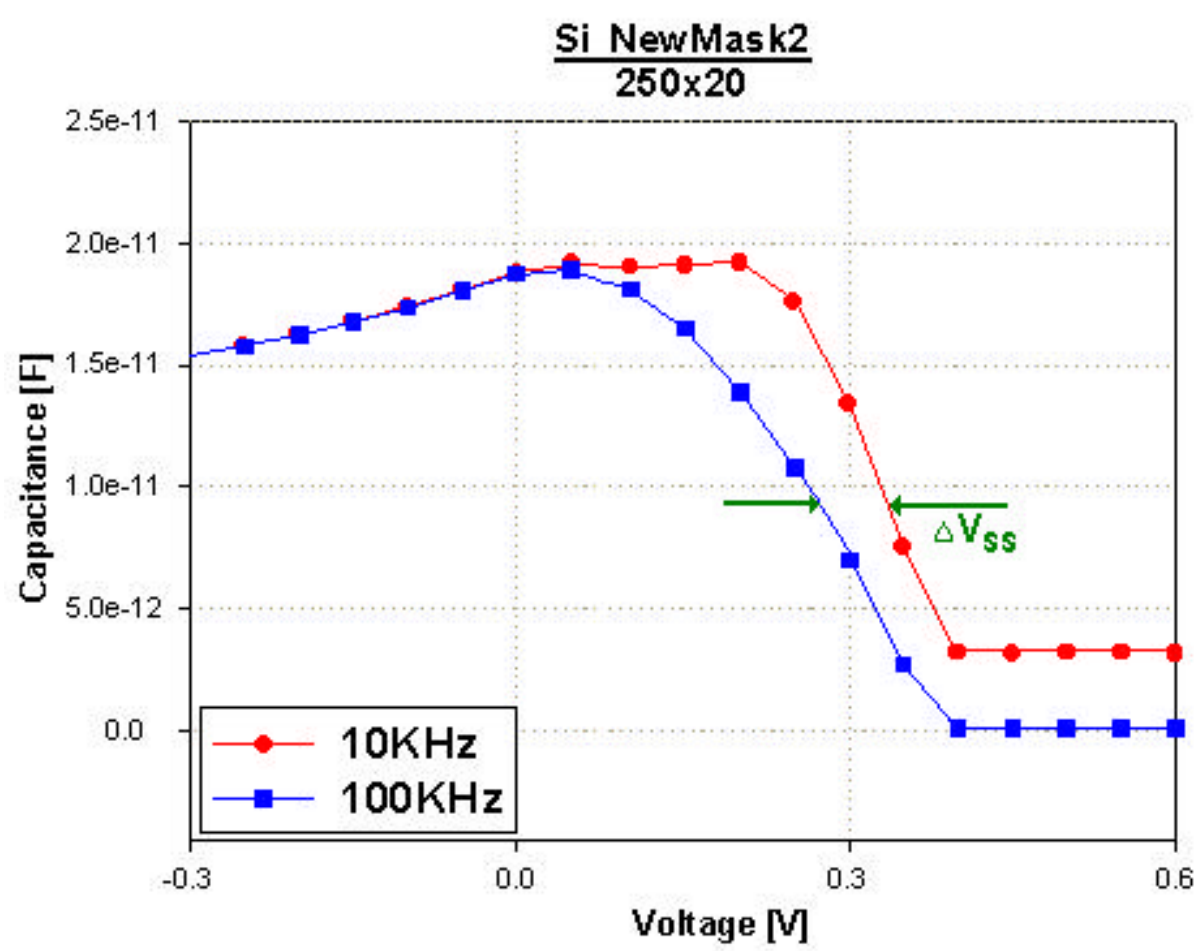

Figure 4.10: $\mathrm{C}-\mathrm{V}$ curve of a contact on $\mathrm{Si}$ at different test frequencies.

Another point that has to be observed is the roll-off of the carrier concentration with frequency. As stated on Chapter 2, and shown in figure 2.23, the carrier concentration has its ideal value at low frequencies and, due to the presence of elements such as the series resistance, gets smaller as the frequency increases.

The carrier concentration at each frequency is calculated as explained in Chapter 2. First a raw $\mathrm{C}-\mathrm{V}$ curve is measured. Then, $\mathrm{C}^{2}$ vs. $\mathrm{V}$ is plotted and its slope and intercept are calculated. The plots of the raw $\mathrm{C}-\mathrm{V}$ and $\mathrm{C}^{2} v s . \mathrm{V}$ at a specific frequency are the same as shown on figures 4.8 and 4.9. Using these values in equation 2.19b, a program (appendix C) calculates the carrier concentration. Applying this methodology, a typical contact $(250 \mu \mathrm{m}$ with $20 \mu \mathrm{m}$ of separation) was measured, and the carrier concentration of the material was extracted at different frequencies. Table 4.5 shows the values found for the carrier concentration. A plot of these values is shown on figure 4.11 . 
Table 4.5: Carrier concentration of $\mathrm{Si}$ at different frequencies.

\begin{tabular}{|c|c|}
\hline Frequency [Hz] & Carrier Conc. $\mathbf{x 1 0}^{\mathbf{1 4}}\left[\mathbf{c m}^{\mathbf{- 3}}\right]$ \\
\hline $10 \mathrm{~K}$ & 8.50 \\
\hline $20 \mathrm{~K}$ & 8.63 \\
\hline $40 \mathrm{~K}$ & 8.42 \\
\hline $100 \mathrm{~K}$ & 8.44 \\
\hline $200 \mathrm{~K}$ & 8.56 \\
\hline $400 \mathrm{~K}$ & 8.42 \\
\hline $1 \mathrm{M}$ & 8.33 \\
\hline $2 \mathrm{M}$ & 7.87 \\
\hline $4 \mathrm{M}$ & 7.02 \\
\hline
\end{tabular}

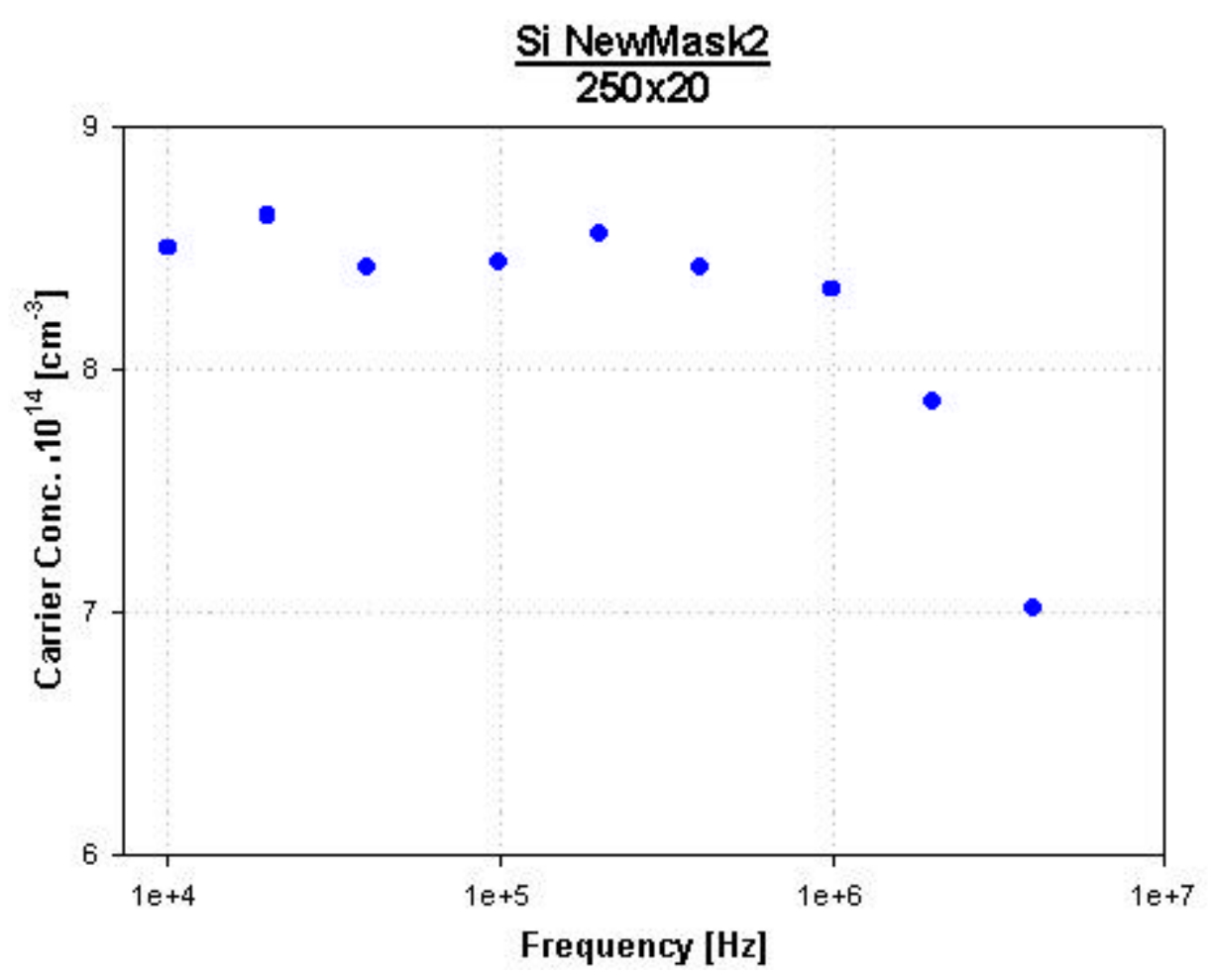

Figure 4.11: Plot of carrier concentration of $\mathrm{Si}$ at different frequencies. 
From figure 4.11 one can see that the values of the carrier concentration that are in the flat region of the curve are those between $10 \mathrm{KHz}$ and $400 \mathrm{KHz}$. The mean and standard deviation for the values of the carrier concentration between these frequencies are $8.50 \times 10^{14} \mathrm{~cm}^{-3}$ and $0.078 \times 10^{14} \mathrm{~cm}^{-3}$. A tradeoff appears when one compares the condition imposed by the decay of the carrier concentration with the requirement of a high-frequency measurement imposed by the presence of interface-trapped states. Also, one should consider the behavior of the loss tangent within the frequency range. It was shown on figure 4.5 that the capacitance reading has lowest losses at frequencies near $400 \mathrm{KHz}$. A more accurate way of calculating the carrier concentration should incorporate all these effects. In other words, the carrier concentration can be calculated at a point of frequency where the loss tangent is small, the carrier concentration lays on a flat portion of its curve versus frequency, and at sufficiently high frequency such that possible interface-trapped charges cannot follow the test signal swing. Therefore, the point at which the measurement should produce the best reading is at $400 \mathrm{KHz}$, and the value of the carrier concentration calculated at this frequency was $8.42 \times 10^{14} \mathrm{~cm}^{-3}$ as shown on table 4.5 . This value does not differ much from the value found by calculating the average of the values of the carrier concentration between $10 \mathrm{KHz}$ and $400 \mathrm{KHz}$. Also, as stated before, the nominal value of the carrier concentration determined by the manufacturer is between $6.3 \times 10^{14} \mathrm{~cm}^{-3}$ and $10^{15} \mathrm{~cm}^{-3}$. Therefore, the measurement produced a result very close to what was expected, which indicates a good accuracy in the procedure.

\subsection{G aN 9906E}

GaN9906E was the first sample of gallium nitride to be analyzed. Hall measurements (Appendix F) returned a resistivity of $10.838 \Omega-\mathrm{cm}$, and carrier concentration of $2.262 \times 10^{15} \mathrm{~cm}^{-3}$. The sample was cleaned and prepared as described in section 3.3. The Ohmic contact was aluminum, and the Schottky contact was platinum. The second mask design (fig. 3.2) was used to pattern the contacts. 


\subsubsection{RESISTANCE MEASUREMENTS}

The first measurement performed on this sample was an I-V analysis of four Ohmic contacts to extract the sheet resistance, and the specific contact resistance of the material. The four contacts measured were composed of Ohmic rings and dots. All the rings were $100 \mu \mathrm{m}$ wide separated by $40 \mu \mathrm{m}$ from the dots. The radii of the dots were $250 \mu \mathrm{m}, 200 \mu \mathrm{m}, 150 \mu \mathrm{m}, 100 \mu \mathrm{m}$. I$\mathrm{V}$ curves were taken for each of these structures, and the total resistances were then extracted directly from these curves by a fitting routine over the linear range of the curve. Figure 4.12 shows the I-V measured curves, and the total measured resistances are shown on table 4.6.

Table 4.6: Total resistance versus radius of five Ohmic contacts on GaN9906E.

\begin{tabular}{|c|c|}
\hline Radius $[\mu \mathrm{m}]$ & Total Resistance $[\Omega]$ \\
\hline 250 & $1.64 \mathrm{k}$ \\
\hline 200 & $1.83 \mathrm{k}$ \\
\hline 150 & $2.93 \mathrm{k}$ \\
\hline 100 & $4.71 \mathrm{k}$ \\
\hline
\end{tabular}

The values of resistance from table 4.6 were then fit with equation 2.35 to find the sheet resistance and the specific contact resistance. A plot of this fitting is shown on figure 4.13. The values found for the sheet resistance and specific contact resistance were $11.5 \mathrm{~K} \Omega$ and $1.6 \Omega-\mathrm{cm}^{2}$ respectively. The constant $\mathrm{L}_{\mathrm{T}}$ in this case was $118 \mu \mathrm{m}$. Since it is approximately 2 times smaller than the dot radius $(250 \mu \mathrm{m})$, equation 2.38 has to be used with caution. In equation 2.35 , the quotient of the I functions in this case is 1.4 , and the quotient of the $\mathrm{K}$ functions is 0.82 . The error in this situation can be high, and therefore, at these high resistances, a precise measurement of the sheet resistance and specific contact resistance, using this technique, cannot be achieved. 


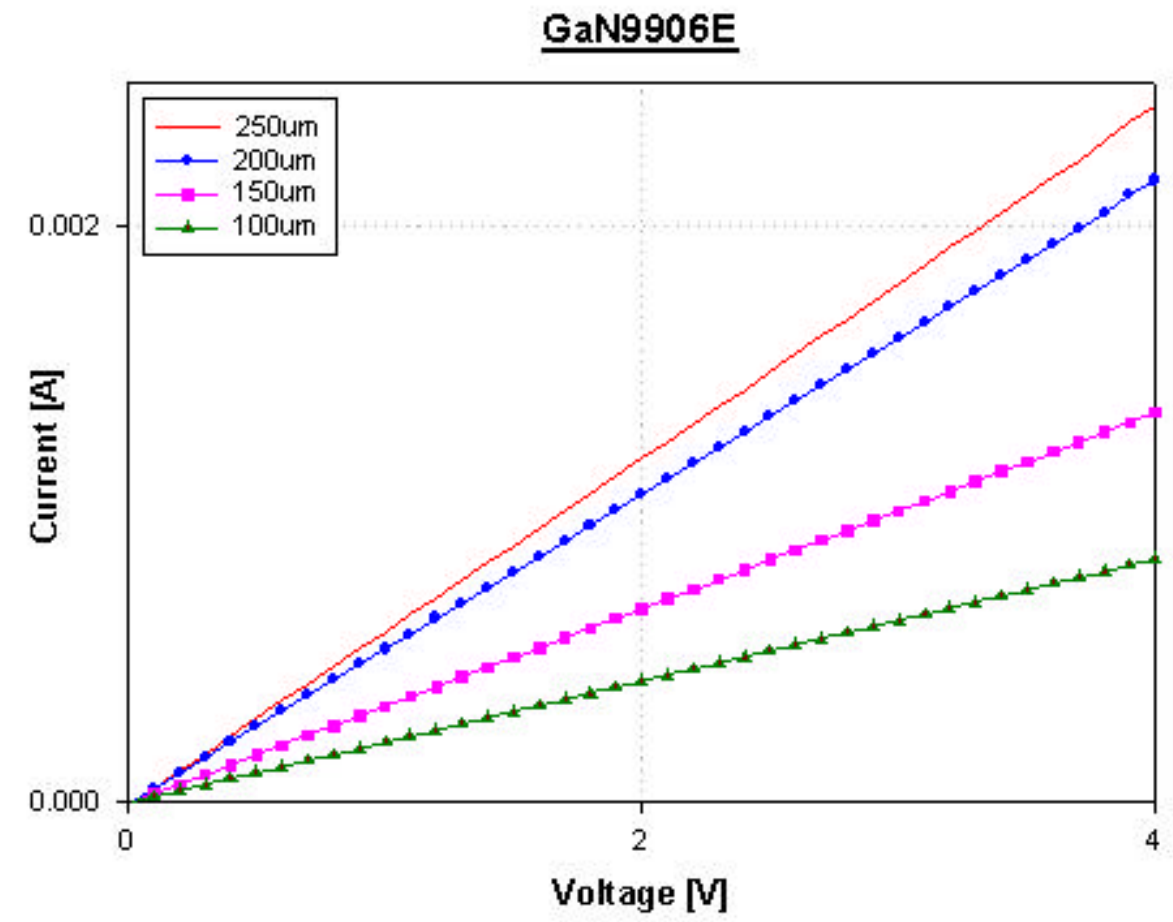

Figure 4.12: I-V curves of four Ohmic contacts on GaN9906E.

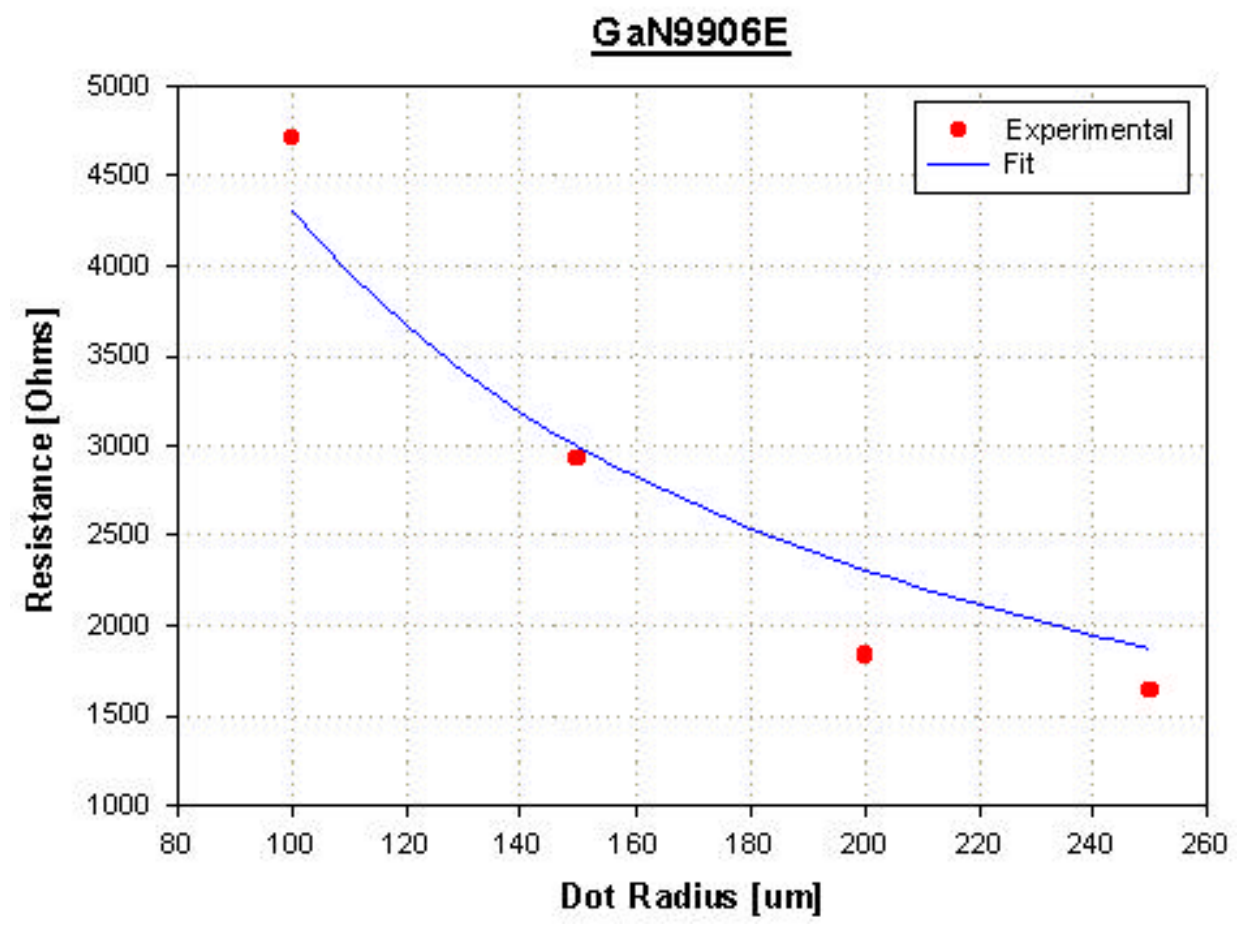

Figure 4.13: Fitting of resistance versus dot radius on GaN9906E. 


\subsubsection{SCHOTTKY MEASUREMENTS}

Following the I-V measurements on Ohmic contacts, a second set of I-V measurements was taken on Schottky contacts to verify their proper behavior. Four contacts were measured. All of them had a $100 \mu \mathrm{m}$ wide Ohmic ring, a Schottky dot with $250 \mu \mathrm{m}$ or radius, and separations of $20 \mu \mathrm{m}, 50 \mu \mathrm{m}, 60 \mu \mathrm{m}$, and $80 \mu \mathrm{m}$ between the dot and the ring. The plots of the log $\mathrm{FV}$ and linear

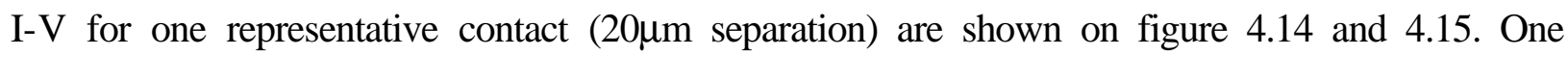
peculiar piece of information found was that the saturation current was much higher than for silicon, and also much higher than what is found on the literature for gallium nitride $\left(\mathrm{I}_{\mathrm{SAT}}<10^{-6} \mathrm{~A}\right)$ [15-17][19].

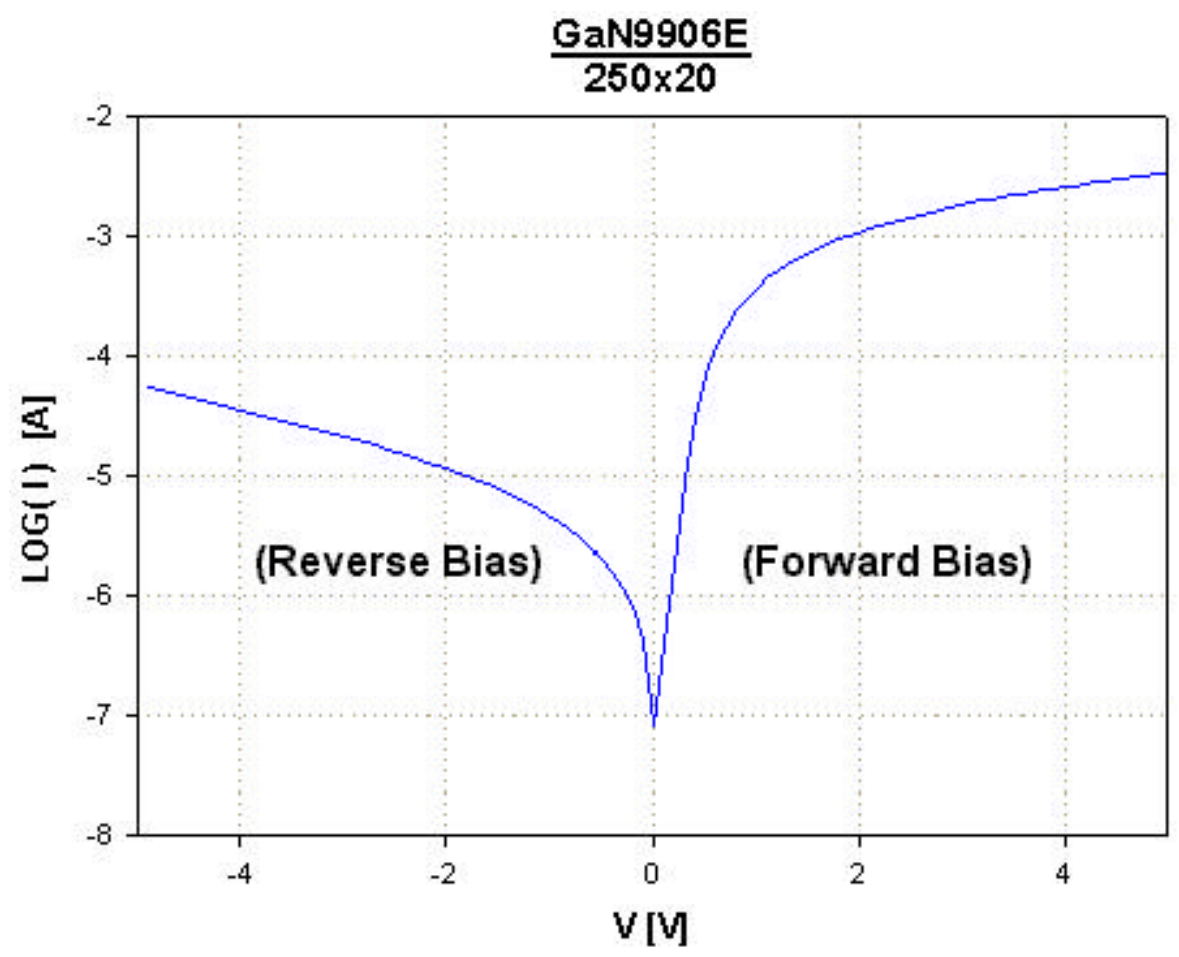

Figure 4.14: Log I-V plot of a $250 \mu \mathrm{m}$ dot with $20 \mu \mathrm{m}$ of separation on GaN9906E. 


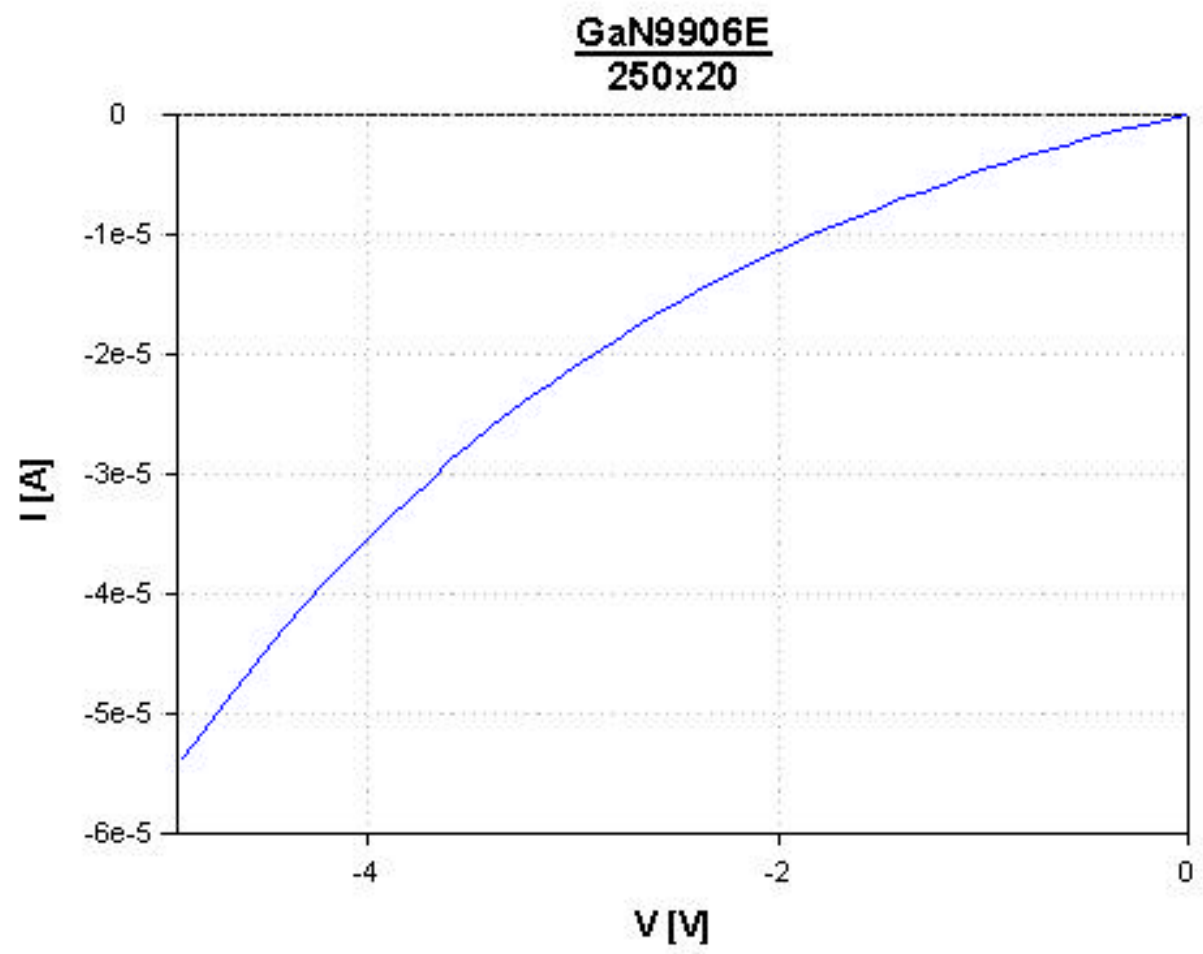

Figure 4.15: Saturation current of a $250 \mu \mathrm{m}$ dot with $20 \mu \mathrm{m}$ of separation on GaN9906E.

\subsubsection{LOSS TANGENT}

The dissipation factor was measured versus frequency at zero applied bias voltage. A representative plot, obtained from the same structure $(250 \mu \mathrm{m}$ dot with $20 \mu \mathrm{m}$ of separation between the dot and the ring), is shown in figure 4.16. The point where the loss tangent reaches its minimum is $20 \mathrm{KHz}$. At this frequency the dissipation factor is $0.312(>0.1)$.

\subsubsection{DEPLETION CAPACITANCE AT NO BIAS}

The plots of the measured capacitance and conductance as functions of the frequency of a representative contact $(250 \mu \mathrm{m}$ and $20 \mu \mathrm{m}$ of separation) are shown on figures 4.17 and 4.18 . It can be seen for this sample that points between $10 \mathrm{KHz}$ and $40 \mathrm{KHz}$ should produce the most reliable measurement. 


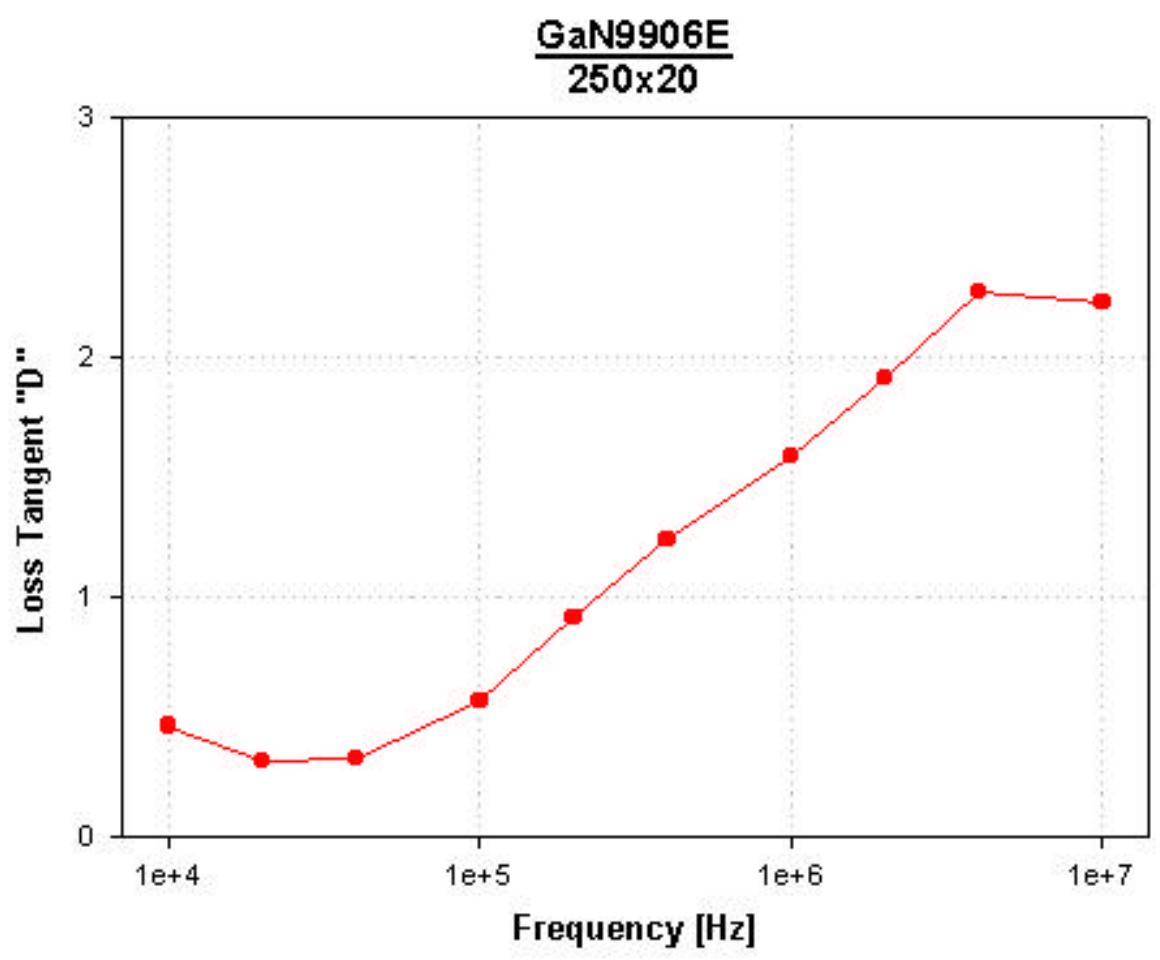

Figure 4.16: Loss tangent versus frequency of a $250 \mu \mathrm{m}$ dot with $20 \mu \mathrm{m}$ of separation on GaN9906E.

\subsubsection{BUILT-IN VOLTAGE}

Four contacts were measured. All contacts had a dot with $250 \mu \mathrm{m}$ of radius, but different separations between their dots and rings. The separations were $20 \mu \mathrm{m}, 50 \mu \mathrm{m}, 60 \mu \mathrm{m}$, and $80 \mu \mathrm{m}$. The best points in frequency determined for the built-in voltage measurement for each of these contacts are shown on table 4.7 .

Table 4.7: Points of lowest losses for the measured contacts on GaN9906E.

\begin{tabular}{|c|c|c|c|}
\hline Separation $[\mu \mathrm{m}]$ & Frequency $[\mathbf{H z}]$ & Loss tangent 'D' & $\mathbf{C}_{\mathbf{j o}}[\mathbf{p F}]$ \\
\hline 20 & $20 \mathrm{~K}$ & 0.31 & 204.00 \\
\hline 50 & $40 \mathrm{~K}$ & 0.73 & 160.40 \\
\hline 60 & $20 \mathrm{~K}$ & 0.3 & 193.10 \\
\hline 80 & $40 \mathrm{~K}$ & 0.6 & 176.75 \\
\hline
\end{tabular}




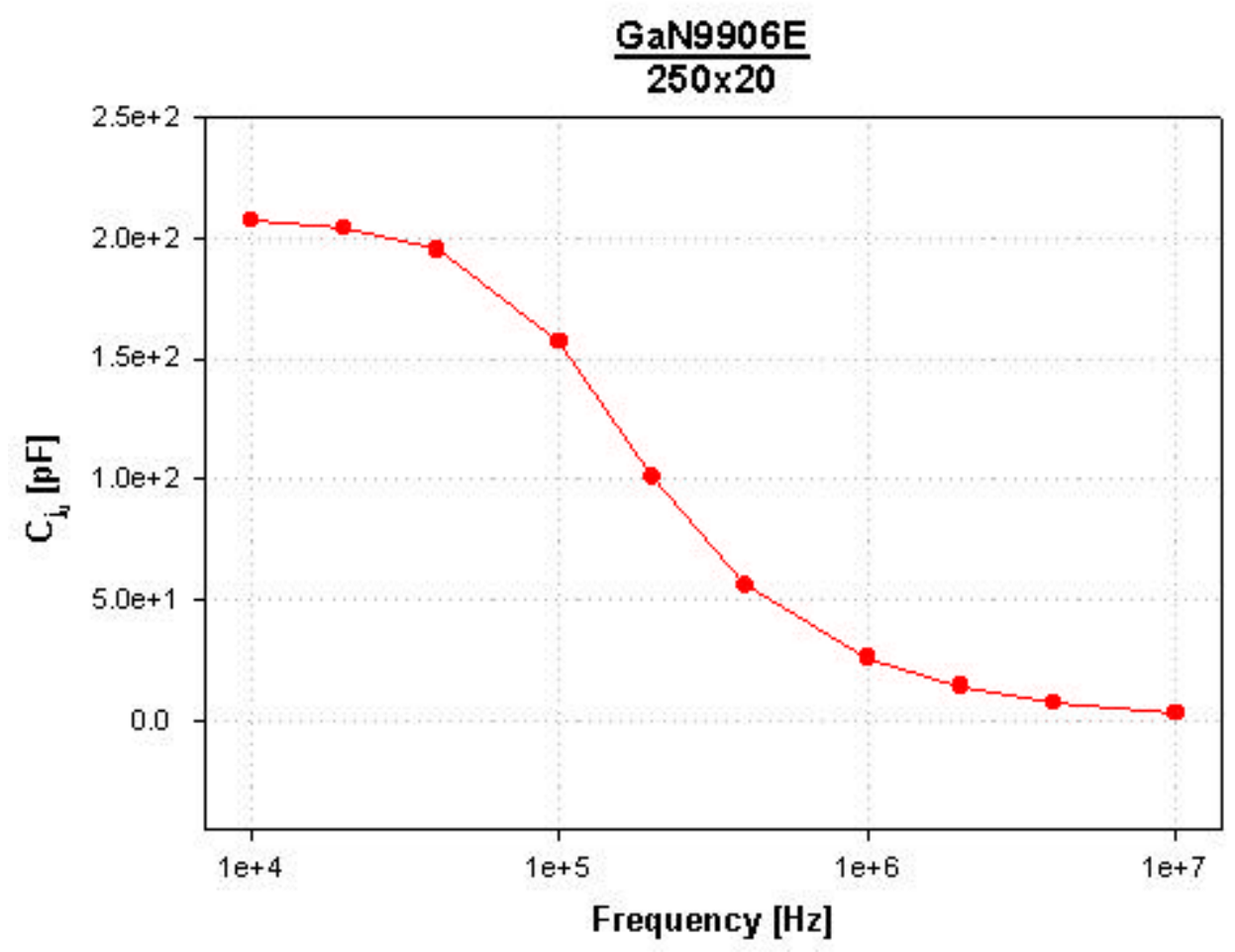

Figure 4.17: Junction capacitance of a $250 \mu \mathrm{m}$ dot with $20 \mu \mathrm{m}$ of separation at zero bias on GaN9906E

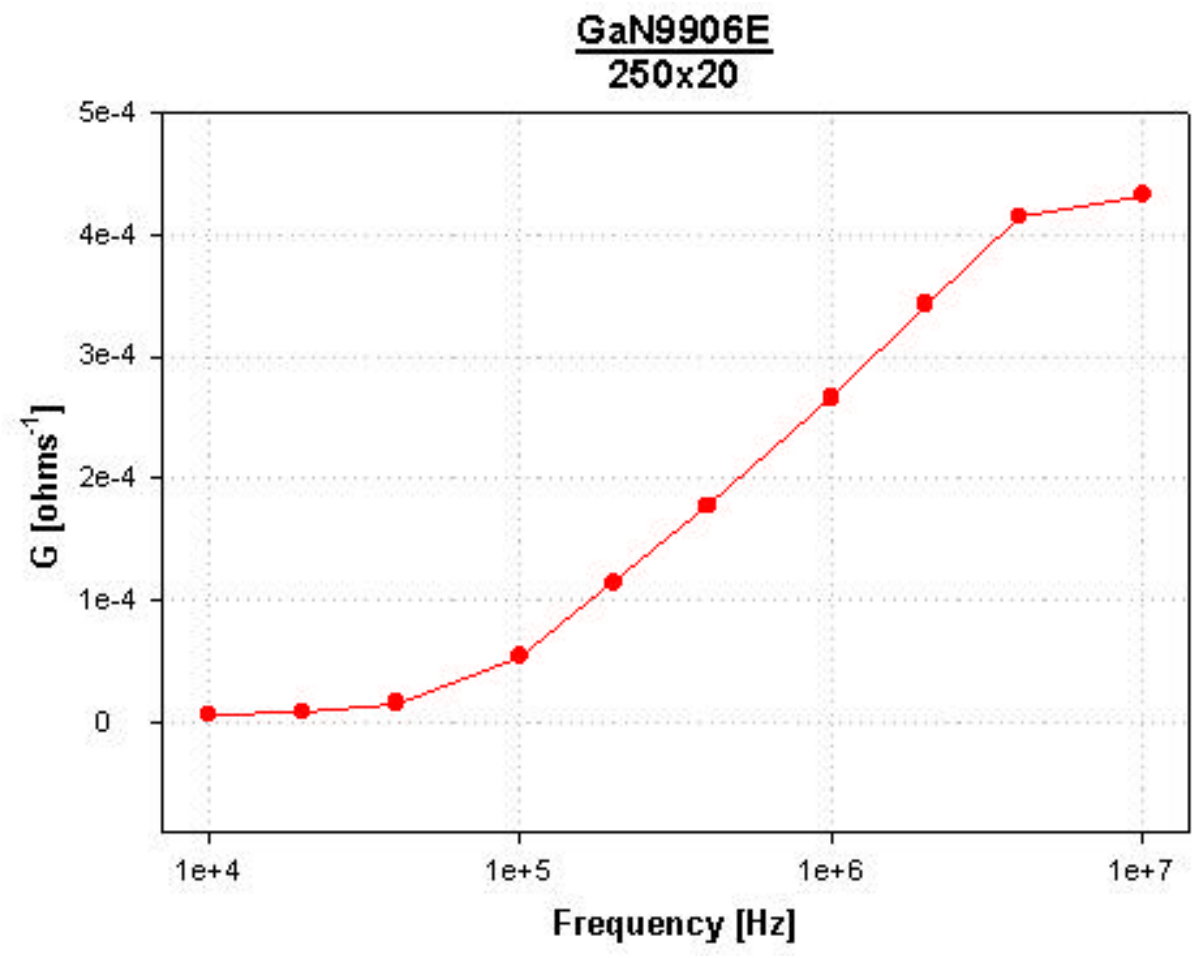

Figure 4.18: Junction conductance of a $250 \mu \mathrm{m}$ dot with $20 \mu \mathrm{m}$ of separation at zero bias on GaN9906E. 
The calculated mean and standard deviation of the loss tangent were 0.19 and 0.05 respectively. The calculated mean and standard deviation for the measured capacitance at no bias were $183.6 \mathrm{pF}$ and $16.52 \mathrm{pF}$ respectively. Based on the results from table 4.7, C-V curves were taken at those specific frequencies. The $\mathrm{C}-\mathrm{V}$ curve of a representative contact $(250 \mu \mathrm{m}$ dot with $20 \mu \mathrm{m}$ of separation) is shown on figure 4.19. The plot of $\mathrm{C}^{-2} v s$. V obtained from the same contact is shown on figure 4.20. The values obtained for the slope and intercept for each of the C ${ }^{2}$ vs. V curves are shown on table 4.8. Using the values of table 4.8, a program (appendix C) applies equation $2.20 \mathrm{~b}$ to calculate the built-in voltage. These results are found on table 4.9.

Table 4.8: Slope and intercept of $\mathrm{C}^{-2} v s$. V curves of four contacts on GaN9906E.

\begin{tabular}{|c|c|c|}
\hline Separation $[\mu \mathbf{m}]$ & Slope $\mathbf{x} \mathbf{1 0}^{19}\left[\mathbf{F}^{-2} \mathbf{V}^{-1}\right]$ & Intercept $_{\mathbf{x}} \mathbf{1 0}^{19}\left[\mathbf{F}^{-2}\right]$ \\
\hline 20 & -3.78 & 2.36 \\
\hline 50 & -6.01 & 3.65 \\
\hline 60 & -3.97 & 2.60 \\
\hline 100 & -5.00 & 2.97 \\
\hline
\end{tabular}

Table 4.9: Calculated built-in voltages for the measured contacts on GaN9906E.

\begin{tabular}{|c|c|}
\hline Separation $[\mu \mathrm{m}]$ & Built-in Voltage [V] \\
\hline 20 & 0.65 \\
\hline 50 & 0.63 \\
\hline 60 & 0.68 \\
\hline 100 & 0.62 \\
\hline
\end{tabular}




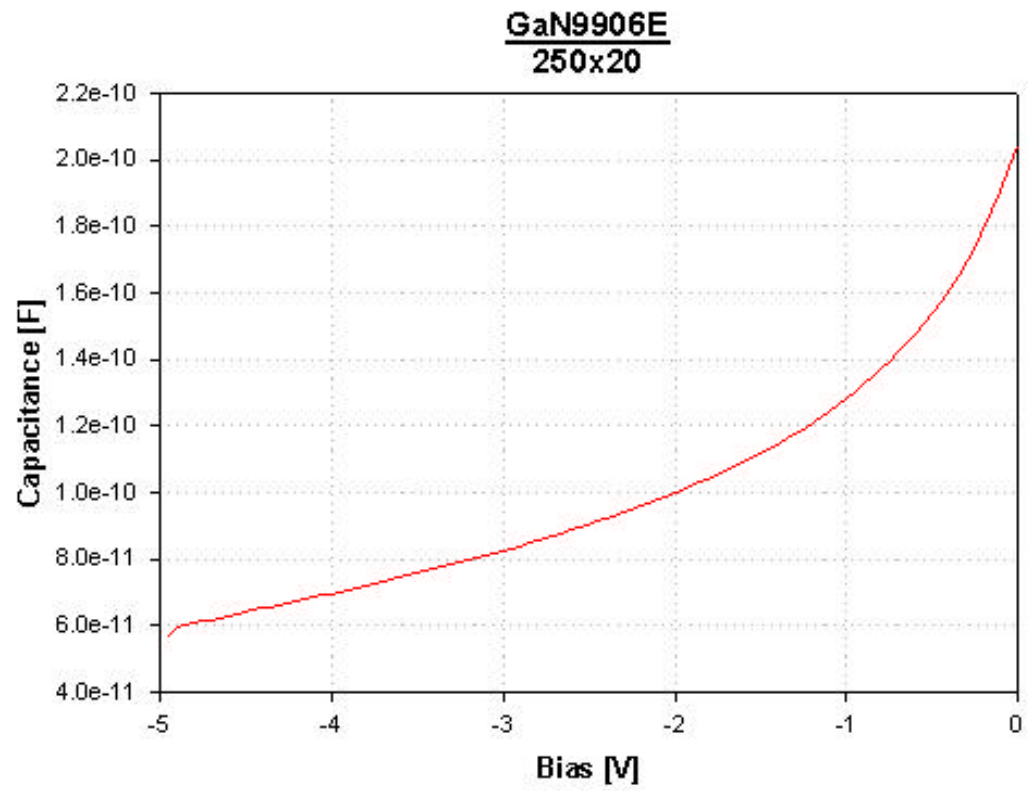

Figure 4.19: $\mathrm{C}-\mathrm{V}$ of a $250 \mu \mathrm{m}$ dot with a $20 \mu \mathrm{m}$ of separation at $20 \mathrm{KHz}$ on GaN9906E.

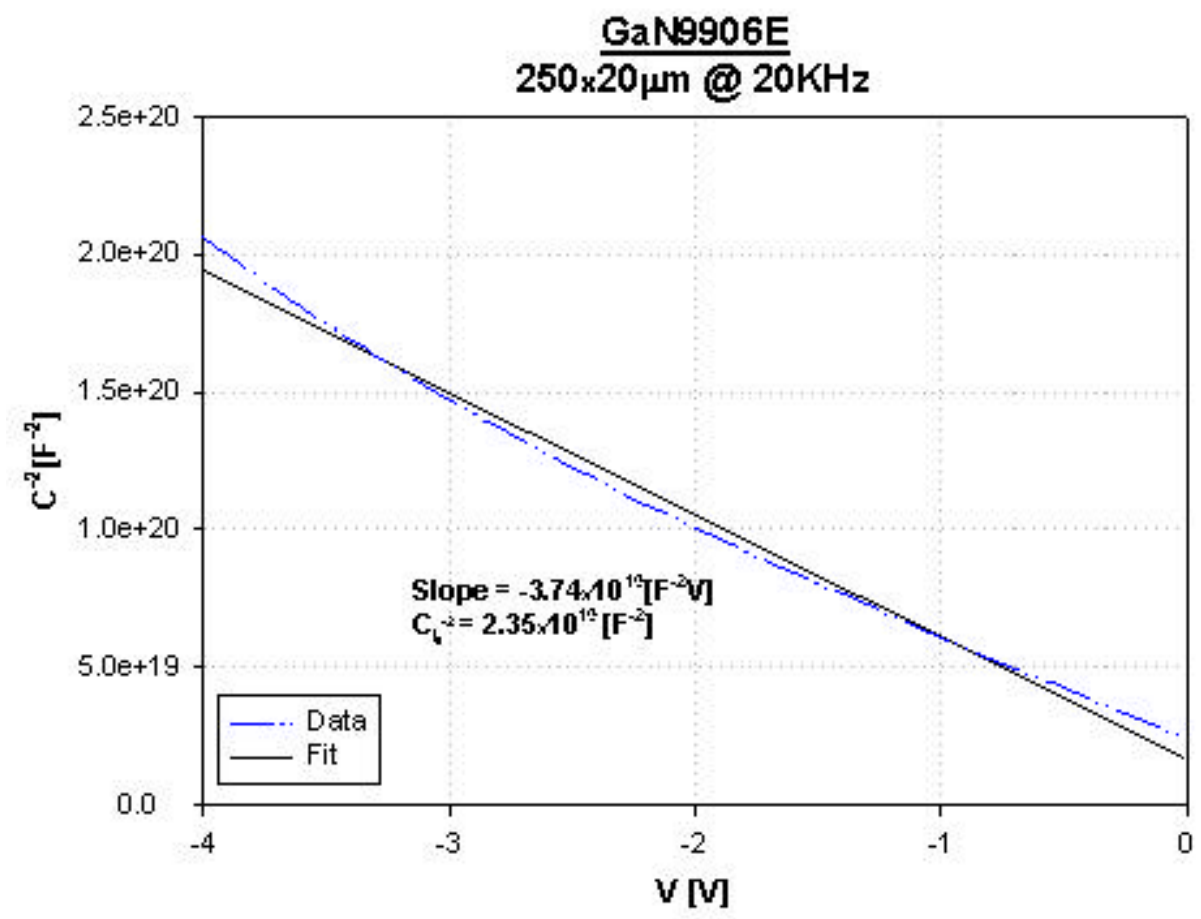

Figure 4.20: $\mathrm{C}^{-2} v s . \mathrm{V}$ of a $250 \mu \mathrm{m}$ dot with a $20 \mu \mathrm{m}$ of separation at $20 \mathrm{KHz}$ on GaN9906E. 
The calculated mean and standard deviation of the built-in voltage were $0.645 \mathrm{~V}$ and $0.023 \mathrm{~V}$ respectively. However, some problems were noticed with this sample of gallium nitride. These problems include the product rG bigger than the unity, high saturation current, and dissipation factor bigger than 0.1. Therefore, a correction factor (Eq. 2.23b) was used to calculate the real value of the built-in voltage. Using the series resistance given on table 4.6 and the conductance as shown on figure 4.18 the values of the built-in voltage were corrected. This new set of values is shown on table 4.10 .

Table 4.10: Corrected built-in voltages for contacts on GaN9906E.

\begin{tabular}{|c|c|}
\hline Separation $[\mu \mathbf{m}]$ & Built-in Voltage [V] \\
\hline 20 & 0.68 \\
\hline 50 & 0.76 \\
\hline 60 & 0.71 \\
\hline 100 & 0.74 \\
\hline
\end{tabular}

The new mean and standard deviation of the built-in voltage were $0.72 \mathrm{~V}$ and $0.03 \mathrm{~V}$ respectively. The theoretical value of the built-in voltage, as calculated in section 3.3.1, is $1.55 \mathrm{~V}$. This indicates a discrepancy between the theoretical and experimental value. One proposed possible explanation that can be considered is that since the saturation current is so high for this sample, one additional capacitance due to minority carriers can be present, and this could be interfering in the measurement.

\subsubsection{CARRIER CONCENTRATION}

A plot of $\mathrm{C}-\mathrm{V}$ at different frequencies is shown on figure 4.21. It is clearly seen on figure 4.21 that there is a shift in the roll-off of capacitance and this is a good indication that interfacetrapped states are present in this sample. The plot only includes the minimum frequency that the equipment can use, and the maximum frequency at which the shift is not noticeable any longer. The density of interface-trapped charges can be estimated by several methods [6][24], however it is suggested as a future study for gallium nitride. 
A typical contact $(250 \mu \mathrm{m}$ with $20 \mu \mathrm{m}$ of separation) was measured, and the carrier concentration of the material was extracted at different frequencies. Table 4.11 shows the values found for the carrier concentration. A plot of these values is shown on figure 4.22.

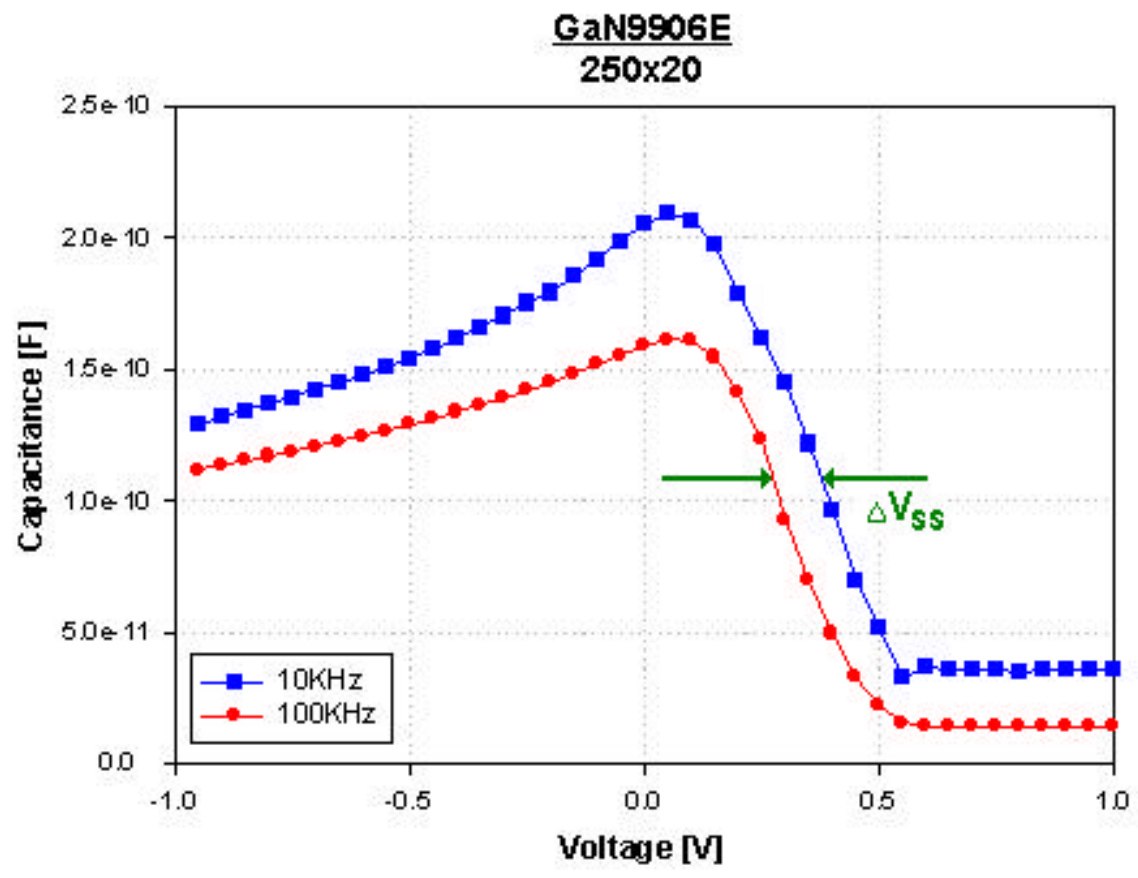

Figure 4.21: C-V curve of a contact on GaN9906E at different test frequencies. 


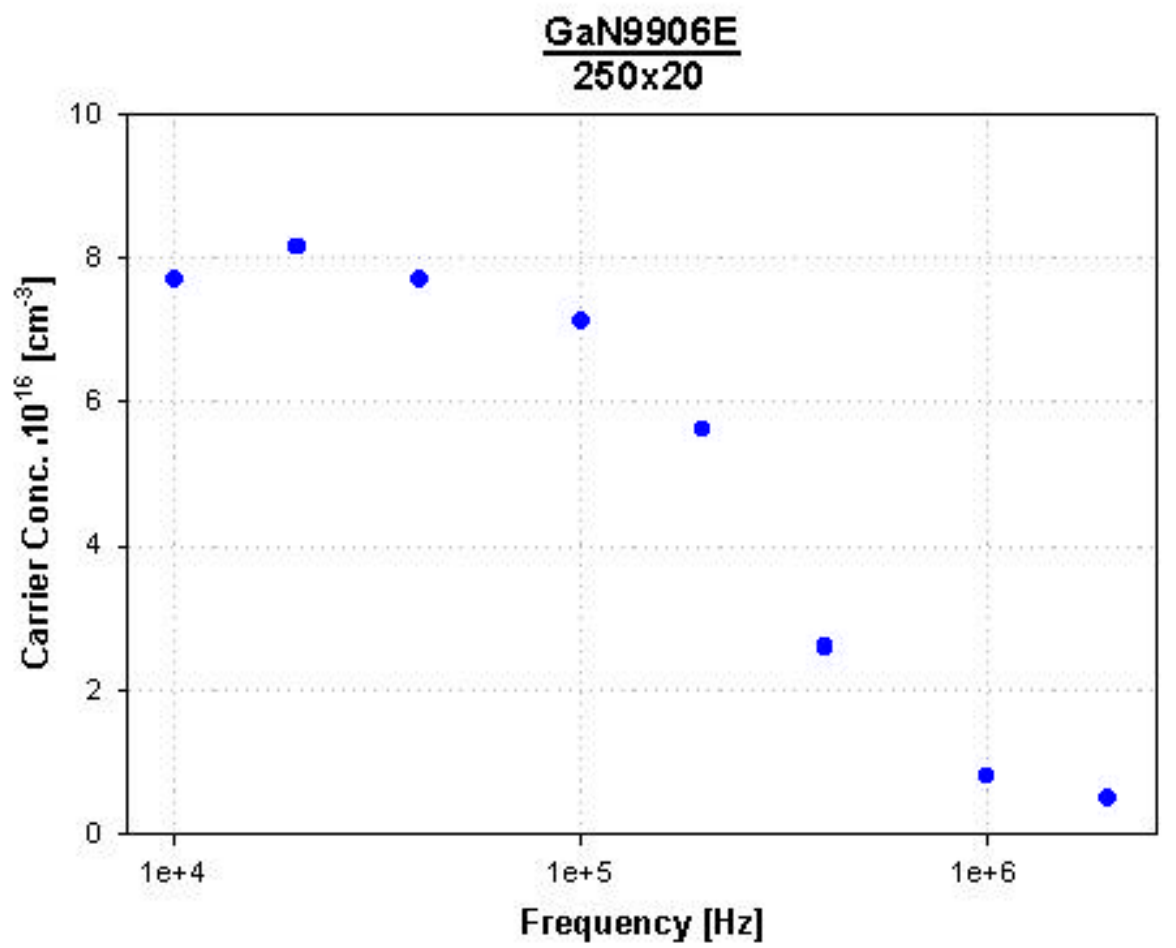

Figure 4.22: Plot of carrier concentration of GaN9906E at different frequencies.

Table 4.11: Carrier concentration of GaN9906E at different frequencies.

\begin{tabular}{|c|c|}
\hline Frequency $[\mathbf{H z}]$ & Carrier Conc. $\mathbf{x 1 0}^{\mathbf{1 6}}\left[\mathbf{c m}^{\mathbf{3}}\right]$ \\
\hline $10 \mathrm{~K}$ & 7.69 \\
\hline $20 \mathrm{~K}$ & 8.15 \\
\hline $40 \mathrm{~K}$ & 7.7 \\
\hline $100 \mathrm{~K}$ & 7.12 \\
\hline $200 \mathrm{~K}$ & 5.62 \\
\hline $400 \mathrm{~K}$ & 2.58 \\
\hline $1 \mathrm{M}$ & 0.79 \\
\hline $2 \mathrm{M}$ & 0.48 \\
\hline
\end{tabular}

From figure 4.22 one can see that the values of the carrier concentration that are not very dependent on frequency effects are those between $10 \mathrm{KHz}$ and $40 \mathrm{KHz}$. The mean and standard deviation for the values of the carrier concentration between these frequencies are $7.85 \times 10^{16} \mathrm{~cm}^{-3}$ and $0.21 \times 10^{16} \mathrm{~cm}^{-3}$. It was shown in figure 4.16 that the capacitance reading has fewer losses at frequencies near $20 \mathrm{KHz}$. The value of the carrier concentration calculated at this frequency was $8.15 \times 10^{16} \mathrm{~cm}^{-3}$ as shown in table 4.11 . 
Since the product $\mathrm{rG}$ is comparable to the unity in this sample, a correction factor (Eq. 2.22c) was used to adjust the values of the carrier concentration for the points that are in the flat region of the curve of carrier concentration vs. frequency. The corrections were made using the values of the series resistance given in table 4.5 and the conductance as shown in figure 4.18. The adjusted values of the carrier concentration are shown in table 4.12.

Table 4.12: Adjusted values of the carrier concentration for GaN9906E.

\begin{tabular}{|c|c|}
\hline Frequency [Hz] & Carrier Conc. $\mathbf{x 1 0}^{\mathbf{1 6}}\left[\mathbf{c m}^{\mathbf{- 3}}\right]$ \\
\hline $10 \mathrm{~K}$ & 8.00 \\
\hline $20 \mathrm{~K}$ & 8.59 \\
\hline $40 \mathrm{~K}$ & 8.54 \\
\hline
\end{tabular}

The mean and the standard deviation of the new values of the carrier concentration were calculated to be $8.38 \times 10^{16} \mathrm{~cm}^{-3}$ and $0.267 \times 10^{16} \mathrm{~cm}^{-3}$ respectively. The carrier concentration found by Hall was $2.26 \times 10^{15} \mathrm{~cm}^{-3}$. This indicates a discrepancy between the two measurements. The same possible hypothesis that was suggested to explain the discrepancy found with the built-in voltage can be taken into account for the carrier concentration. Since the saturation current is very high for this sample, there may exist an additional capacitance that influence the measurement and therefore an accurate measurement of the carrier concentration at this high saturation current may not be achieved. Another point that can be analyzed is the width of the depletion layer. For this sample, using equation $2.14 \mathrm{~b}$ with the theoretical value of the built-in voltage, and the carrier concentration found by $\mathrm{C}-\mathrm{V}$, the width of the depletion layer is $0.14 \mu \mathrm{m}$ at zero bias, and $0.28 \mu \mathrm{m}$ at $-5 \mathrm{~V}$. If the same calculation is performed with the value of the carrier concentration obtained by Hall, the width of the depletion width is $0.45 \mu \mathrm{m}$ at zero bias, and $0.92 \mu \mathrm{m}$ at $-5 \mathrm{~V}$.

\subsection{G aN 9920}

One more sample of gallium nitride, GaN9920, was processed to verify the behavior observed with the previous gallium nitride sample. Hall measurements (Appendix F) returned a resistivity of $35.815 \Omega-\mathrm{cm}$, and carrier concentration of $1.253 \times 10^{15} \mathrm{~cm}^{-3}$. The sample was cleaned 
and prepared according to section 3.3. The Ohmic contact was aluminum, and the Schottky contact was platinum. The second mask design (fig. 3.2) was used to pattern the contacts.

\subsubsection{RESISTANCE MEASUREMENTS}

The first measurement performed on this sample was an I-V analysis of five Ohmic contacts to extract the sheet resistance, and the specific contact resistance of the material. The

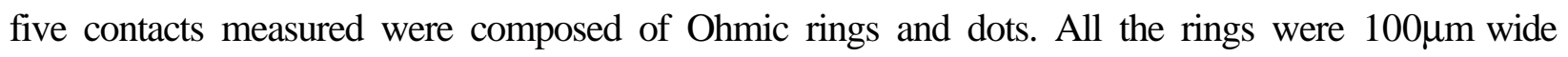
separated by $40 \mu \mathrm{m}$ from the dots. The radii of the dots were $250 \mu \mathrm{m}, 200 \mu \mathrm{m}, 150 \mu \mathrm{m}, 100 \mu \mathrm{m}$. I$\mathrm{V}$ curves were taken for each of these structures, and the total resistances were then extracted directly from these curves by a fitting routine over the linear range of the curve. Figure 4.23 shows the I-V measured curves, and the total measured resistances are shown in table 4.13.

Table 4.13: Total resistance versus radius of five Ohmic contacts on GaN9920.

\begin{tabular}{|c|c|}
\hline Radius $[\mu \mathbf{m}]$ & Total Resistance $[\Omega]$ \\
\hline 250 & $3.302 \mathrm{k}$ \\
\hline 200 & $4.613 \mathrm{k}$ \\
\hline 150 & $6.362 \mathrm{k}$ \\
\hline 100 & $9.376 \mathrm{k}$ \\
\hline 50 & $18.797 \mathrm{k}$ \\
\hline
\end{tabular}




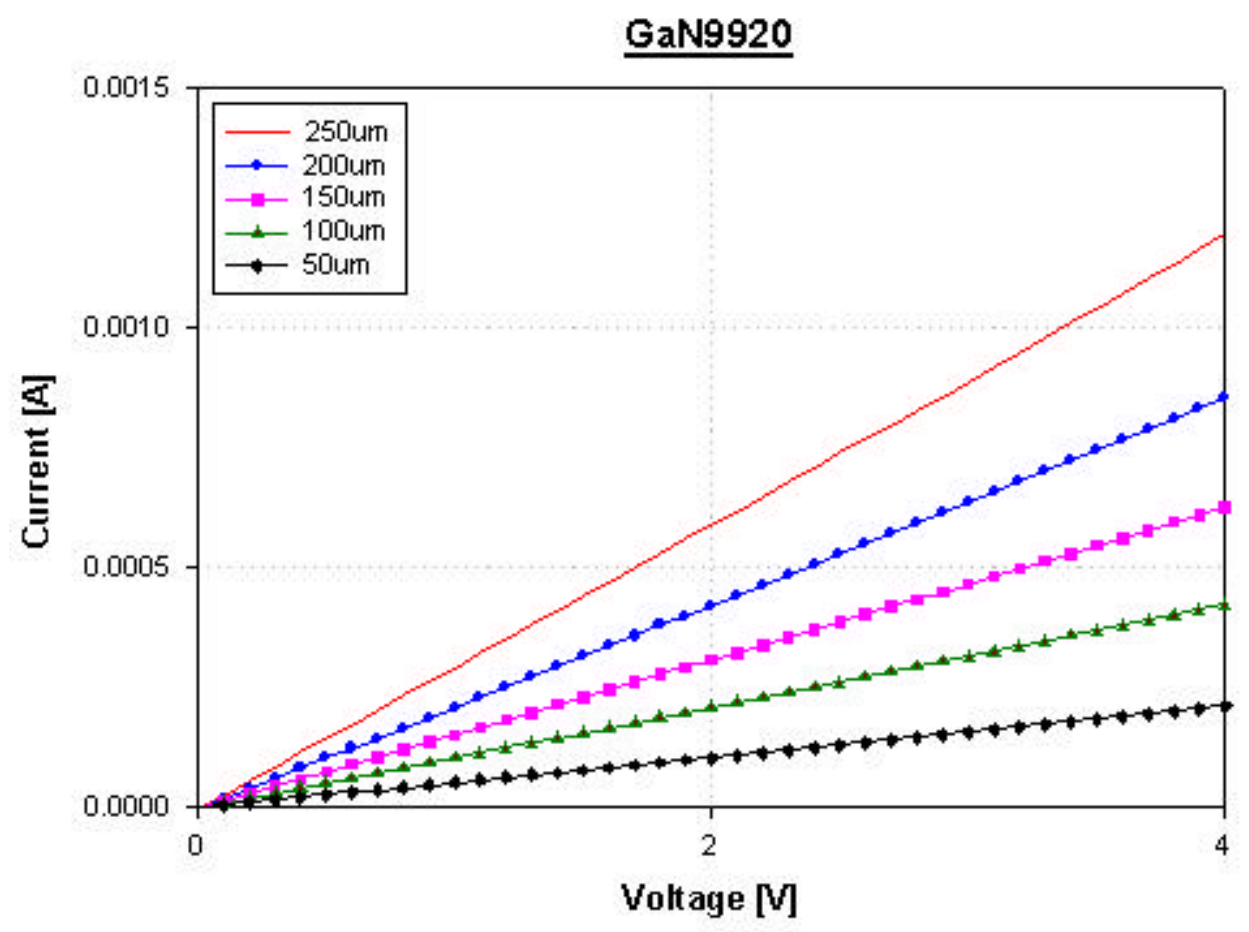

Figure 4.23: I-V curves of five Ohmic contacts on GaN9920.

The values of resistance from table 4.5 were then fit with equation 2.35 to find the sheet resistance and the specific contact resistance. A plot of this fitting is shown on figure 4.24. The values found for the sheet resistance and specific contact resistance were $8.92 \mathrm{~K} \Omega$ and $13.23 \Omega$ $\mathrm{cm}^{2}$ respectively. The constant $\mathrm{L}_{\mathrm{T}}$ in this case was $385 \mu \mathrm{m}$. Since it is approximately 1.5 times bigger than the dot radius $(250 \mu \mathrm{m})$, equation 2.38 has to be used with caution. In equation 2.35, the quotient of the I functions in this case is 3.15 , and the quotient of the $\mathrm{K}$ functions is 0.62 . The error in this situation can be high, and therefore, at these high resistances, a precise measurement of the sheet resistance and specific contact resistance, using this technique, cannot be achieved.

\subsubsection{SCHOTTKY MEASUREMENTS}

Following the I-V measurements on Ohmic contacts, a second set of $\mathrm{I}-\mathrm{V}$ measurements was taken on Schottky contacts to verify their proper behavior. Four contacts were measured. All of them had a $100 \mu \mathrm{m}$ wide Ohmic ring, a Schottky dot with $250 \mu \mathrm{m}$ or radius, and separations of 
$20 \mu \mathrm{m}, 50 \mu \mathrm{m}, 60 \mu \mathrm{m}$, and $80 \mu \mathrm{m}$ between the dot and the ring. The plots of the log $\mathrm{FV}$ and linear

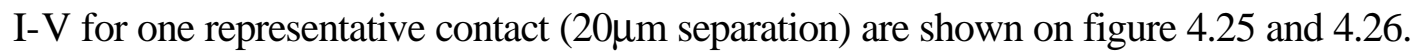

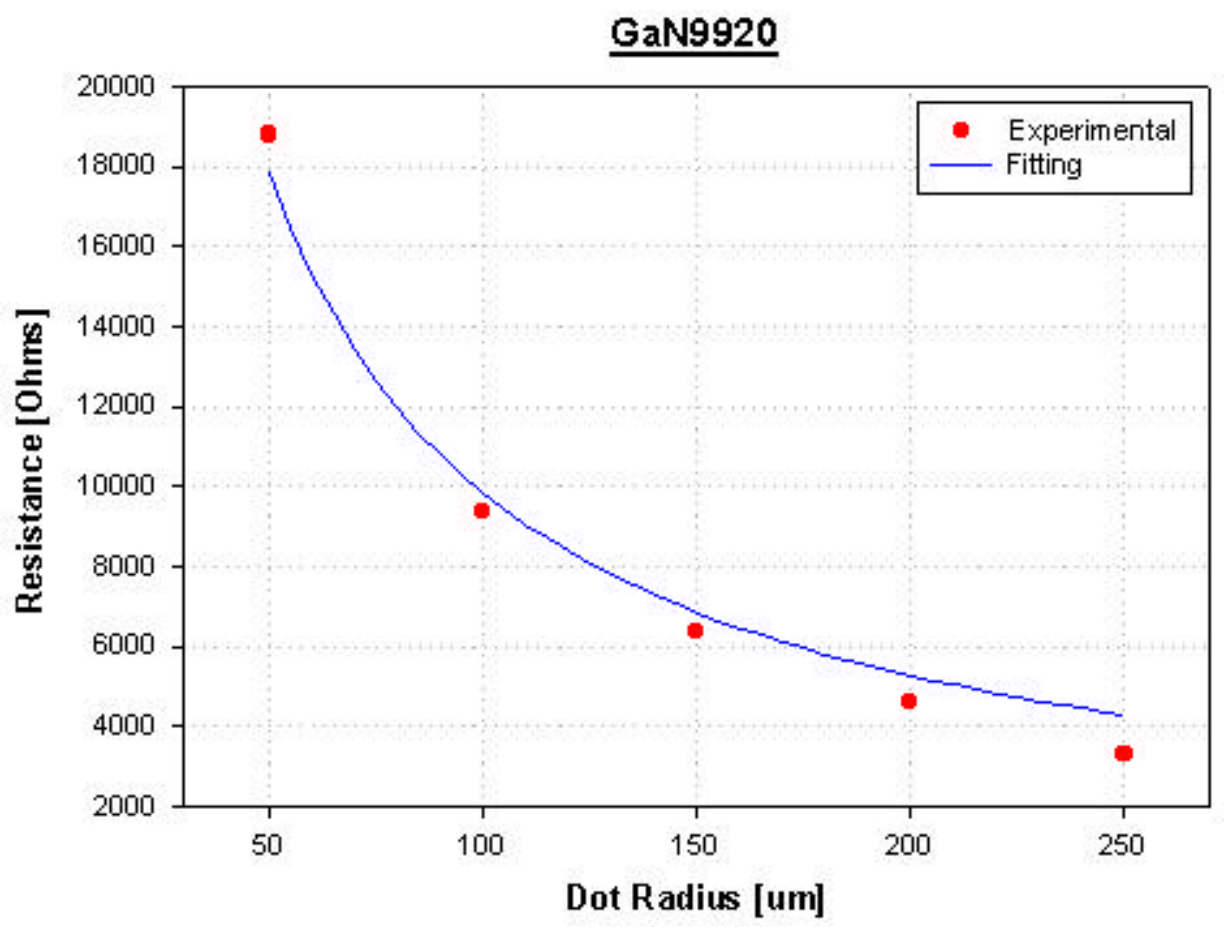

Figure 4.24: Fitting of resistance versus dot radius on GaN9920. 


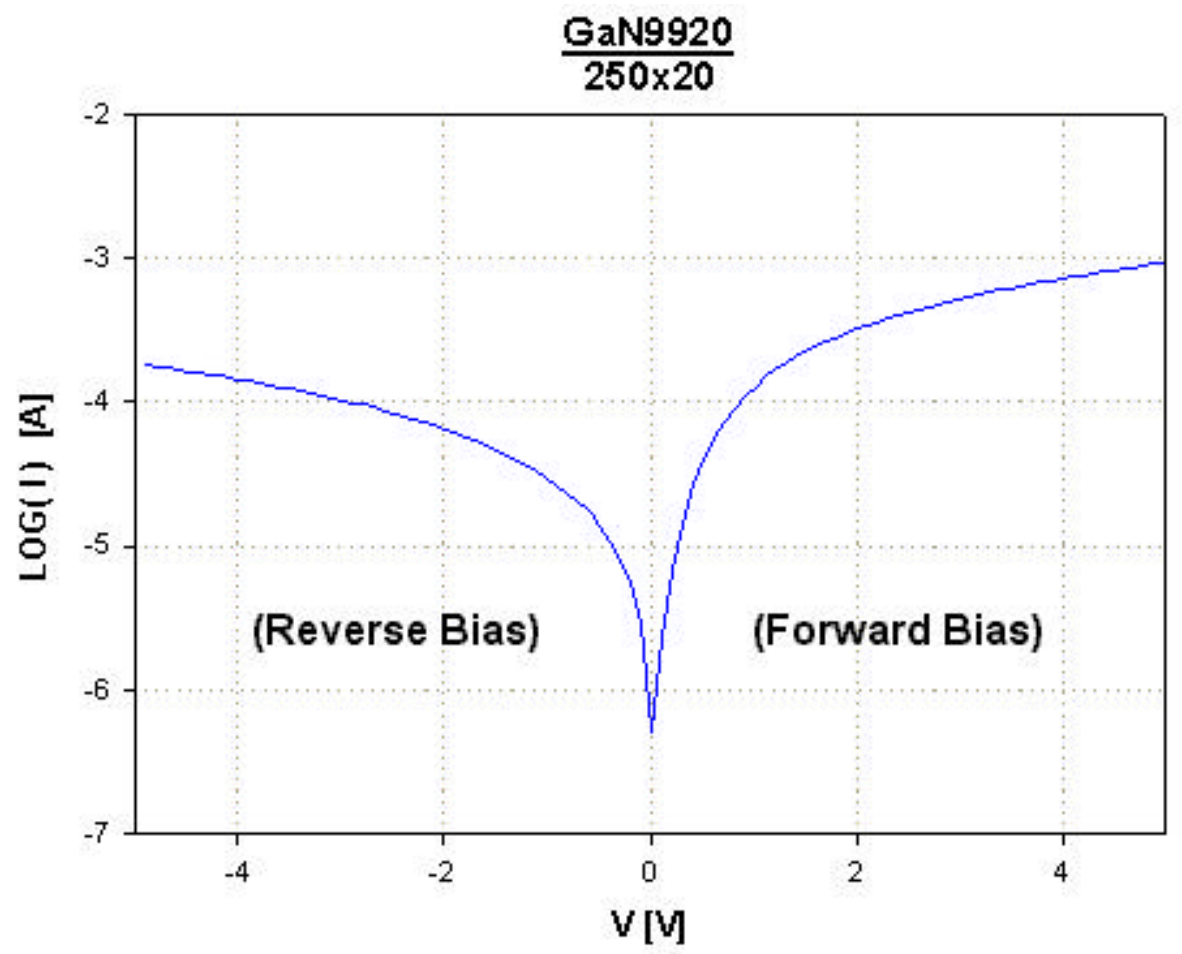

Figure 4.25: $\log \mathrm{I}-\mathrm{V}$ plot of a $250 \mu \mathrm{m}$ dot with $20 \mu \mathrm{m}$ of separation on GaN9920.

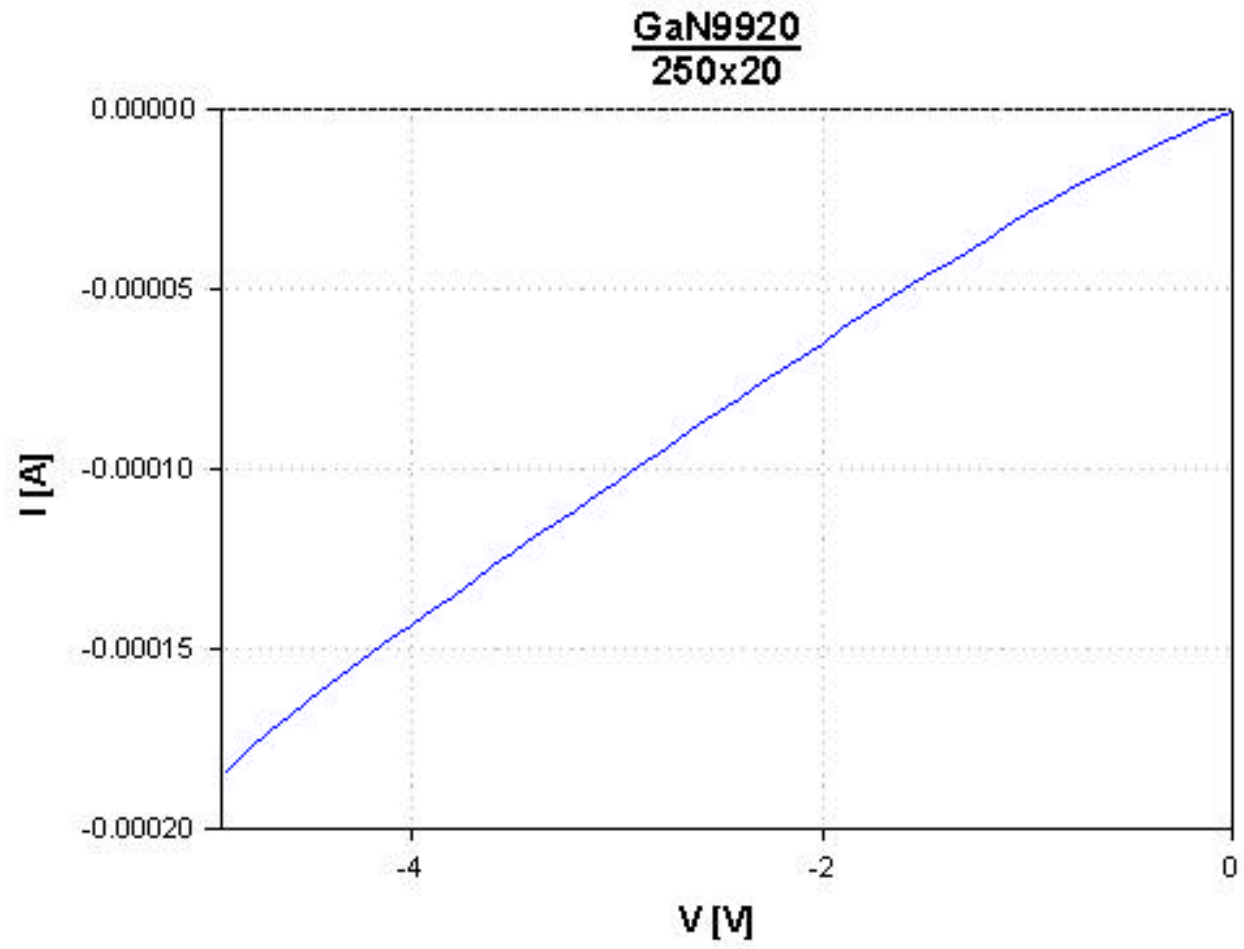

Figure 4.26: Saturation current of a $250 \mu \mathrm{m}$ dot with $20 \mu \mathrm{m}$ of separation on GaN9920. 


\subsubsection{LOSS TANGENT}

The dissipation factor was measured against frequency at zero applied bias voltage. A representative plot, obtained from the same structure $(250 \mu \mathrm{m}$ dot with $20 \mu \mathrm{m}$ of separation between the dot and the ring), is shown on figure 4.27. The point where the loss tangent reaches its minimum is $20 \mathrm{KHz}$. At this frequency the dissipation factor is 0.312 .

\subsubsection{DEPLETION CAPACITANCE AT ZERO BIAS}

The plots of the measured capacitance and conductance as functions of the frequency of a representative contact $(250 \mu \mathrm{m}$ and $20 \mu \mathrm{m}$ of separation) are shown on figures 4.28 and 4.29 . It can be seen for this sample that points between $10 \mathrm{KHz}$ and $40 \mathrm{KHz}$ should produce the most reliable measurement.

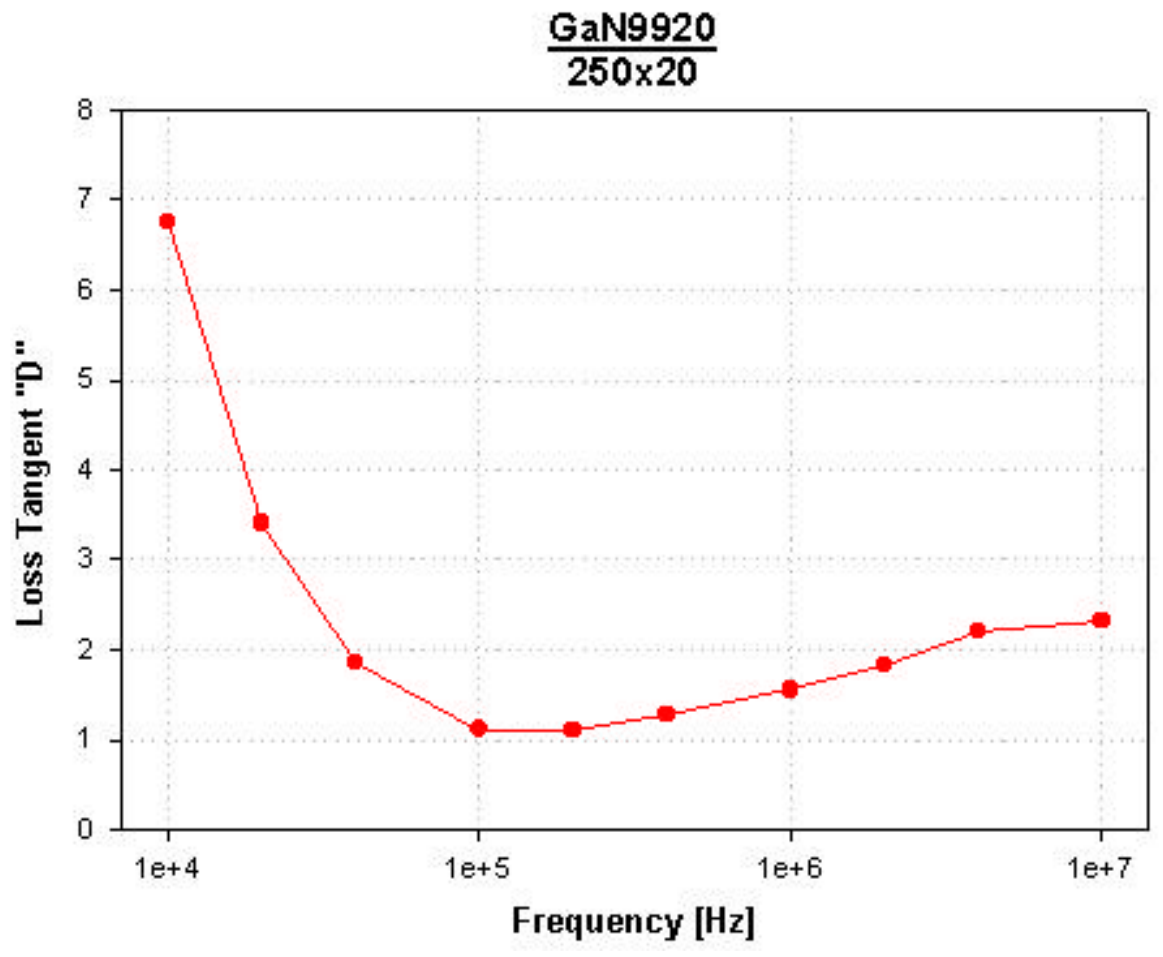

Figure 4.27: Loss tangent versus frequency of a $250 \mu \mathrm{m}$ dot with $20 \mu \mathrm{m}$ of separation on GaN9920. 


\subsubsection{BUILT-IN VOLTAGE}

Two contacts were measured. All contacts had a dot with $250 \mu \mathrm{m}$ of radius, but different separations between their dots and rings. The separations were $20 \mu \mathrm{m}$ and $60 \mu \mathrm{m}$. The best points in frequency determined for the built-in voltage measurement for each of these contacts are shown on table 4.14.

Table 4.14: Points of minimum for the measured contacts on GaN9920.

\begin{tabular}{|c|c|c|c|}
\hline Separation $[\mu \mathrm{m}]$ & Frequency $[\mathbf{H z}]$ & Loss tangent “D”' & Measured $\mathbf{C}_{\mathbf{j o}}[\mathbf{p F}]$ \\
\hline 20 & $200 \mathrm{~K}$ & 1.1 & 44.55 \\
\hline 60 & $100 \mathrm{~K}$ & 1.23 & 32.40 \\
\hline
\end{tabular}

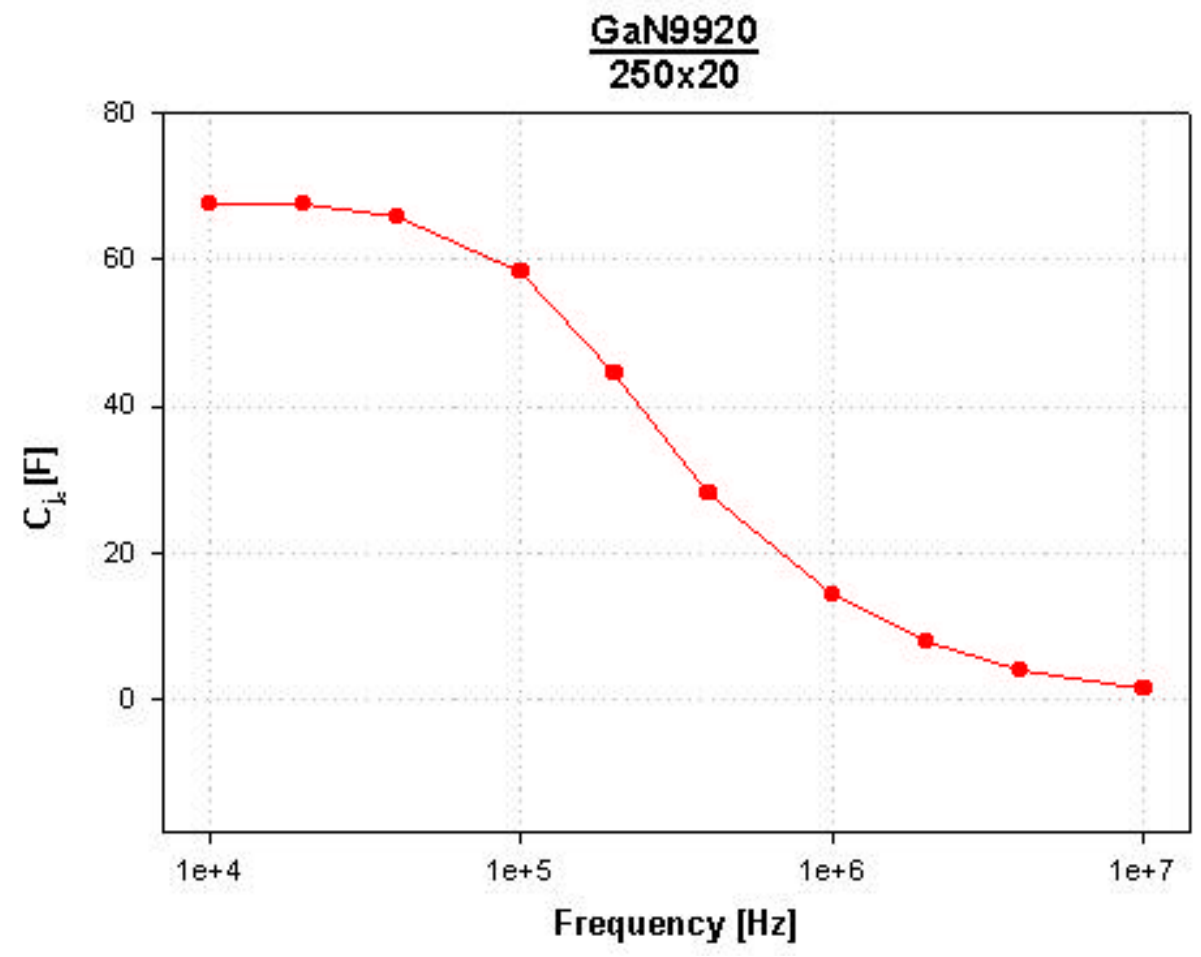

Figure 4.28: Junction capacitance of a $250 \mu \mathrm{m}$ dot with $20 \mu \mathrm{m}$ of separation at zero bias on GaN9920. 


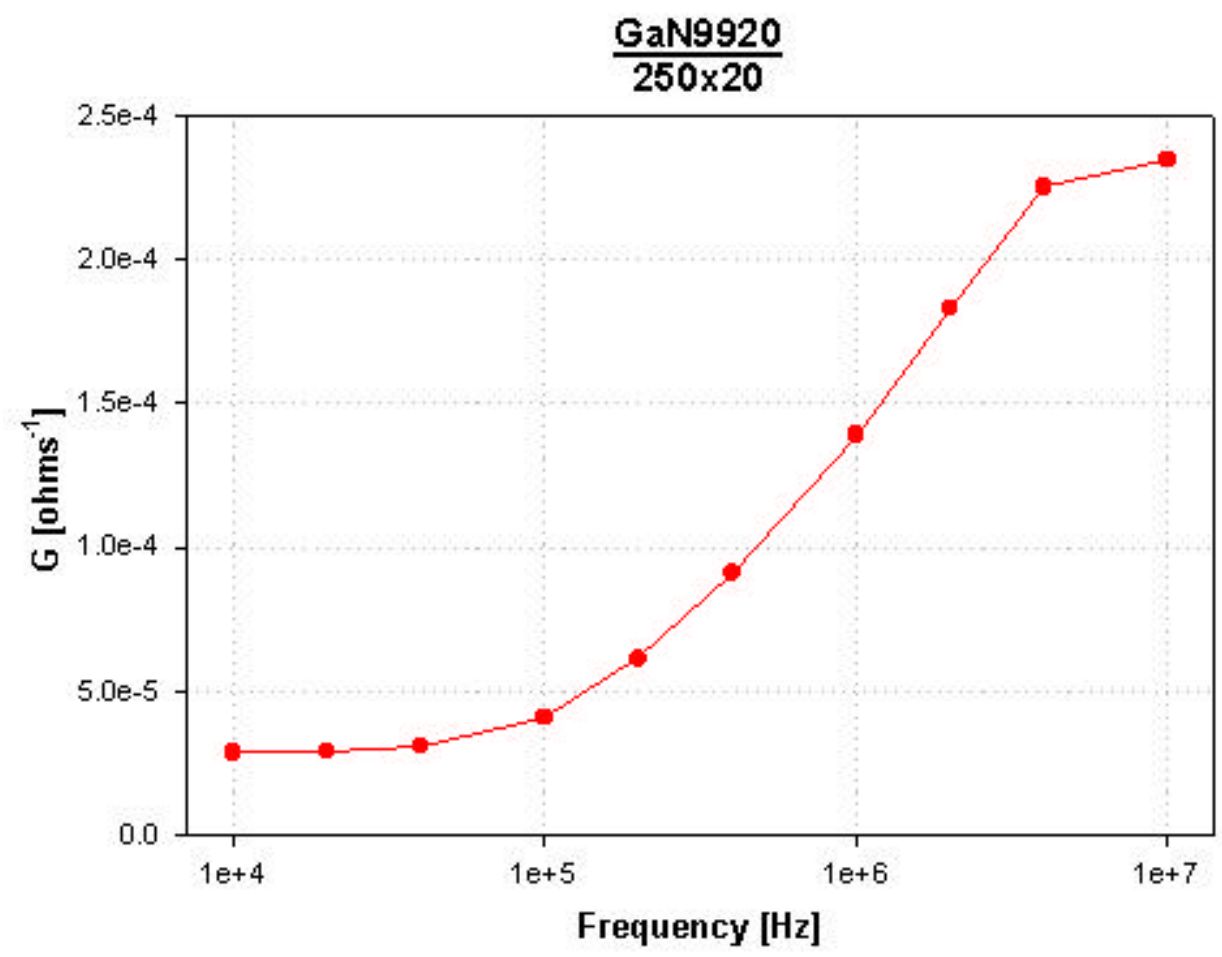

Figure 4.29: Junction conductance of a $250 \mu \mathrm{m}$ dot with $20 \mu \mathrm{m}$ of separation at zero bias on GaN9920.

The calculated mean and standard deviation of the loss tangent were 1.165 and 0.065 respectively. The calculated mean and standard deviation for the measured capacitance at no bias were $38.47 \mathrm{pF}$ and $6.075 \mathrm{pF}$ respectively. Based on the results from table 4.6, C-V curves were taken at those specific frequencies. The $\mathrm{C}-\mathrm{V}$ curve of a representative contact $250 \mu \mathrm{m}$ dot with $20 \mu \mathrm{m}$ of separation) is shown on figure 4.30. The plot of $\mathrm{C}^{-2} v s$. V obtained from the same contact is shown on figure 4.31. The values obtained for the slope and intercept for each of the C ${ }^{2}$ vs. V curves are shown on table 4.15. Using the values of table 4.15, a program (appendix C) applies equation $2.20 \mathrm{~b}$ to calculate the built-in voltage. These results are found on table 4.16 .

Table 4.15: Slope and intercept of $\mathrm{C}^{-2}$ vs. V curves of four contacts on GaN9920.

\begin{tabular}{|c|c|c|}
\hline Separation $[\mu \mathbf{m}]$ & Slope $\mathbf{x} \mathbf{1 0}^{\mathbf{2 0}}\left[\mathbf{F}^{-2} \mathbf{V}^{-1}\right]$ & Intercept $\mathbf{x 1 0}{ }^{\mathbf{2 0}}\left[\mathbf{F}^{-\mathbf{2}}\right]$ \\
\hline 20 & -6.01 & 4.63 \\
\hline 60 & -12.0 & 9.50 \\
\hline
\end{tabular}


Table 4.16: Calculated built-in voltages for the measured contacts on GaN9920.

\begin{tabular}{|c|c|}
\hline Separation $[\boldsymbol{\mu} \mathbf{m}]$ & Built-in Voltage [V] \\
\hline 20 & 0.80 \\
\hline 60 & 0.82 \\
\hline
\end{tabular}

The calculated mean and standard deviation of the built-in voltages were $0.81 \mathrm{~V}$ and $0.01 \mathrm{~V}$ respectively. However, the series resistance is very high for this sample, and the correction factor given by equation $2.23 \mathrm{~b}$ was used to correct the values of the built-in voltage. The new values found are shown in table 4.17.

Table 4.17: Corrected built-in voltages for the measured contacts on GaN9920.

\begin{tabular}{|c|c|}
\hline Separation $[\mu \mathrm{m}]$ & Built-in Voltage $[\mathbf{V}]$ \\
\hline 20 & 1.66 \\
\hline 60 & 1.13 \\
\hline
\end{tabular}

The mean and standard deviation for this set of measurements was $1.395 \mathrm{~V}$ and $\mathrm{XXX}$ respectively. In this case, the values of the built-in voltage are close to what is expected from the theory $(1.55 \mathrm{~V})$.

\subsubsection{CARRIER CONCENTRATION}

A plot of $\mathrm{C}-\mathrm{V}$ at different frequencies is shown on figure 4.32. It is clearly seen on figure 4.32 that there is a shift in the roll-off of capacitance and this is a good indication that interfacetrapped states are present in this sample. The plot only includes the minimum frequency that the equipment can use, and the maximum frequency at which the shift is not noticeable any longer. 


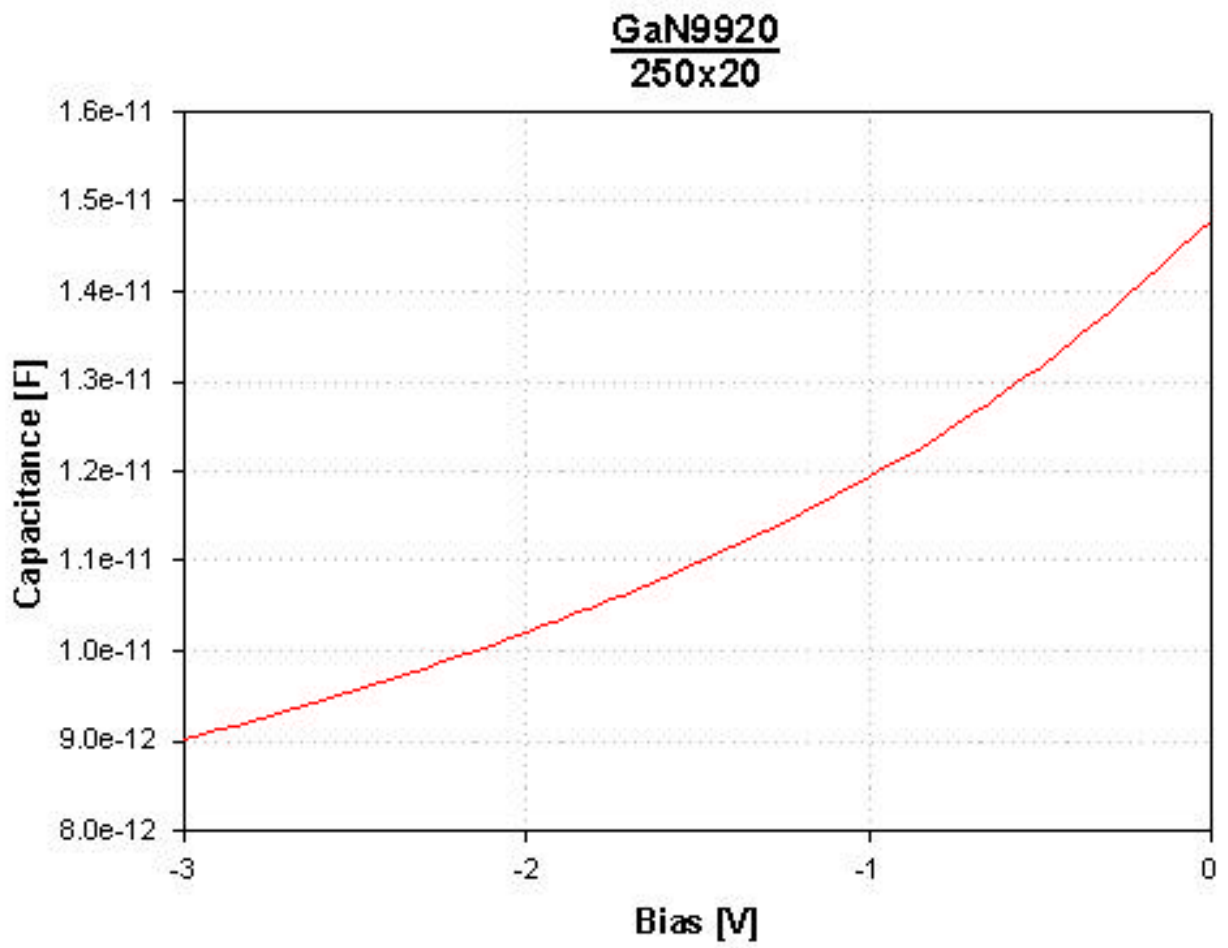

Figure 4.30: C-V of a $250 \mu \mathrm{m}$ dot with $20 \mu \mathrm{m}$ of separation at $400 \mathrm{KHz}$ on GaN9920.

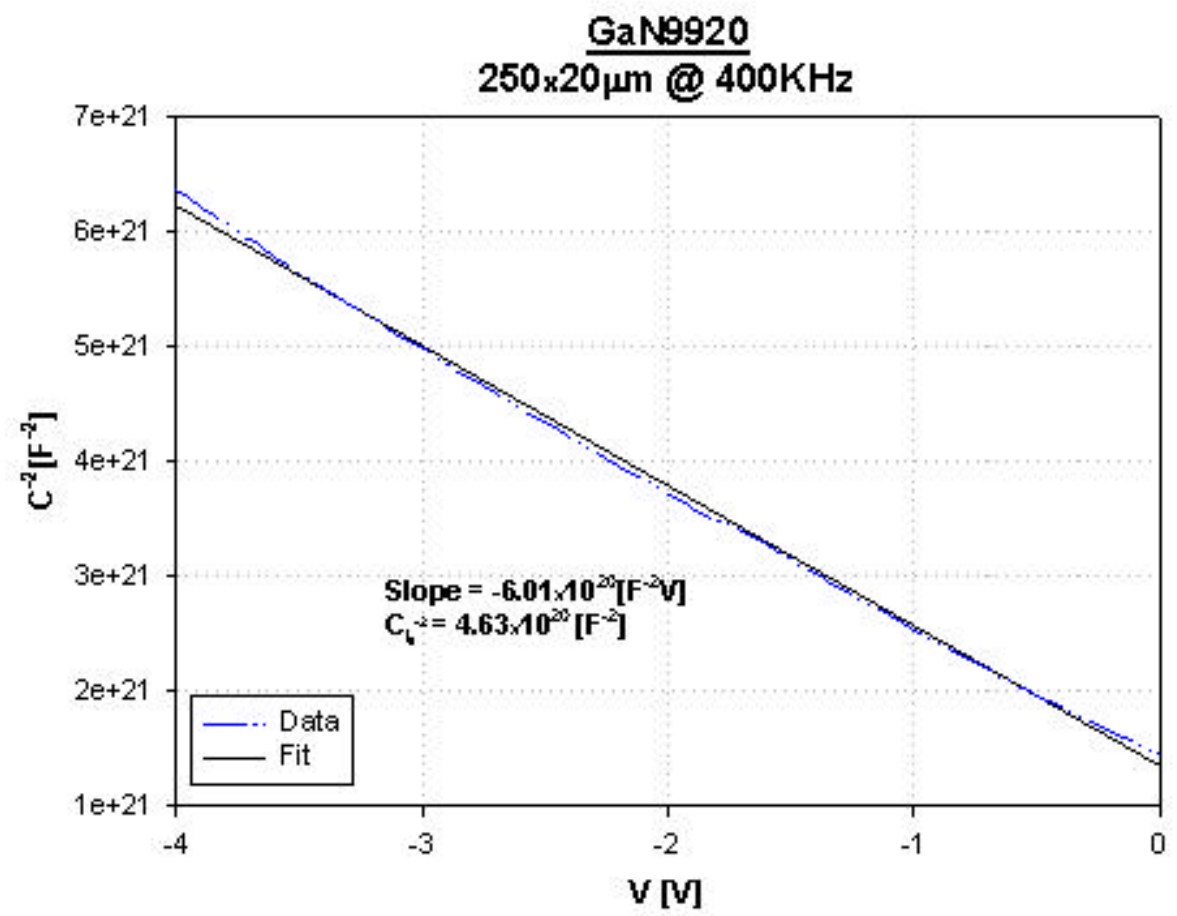

Figure 4.31: $\mathrm{C}^{-2} v s . \mathrm{V}$ of a $250 \mu \mathrm{m}$ dot with $20 \mu \mathrm{m}$ of separation at $400 \mathrm{KHz}$ on GaN9920. 


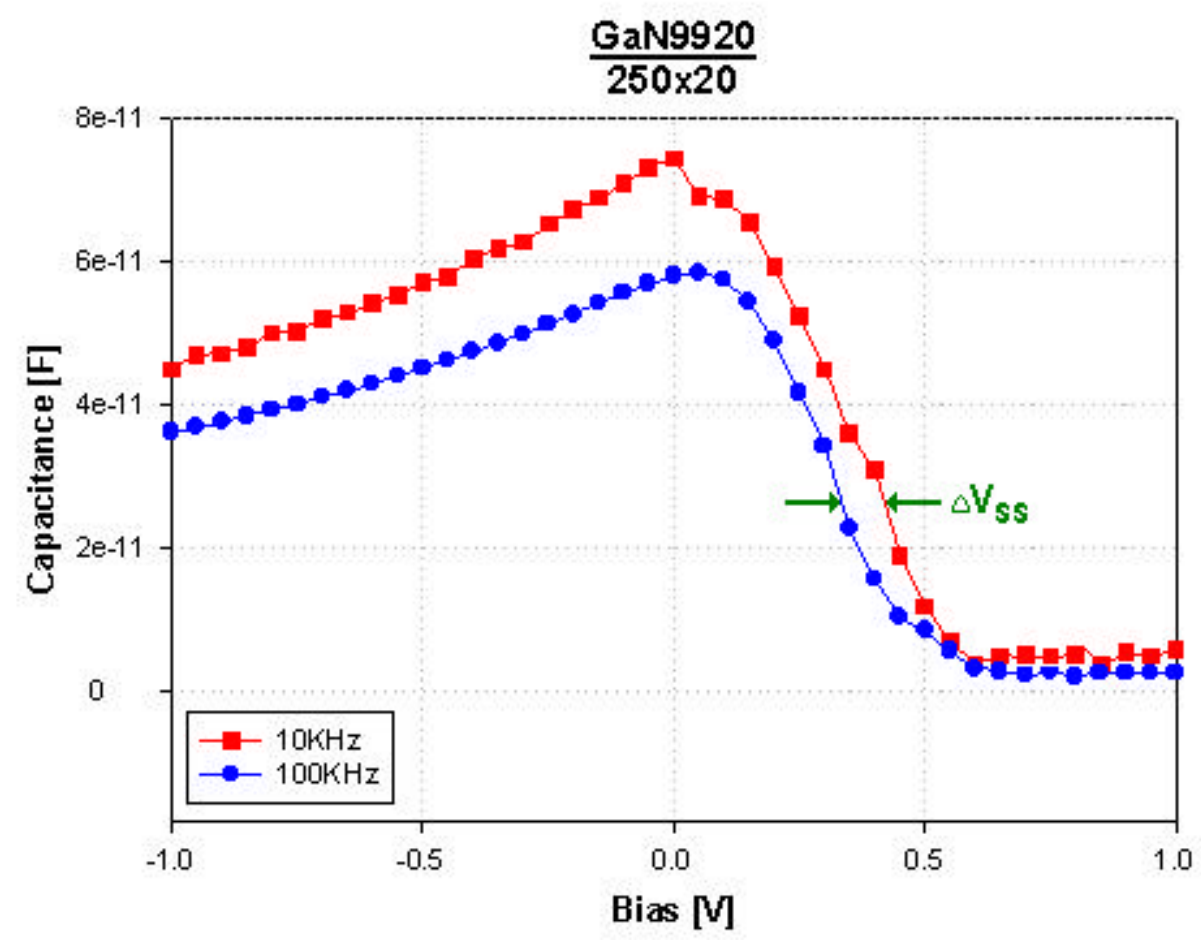

Figure 4.32: C-V curve of a contact on GaN9920 at different test frequencies.

A typical contact $(250 \mu \mathrm{m}$ with $20 \mu \mathrm{m}$ of separation) was measured, and the carrier concentration of the material was extracted at different frequencies. Table 4.18 shows the values found for the carrier concentration. A plot of these values is shown on figure 4.33.

Table 4.18: Carrier concentration of GaN9920 at different frequencies.

\begin{tabular}{|c|c|}
\hline Frequency [Hz] & Carrier Conc. $\mathbf{x 1 0}^{\mathbf{1 5}}\left[\mathbf{c m}^{-3}\right]$ \\
\hline $10 \mathrm{~K}$ & 4.44 \\
\hline $20 \mathrm{~K}$ & 4.17 \\
\hline $40 \mathrm{~K}$ & 4.68 \\
\hline $100 \mathrm{~K}$ & 4.47 \\
\hline $200 \mathrm{~K}$ & 3.79 \\
\hline $400 \mathrm{~K}$ & 2.50 \\
\hline $1 \mathrm{M}$ & 1.10 \\
\hline $2 \mathrm{M}$ & 0.57 \\
\hline $4 \mathrm{M}$ & 0.31 \\
\hline $10 \mathrm{M}$ & 0.16 \\
\hline
\end{tabular}




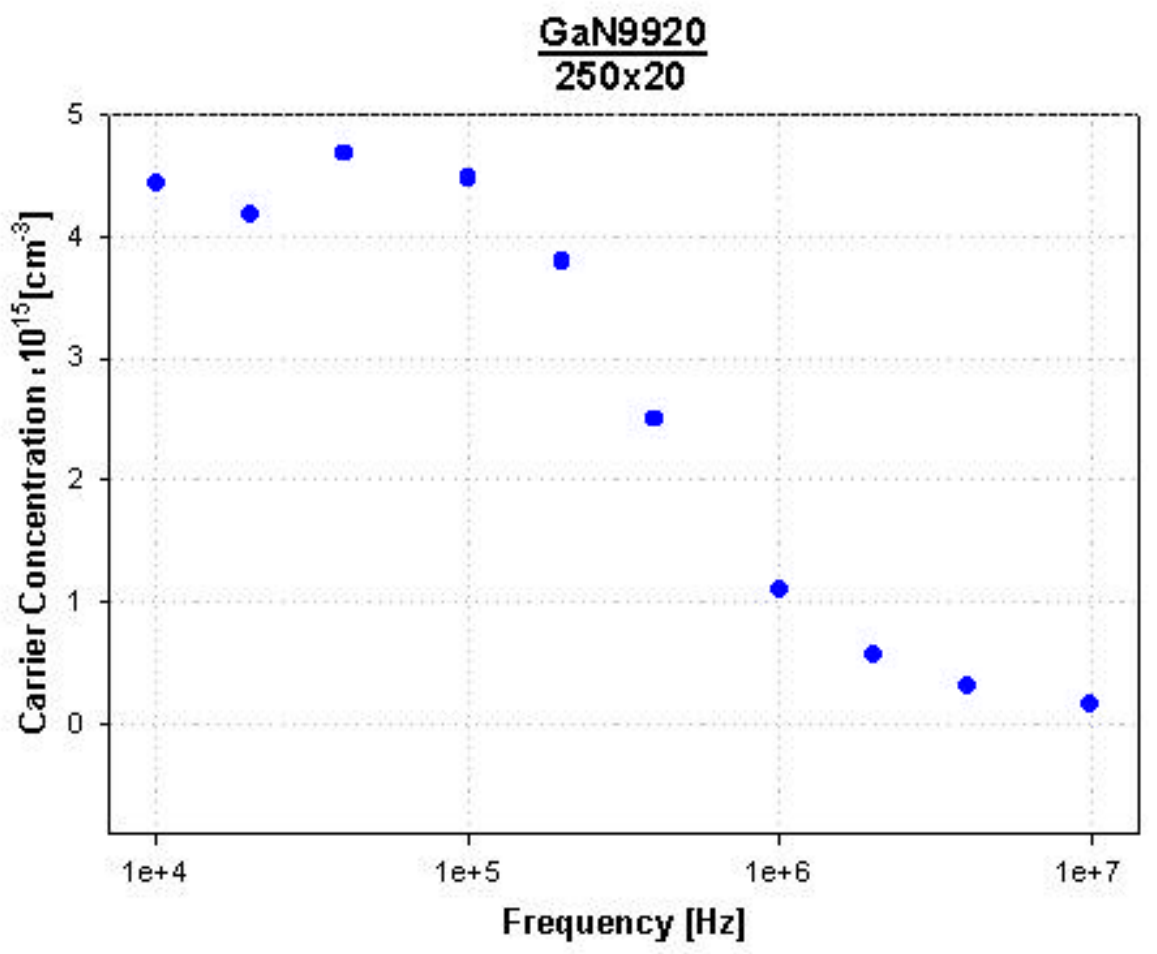

Figure 4.33: Plot of carrier concentration of GaN9920 at different frequencies.

From figure 4.33 one can see that the values of the carrier concentration that are not very dependent on frequency effects are those between $10 \mathrm{KHz}$ and $100 \mathrm{KHz}$. The mean and standard deviation for the values of the carrier concentration between these frequencies are $4.44 \times 10^{15} \mathrm{~cm}^{-3}$ and $0.18 \times 10^{15} \mathrm{~cm}^{-3}$. It was shown on figure 4.27 that the capacitance reading has fewer losses at frequencies near $100 \mathrm{KHz}$. The value of the carrier concentration calculated at this frequency was $4.47 \times 10^{15} \mathrm{~cm}^{-3}$ as shown on table 4.17 .

The product of the series resistance and the parallel conductance was 0.2 for the contact with $20 \mu \mathrm{m}$ of separation and 0.085 for the contact with $60 \mu \mathrm{m}$ of separation. Since these products are high in this sample, a low frequency correction factor (Eq. 2.22c) was used to adjust the values of the carrier concentration. The corrections were made using the values of the series resistance given on table 4.5 and the conductance as shown on figure 4.18. The adjusted values of the carrier concentration that are in the flat region of the curve are shown on table 4.19. 
Table 4.19: Adjusted values of the carrier concentration for GaN9920.

\begin{tabular}{|c|c|}
\hline Frequency $[\mathrm{Hz}]$ & Carrier Conc. $\mathbf{x 1 0}{ }^{\mathbf{1 5}}\left[\mathbf{c m}^{-3}\right]$ \\
\hline $10 \mathrm{~K}$ & 3.21 \\
\hline $20 \mathrm{~K}$ & 3.01 \\
\hline $40 \mathrm{~K}$ & 3.38 \\
\hline $100 \mathrm{~K}$ & 2.82 \\
\hline
\end{tabular}

The mean and the standard deviation of the new values of the carrier concentration were calculated to be $3.11 \times 10^{15} \mathrm{~cm}^{-3}$ and $0.21 \times 10^{15} \mathrm{~cm}^{-3}$ respectively. The carrier concentration found by Hall was $1.25 \times 10^{15} \mathrm{~cm}^{-3}$. In this case the results found by the two measurements were close, though a possible explanation for the difference that could be considered is the same stated for GaN9906E. Another point that can be analyzed is the width of the depletion layer. For this sample, using equation $2.14 \mathrm{~b}$ with the theoretical value of the built-in voltage, and the carrier concentration found by $\mathrm{CV}$, the width of the depletion layer is $0.72 \mu \mathrm{m}$ at zero bias, and $1.49 \mu \mathrm{m}$ at $-5 \mathrm{~V}$. If the same calculation is performed with the value of the carrier concentration obtained by Hall, the width of the depletion width is $1.042 \mu \mathrm{m}$ at zero bias, and $2.14 \mu \mathrm{m}$ at $-5 \mathrm{~V}$. 


\section{Chapter 5 - Discussion, Summary, and Future Work}

\subsection{DISCUSSION}

In this study, all the experimental behaviors seen for both the $\mathrm{Si}$ and $\mathrm{GaN}$ samples with large radius $\mathrm{C}-\mathrm{V}$ test structures are captured by the equivalent circuit models discussed. However, the variation in behavior with dot radius has not been adequately addressed. This behavior can be seen in the Si sample treated in chapter 4 .

Five dot sizes on silicon were measured, all of them with $20 \mu \mathrm{m}$ of separation between their dots and their rings. The results found for the carrier concentration are found on table 5.1. A plot of these values are shown on figure 5.1.

Table 5.1: Measured values of carrier concentration for different dot sizes.

\begin{tabular}{|c|c|c|}
\hline Radius $[\mu \mathbf{m}]$ & Carrier Concentration $\left[\mathbf{c m}^{-3}\right]$ & Capacitance at $\mathbf{- 5} \mathbf{V}[\mathbf{p F}]$ \\
\hline 50 & $12.30 \times 10^{15}$ & 1.61 \\
\hline 100 & $7.30 \times 10^{15}$ & 2.39 \\
\hline 150 & $2.67 \times 10^{15}$ & 3.75 \\
\hline 200 & $1.50 \times 10^{15}$ & 5.5 \\
\hline 250 & $8.44 \times 10^{14}$ & 6.92 \\
\hline
\end{tabular}

Due to the high resistivity of the substrates, and the geometry of contacts used in this study, it is believed that the electric field, and therefore depletion region, are not uniform, yielding a different variation of the depletion layer width with voltage other than the abrupt junction dependence.

A mathematical manipulation can be carried on to analyze such behavior. It was seen on chapter 2 that the carrier concentration of a material is a function of the capacitance as shown in equation $2.19 \mathrm{~b}$. This equation can be manipulated to be more easily visualized. Equation 5.1 shows the result of this manipulation. 


$$
N=\frac{2 \varepsilon_{S}}{A^{2} q \varepsilon_{s} \frac{\partial C^{-2}}{\partial V}}
$$

Combining equation 5.1 with equation $2.17 \mathrm{c}$ one gets one expression in terms of the width of the depletion layer. This last expression is given by equation 5.2.

$$
N=\frac{2 \varepsilon_{S}}{q \cdot \frac{\partial W^{2}}{\partial V}}
$$

This equation indicates that the less the depletion width varies with the applied voltage the larger the carrier concentration should be. One should expect the width of the depletion layer over the dot area to not vary as much as the width near the edge due to the voltage drop and electric field in highly resistive samples. A consequence is that the measured carrier concentration through $\mathrm{C}-\mathrm{V}$ measurements should indicate a value larger than its correct value.

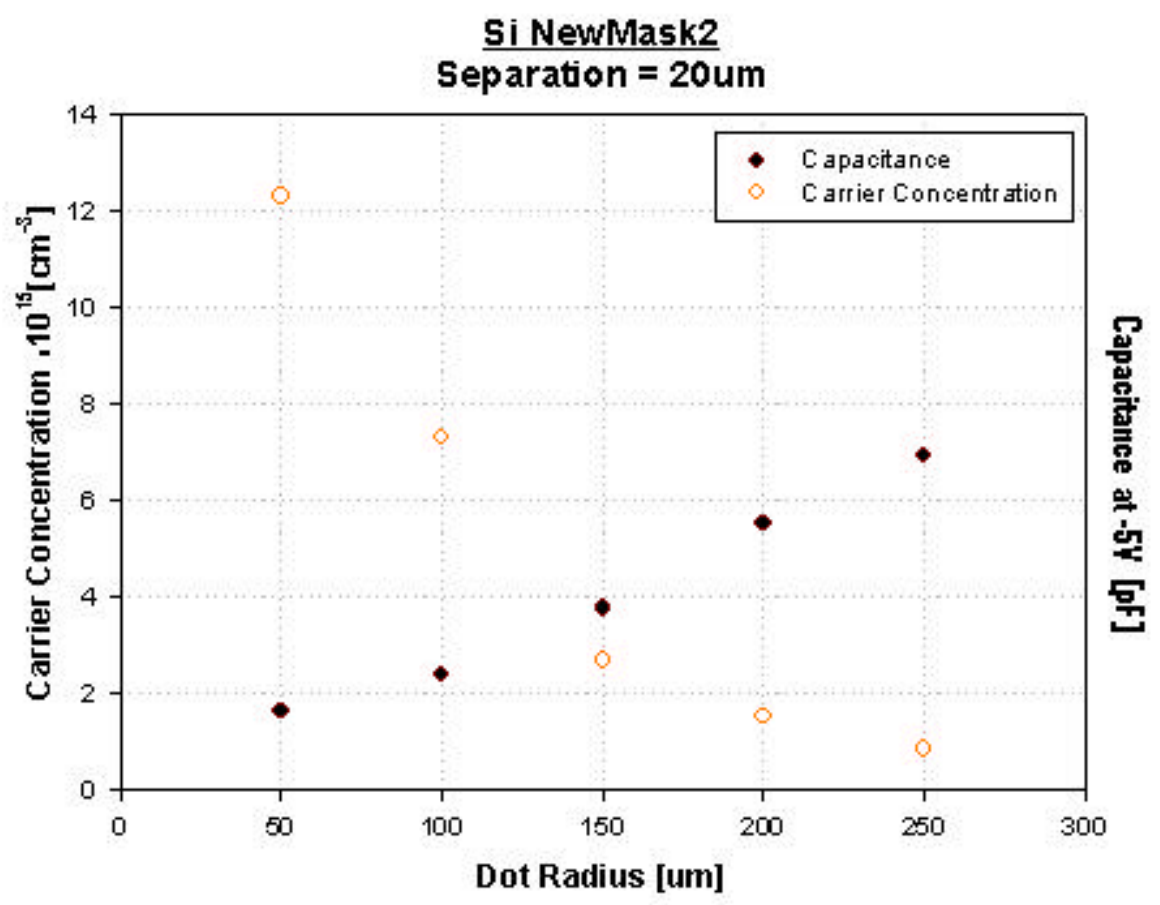

Figure 5.1: Carrier concentration as a function of dot radius. 
A fitting of figure 5.1 has been done in order to obtain an empirical relation for this behavior. The fit suggests that the carrier concentration has an exponential dependence with the dot radius. The equation found that fits figure 5.1 is given by equation 5.3a. A more general form of this equation is given by equation $5.3 \mathrm{~b}$.

$$
\begin{gathered}
N=7.54 \cdot 10^{14} \cdot(1+95 \cdot \exp (-240 \cdot \text { Radius })) \\
N=N_{0} \cdot(1+\alpha \cdot \exp (-\beta \cdot \text { Radius }))
\end{gathered}
$$

The silicon sample is the same studied in section 4.2, its nominal carrier concentration is between $6.3 \times 10^{14} \mathrm{~cm}^{-3}$ and $10^{15} \mathrm{~cm}^{-3}$. In the limit case where the dot radius tends to infinity, the carrier concentration given by equation $5.3 \mathrm{a}$ tends to $7.54 \times 10^{14} \mathrm{~cm}^{-3}$ that is within the nominal range of the carrier concentration. This is a good indication that equation $5.3 \mathrm{~b}$ could be possibly used to fit experimental data and find the carrier concentration in the limit case where the dot radius tends to infinity. Combining equations $5.3 \mathrm{~b}$ and equation 5.2 one finds an expression for the variation of the depletion width with respect to the applied voltage that empirically takes into account the effects of the dot size. This expression is given by equation 5.4.

$$
\frac{\partial W^{2}}{\partial V}=\frac{2 \varepsilon_{S}}{q N_{0} \cdot(1+\alpha \cdot \exp (-\beta \cdot \text { Radius }))}
$$

A plot of equation 5.4 is shown on figure 5.2. This plots suggests that as the dot radius is made smaller, the capacity of the depletion width to change with the applied voltage decreases. Considering this effect and analyzing equation 5.2 one concludes that the reading of carrier concentration should be affected, and the result should be a higher carrier concentration than expected. This effect on the reading of the carrier concentration is what is observed on figure 5.1.

While the results of this analysis are only preliminary, they suggest that when $\mathrm{C}-\mathrm{V}$ measurements are made using coplanar structures on highly resistive substrates, one should pay attention to the dot size and use large dot areas. 


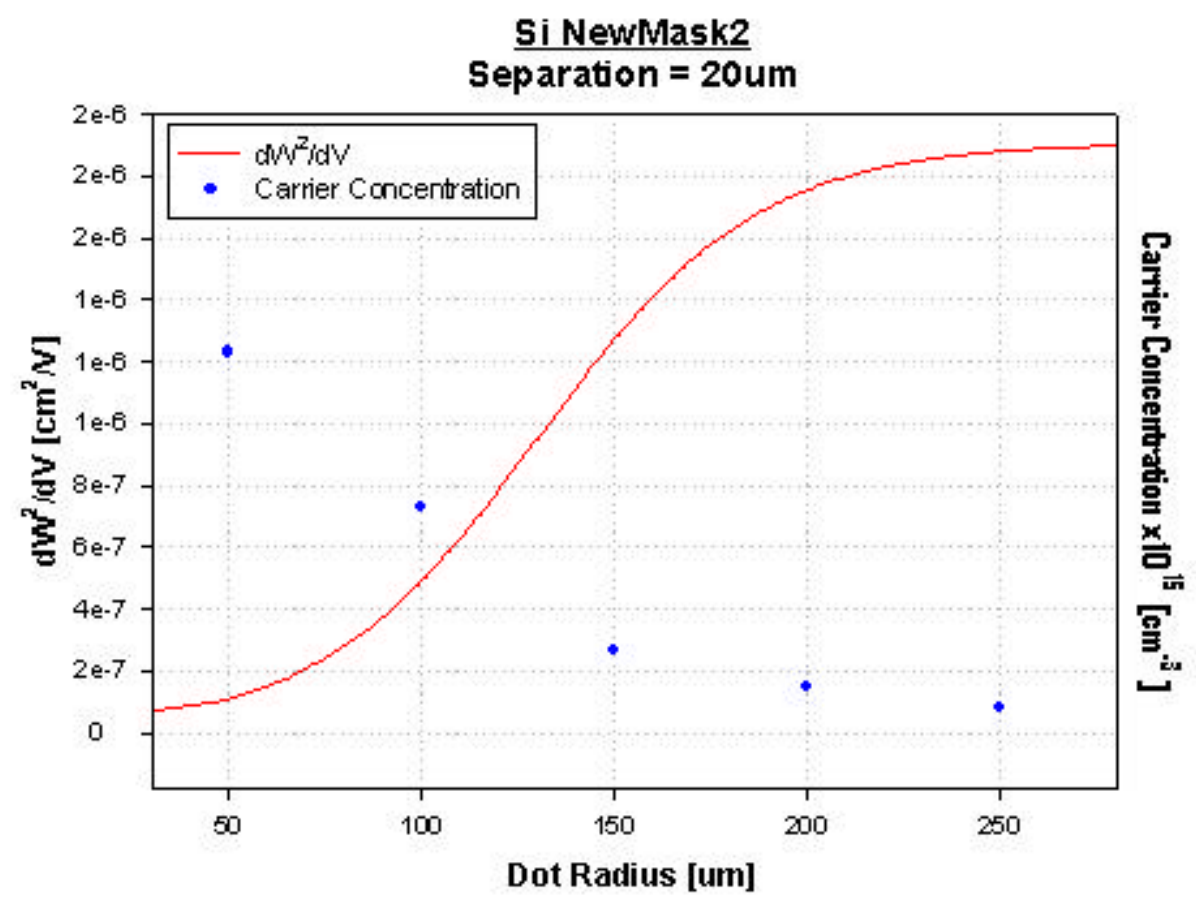

Figure 5.2: Variation of the depletion width with respect to the applied voltage as a function of the dot radius.

\subsection{SUMMARY}

In summary this thesis work has established a experimental and theoretical foundation for the measurement of capacitance, built-in voltage, and carrier concentration using capaticancevoltage measurements. The focus of the basic measurements studies has been on high resistivity p-type silicon samples in order to characterize low doping density unintentionally doped n-type GaN. Experimental results have been reported on each sample type. Circuit models were developed to reflect device, nonidealities, criterion, and methodology outlined for accurate measurements.

The first nonideality analyzed was the presence of a series resistance on contacts. This resistance strongly affects the frequency performance of measurements. The first way analyzed to get around this problem was using low frequencies, and making the product rG (series resistance times parallel conductance) much smaller than the unity. The second imperfection seen was the presence of surface states. This analysis establishes a tradeoff in capacitance 
measurements, since when there are interface-trapped charges in the material, the technique requires that the measurement is performed at sufficient high frequencies such that this charges do not influence the measurement.

A good way of assessing how accurate the measurement is, is by analyzing the loss tangent. The loss tangent says how big the parallel conductance is comparing with the capacitive parallel reactance. And in doing so, it shows how trustworthy the measurement is. A good measurement should be performed then, where the dissipation factor is the lowest achievable.

When performing measurements of the capacitance at zero bias or positive bias, one should also be concerned about the effects of the diffusion capacitance that appears in parallel with the depletion capacitance. This additional capacitance also has a dependence with frequency and has its maximum value at very low frequencies. Again, a tradeoff appears and the measurement should be taken at an intermediate frequency and at a bias where the influence of this capacitance is not very strong.

Chapter four showed how the equations presented on chapter two present themselves with the experiments. A silicon sample was used to emulate the behavior of a highly resistive material such as unintentionally doped gallium nitride.

A well-behaved sample of gallium nitride (GaN9906E) was also measured, and its results appear on chapter four. Beyond the problems presented for highly resistive materials in general, gallium nitride showed to have its peculiar problems. The first problem found analyzing this material was its high saturation current. This elevated value of the saturation current suggests that the diffusion capacitance in the sample can be high and interfere in the measurements.

\subsection{FUTURE WORK}

One peculiarity found on this sample of gallium nitride was the shift of the roll-off curves of the capacitance versus voltage at different frequencies. As explained on Chapter 2, this is a good indication of the presence of interface-trapped charges in the material. The same effect was 
found on the sample of silicon. However, these states are very well studied in this material. In the case of gallium nitride, the presence of these states in low carrier concentration samples needs to be further studied.

The performance of contacts on gallium nitride were shown to be somewhat poor compared to those on silicon. If better contacts can be achieved, the tradeoff found in measurements can be reduced and more accurate measurements can be performed. The series resistance, for example, can be reduced as other techniques are used to create Ohmic contacts [13-19]. The reasons why the saturation current is so high in gallium nitride also deserve some attention, and should be decreased in order to make better measurements that depend on the depletion capacitance.

The last point to be discussed is the dependence of the carrier concentration on the dot size. A preliminary discussion of the results found on silicon were shown in the beginning of this chapter. The analysis, however, has its foundations based on empirical ideas. Therefore, this is also a topic that has to be further studied and a more fundamental understanding should be achieved. One way of achieving this is by numerical simulations, that can be performed with CAD tools such as TSUPREM.

$\mathrm{C}-\mathrm{V}$ measurements, properly applied, are an effective measurement technique to assess material parameters as well as nonidealities. This work has sought to establish a firm, basic experimental, and modeling foundation upon which subsequent studies can be built that will enable them to effectively and fully use $\mathrm{C}-\mathrm{V}$ as an experimental tool. 


\section{Bibliography}

[1] S. Nakamura, G. Fasol; The Blue Laser Diode (GaN Based Light Emitters and Lasers); Springer, 1997.

[2] J. A. Majewski, M. Städele, and P.Vogel, MRS Internet Journal of Nitride Semiconductor Research, 1, Article No.1, (1996).

[3] J. H. Edgar, Properties of Group III Nitrides Electronic Materials Information Service (EMIS), London, (1994).

[4] B. J. Baliga and S. Krishna, "Optimization of Recombination Level and Their Capture Cross Section in Power Rectifiers and Thyristors", Solid State Electron., 20, 225 (1977).

[5] W. Schockley, "The Theory of $p$-n Junctions in Semiconductors and p-n- Junction Transistors", Bell Syst. Tech. J., 28, 435 (1949).

[6] Sze, S. M., "Physics of Semiconductor Devices", Wiley-Interscience, (1981).

[7] P. R. Gray and R. G. Meyer, "Analysis and Design of Analog Integrated Circuits", $3^{\text {rd }}$ ed., Wiley (1942).

[8] A. M. Goodman, "Metal-Semiconductor Barrier Height Measurement by the Differential Capacitance Method - One Carrier System”, J. Appl. Phys. 34, 329 (1963).

[9] W. Shockley, "On the Surface States Associated with a Periodic Potential", Phys. Rev., 56, 317 (1939).

[10] D. V. Lang, "Deep-Level Transient Spectroscopy: A New Method to Characterize Traps in Semiconductors", J. Appl. Phys. 45, 3023 (1974).

[11] V. Y. Niskov, and G. A. Kubetskii, "Resistance of Ohmic Contacts Between Metal and Semiconductor Films", Sov. Phys. Semicond. 4, 1553 (1971).

[12] G. S. Marlow, and M. B. Das, "The Effects of Contact Size and Non-Zero Metal Resistance on the Determination of Specific Contact Resistance", Solid State Elec. 25, 92 (1982).

[13] Y. Kribes, and C. T. Foxon, et. al., "Investigation of Aluminum Ohmic Contacts to n-type GaN Grown by Moleculat Beam Epitaxy", Semicond. Sci. Technol. 12, 1500 (1997).

[14] D. B. Ingerly, Y. A. Chang, et. al., "Low Resistance Ohmic Contacts to n GaN and n-AlGaN using NiAl”, Appl. Phys. Let. 77, 3, (2000). 
[15] Q. Z. Liu, T. F. Kuech, et. al., "Room Temperature Epitaxy of Pd Films on GaN under Conventional Vacuum Conditions”, Appl. Phys. Let. 69, 12, (1996)

[16] J. D. Guo, C. Y. Chang, et. al. "Study of Schottky Barriers on n-type GaN grown by Low-Pressure Metalorganic Chemical Vapor Deposition", Appl. Phys. Let. 67, 18, (1995).

[17] Lei Wang, Q. Chen, et. al., "High Barrier Height GaN Schottky Diodes: Pt/GaN and Pd/GaN”, Appl. Phys. Let. 68, 9, (1996).

[18] L. F. Lester, S. D. Hersee, et. al., "Nonalloyed Ti/Al Ohmic Contacts to n-type GaN using High-Temperature Premetallization Anneal”, Appl. Phys. Let. 69, 18, (1996).

[19] J. M. DeLucca, and S. E. Mohney, "Pt Schottky Contacts to n-GaN Formed by Electrodeposition and Physical Vapor Deposition”, Journal of Appl. Phys. 88, 5, (2000).

[20] Summa-Clean SC-15M, Mallinckrodt Trasistar ${ }^{\circledR}$ 8006, Semiconductor Surface Cleaner

[21] Wax Quick Stick ${ }^{\mathrm{TM}}$ 135, South Bay Technology Inc. P/N MWH 135

[22] Cross Reardon, "Ultraviolet Trasmittance in Eyeglass Materials", Journal of Undergraduate Research, University of Florida. Nov. 2000. http://web.clas.ufl.edu/CLAS/jur/0011/reardonfig1.html.

[23] M. Spak, D. Mammato, S. Jain, and D. Durham, "Mechanism and Lithographic Evaluation of Image Reversal in $\mathrm{AZ}^{\circledR} 5214$ Photoresist", Seventh International Technical Conference on Photopolymers. September, 2000. Ellenville, NY.

[24] E. H. Nicollian and A. Goetzberger, "MOS Conductance Technique for Measuring Surface State Parameters", Appl. Phys. Lett. 7 (8), Oct. 1965. Pg. 216-219.

[25] L. J. Van der Pauw, "A Method of Measuring Specifc Resistivity and Hall Effect of Discs of Arbitrary Shape", Phi. Res. Rep. 13(1), Feb. 1958.

[26] D. W. Koon, C. J. Knickerbocker, "What do You Measure When You Measure The Hall Effect?” Rev. Sci. Instrum. 64 (2), Feb. 1993. Pg. 510-513.

[27] D. W. Koon, Arshad A. Bahl, and Edward O. Duncan, "Measurement of Contact Placement Errors in the Van der Pauw Technique", Rev. Sci. Instrum. 60(2), Feb. 1989. Pg. 275-276.

[28] D. W. Koon, "Effect of Contact Size and Placement, and of Resistive Inhomogeneities on Van der Pauw Measurements", Rev. Sci. Instrum. 60(2), Feb. 1989. Pg. 271-274 


\section{Appendix A - IV.VI}

Front Panel

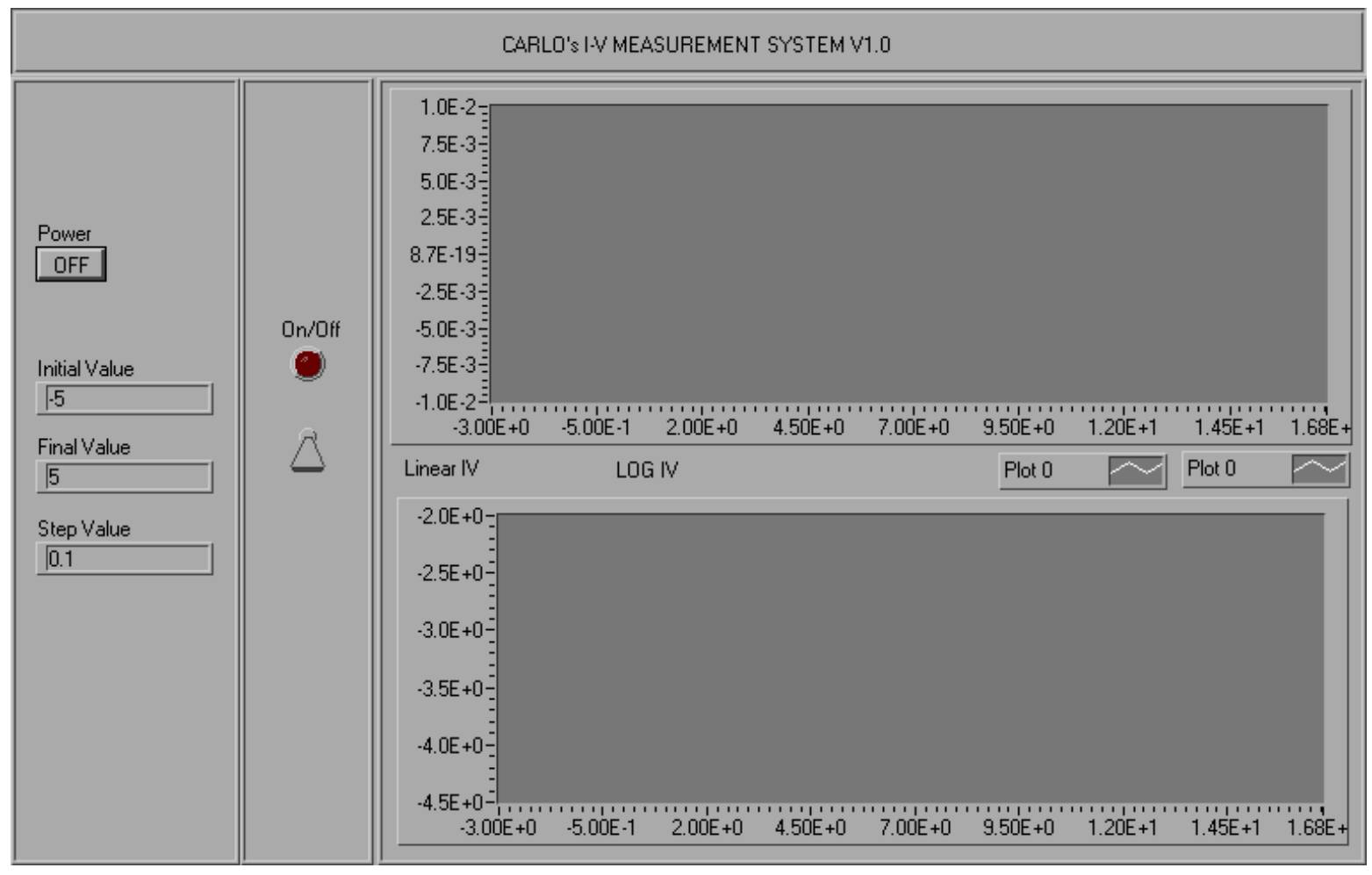




\section{Block Diagram}
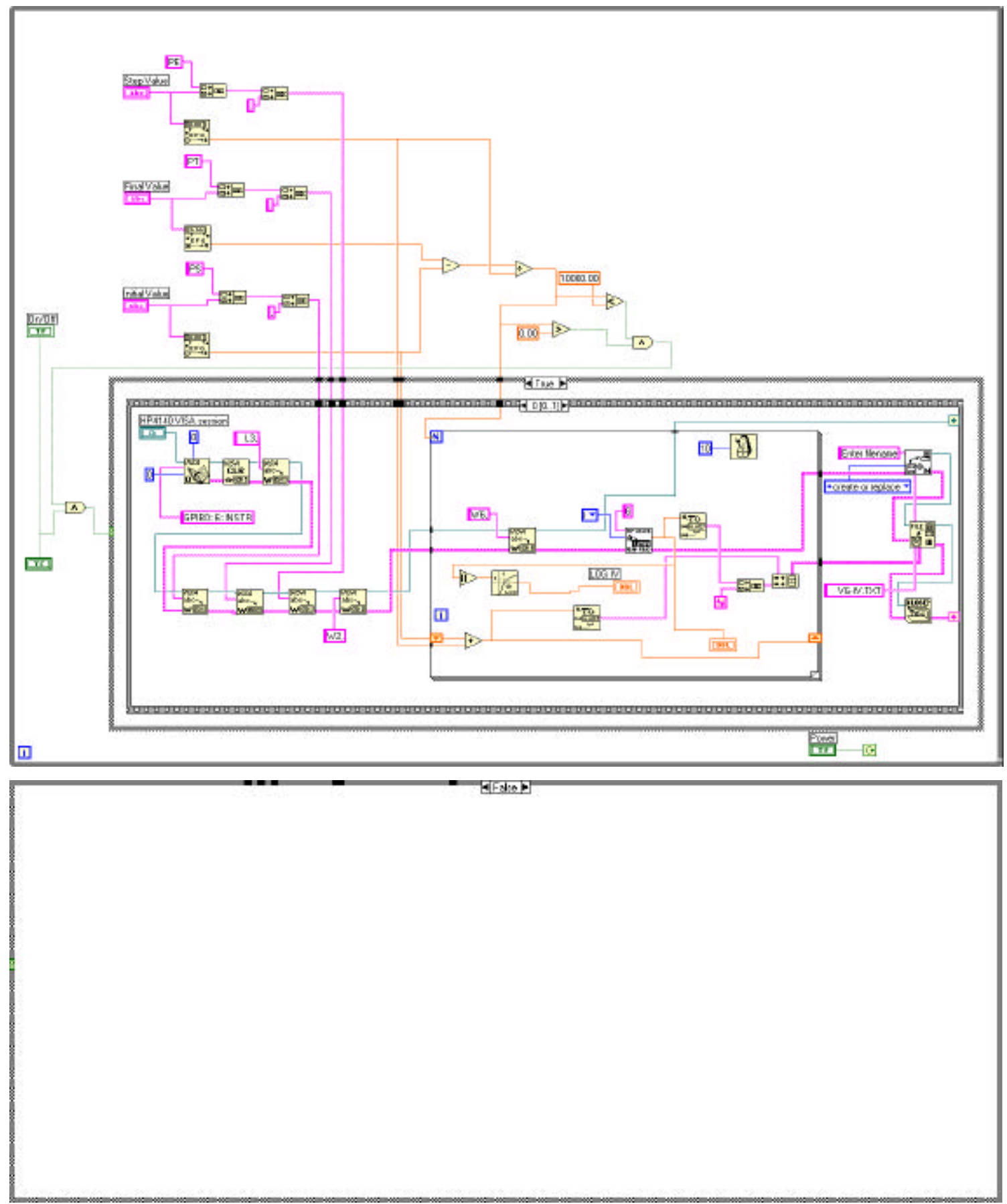
111

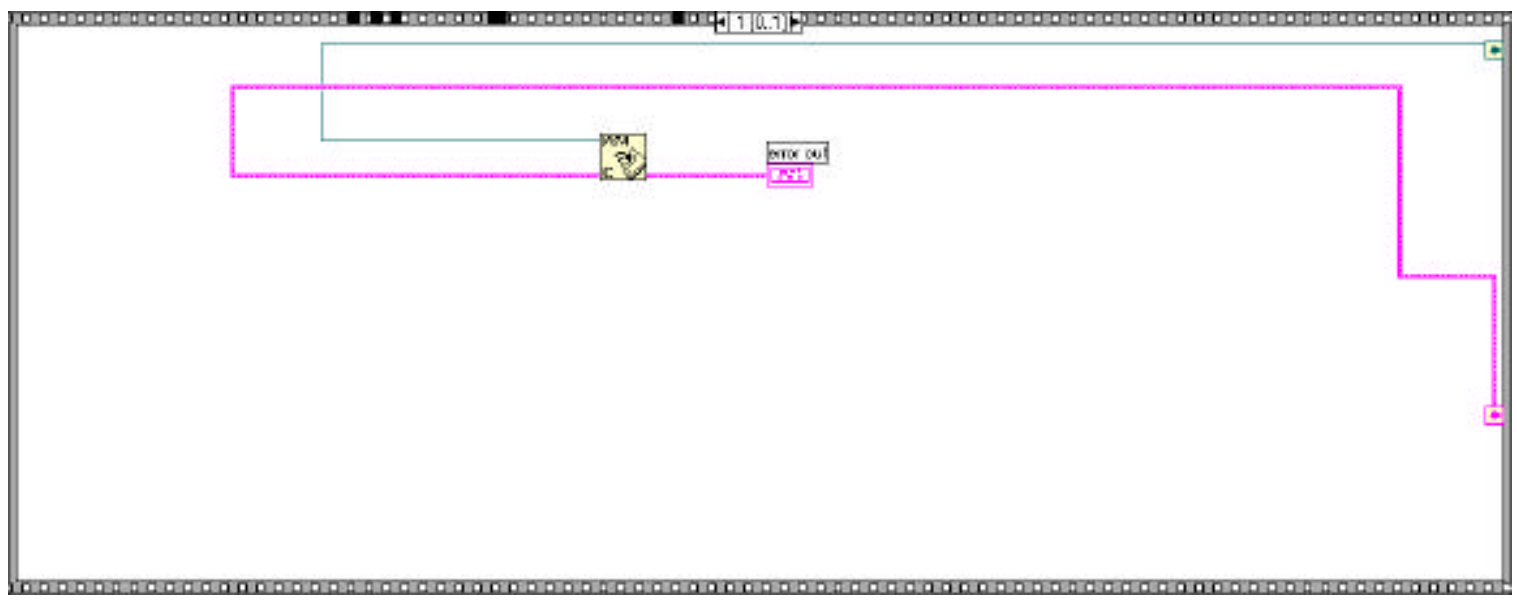




\section{Appendix B - CV.VI}

\section{Front Panel}

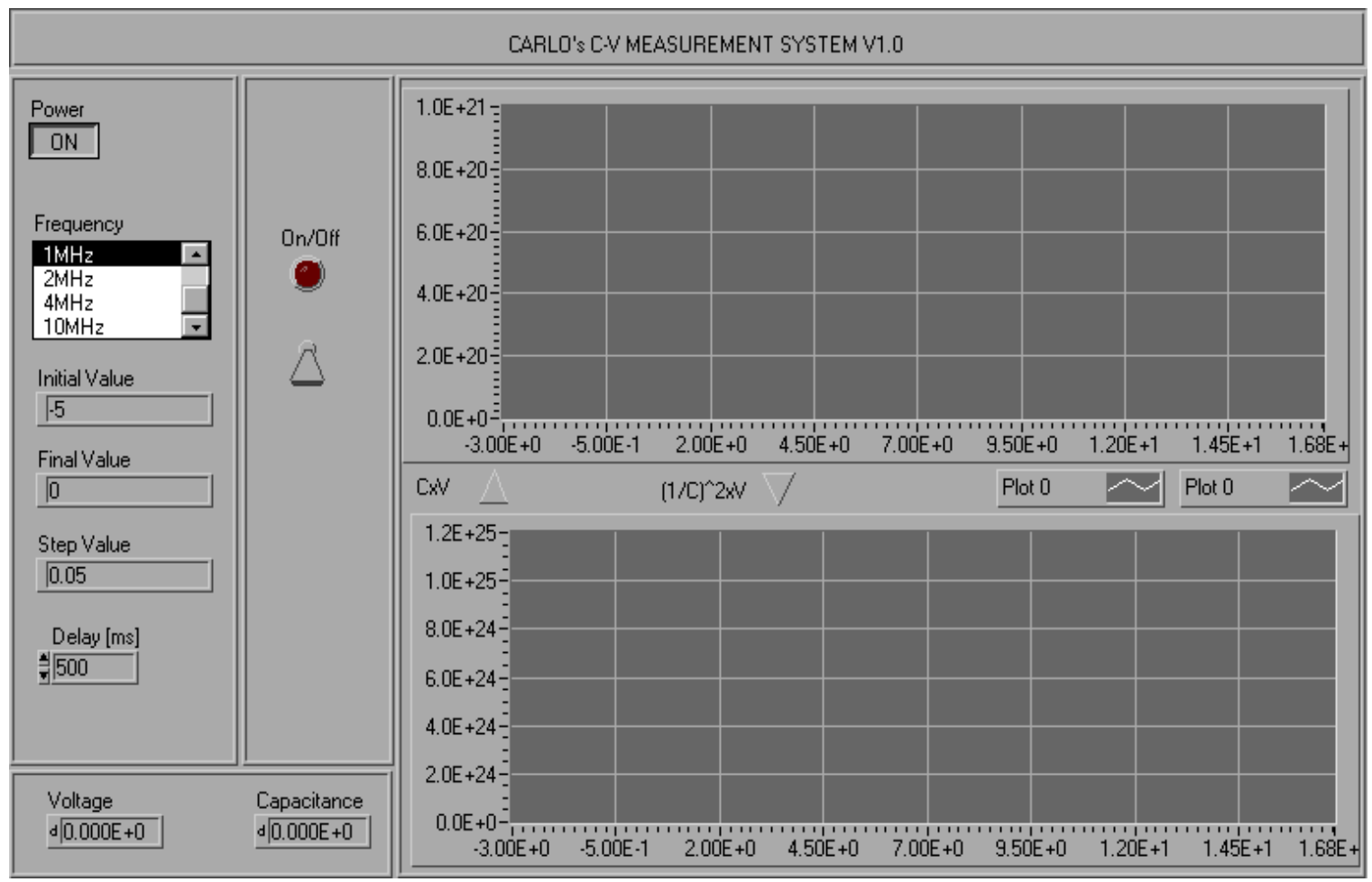




\section{Block Diagram}
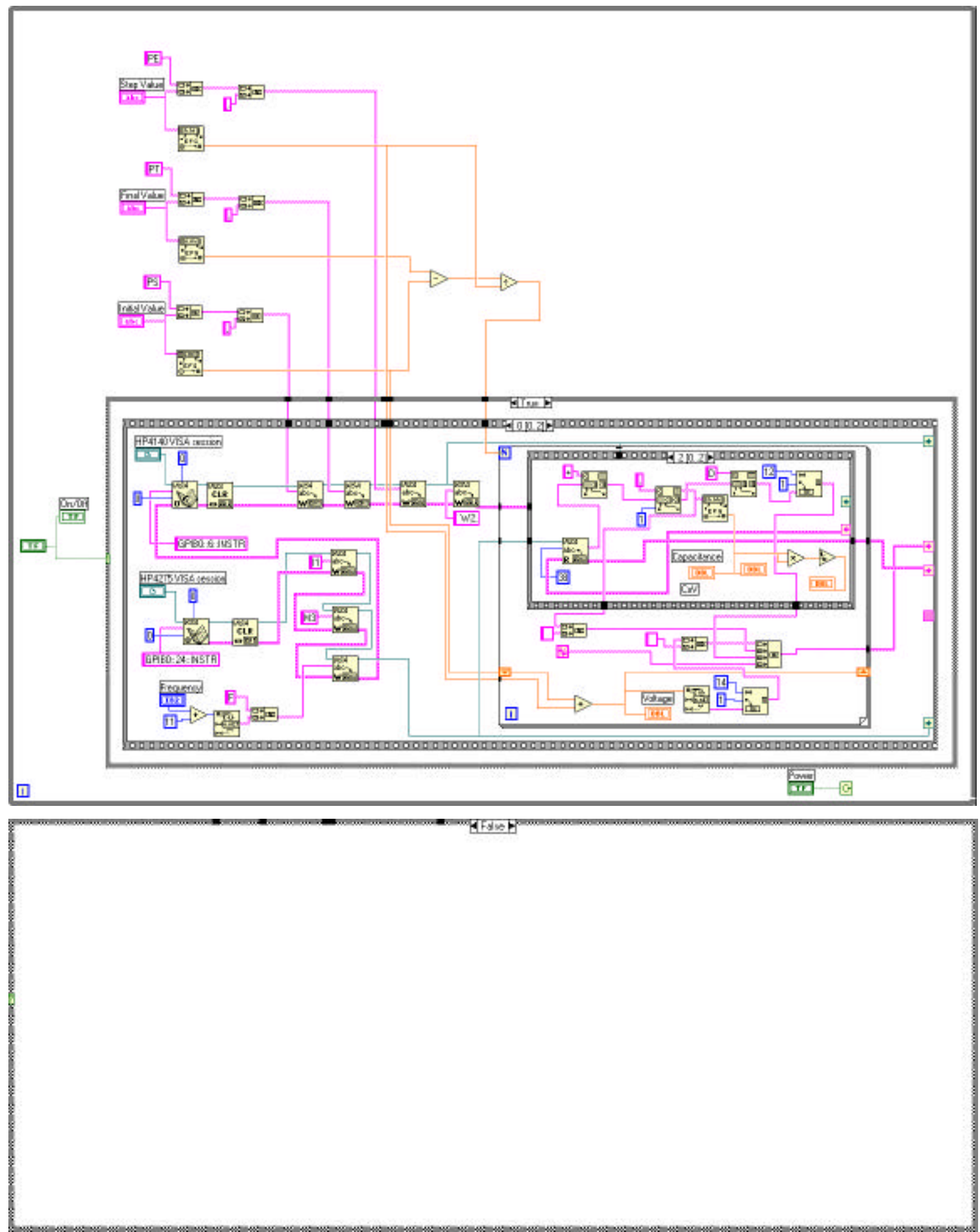

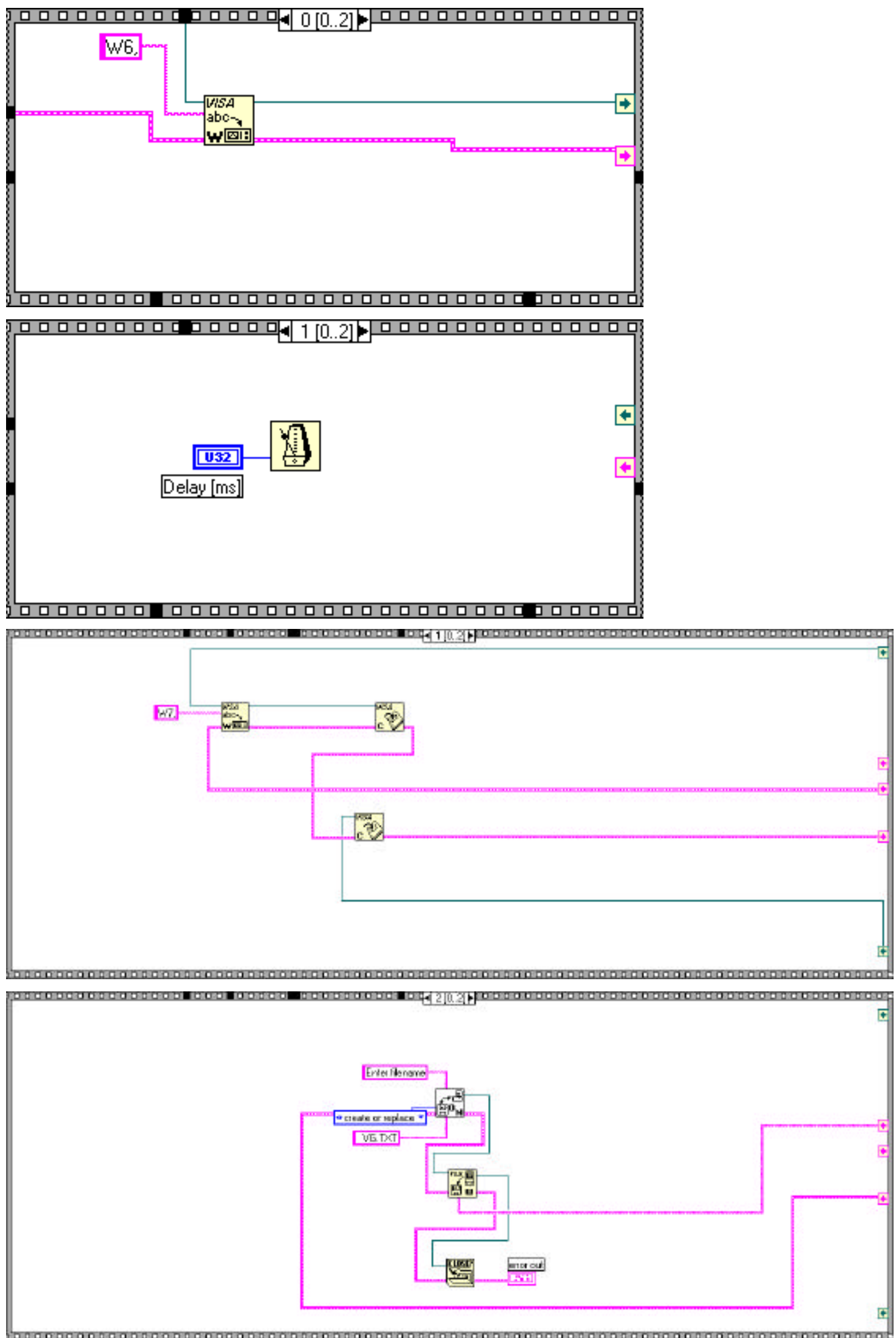


\section{Appendix C-CV.C}

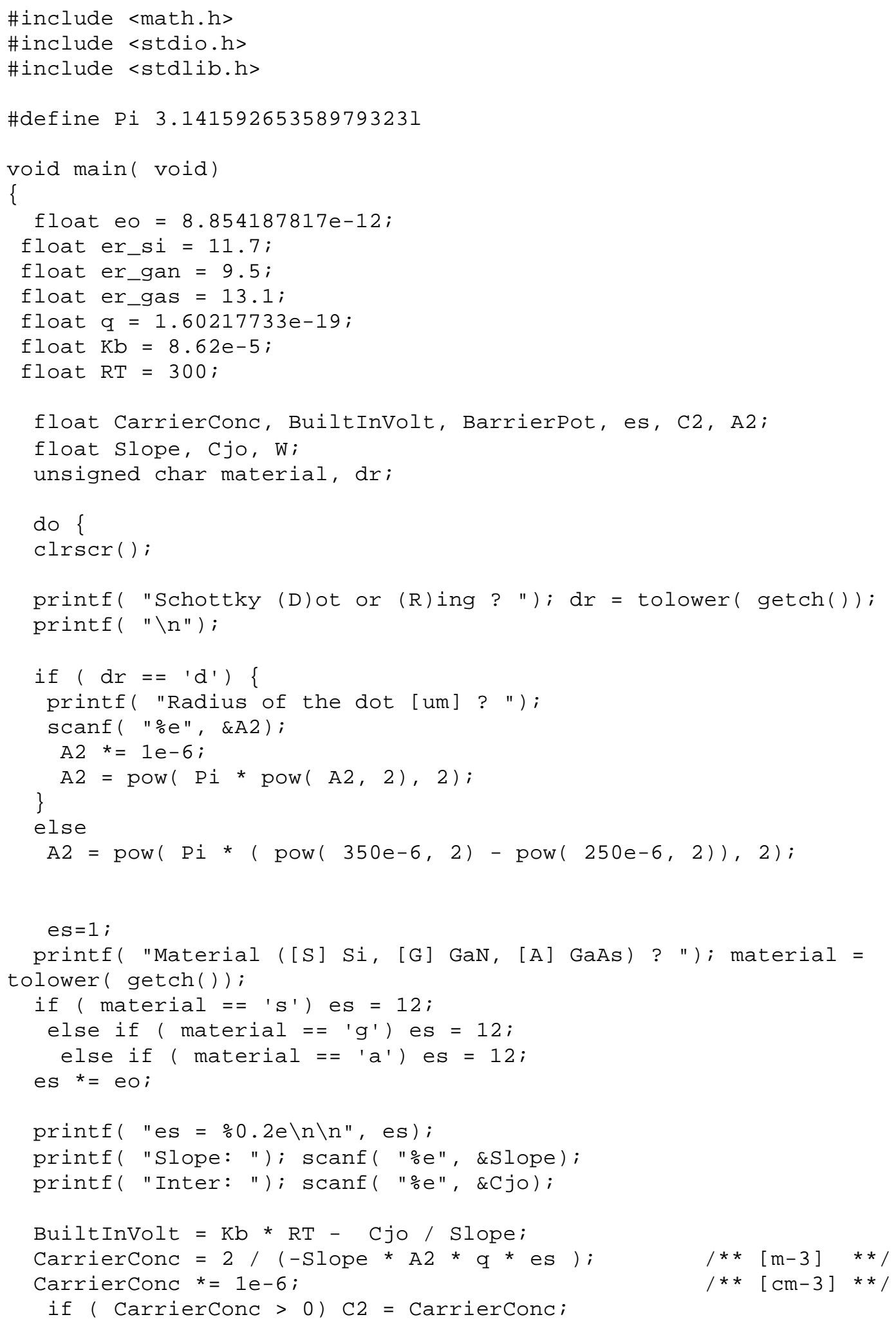




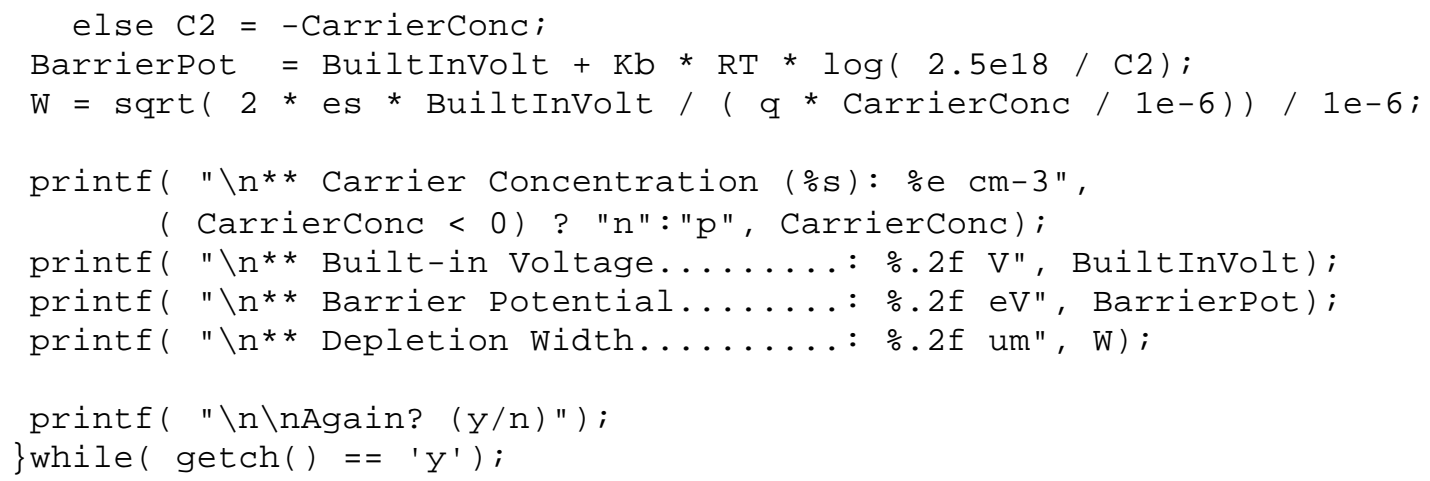




\section{Appendix D - GaAs Results}

Ohmic metal: Au

Schottky metal: Au/Sb/Au

Processing: Same as described for Si on section 3.2. Metals were evaporated instead of sputtered. Mask design 1 (section 3.1) was used.

Hall result: $1.77 \times 10^{18} \mathrm{~cm}^{-3}$ (Appendix F)

Nominal Carrier Concentration: between $1.5 \times 10^{18} \mathrm{~cm}^{-3}$ and $2.7 \times 10^{18} \mathrm{~cm}^{-3}$

Table 6.1: Measurement between a dot and a ring on GaAs.

\begin{tabular}{|c|c|c|c|c|}
\hline \multicolumn{5}{|c|}{ Measurement between a dot and a ring } \\
\hline Dot Size $[\mu \mathrm{m}]$ & Slope $\left[\mathbf{F}^{-2} \mathbf{v}\right]$ & $\mathbf{C}_{\text {jo }}^{-\mathbf{2}}\left[\mathbf{F}^{-2}\right]$ & $\begin{array}{l}\text { Carrier } \\
\text { Conc. }\left[\mathbf{c m}^{-3} \text { ] }\right.\end{array}$ & $\begin{array}{l}\text { Built-in } \\
\text { Voltage [V] }\end{array}$ \\
\hline 200 & $-5.68 \times 10^{18}$ & $5.08 \times 10^{18}$ & $1.31 \times 10^{18}$ & 0.92 \\
\hline 190 & $-5.83 \times 10^{18}$ & $6.40 \times 10^{18}$ & $1.57 \times 10^{18}$ & 1.12 \\
\hline 180 & $-8.23 \times 10^{18}$ & $7.50 \times 10^{18}$ & $1.38 \times 10^{18}$ & 0.94 \\
\hline 170 & $-10.34 \times 10^{18}$ & $9.56 \times 10^{18}$ & $1.37 \times 10^{18}$ & 0.95 \\
\hline 160 & $-13.13 \times 10^{18}$ & $12.20 \times 10^{18}$ & $1.38 \times 10^{18}$ & 0.96 \\
\hline 150 & $-16.64 \times 10^{18}$ & $15.44 \times 10^{18}$ & $1.41 \times 10^{18}$ & 0.95 \\
\hline
\end{tabular}

Mean Carrier Concentration $=1.407 \times 10^{18} \mathrm{~cm}^{-3}$

Mean Built-in Voltage $=0.973 \mathrm{~V}$

Table 6.2: Measurement between a dot and an Ohmic contact on backside of GaAs.

\begin{tabular}{|c|c|c|c|c|}
\hline \multicolumn{6}{|c|}{ Measurement between a dot and Ohmic contact on backside } \\
\hline Dot Size $[\mu \mathrm{m}]$ & Slope $\left[\mathbf{F}^{-2} \mathbf{v}\right]$ & $\mathbf{C}_{\text {jo }}{ }^{-2}\left[\mathbf{F}^{-2}\right]$ & $\begin{array}{l}\text { Carrier } \\
\text { Conc. }\left[\mathbf{c m}^{-3} \text { ] }\right.\end{array}$ & $\begin{array}{l}\text { Built-in } \\
\text { Voltage [V] }\end{array}$ \\
\hline 200 & $-5.03 \times 10^{18}$ & $5.05 \times 10^{18}$ & $1.50 \times 10^{18}$ & 1.03 \\
\hline 190 & $-5.71 \times 10^{18}$ & $6.15 \times 10^{18}$ & $1.60 \times 10^{18}$ & 1.10 \\
\hline 180 & $-7.50 \times 10^{18}$ & $7.50 \times 10^{18}$ & $1.50 \times 10^{18}$ & 1.03 \\
\hline 170 & $-9.60 \times 10^{18}$ & $9.60 \times 10^{18}$ & $1.48 \times 10^{18}$ & 1.03 \\
\hline 160 & $-12.35 \times 10^{18}$ & $12.21 \times 10^{18}$ & $1.47 \times 10^{18}$ & 1.01 \\
\hline 150 & $-15.78 \times 10^{18}$ & $15.50 \times 10^{18}$ & $1.50 \times 10^{18}$ & 1.01 \\
\hline 140 & $-20.97 \times 10^{18}$ & $20.40 \times 10^{18}$ & $1.48 \times 10^{18}$ & 1.00 \\
\hline
\end{tabular}

Mean Carrier Concentration $=1.504 \times 10^{18} \mathrm{~cm}^{-3}$

Mean Built-in Voltage $=1.03 \mathrm{~V}$ 


\section{Appendix E-DLTS: Brief Discussion}

An extension of the $\mathrm{C}-\mathrm{V}$ analysis is the technique known as deep-level transient spectroscopy (DLTS), invented in the 70's by D. V. Lang [10]. This technique consists of applying a pulse to the depletion layer of a semiconductor and measuring the transient in capacitance due to the presence of traps. The depletion layer is kept at a quiescent reverse biased and a pulse is then applied to change the carrier occupancy in the traps. As a consequence of this change in charge, the capacitance of the junction goes from a steady state value to a transient due to the occupancy of the traps and then returns to its steady state value when the population of carriers returns to equilibrium. This sequence of occupation of traps is illustrated by figure 6.1, and the transient due to this occupation is illustrated in figure 6.2.
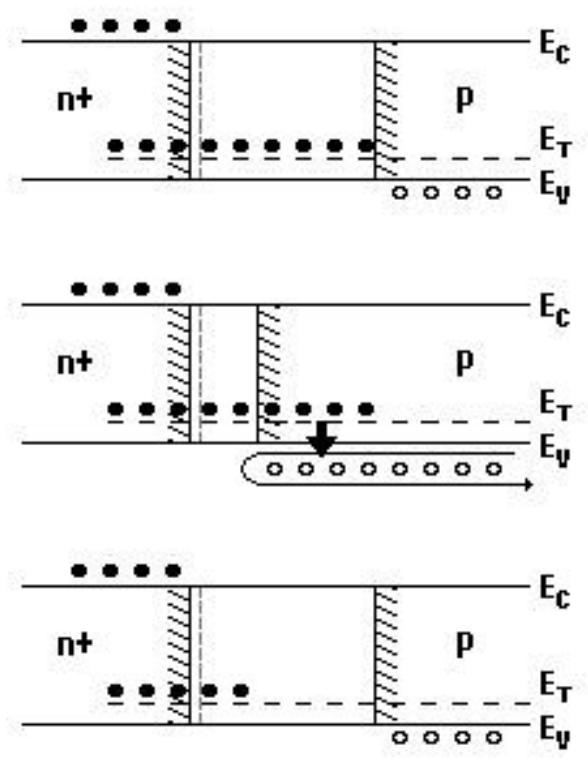

Figure 6.1: Sequence of occupation of majority-carrier traps. 


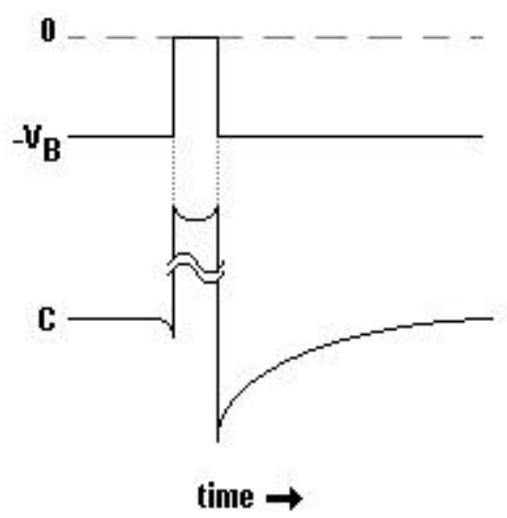

Figure 6.2: Capacitance transient of a junction due to an applied pulse.

The normalized DLTS signal, defined as $\mathrm{S}(\mathrm{T})$, is given by equation 6.1a. In this equation $\mathrm{C}\left(\mathrm{t}_{\mathrm{x}}\right)$ is the capacitance at the instant $\mathrm{t}_{\mathrm{x}}$ of the transient, and ?C(0) is the capacitance change due to the beginning of the pulse.

$$
S(T)=\frac{\left[C\left(t_{1}\right)-C\left(t_{2}\right)\right]}{\Delta C(0)}
$$

Assuming that the decay of the transient has an exponential shape, equation 6.1a can be rewritten as equation $6.1 \mathrm{~b}$. In this last equation, $\mathrm{t}$ is the emission rate of the trap.

$$
S(T)=\exp \left(-\frac{t_{1}}{\tau}\right)-\exp \left(-\frac{t_{2}}{\tau}\right)
$$

Taking the derivative of equation $6.1 \mathrm{~b}$ with respect to the emission rate, one can find the maximum emission rate, as shown in equation 6.1c.

$$
\tau_{\max }=\left(t_{1}-t_{2}\right) \cdot\left[\ln \left(\frac{t_{1}}{t_{2}}\right)\right]^{-1}
$$


A set of measurements can be performed at various temperatures in order to find a profile for the normalized DLTS signal. A typical set of curves for this measurement is shown in figure 6.3 .

The time window for each of these measurements at different temperatures can be changed so that a plot of the normalized DLTS signal can be constructed. A typical plot of the normalized DLTS signal is shown in figure 6.4.
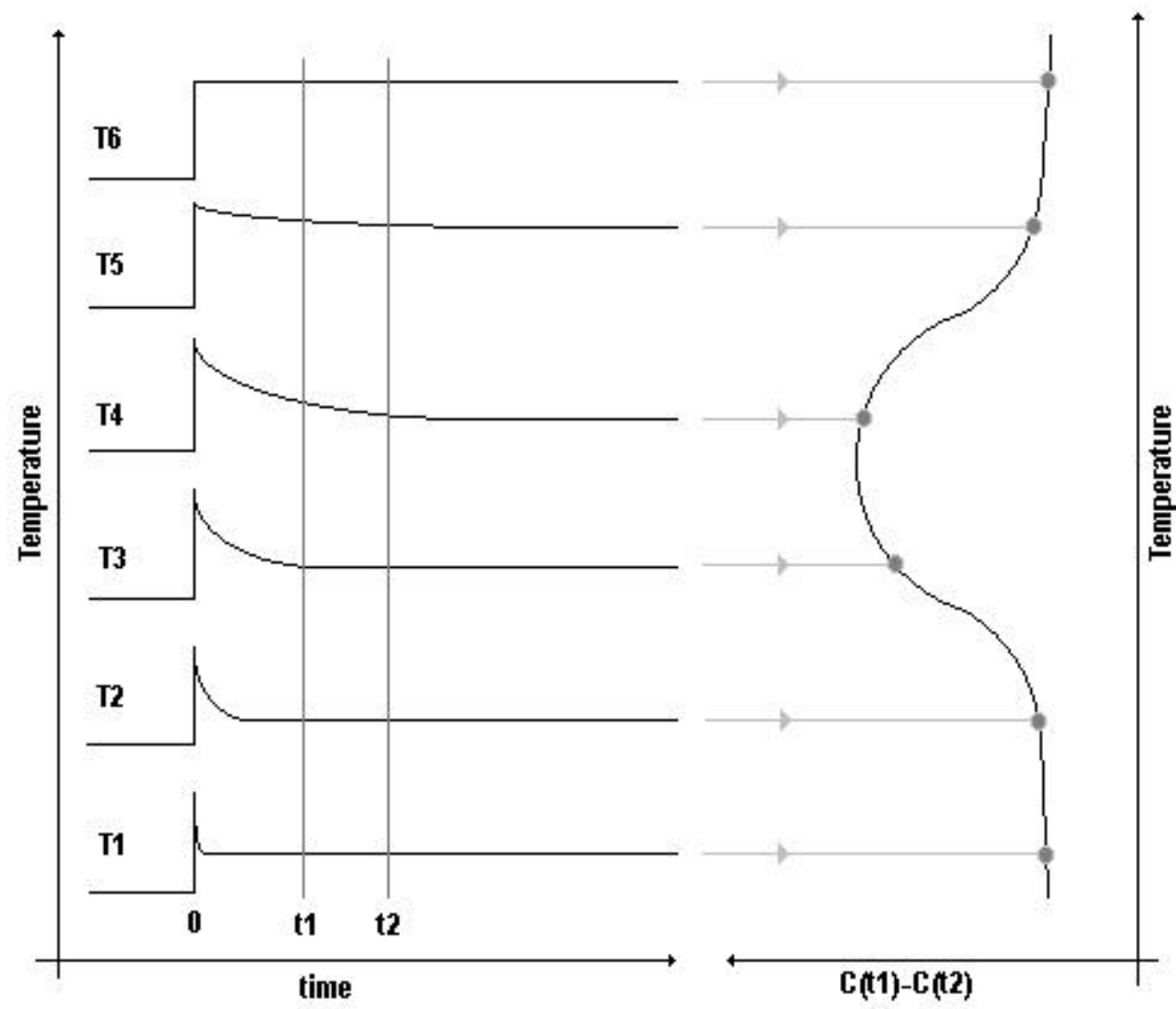

Figure 6.3: DLTS Transients at different temperatures with a fixed time window. 


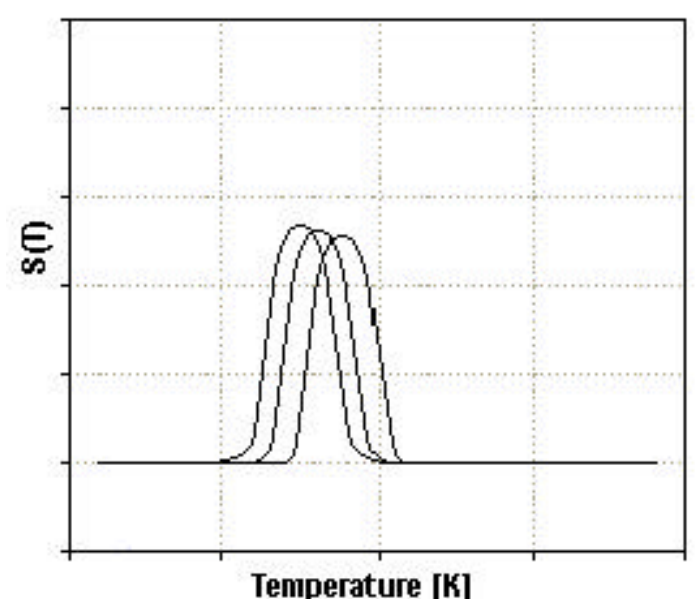

Figure 6.4: Typical plot of the normalized DLTS signal versus temperature.

Applying equation 6.1c to the plot of $\mathrm{S}(\mathrm{T})$ one can find the maximum emission rate for each of the time windows for a particular trap and thus construct a plot of thermal emission rates versus temperature. The expression that relates the thermal emission rate to the temperature is given in equation 6.2 [10], and can be used to find energy of an individual trap. In this equation $\mathrm{K}$ is a constant, and e is the thermal emission rate.

$$
e=K \cdot \exp \left(-\frac{\Delta E}{k_{B} T}\right)
$$

If one takes a logarithmic plot of equation 6.2 versus the inverse of the temperature, the energy of the trap is simply the slope of the graph divided by the Boltzmann constant. This last procedure is visualized in figure 6.5. 


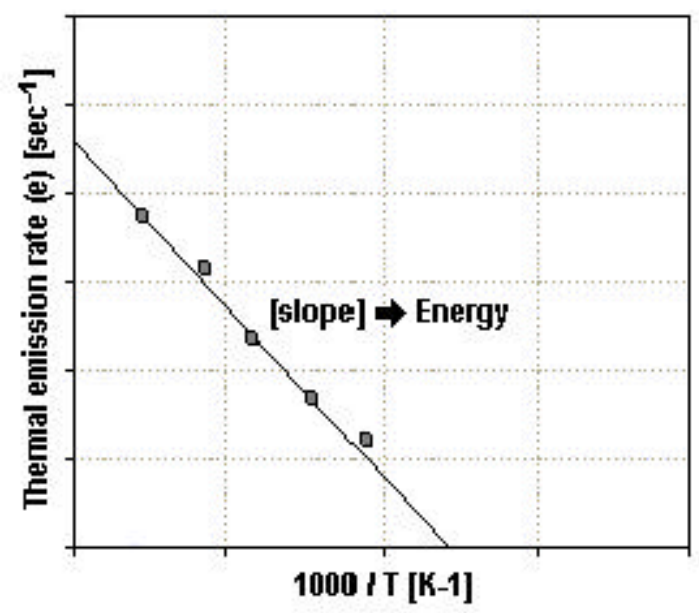

Figure 6.5: Typical log plot of the thermal emission rate versus 1000/T.

Unlike the previous measurements, DLTS requires three different instruments. The first is a HP8112A - Pulse Generator, the second is a LakeShore 330 - Temperature Controller, and the third is a Boonton 7200 - Capacitance Meter. These three equipments are connected to a data acquisition board National Instruments PC-MIO-16E-4 and are controlled via GPIB by a program written in LabView. The general connection among these instruments is shown in figure 6.6.

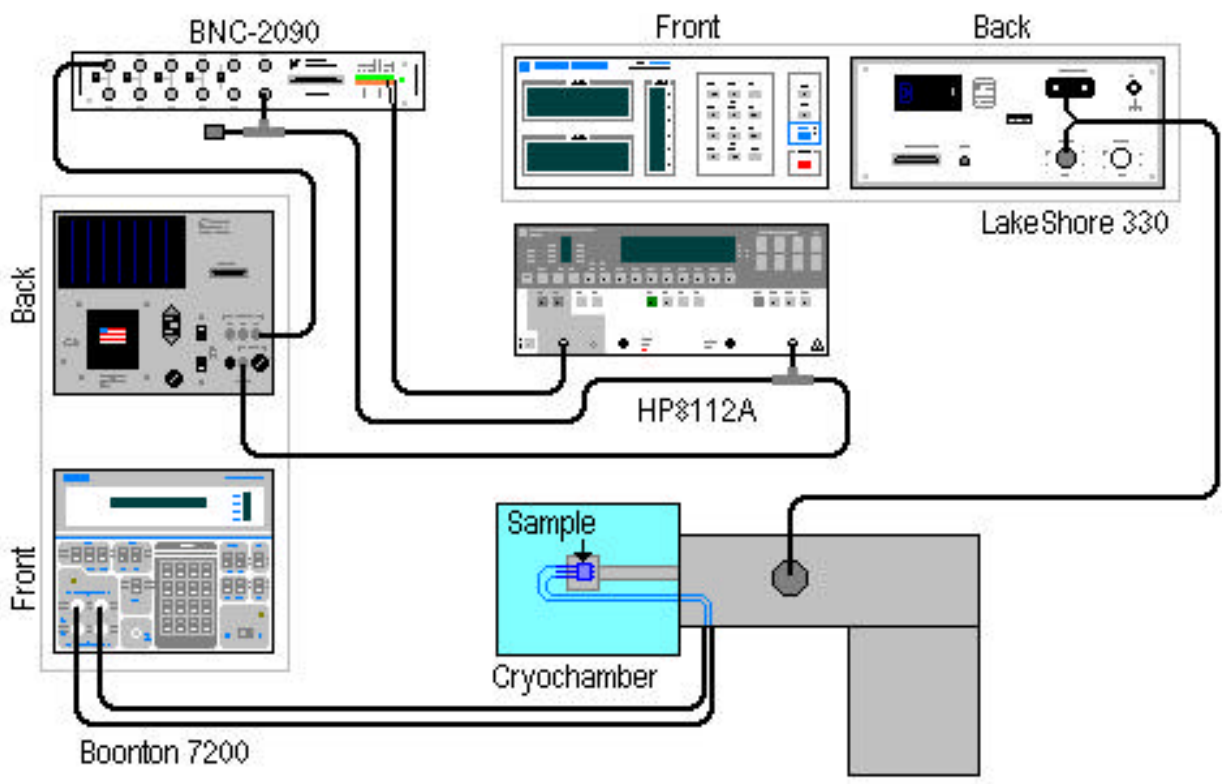

Figure 6.6: General connection among instruments for DLTS measurements. 
The principle of operation of the DLTS setup is the following. The computer sends a signal to the data acquisition board asking for a timed pulse. When this pulse starts, pin CTR0OUT on the BNC-2090 goes high with respect to the digital ground DGND1. This pulse starts a programmed pulse on the HP8112A through connector EXT INPUT. The resulting pulse is sent by OUTPUT to the BOONTON7200 to create a bias pulse in the DUT. This signal is also sent to pin PFI0/TRIG1 on the BNC-2090. The pulse is sent back to the data acquisition board with the purpose of creating a hardware triggering. Thus, every time the signal on that pin goes high, a new acquisition is initiated by pin ACH0 also on BNC-2090. This last pin is connected to the pin CAP on the BOONTON8112A where the output capacitance is produced.

A program created in LabView (listed ahead) controls the operation of the setup the following way. First a particular temperature is set in LakeShore330. When the temperature is within an acceptable range of stabilization, the program sends a pulse to the setup and a new acquisition is started. The duration of the transient can be programmed, and the average of a number of transients is then stored in a file. After the program sweeps all the temperatures, the whole spectrum can be saved in a file. Also, the standard deviation resulted from that averaging is saved in another file.

Both files can be imported in a graphics manipulator program such as SigmaPlot for data analysis. The standard deviation program contains a column for the temperature, and another column for the standard deviation resulted from the measurements of transient taken at that temperature. A curve of standard deviation versus temperature can be plotted to show how good the measurement was. A typical plot is shown on figure 6.7. 


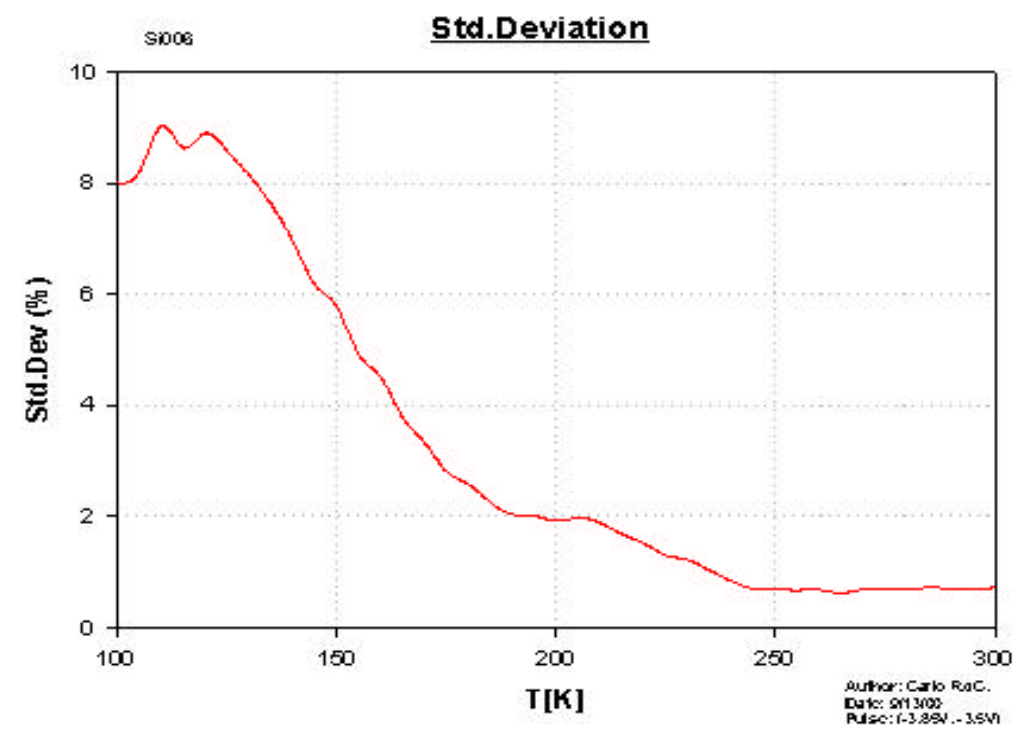

Figure 6.7: Typical plot of standard deviation versus temperature for a DLTS measurement.

The file that contains the transients has an individual row for each of the transients. After the file is imported, the whole matrix can be transposed to have columns representing the temperatures, and rows representing the time. The transients can then be plotted in a different graph. A typical plot of the transients is shown in figure 6.8.

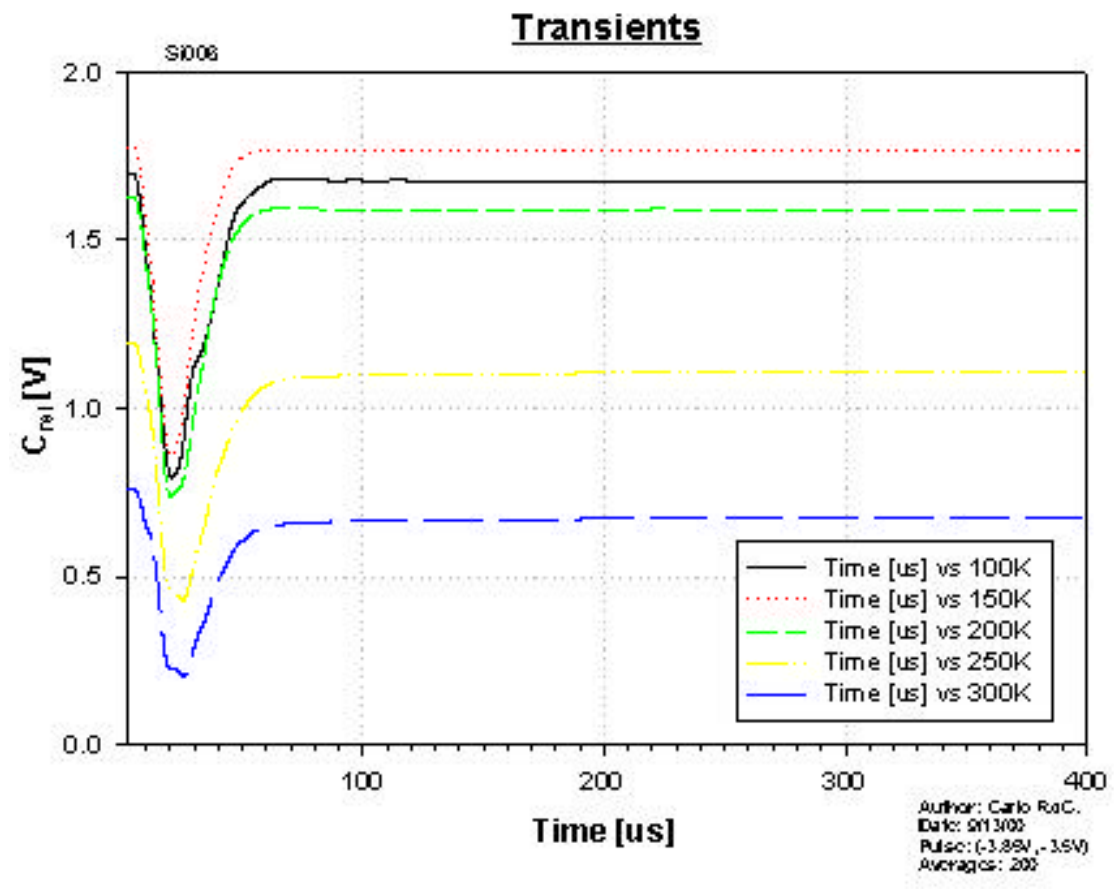

Figure 6.8: Transients in a typical DLTS signal. 
Attention should be taken to the fact that the capacitance is given by the equivalent voltage provided by the equipment. This voltage does not need to be converted to a value of capacitance, as it is going to become clear as this explanation develops.

Given the plot of transients, several time windows are chosen and plots of the relative capacitance (capacitance at the initial time of the window subtracted from the capacitance at the final time of the window) versus temperature can be made. A typical plot of the relative capacitance at different time windows versus temperature is shown in figure 6.9.

Analyzing the plot of relative capacitance versus temperature, one can find the position of the maximums and fit them with equation 6.2 to find the energy of each of the traps in the device under test.

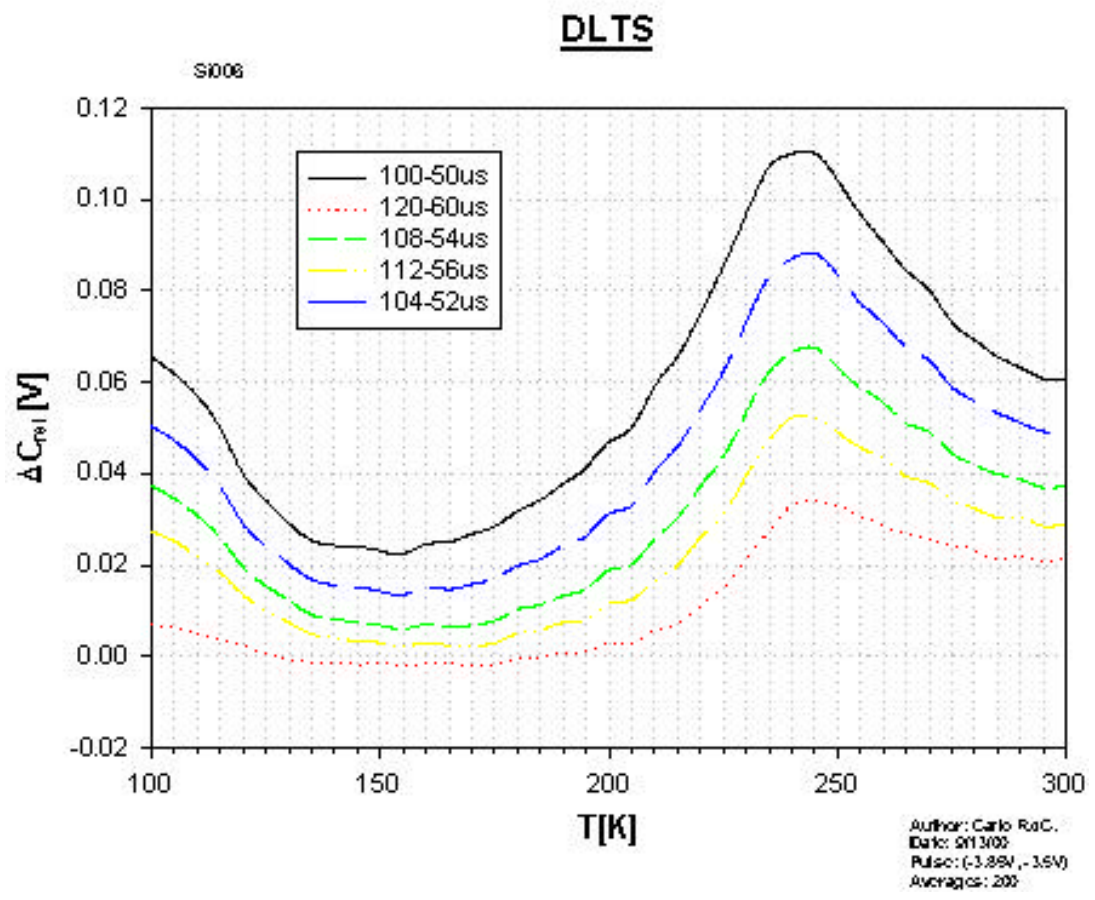

Figure 6.9: Typical relative capacitances versus temperature in a DLTS measurement. 


\section{DLTS.VI:}

\section{Front Panel}

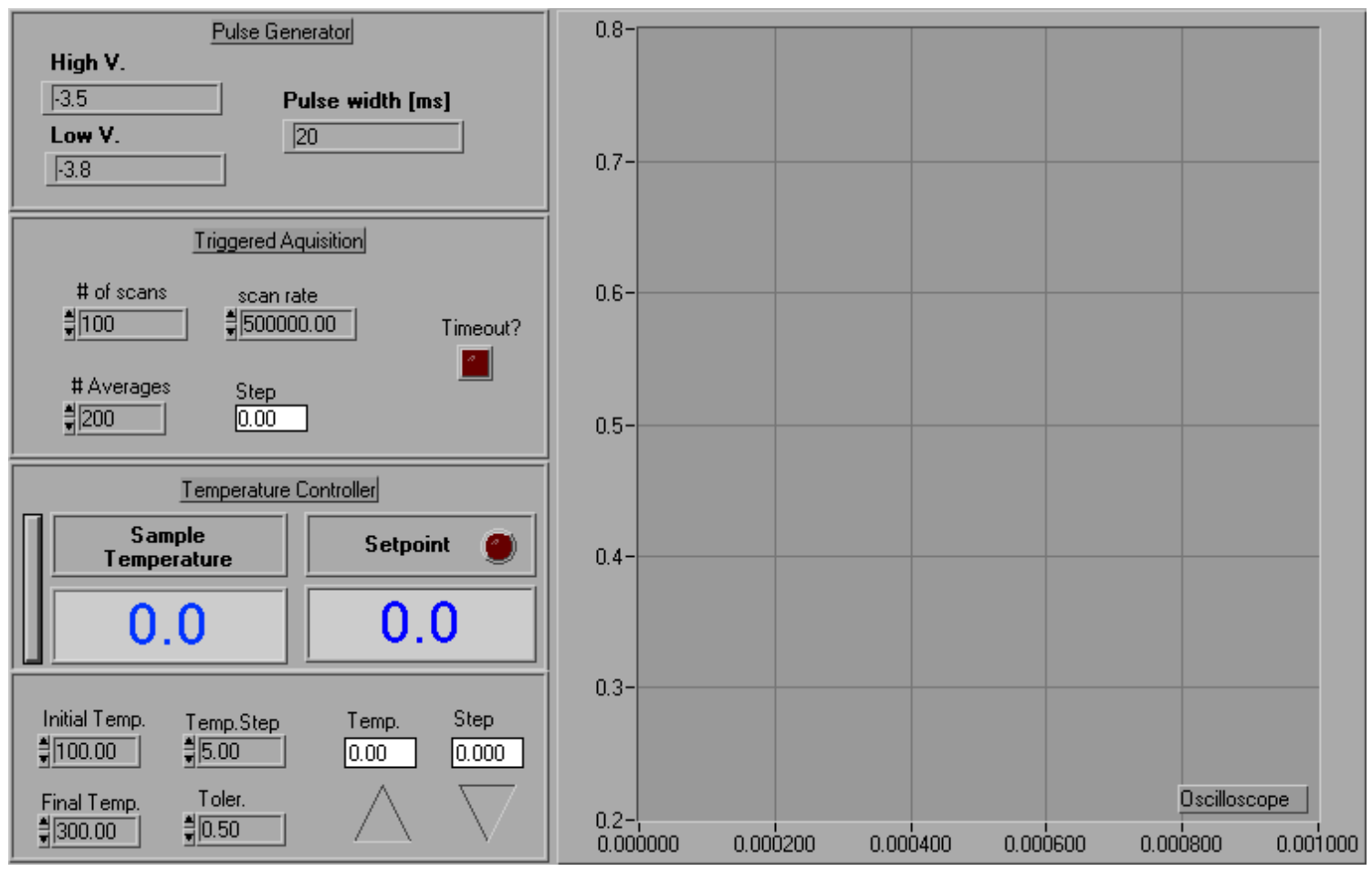

\section{Block Diagram}

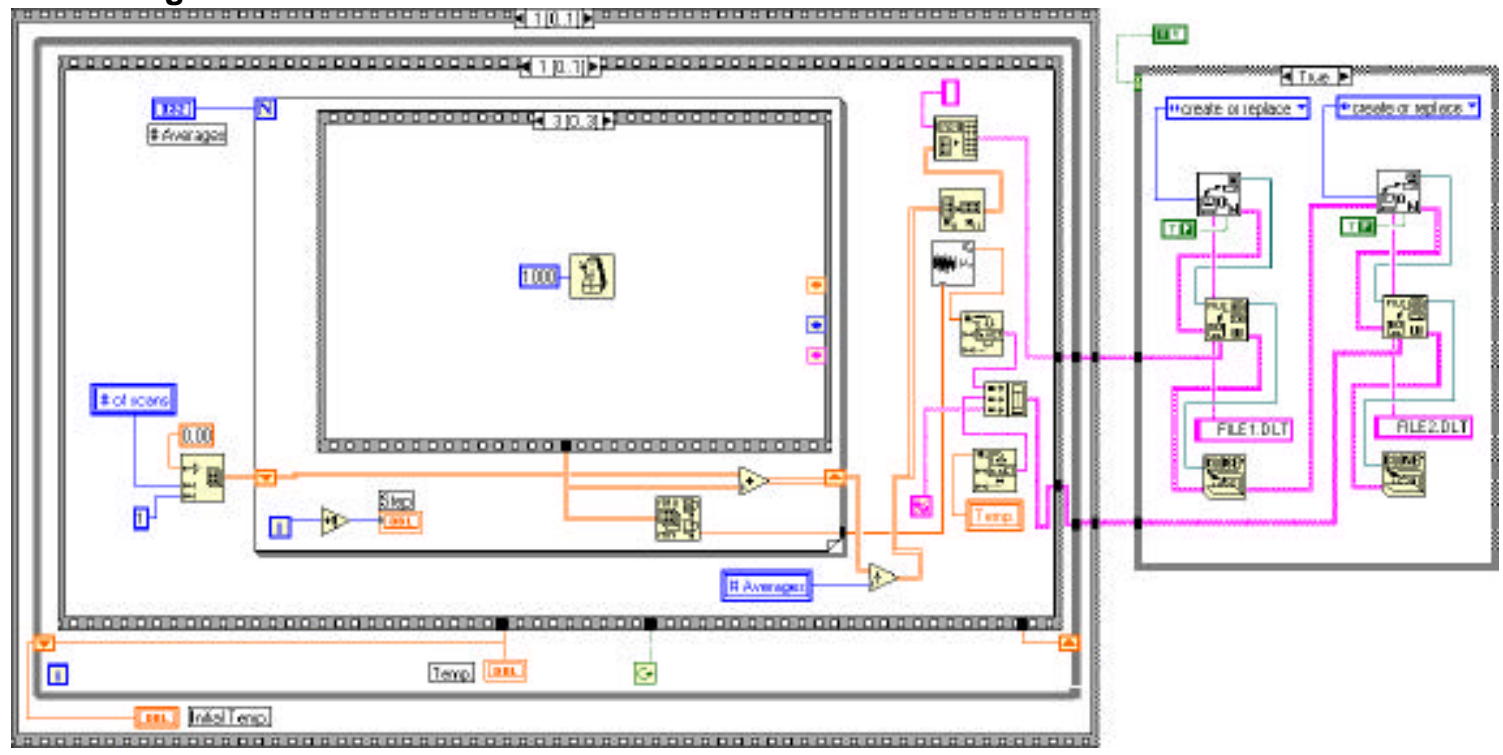




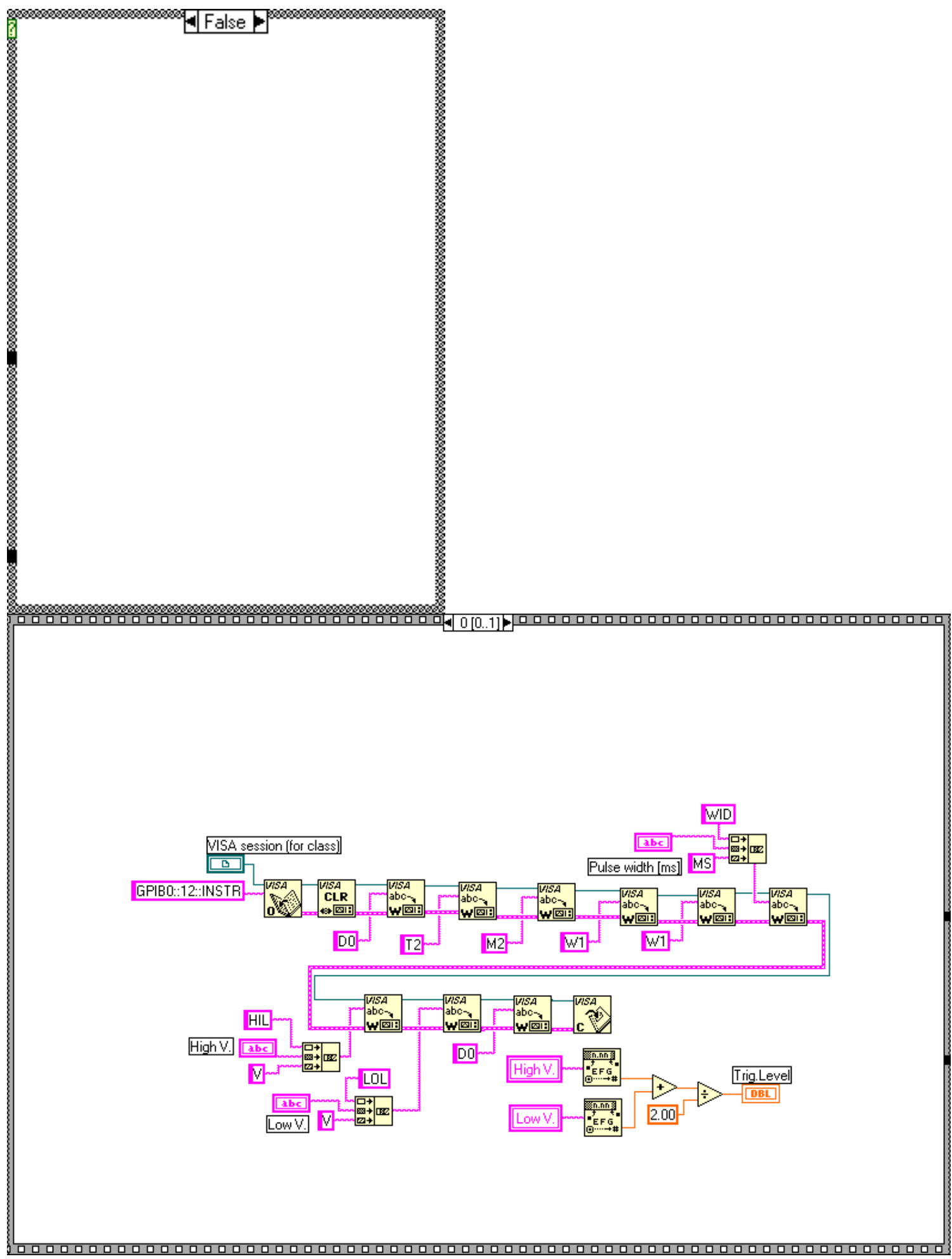




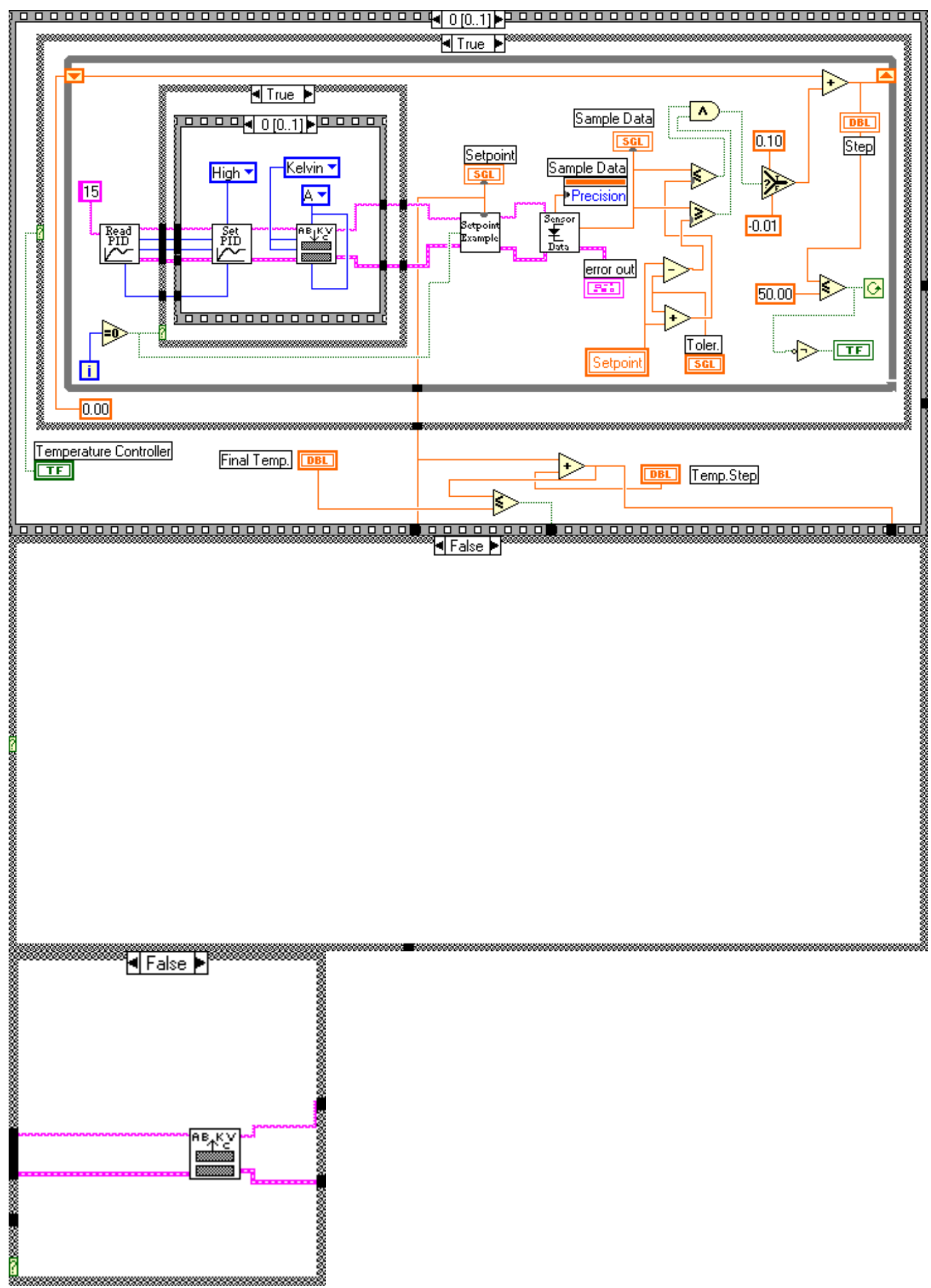




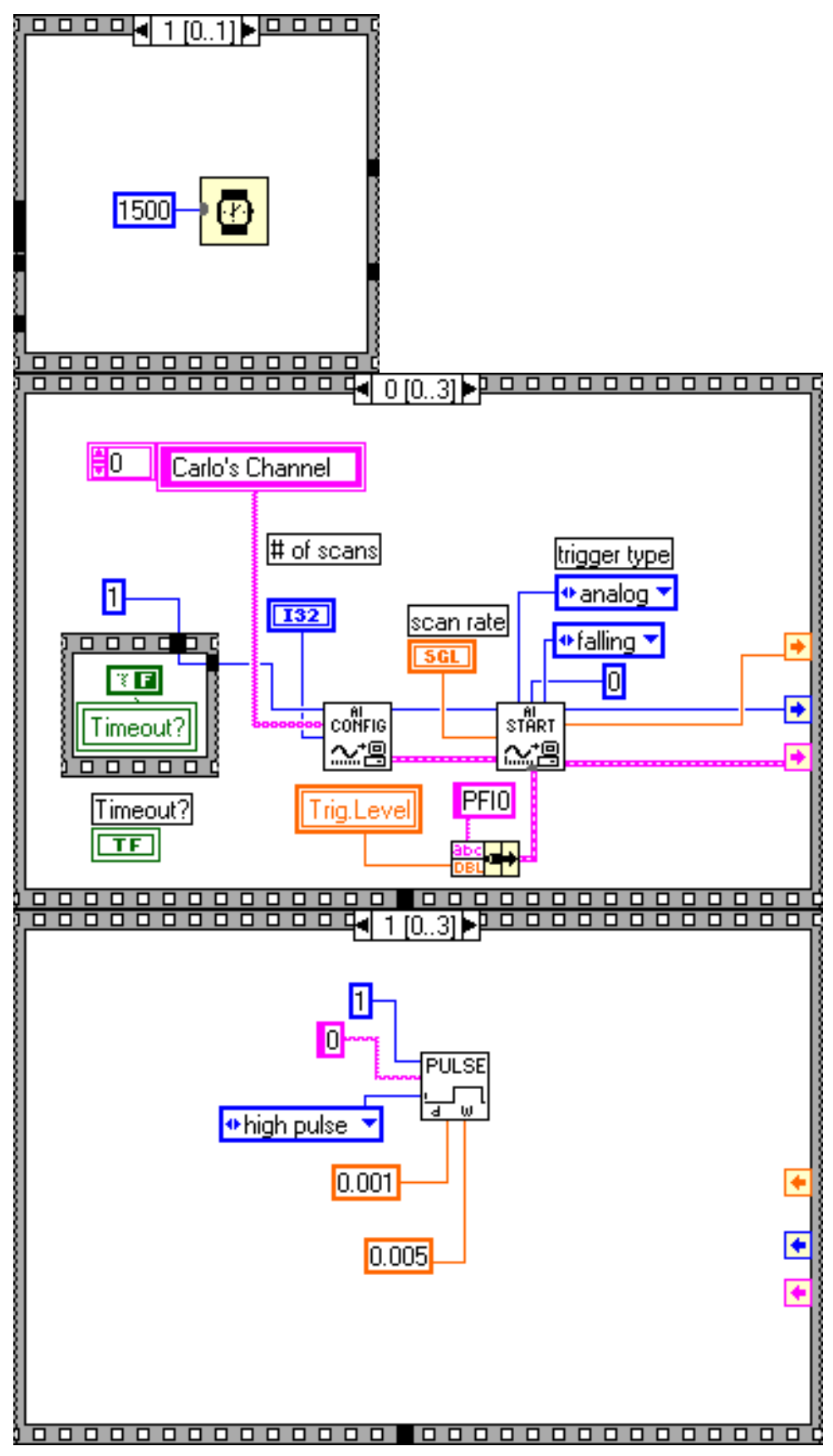




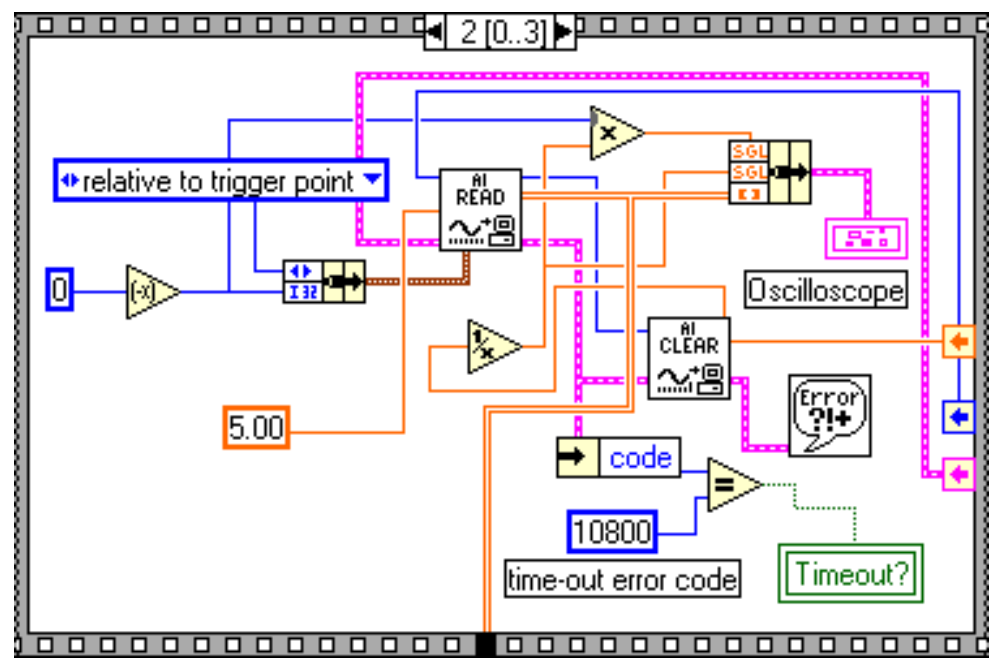




\section{Appendix F - Hall Measurements: Brief Discussion}

This section briefly explains the Hall measurements performed on the studied samples. For a more detailed and complete explanation, please see references [25-28]. The samples used in this study had a square Van Der Pauw geometry [25], and in order to perform Hall measurement, Ohmic contacts on their corners needed to be placed. These contacts were created using indium. Figure 6.10 shows the geometry of a typical sample with contacts on its corners. The sample is loaded in a chamber, and the contacts are connected to a switching board (Keithley 7001). This switching board is connected to a current source (Keithley 220), a picoammeter (Keithley 485) and a voltage meter (Keithley 196) so that all combinations of resistances can be measured by dividing the measured voltage by the applied current. This configuration is also shown in figure 6.10 . The switching board is controlled by an IBM486PC via IEEE-488BUS. The chamber loaded with the sample is placed in a Varian water-cooled electro-magnet that uses a HP 6465B power supply. This magnet has a typical magnetic field of 6 kgauss.

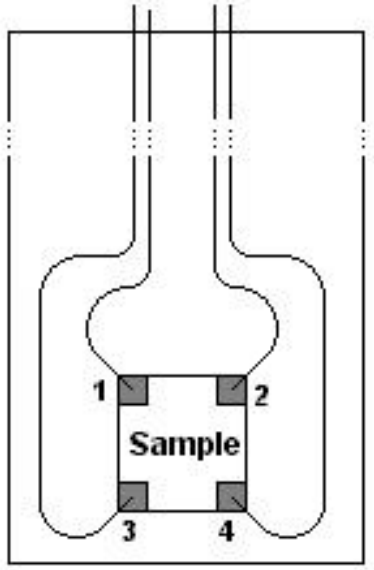

Front View

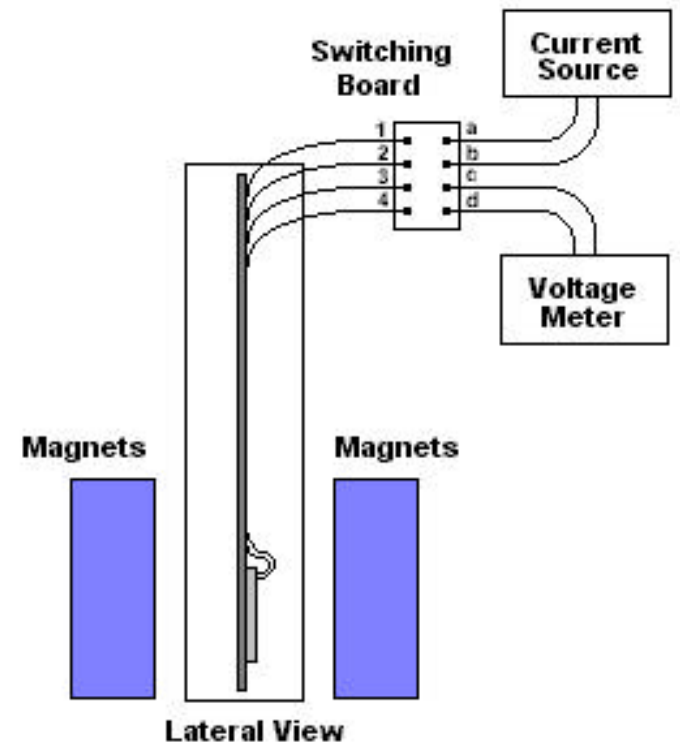

Figure 6.10: Typical sample with contacts on its corners, and connections diagram. 
A set of resistances is then measured by making the appropriate connections in the switching board. These resistances are defined as shown in table 6.3.

Table 6.3: Definition of resistances for Hall measurement.

\begin{tabular}{|l|l|}
\hline$R_{21,34}=V_{34} / I_{21}$ & $R_{12,43}=V_{43} / I_{12}$ \\
\hline$R_{32,41}=V_{41} / I_{32}$ & $R_{23,14}=V_{14} / I_{23}$ \\
\hline$R_{43,12}=V_{12} / I_{43}$ & $R_{34,21}=V_{21} / I_{34}$ \\
\hline$R_{14,23}=V_{23} / I_{14}$ & $R_{41,32}=V_{32} / I_{41}$ \\
\hline
\end{tabular}

The sheet resistance of the sample can be determined by finding two characteristic resistances. The definitions of these resistances are shown in table 6.4.

Table 6.4: Definition of characteristic resistances.

$$
\begin{aligned}
& R_{\mathrm{A}}=\left(R_{21,34}+R_{12,43}+R_{43,12}+R_{34,21}\right) / 4 \\
& R_{\mathrm{B}}=\left(R_{32,41}+R_{23,14}+R_{14,23}+R_{41,32}\right) / 4
\end{aligned}
$$

Then the Van der Pauw equation (Equation 6.3) is solved numerically in order to find the sheet resistance of the material $\left(\mathrm{R}_{\mathrm{S}}\right)$.

$$
\exp \left(-\pi R_{\mathrm{A}} / R_{\mathrm{S}}\right)+\exp \left(-\pi R_{\mathrm{B}} / R_{\mathrm{S}}\right)=1
$$

If the thickness of the sample is known, then the resistivity $(\rho)$ of the material can be found by applying equation 6.4 .

$$
\mathrm{R}_{\mathrm{S}}=\rho / \mathrm{t}_{\mathrm{k}}
$$

The next step is to find the sheet carrier density. This can be calculated if, first, a magnetic induction (B) is applied to the sample. Then and current is applied between contacts 1 and 3, 3 and 1, 2 and 4, and 4 and 2 to find the Hall voltages $V_{24}, V_{42}, V_{31}$, and $\mathrm{V}_{13}$. Combining these voltages with the applied currents, one can find the equivalent resistances $R_{24,13}, R_{42,31}, R_{31,24}, R_{13,42}$. The Hall mobility can be found by applying equation 6.5 to this set of resistances. 


$$
\mu_{H}=\frac{t_{K}}{B} \cdot \frac{R_{24,13}+R_{42,31}+R_{31,24}+R_{13,42}}{4 \cdot \rho}
$$

Having the Hall mobility, one can find the carrier concentration by using equation 6.6 .

$$
(n, p)_{H}=\frac{1}{\mu_{H} \cdot \rho \cdot q}
$$

The results obtained from samples GaN9906E, GaN9920, and GaAs test sample are shown on tables $6.5-6.16$. In these tables, the first eight voltages are the voltages used to calculate the resistivity of the material. The next four voltages are used to calculate the mobility and carrier concentration, as explained above.

Table 6.5: Hall results for GaN9906E at $100 \times 10^{-6} \mathrm{~A}$.

\begin{tabular}{|c|c|}
\hline \multicolumn{2}{|c|}{ GaN9906E } \\
\hline \multicolumn{2}{|c|}{} \\
\hline Voltage \#1: & $-0.224422 \mathrm{~V}$ \\
\hline Voltage \#2: & $0.224968 \mathrm{~V}$ \\
\hline Voltage \#3: & $-3.576766 \mathrm{~V}$ \\
\hline Voltage \#4: & $3.579535 \mathrm{~V}$ \\
\hline Voltage \#5: & $-0.225319 \mathrm{~V}$ \\
\hline Voltage \#6: & $0.225533 \mathrm{~V}$ \\
\hline Voltage \#7: & $-3.586768 \mathrm{~V}$ \\
\hline Voltage \#8: & $3.592235 \mathrm{~V}$ \\
\hline Voltage \#9: & $-3.457655 \mathrm{~V}$ \\
\hline Voltage \#10: & $3.458045 \mathrm{~V}$ \\
\hline Voltage \#11: & $3.292646 \mathrm{~V}$ \\
\hline Voltage \#12: & $-3.294551 \mathrm{~V}$ \\
\hline & $100 \times 10^{-6} \mathrm{~A}$ \\
\hline Current: & $0.000200 \mathrm{~cm}^{-1}$ \\
\hline Thickness: & $10.765 \Omega-\mathrm{cm}^{-1}-\mathrm{Sec}^{-1}$ \\
\hline Resistivity: & $254.29 \mathrm{~cm}^{2}-\mathrm{V}^{-1} \mathrm{~cm}^{-3}$ \\
\hline Mobility: & $-2.28 \times 10^{15}$ \\
\hline Density: &
\end{tabular}


Table 6.6: Hall results for GaN9906E at $50 \times 10^{-6} \mathrm{~A}$.

\begin{tabular}{|c|c|}
\hline \multicolumn{2}{|c|}{ GaN9906E } \\
\hline \multicolumn{2}{|c|}{} \\
\hline Voltage \#1: & $-0.114122 \mathrm{~V}$ \\
\hline Voltage \#2: & $0.114296 \mathrm{~V}$ \\
\hline Voltage \#3: & $-1.820468 \mathrm{~V}$ \\
\hline Voltage \#4: & $1.821673 \mathrm{~V}$ \\
\hline Voltage \#5: & $-0.114440 \mathrm{~V}$ \\
\hline Voltage \#6: & $0.114516 \mathrm{~V}$ \\
\hline Voltage \#7: & $-1.824763 \mathrm{~V}$ \\
\hline Voltage \#8: & $1.826497 \mathrm{~V}$ \\
\hline Voltage \#9: & $-1.757337 \mathrm{~V}$ \\
\hline Voltage \#10: & $1.757763 \mathrm{~V}$ \\
\hline Voltage \#11: & $1.673118 \mathrm{~V}$ \\
\hline Voltage \#12: & $-1.674004 \mathrm{~V}$ \\
\hline & \\
\hline Current: & $50 \times 10^{-6} \mathrm{~A}$ \\
\hline Thickness: & $0.000200 \mathrm{~cm}^{-6}$ \\
\hline Resistivity: & $10.949 \Omega-\mathrm{cm}^{2}$ \\
\hline Mobility: & $255.69 \mathrm{~cm}^{2}-\mathrm{V}^{-1}-\mathrm{Sec}^{-1}$ \\
\hline Density: & $-2.23 \times 10^{15} \mathrm{~cm}^{-3}$ \\
\hline
\end{tabular}

Table 6.7: Hall results for GaN9906E at $30 \times 10^{-6} \mathrm{~A}$.

\begin{tabular}{|c|c|}
\hline \multicolumn{2}{|c|}{ GaN9906E } \\
\hline \multicolumn{2}{|c|}{} \\
\hline Voltage \#1: & $-0.069198 \mathrm{~V}$ \\
\hline Voltage \#2: & $0.069258 \mathrm{~V}$ \\
\hline Voltage \#3: & $-1.104211 \mathrm{~V}$ \\
\hline Voltage \#4: & $1.104678 \mathrm{~V}$ \\
\hline Voltage \#5: & $-0.069310 \mathrm{~V}$ \\
\hline Voltage \#6: & $0.069338 \mathrm{~V}$ \\
\hline Voltage \#7: & $-1.105752 \mathrm{~V}$ \\
\hline Voltage \#8: & $1.106387 \mathrm{~V}$ \\
\hline Voltage \#9: & $-1.063945 \mathrm{~V}$ \\
\hline Voltage \#10: & $1.064106 \mathrm{~V}$ \\
\hline Voltage \#11: & $1.012578 \mathrm{~V}$ \\
\hline Voltage \#12: & $-1.012901 \mathrm{~V}$ \\
\hline & \\
\hline Current: & $30 \times 10^{-6} \mathrm{~A}$ \\
\hline Thickness: & $0.000200 \mathrm{~cm}^{-1}$ \\
\hline Resistivity: & $11.060 \Omega-\mathrm{cm}^{-1}$ \\
\hline Mobility: & $257.61 \mathrm{~cm}^{2}-\mathrm{V}^{-1}-\mathrm{Sec}^{-1}$ \\
\hline Density: & $-2.19 \times 10^{15} \mathrm{~cm}^{-3}$ \\
\hline
\end{tabular}


Table 6.8: Hall results for GaN9906E at $10 \times 10^{-6} \mathrm{~A}$.

\begin{tabular}{|c|c|}
\hline \multicolumn{2}{|c|}{ GaN9906E } \\
\hline \multicolumn{2}{|c|}{} \\
\hline Voltage \#1: & $-0.022054 \mathrm{~V}$ \\
\hline Voltage \#2: & $0.022083 \mathrm{~V}$ \\
\hline Voltage \#3: & $-0.350951 \mathrm{~V}$ \\
\hline Voltage \#4: & $0.351407 \mathrm{~V}$ \\
\hline Voltage \#5: & $-0.022161 \mathrm{~V}$ \\
\hline Voltage \#6: & $0.022180 \mathrm{~V}$ \\
\hline Voltage \#7: & $-0.352693 \mathrm{~V}$ \\
\hline Voltage \#8: & $0.353166 \mathrm{~V}$ \\
\hline Voltage \#9: & $-0.340152 \mathrm{~V}$ \\
\hline Voltage \#10: & $0.340508 \mathrm{~V}$ \\
\hline Voltage \#11: & $0.324209 \mathrm{~V}$ \\
\hline Voltage \#12: & $-0.324560 \mathrm{~V}$ \\
\hline & $10 \times 10^{-6} \mathrm{~A}$ \\
\hline Current: & $0.000200 \mathrm{~cm}^{-1}$ \\
\hline Thickness: & $10.577 \Omega_{-\mathrm{cm}}$ \\
\hline Resistivity: & $251.26 \mathrm{~cm}^{2}-\mathrm{V}^{-1}-\mathrm{Sec}^{-1}$ \\
\hline Mobility: & $-2.35 \times 10^{15} \mathrm{~cm}^{-3}$ \\
\hline Density: &
\end{tabular}

Table 6.9: Hall results for GaN9920 at $5 \times 10^{-6} \mathrm{~A}$.

\begin{tabular}{|c|c|}
\hline \multicolumn{2}{|c|}{ GaN9920 } \\
\hline \multicolumn{2}{|c|}{} \\
\hline Voltage \#1: & $-0.309388 \mathrm{~V}$ \\
\hline Voltage \#2: & $0.310110 \mathrm{~V}$ \\
\hline Voltage \#3: & $-0.099514 \mathrm{~V}$ \\
\hline Voltage \#4: & $0.099783 \mathrm{~V}$ \\
\hline Voltage \#5: & $-0.312176 \mathrm{~V}$ \\
\hline Voltage \#6: & $0.312804 \mathrm{~V}$ \\
\hline Voltage \#7: & $-0.100385 \mathrm{~V}$ \\
\hline Voltage \#8: & $0.100565 \mathrm{~V}$ \\
\hline Voltage \#9: & $0.207544 \mathrm{~V}$ \\
\hline Voltage \#10: & $-0.207833 \mathrm{~V}$ \\
\hline Voltage \#11: & $-0.221987 \mathrm{~V}$ \\
\hline Voltage \#12: & $0.222300 \mathrm{~V}$ \\
\hline & $5 \times 10^{-6} \mathrm{~A}$ \\
\hline Current: & $0.000208 \mathrm{~cm}^{-1}$ \\
\hline Thickness: & $34.949 \Omega-\mathrm{cm}^{-1}-\mathrm{Sec}^{-1}$ \\
\hline Resistivity: & $143.38 \mathrm{~cm}^{2}-\mathrm{V}^{-1} \mathrm{~cm}^{-3}$ \\
\hline Mobility: & $-1.25 \times 10^{15}$ \\
\hline Density: & \\
\hline
\end{tabular}


Table 6.10: Hall results for GaN9920 at $0.5 \times 10^{-6} \mathrm{~A}$.

\begin{tabular}{|c|c|}
\hline \multicolumn{2}{|c|}{ GaN9920 } \\
\hline \multicolumn{2}{|c|}{} \\
\hline Voltage \#1: & $-0.031924 \mathrm{~V}$ \\
\hline Voltage \#2: & $0.031962 \mathrm{~V}$ \\
\hline Voltage \#3: & $-0.010257 \mathrm{~V}$ \\
\hline Voltage \#4: & $0.010269 \mathrm{~V}$ \\
\hline Voltage \#5: & $-0.032078 \mathrm{~V}$ \\
\hline Voltage \#6: & $0.032114 \mathrm{~V}$ \\
\hline Voltage \#7: & $-0.010303 \mathrm{~V}$ \\
\hline Voltage \#8: & $0.010318 \mathrm{~V}$ \\
\hline Voltage \#9: & $0.021234 \mathrm{~V}$ \\
\hline Voltage \#10: & $-0.021251 \mathrm{~V}$ \\
\hline Voltage \#11: & $-0.022670 \mathrm{~V}$ \\
\hline Voltage \#12: & $0.022694 \mathrm{~V}$ \\
\hline & $0.5 \times 10^{-6} \mathrm{~A}$ \\
\hline Current: & $0.000208 \mathrm{~cm}^{-1}$ \\
\hline Thickness: & $35.952 \Omega-\mathrm{cm}^{-1}$ \\
\hline Resistivity: & $138.80 \mathrm{~cm}^{2}-\mathrm{V}^{-1}-\mathrm{Sec}^{-1}$ \\
\hline Mobility: & $-1.25 \times 10^{15} \mathrm{~cm}^{-3}$ \\
\hline Density: &
\end{tabular}

Table 6.11: Hall results for GaN9920 at $50 \times 10^{-9} \mathrm{~A}$.

\begin{tabular}{|c|c|}
\hline \multicolumn{2}{|c|}{ GaN9920 } \\
\hline \multicolumn{2}{|c|}{} \\
\hline Voltage \#1: & $-0.003250 \mathrm{~V}$ \\
\hline Voltage \#2: & $0.003251 \mathrm{~V}$ \\
\hline Voltage \#3: & $-0.001044 \mathrm{~V}$ \\
\hline Voltage \#4: & $0.001044 \mathrm{~V}$ \\
\hline Voltage \#5: & $-0.003259 \mathrm{~V}$ \\
\hline Voltage \#6: & $0.003261 \mathrm{~V}$ \\
\hline Voltage \#7: & $-0.001044 \mathrm{~V}$ \\
\hline Voltage \#8: & $0.001049 \mathrm{~V}$ \\
\hline Voltage \#9: & $0.002150 \mathrm{~V}$ \\
\hline Voltage \#10: & $-0.002156 \mathrm{~V}$ \\
\hline Voltage \#11: & $-0.002293 \mathrm{~V}$ \\
\hline Voltage \#12: & $0.002299 \mathrm{~V}$ \\
\hline & \\
\hline Current: & $50 \times 10^{-9} \mathrm{~A}$ \\
\hline Thickness: & $0.000208 \mathrm{~cm}^{-9}$ \\
\hline Resistivity: & $36.543 \Omega-\mathrm{cm}^{2}$ \\
\hline Mobility: & $135.66 \mathrm{~cm}^{2}-\mathrm{V}^{-1}-\mathrm{Sec}^{-1}$ \\
\hline Density: & $-1.26 \times 10^{15} \mathrm{~cm}^{-3}$ \\
\hline
\end{tabular}


Table 6.12: Hall results for GaAs at $1 \times 10^{-2} \mathrm{~A}$.

\begin{tabular}{|c|c|}
\hline \multicolumn{2}{|c|}{ GaAs } \\
\hline Voltage \#1: & $-0.000124 \mathrm{~V}$ \\
\hline Voltage \#2: & $0.000125 \mathrm{~V}$ \\
\hline Voltage \#3: & $-0.000072 \mathrm{~V}$ \\
\hline Voltage \#4: & $0.000069 \mathrm{~V}$ \\
\hline Voltage \#5: & $-0.000126 \mathrm{~V}$ \\
\hline Voltage \#6: & $0.000122 \mathrm{~V}$ \\
\hline Voltage \#7: & $-0.000068 \mathrm{~V}$ \\
\hline Voltage \#8: & $0.000073 \mathrm{~V}$ \\
\hline Voltage \#9: & $-0.000002 \mathrm{~V}$ \\
\hline Voltage \#10: & $0.000002 \mathrm{~V}$ \\
\hline Voltage \#11: & $-0.000108 \mathrm{~V}$ \\
\hline Voltage \#12: & $0.000111 \mathrm{~V}$ \\
\hline Current: & $1 \times 10^{-2} \mathrm{~A}$ \\
\hline Thickness: & $0.0381 \mathrm{~cm}$ \\
\hline Resistivity: & $0.00163 \Omega-\mathrm{cm}$ \\
\hline Mobility: & $2167.83 \mathrm{~cm}^{2}-\mathrm{V}^{-1}-\mathrm{Sec}^{-1}$ \\
\hline Density: & $-1.76 \times 10^{18} \mathrm{~cm}^{-3}$ \\
\hline
\end{tabular}

Table 6.13: Hall results for GaAs at $5 \times 10^{-3} \mathrm{~A}$.

\begin{tabular}{|c|c|}
\hline \multicolumn{2}{|c|}{ GaAs } \\
\hline \multicolumn{2}{|c|}{} \\
\hline Voltage \#1: & $-0.000061 \mathrm{~V}$ \\
\hline Voltage \#2: & $0.000059 \mathrm{~V}$ \\
\hline Voltage \#3: & $-0.000035 \mathrm{~V}$ \\
\hline Voltage \#4: & $0.000033 \mathrm{~V}$ \\
\hline Voltage \#5: & $-0.000063 \mathrm{~V}$ \\
\hline Voltage \#6: & $0.000060 \mathrm{~V}$ \\
\hline Voltage \#7: & $-0.000034 \mathrm{~V}$ \\
\hline Voltage \#8: & $0.000035 \mathrm{~V}$ \\
\hline Voltage \#9: & $-0.000002 \mathrm{~V}$ \\
\hline Voltage \#10: & $0.000001 \mathrm{~V}$ \\
\hline Voltage \#11: & $-0.000052 \mathrm{~V}$ \\
\hline Voltage \#12: & $0.000055 \mathrm{~V}$ \\
\hline & $5 \times 10^{-3} \mathrm{~A}$ \\
\hline Current: & $0.0381 \mathrm{~cm}^{-12}$ \\
\hline Thickness: & $0.00160 \Omega-\mathrm{cm}^{-1}$ \\
\hline Resistivity: & $2164.46 \mathrm{~cm}^{2}-\mathrm{V}^{-1}$ \\
\hline Mobility: & $-1.81 \times 10^{18} \mathrm{~cm}^{-3}$ \\
\hline Density: &
\end{tabular}


Table 6.14: Hall results for GaAs at $5 \times 10^{-2} \mathrm{~A}$.

\begin{tabular}{|c|c|}
\hline \multicolumn{2}{|c|}{ GaAs } \\
\hline \multicolumn{2}{|c|}{} \\
\hline Voltage \#1: & $-0.000623 \mathrm{~V}$ \\
\hline Voltage \#2: & $0.000622 \mathrm{~V}$ \\
\hline Voltage \#3: & $-0.000356 \mathrm{~V}$ \\
\hline Voltage \#4: & $0.000357 \mathrm{~V}$ \\
\hline Voltage \#5: & $-0.000622 \mathrm{~V}$ \\
\hline Voltage \#6: & $0.000620 \mathrm{~V}$ \\
\hline Voltage \#7: & $-0.000355 \mathrm{~V}$ \\
\hline Voltage \#8: & $0.000358 \mathrm{~V}$ \\
\hline Voltage \#9: & $-0.000013 \mathrm{~V}$ \\
\hline Voltage \#10: & $0.000015 \mathrm{~V}$ \\
\hline Voltage \#11: & $-0.000543 \mathrm{~V}$ \\
\hline Voltage \#12: & $0.000544 \mathrm{~V}$ \\
\hline & $5 \times 10^{-2} \mathrm{~A}$ \\
\hline Current: & $0.0381 \mathrm{~cm}^{-2}$ \\
\hline Thickness: & $0.00165 \Omega-\mathrm{cm}^{-1}$ \\
\hline Resistivity: & $2149.81 \mathrm{~cm}^{2}-\mathrm{V}^{-1}-\mathrm{Sec}^{-1}$ \\
\hline Mobility: & $-1.76 \times 10^{18} \mathrm{~cm}^{-3}$ \\
\hline Density: & \\
\hline
\end{tabular}

Table 6.15: Hall results for GaAs at $1 \times 10^{-1} \mathrm{~A}$.

\begin{tabular}{|c|c|}
\hline \multicolumn{2}{|c|}{ GaAs } \\
\hline \multicolumn{2}{|c|}{} \\
\hline Voltage \#1: & $-0.001243 \mathrm{~V}$ \\
\hline Voltage \#2: & $0.001244 \mathrm{~V}$ \\
\hline Voltage \#3: & $-0.000711 \mathrm{~V}$ \\
\hline Voltage \#4: & $0.000716 \mathrm{~V}$ \\
\hline Voltage \#5: & $-0.001246 \mathrm{~V}$ \\
\hline Voltage \#6: & $0.001242 \mathrm{~V}$ \\
\hline Voltage \#7: & $-0.000712 \mathrm{~V}$ \\
\hline Voltage \#8: & $0.000714 \mathrm{~V}$ \\
\hline Voltage \#9: & $-0.000027 \mathrm{~V}$ \\
\hline Voltage \#10: & $0.000028 \mathrm{~V}$ \\
\hline Voltage \#11: & $-0.001088 \mathrm{~V}$ \\
\hline Voltage \#12: & $0.001088 \mathrm{~V}$ \\
\hline & $5 \times 10^{-2} \mathrm{~A}$ \\
\hline Current: & $0.0381 \mathrm{~cm}^{-12}$ \\
\hline Thickness: & $0.00165 \Omega_{-\mathrm{cm}}$ \\
\hline Resistivity: & $2151.35 \mathrm{~cm}^{2}-\mathrm{V}^{-1}-\mathrm{Sec}^{-1}$ \\
\hline Mobility: & $-1.76 \times 10^{18} \mathrm{~cm}^{-3}$ \\
\hline Density: &
\end{tabular}


Table 6.16: Average of the Hall resutls.

\begin{tabular}{|l|l|l|l|}
\hline Averages & GaN9906E & GaN9920 & GaAs \\
\hline Resistivity $[\Omega-\mathrm{cm}]$ & 10.838 & 35.815 & 0.00163 \\
\hline Mobility $\left[\mathrm{cm}^{2}-\mathrm{V}^{-1}-\mathrm{Sec}^{-1}\right]$ & 254.71 & 139.28 & 2158.36 \\
\hline Density $\left[\mathrm{cm}^{-3}\right]$ & $2.262 \times 10^{15}$ & $1.253 \times 10^{15}$ & $1.77 \times 10^{18}$ \\
\hline
\end{tabular}

